\title{
DOE/NV/10583--T2
}

\section{Ground-Water Data for 1990-91 and Ground-Water Withdrawals for 1951-91, Nevada Test Site and Vicinity, Nye County, Nevada}

By David B. Wood and Steven R. Reiner

U.S. GEOLOGICAL SURVEY

Open-File Report 96-475

Prepared in cooperation with the NEVADA OPERATIONS OFFICE, U.S. DEPARTMENT OF ENERGY, under Interagency Agreement DE-Al08-86NV10583

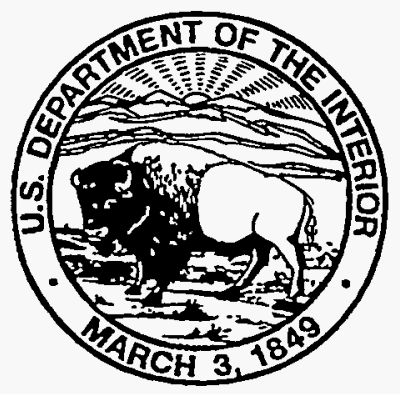

Carson City, Nevada 1996

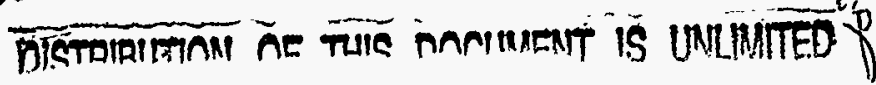

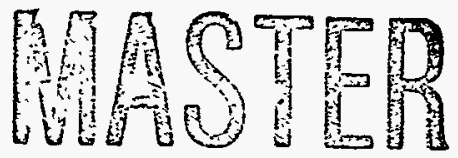




\title{
U.S. DEPARTMENT OF THE INTERIOR \\ BRUCE BABBITT, Secretary
}

\author{
U.S. GEOLOGICAL SURVEY \\ GORDON P. EATON, Director
}

Any use of trade names in this publication is for descriptive purposes

only and does not constitute endorsement by the U.S. Government

For additional information

write to:

District Chief

U.S. Geological Survey

333 West Nye Lane, Room 203

Carson City, NV 89706-0866
Copies of this report can be purchased from:

U.S. Geological Survey

Branch of Information Services

Box 25286

Denver, CO 80225-0046 


\section{DISCLAIMER}

Portions of this document may be illegible in electronic image products. Images are produced from the best available original document. 


\section{CONTENTS}

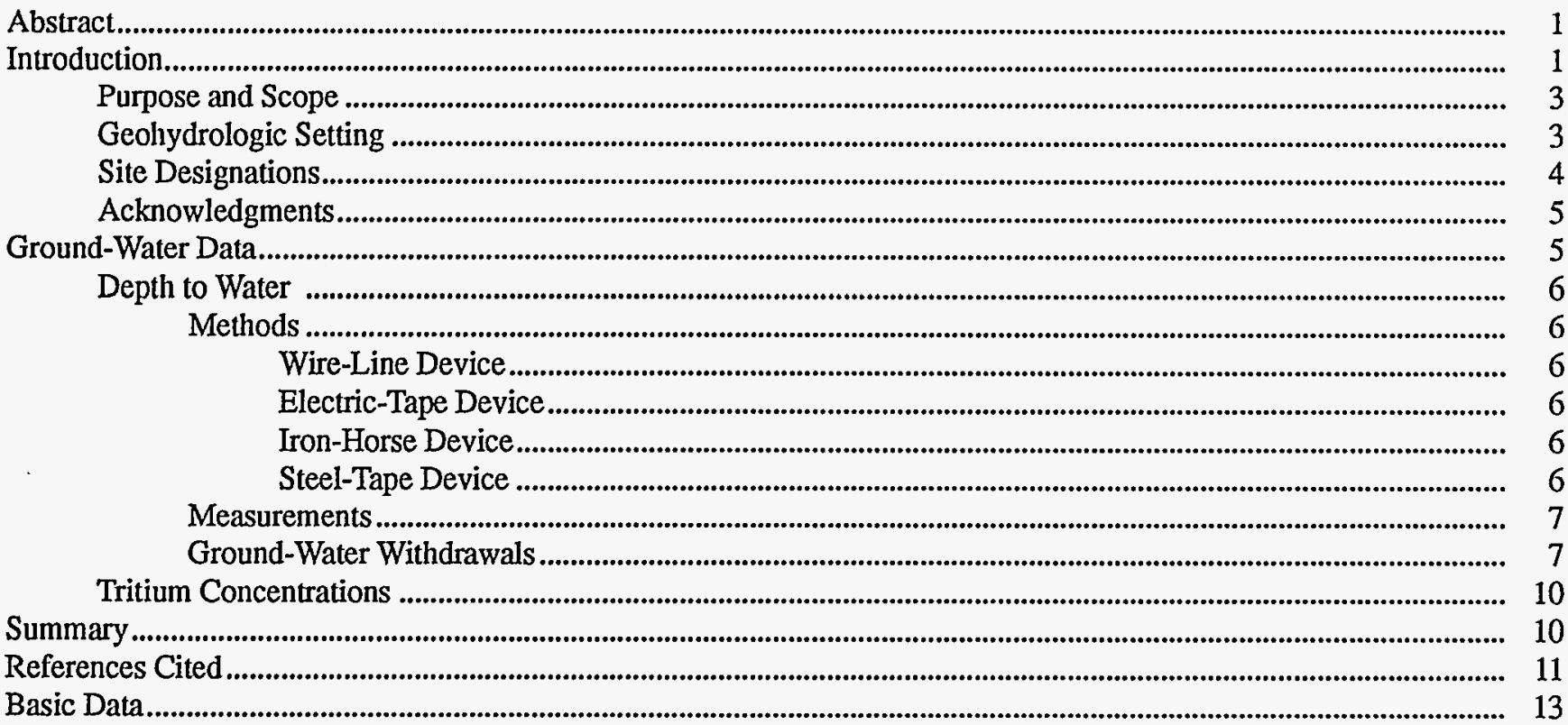

\section{PLATE}

[in pocket at back of report]

1. Map showing location of wells and test holes at the Nevada Test Site and vicinity, Nye County, Nevada

\section{FIGURES}

1. Map showing location of Nevada Test Site and vicinity, including Faultless Site....................................................... 2

2. Map showing location of test holes at Faultless Site, Nye County, Nevada .................................................................... 4

3. Graph showing annual ground-water withdrawals from the Nevada Test Site, 1951-91........................................... 14

4. Graph showing monthly ground-water withdrawals from the Nevada Test Site part of the

Alkali Flat-Furnace Creek Ranch ground-water subbasin, 1983-1991.

5. Graph showing monthly ground-water withdrawals from the Nevada Test Site part of the Ash Meadows ground-water subbasin, 1983-91

6-22. Graphs showing monthly and annual ground-water withdrawals from:

6. Well WW-8 on Buckboard Mesa

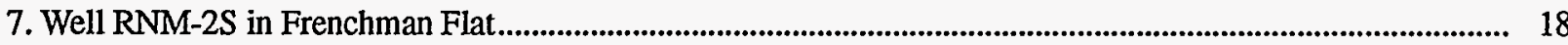

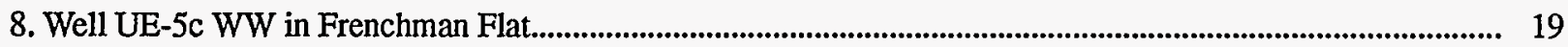

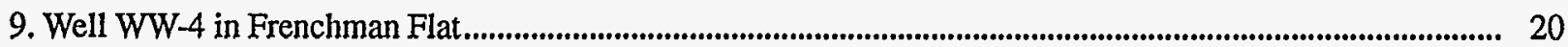

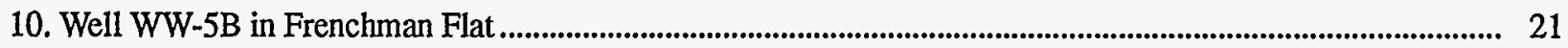

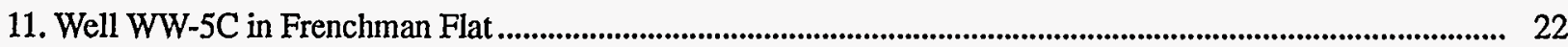

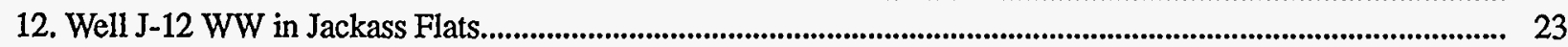

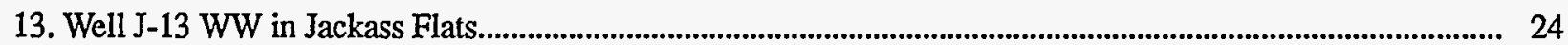

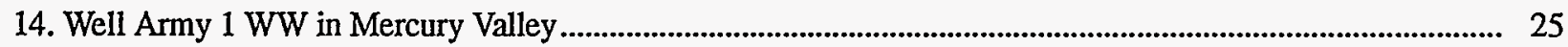

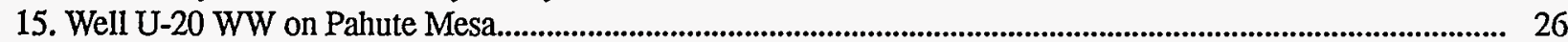

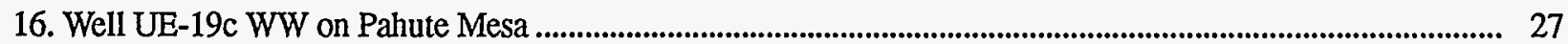

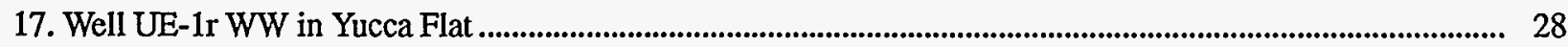

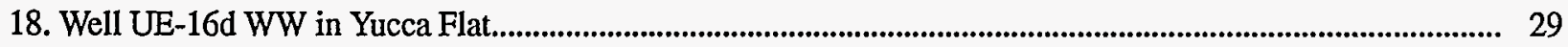

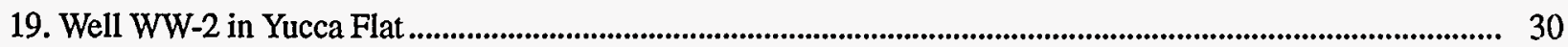

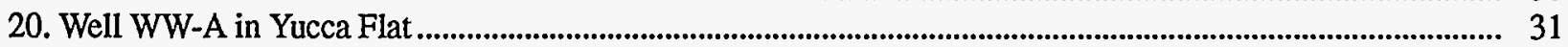

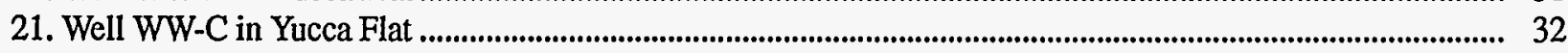

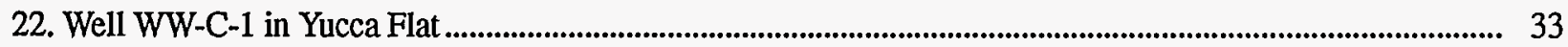




\section{TABLES}

1. Depth to water in wells and test holes at the Nevada Test Site and the Faultless Site, Nye County,

Nevada, water years 1990-91

2. Depth to water in ground-water supply wells at the Nevada Test Site, Nye County, Nevada, calendar years 1951-91

3. Annual withdrawals from ground-water supply wells at the Nevada Test Site, Nye County, Nevada, calendar years 1951-91

4. Tritium concentrations in water samples collected from test holes at the Nevada Test Site,

Nye County, Nevada, water years 1990-91.

\section{CONVERSION FACTORS AND VERTICAL DATUM}

\begin{tabular}{rcl}
\hline Multiply & By & To obtain \\
\hline acre-foot (acre-ft) & 1,233 & cubic meter \\
foot (ft) & 0.3048 & meter \\
gallon (gal) & 3.785 & liter \\
inch (in.) & 25.40 & millimeter \\
mile (mi) & 1.609 & kilometer \\
million gallons & 3.785 & million liters \\
picocurie (pCi) & 0.0370 & becquerel \\
square mile (mi $\left.{ }^{2}\right)$ & 2.590 & square kilometer \\
\hline
\end{tabular}

Sea level: In this report, "sea level" refers to the National Geodetic Vertical Datum of 1929 (NGVD of 1929, formerly called "Sea-Level Datum of 1929"), which is derived from a general adjustment of the first-order leveling networks of the United States and Canada.

Water year: Comprises the 12-month period from October 1 through September 30, and is designated by the year in which the period ends. 


\title{
Ground-Water Data for 1990-91 and Ground-Water Withdrawals for 1951-91, Nevada Test Site and Vicinity, Nye County, Nevada
}

\author{
By David B. Wood and Steven R. Reiner
}

\section{ABSTRACT}

The U.S. Geological Survey, in support of the U.S. Department of Energy Hydrology/Radionuclide Migration Program, collects and compiles hydrologic and geohydrologic data to aid in characterizing the regional and local ground-water flow systems underlying the Nevada Test Site. This report presents selected ground-water data collected from wells and test holes at and in the vicinity of the Nevada Test Site.

Depth-to-water measurements were made at 74 sites at and in the vicinity of the Nevada Test Site during water years 1990-91. Measured depths to water ranged from 301 to 2,215 feet below land surface and measured altitudes of the groundwater surface at the Nevada Test Site ranged from 2,091 to 6,083 feet above sea level. Depth-towater measurements were obtained by a combination of wire-line, electric-tape, iron-horse, and steel-tape methods.

Available annual ground-water withdrawals are reported for 24 water-supply wells for calendar years 1951-91. Available historic withdrawal and depth-to-water data for ground-water supply wells have been included to show changes through time. Total annual ground-water withdrawals from the 24 reported water-supply wells at the Nevada Test Site ranged from 33 to 1,118 million gallons, including 868 million gallons for 1990 and 713 million gallons for 1991. Annual ground-water withdrawals from individual water-supply wells ranged from 0 (non-use) to 325 million gallons; with 0 (non-use) to 233 million gallons for 1990 and 0 (non-use) to 155 million gallons for 1991.
Water samples were collected and analyzed for tritium concentrations at 15 sites during water years 1990-91. Tritium concentrations in bailed water samples ranged from below detection limits to $5,550,000$ picocuries per liter. Tritium concentrations in samples from three wells exceeded drinking-water standards established by the U.S. Environmental Protection Agency. All three wells are separate piezometers contained within a single test hole near an area of extensive underground nuclear testing.

\section{INTRODUCTION}

The Nevada Test Site (NTS; fig. 1) was established in 1950 as a continental proving ground for testing nuclear weapons (U.S. Congress, 1989). Atmospheric nuclear testing commenced in 1951 and underground nuclear testing commenced in 1957. Since 1962, all nuclear testing has been done underground (U.S. Department of Energy, 1988) and mostly in alluvium and volcanic rocks (U.S. Geological Survey, 1976). The United States entered into a unilateral moratorium on nuclear weapons testing on October 2, 1992 (U.S. Department of Energy, 1995). To date (1996), no nuclear tests have been made by the United States since September 1992.

The Faultless Site (fig. 1) was established to determine the suitability of using sites in central Nevada for underground nuclear tests of higher yield than was possible at NTS because of potential adverse seismic effects at Las Vegas, Nev. The Faultless nuclear test was detonated 3,199 ft below land surface in tuffaceous sediment and zeolitized tuff on January 19, 1968 (Thordarson, 1987). 


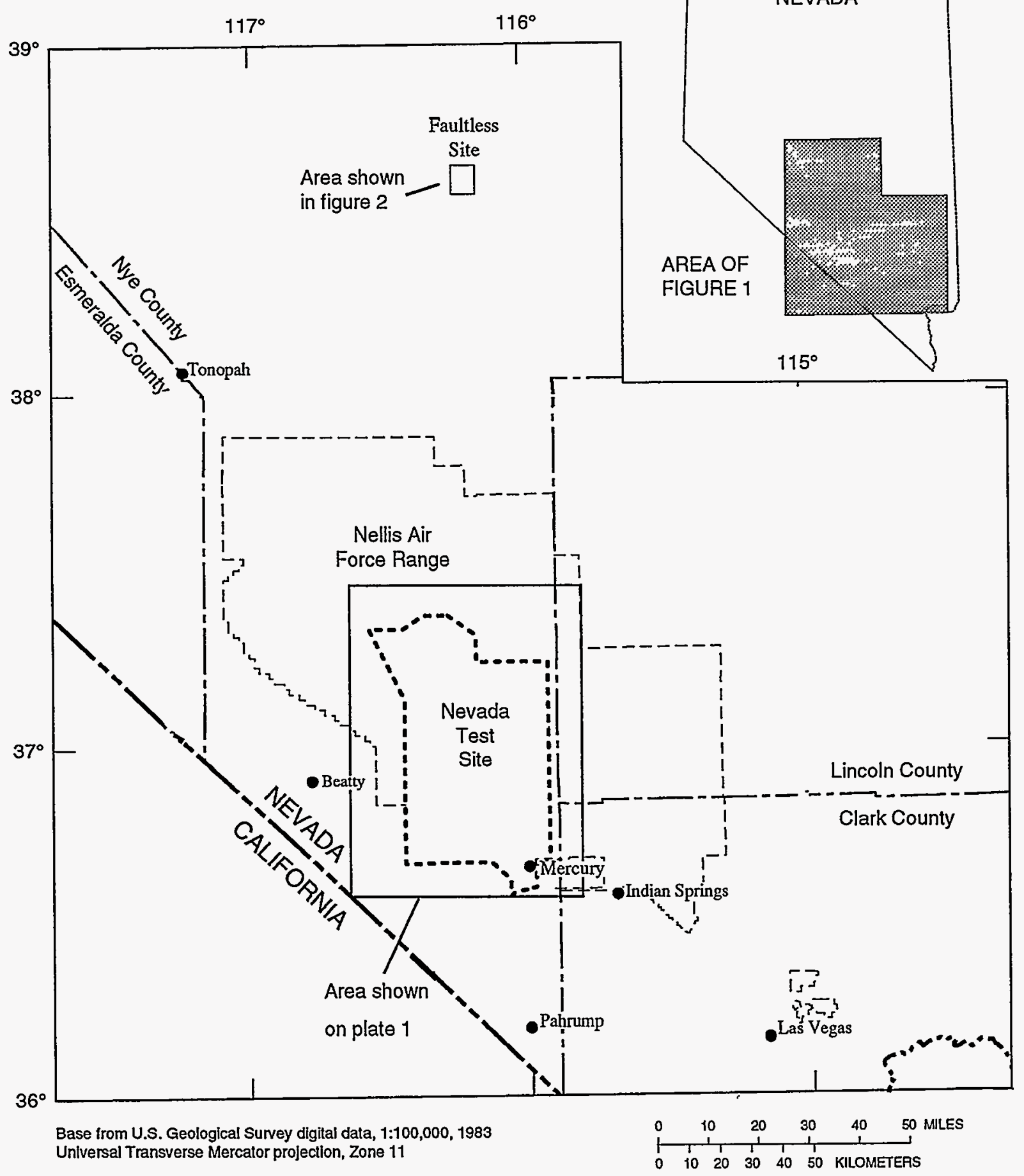

Figure 1. Location of Nevada Test Site and vicinity, including Faultless Site. 
The U.S. Geological Survey (USGS), Desert Research Institute, Los Alamos National Laboratory, and Lawrence Livermore National Laboratory are the principal organizations that provide services and technical expertise to the Nevada Operations Office of the U.S. Department of Energy in support of the Hydrology/Radionuclide Migration Program ${ }^{1}$ at NTS. The purpose of the Hydrology/Radionuclide Migration Program is to document the mechanisms by which radionuclides produced by underground nuclear tests may move through the geologic media, and the direction and extent of such movement. The specific responsibility of the USGS in support of the Hydrology/ Radionuclide Migration Program is to provide the necessary hydrologic and geohydrologic data, and the interpretation of these data, to aid in characterizing the regional and local ground-water flow systems. This characterization is needed to allow the U.S. Department of Energy to manage the ground-water resources at the Nevada Test Site and to assess the potential for radionuclide migration.

The U.S. Department of Energy's Yucca Mountain Site Characterization Project collects and compiles data in and adjacent to Areas 25 and 29 of NTS (pl. 1). Selected ground-water data collected for this project are compiled and reported by USGS personnel in the following reports: Robison (1984), Waddell and others (1984), Robison and others (1988), Gemmell (1990), McKinley and others (1991), O'Brien (1991), Luckey and others (1993), Boucher (1994), La Camera and Westenburg (1994), Lobmeyer and others (1995), and Tucci and others (1996).

The NTS, subdivided into 27 administrative areas (pl. 1), is about $70 \mathrm{mi}$ northwest of Las Vegas between latitudes 36 and 38 degrees north and longitudes 115 and 117 degrees west. The Faultless Site, in Hot Creek Valley (fig. 2), is about $70 \mathrm{mi}$ northeast of Tonopah between latitudes 38 and 39 degrees north and longitudes 116 and 117 degrees west. Both areas are in Nye County, Nev.

\section{Purpose and Scope}

This report presents ground-water data collected at and in the vicinity of NTS from October 1, 1989, to September 30, 1991 (water years 1990 and 1991).

${ }^{1}$ In 1992, the Hydrology/Radionuclide Migration Program was renamed the Hydrologic Resources Management Program.

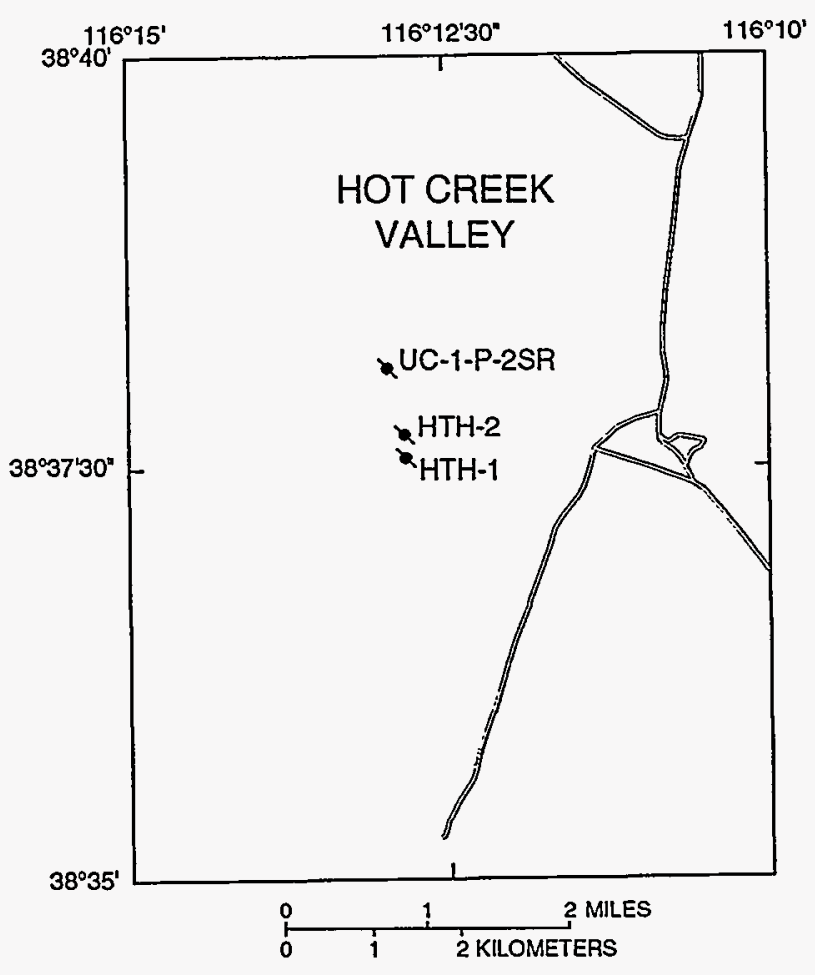

\section{EXPLANATION}

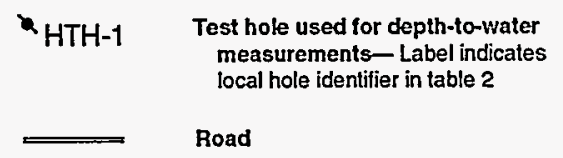

Figure 2. Location of test holes at Faultless Site, Nye County, Nevada.

Ground-water data presented in this report were collected from wells and test holes in two discrete study areas - the Nevada Test Site and the Faultless Site (fig. 1). Specifically, this report presents depth-to-water measurements collected from wells, test holes, and ground-water supply wells, withdrawals from groundwater supply wells, and tritium concentrations from wells and test holes. Available historical data on withdrawals and depth-to-water measurements (prior to October 1,1989 ) for ground-water supply wells have been included to show the amount of ground-water withdrawals and changes in depth to water through time.

\section{Geohydrologic Setting}

NTS is entirely within the Great Basin region of the Basin and Range physiographic province. The region is characterized by mountain ranges with a 
general north-south orientation separated by basins (valleys) that are filled by accumulations of unconsolidated to partly consolidated sedimentary deposits and underlain by consolidated rocks that also form the surrounding ranges (Stewart, 1980). The geohydrologic setting of NTS is similar to that of most of the Basin and Range province. It is characterized by localized aquifers within the basin-fill deposits and volcanic rocks. Regional aquifers are mostly within complexly folded and faulted limestones and dolomites that underlie the localized aquifers (Winograd and Thordarson, 1975), but also are within deep fractured volcanic rocks at some locations (Blankennagel and Weir, 1973). Much of the ground-water flow is interbasin; that is, deep flow is not controlled by the basin-andrange topography that defines surface-water drainage basins (Winograd and Thordarson, 1975).

NTS is contained within the Death Valley ground-water flow system (pl. 1), a regional system encompassing 15,800 $\mathrm{mi}^{2}$ (Harrill and others, 1988, sheet 1). Ground water flows from NTS to one of four major discharge areas: (1) Alkali Flat, (2) Ash Meadows, (3) Oasis Valley, and (4) Death Valley. All ground water beneath NTS is thought to flow within one of three ground-water subbasins of the Death Valley ground-water flow system-the Alkali Flat-Furnace Creek Ranch, Ash Meadows, and Oasis Valley subbasins (Waddell and others, 1984, p. 36-39 and pl. 3; Laczniak and others, 1996, p. 16-20 and pl. 1).

Ground water at NTS flows through three principal water-yielding units: (1) Cenozoic basin-fill deposits (primarily alluvium and colluvium of Quaternary and Tertiary age), (2) Cenozoic volcanic rock (primarily welded tuffs, thyolite lava flows, and basalt flows of Tertiary age), and (3) Paleozoic carbonate rock (primarily limestones and dolomites). Certain stratigraphic units and lithologic types (especially those that include fractured rocks) form the best water-yielding units as described by Winograd and Thordarson (1975, p. C1011), Blankennagel and Weir (1973, p. B5-6), Waddell and others (1984, p. 17-22), and Laczniak and others (1996, p. 10-16, 23-26, and 33-37).

\section{Site Designations}

Ground-water sites are either wells or test holes. The term "well" describes cased holes drilled specifically to find ground water. All other drilled holes are termed test holes. Wells and test holes are identified herein by Nevada Test Site (NTS) hole number, by U.S. Geological Survey (USGS) standard identification, and by the latitude and longitude of the site.

The NTS hole numbers were assigned by Raytheon Services Nevada to wells and test holes according to the type of hole drilled, site location (NTS Area, pl. 1), and sequence code for the consecutive order in which the hole was drilled or redrilled. Emplacement holes for nuclear weapons tests begin with the letter "U," followed by a dash (-), NTS area number (pl. 1), and sequence code (letters "a-z, aa-az, ba-bz, ..., za$\mathrm{zz}$ ). A hole drilled specifically to provide data that could not be collected from an emplacement hole is assigned incremental letters or numbers, or both. The suffix letters "ITS" indicate an integrated test system, "PS" a post-shot hole, "S" a substitute hole, and "WW" a water well. Exploratory holes follow the same naming convention, but begin with the letters "UE."

In this report, exceptions to the standard assignment procedures are Army $1 \mathrm{WW}, \mathrm{HTH}-1, \mathrm{HTH}-2$, J-11WW, J-12 WW, J-13 WW, PM-1, PM-2, PM-3, RNM-2S, TW-1, TW-3, TW-7, TW-B, TW-D, TW-F, UC-1-P-2SR, WW-2, WW-3, WW-4, WW-4A, WW5A, WW-5B, WW-5C, WW-8, WW-A, WW-C, and WW-C-1. The prefix letters "HTH" indicate a hydrologic test hole, "J" Jackass Flats, "PM" Pahute Mesa, "RNM" radionuclide migration, "TW" test well, "UC" underground central Nevada, and "WW" water well. Numbers and letters following the dash in the exception represent sequence of site drilling, not NTS location.

The USGS system for site identification is based on the latitude-longitude grid. Each site is identified by a unique 15-digit number: The first six digits are the degrees, minutes, and seconds of latitude; the next seven digits are the degrees, minutes, and seconds of longitude; and the last two digits constitute the sequence number of the well or test hole within the 1-second grid of latitude and longitude. The assigned number is retained as a permanent identifier even if a more precise latitude and longitude are later determined (U.S. Geological Survey, 1989, p. 2-10). Therefore, to determine the geographic location of a well or test hole, the latitude and longitude coordinates (which are listed herein) should be used rather than the USGS standard identification. 


\section{Acknowledgments}

Several organizations under contract with the Nevada Operations Office of the U.S. Department of Energy in 1991 made substantial contributions, including field inventory of wells and test holes and other hydrologic work. These contractors were Holmes and Narver, Inc.; Raytheon Services Nevada (formerly, Fenix and Scisson of Nevada); and Atlas Wireline Services (formerly, Dresser Atlas Industries, Inc.). Reynolds Electrical and Engineering Co., Inc. (REECo) provided ground-water withdrawal data for several wells and Los Alamos National Laboratory provided ground-water withdrawal data for well RNM2S. The Environmental Monitoring Systems Laboratory of the U.S. Environmental Protection Agency at Las Vegas analyzed water samples for tritium concentrations. On January 1, 1996, Bechtel Nevada replaced REECo as the prime contractor for the U.S. Department of Energy at NTS, and many of the organizations listed above are no longer involved at NTS.

\section{GROUND-WATER DATA}

Data presented consist of depth-to-water measurements taken from wells and test holes, groundwater withdrawals from ground-water supply wells, and tritium concentrations in water samples from wells and test holes. During water years 1990-91, depth to water was measured at 74 wells and test holes, groundwater withdrawals were compiled for 24 ground-water supply wells, and water samples were collected from 15 wells and test holes and analyzed for tritium concentration. The locations of these wells, test holes, and ground-water supply wells are shown on plate 1 and figure 2. Available historical data on withdrawals and depth-to-water measurements for ground-water supply wells have been included to show the amount of ground-water withdrawals and changes in depth to water through time. Depth-to-water data have been collected, compiled, verified, and stored in the GroundWater Site Inventory (GWSI) data base. This is one of four subsystems that constitute the computerized National Water Information System (NWIS) of the USGS, managed in Nevada by the District Office in Carson City, Nev.

The data-collection network consists of two parts-short-term test holes and long-term observation wells and test holes. Depth to water is measured intermittently in test holes that penetrate the saturated zone.
These measurements are made frequently until the measured depth to water stabilizes or the hole is destroyed or becomes inaccessible. Most test holes are short-term holes and the opportunity to measure depth to water in them is limited to a few weeks or a few months, which generally is not sufficient for stabilization of depth to water. Because most of the existing observation wells and test holes available for long-term observation were not drilled for the direct acquisition of hydrologic data, depth-to-water fluctuations that represent local (basin-fill or volcanic-rock) or regional (carbonate-rock or volcanic-rock) aquifer conditions generally are not monitored. Wells or test holes that exhibit minor fluctuations in depth to water are measured annually. Wells or test holes that exhibit large fluctuations in depth to water are measured weekly, quarterly, or continuously.

\section{Depth to Water}

The term "depth to water" is used rather than "water level" to avoid confusion with "static water level" as defined by Winograd (1970, p. 19) for NTS. Use of depth to water does not imply static conditions. Test holes drilled for nuclear-weapons tests commonly become inaccessible prior to fluid-level stabilization. Depth-to-water measurements in wells and test holes may represent elevated or depressed fluid levels because of disturbances caused by drilling or nearby nuclear detonations (Winograd, 1970, p. 20-25). Measurements may be affected by removal or injection of fluid or may represent a composite fluid level for the saturated units penetrated.

Water-level altitudes determined by subtracting depth-to-water measurements from the reported landsurface altitude may not represent actual water-level altitudes. The altitude of land surface is determined by conventional surveying techniques at each well or test hole when drilling operations have been completed. However, subsequent NTS activities may have altered the land-surface datum.

\section{Methods}

Several techniques are used to measure depth to water at NTS. Measurements in 1990-91 were made by the USGS with the wire-line, electric-tape, iron-horse, and steel-tape devices. Steel-tape measurements are used to calibrate the other devices. 


\section{Wire-Line Device}

The wire-line device consists of an armored fourconductor cable mounted on a motorized reel. The cable is centered over and guided into the well or test hole with a hydraulic boom. Attached to the end of the cable is one of two probes. One probe transmits an electric current to a meter at land surface by completing an electric circuit the instant water or other fluid is contacted. The other probe contains an enclosed float. Once this probe enters the water or fluid and the float moves far enough inside the probe to break the electric circuit, the meter reflects the change. A measuring wheel, over which the cable passes, measures the length of cable passed into and withdrawn from the well or test hole. The length of cable suspended below the reference mark is corrected for stretch and adjusted to land-surface datum to determine the measured depth to water.

\section{Electric-Tape Device}

The electric-tape device consists of a weighted electrical cable with two wire conductors exposed on the leading end. When both conductors contact water, electrical continuity between the two conductors is made and a visual display, sound beeper, or both is activated. The length of tape suspended below the reference mark is corrected for stretch and adjusted to landsurface datum to determine the measured depth to water.

\section{Iron-Horse Device}

The technique for using the iron-horse device to measure depth to water has been described by Garber and Koopman (1968, p. 6-11) and by Weir and Nelson (1976). The device consists of an armored single-conductor cable mounted on a portable reel. Attached to the end of the cable is a probe that transmits an electric current to a meter at land surface the instant water or fluid is contacted. A measuring wheel, over which the cable passes, measures the length of cable passed into and withdrawn from the well or test hole. The length of cable suspended below the reference mark is corrected for stretch and adjusted to land-surface datum to determine the measured depth to water.

\section{Steel-Tape Device}

The technique used for making steel-tape measurements of depth to water greater than $1,000 \mathrm{ft}$ below land surface described by Garber and Koopman (1968, p. 2-6) has been modified as described by Robison and others (1988, p. 9-10). The steel tape, which is mounted on a motor-driven reel, is suspended in the well or test hole a known distance below a reference mark so the bottom end is below the water or fluid surface. The length of wetted tape is subtracted from the suspended length below the reference mark and adjusted to landsurface datum by subtracting or adding the distance of the reference point above or below land surface to determine the measured depth to water.

The steel-tape device serves as a calibration tool to determine the stretch corrections applied to the wireline, electric-tape, and iron-horse devices. Periodically, simultaneous measurements are taken with each device from several wells and test holes chosen to represent a range of depth to water found at NTS. Corrections for the other devices are based on the measurements made with the steel-tape device.

\section{Measurements}

Depth-to-water measurements for wells and test holes shown in table 1 represent data collected during water years 1990-91. These measurements can be compared to earlier measurements in long-term observation wells and test holes reported by Wood (1992, p. 17-49) or later measurements reported by Reiner and others (1995, p. 21-28), Robie and others (1995, p. 21-32), Clary and others (1995, p. 641-652), and Bauer and others (1996, p. 670-702). Depth-to-water measurements for ground-water supply wells shown in table 2 represent available historic data. For wells and test holes listed in tables 1 and 2, the following data are presented: NTS hole number designation, USGS standard identification, latitude and longitude coordinates, date hole completed, land-surface altitude, hole dcpth, top of open interval, bottom of open interval, type of open interval, measurement date, depth-to-water measurcment, measurement method, and measurement site status. The wells and test holes are listed sequentially, first by NTS administrative area, then by NTS hole number designation and USGS standard identification within each area.

Some items listed in tables 1 and 2 need further clarification. "Date hole completed" is the date that borehole construction work, which may affect where water enters the casing or borehole, ceased. Types of construction work that affect completion are borehole drilling, setting or perforating casing, setting packers or plugs, and grouting the hole. Therefore, completion 
dates listed in table 1 and 2 may not agree with completion dates reported by Raytheon Services Nevada (RSN). "Hole depth" is the accessible hole depth, which may be shallower than total drilled depth. Because hole depth represents the most recent available information, the present accessible hole depth may be shallower or deeper than the hole depth reported by RSN. "Depth of open intervals," top and bottom, are listed for each perforated or packer interval or borehole diameter. The bottom of the deepest open interval normally is equivalent to total drilled depth. Water may be contributed to the casing or borehole from depths greater than the accessible depth if drilled depth exceeds accessible depth. A site status of " $Z$ " is normally listed if the first depth-to-water measurement was taken within about 1 month of the date that the hole was completed to indicate that the water level may not have stabilized within the casing or borehole. A site status of " $Z$ " also denotes nearby nuclear events, earthquakes, or other natural or man-induced conditions that may affect the measured depth to water.

The measured depth to water ranged from $301 \mathrm{ft}$ below land surface on September 12, 1991, at UE-4t 1 in Yucca Flat to 2,215 ft below land surface on September 25, 1990, at U-20be on Pahute Mesa. The measured altitude of the ground-water surface ranged from 2,091 $\mathrm{ft}$ above sea level on January 24, 1990, at UE-3e 4 in Yucca Flat to $6,083 \mathrm{ft}$ above sea level on October 12 , 1989, at UE-12t 6 on Rainier Mesa.

\section{Ground-Water Withdrawals}

Ground-water withdrawals are compiled for 24 wells at NTS. Withdrawal data included for the 195171 calendar years are reprinted from Claassen (1973), except for J-11 WW. Records of total ground-water withdrawals for 1972-82 are not available except for J-12 WW and RNM-2S. Withdrawal data for well RNM-2S for 1975-91 were supplied by Los Alamos National Laboratory (J.L. Thompson, written commun., 1992). All other withdrawal data for the 1983-91 calendar years were compiled from water-production reports provided by REECo.

All water-supply wells currently in use at NTS are equipped with totalizing flow meters. Each meter has a counting-wheel display (similar to an automobile odometer) with six rolling digits and either two or three fixed digits. Meters with two fixed digits are accurate to about the nearest 100 gallons and meters with three fixed digits are accurate to about the nearest 1,000 gal- lons. REECo personnel recorded meter readings periodically and the totals represent the difference in readings from one date to the next. REECo waterproduction data have been recompiled to represent average monthly and yearly totals. Withdrawals not reported due to broken flow meters were estimated by taking an average of the REECo data on ground-water withdrawals prior to and following the missing period of record. When a meter was broken for a lengthy period, trends from preceding years were considered.

Ground-water withdrawals from water-supply wells at NTS are presented in table 3. The following information is listed: NTS hole-number designation, USGS standard identification, latitude and longitude coordinates, date well completed, land-surface altitude, well depth, top of open interval, bottom of open interval, type of open interval, primary water-yielding units, ground-water subbasin, calendar year of ground-water withdrawals, ground-water withdrawals in million gallons, ground-water withdrawals in acre-feet, source of withdrawal data, and days of estimated withdrawals. Data are listed in table 3 sequentially, first by NTS administrative area, by NTS hole-number designation, then by USGS site identification number.

Total annual ground-water withdrawals at NTS ranged from about 33 million gallons (pumped from 3 water-supply wells for 1951) to about 1,118 million gallons (pumped from 14 water-supply wells for 1989). Annual ground-water withdrawals from individual water-supply wells ranged from 0 (non-use) to $325 \mathrm{mil}$ lion gallons (pumped from RNM-2S in Frenchman Flat for 1979). The total ground-water withdrawal at NTS for 1990 was 868 million gallons (pumped from 13 water-supply wells) and for 1991 was 713 million gallons (pumped from 13 water-supply wells). The maximum ground-water withdrawal from a water-supply well for 1990 was 233 million gallons and for 1991 was 155 million gallons (both pumped from RNM-2S).

Total annual ground-water withdrawals from NTS for calendar years 1951-71 and 1983-91 are shown in figure 3 . No records were available for $1972-$ 74. Only withdrawals from well RNM-2S are included for 1975-80. Only withdrawals from wells RNM-2S and J-12 WW are included for 1981-82. Water-production records of withdrawals from other active watersupply wells for 1972-82 are not available. Total monthly withdrawals for the 1983-91 calendar years from NTS part of the Alkali Flat-Furnace Creek Ranch ground-water subbasin, from the volcanic rock wateryielding unit, are shown in figure 4 . Total monthly 
withdrawals for calendar years 1983-91 from the NTS part of the Ash Meadows ground-water subbasin, by water-yielding unit, are shown in figure 5. No withdrawals were made for the 1983-91 calendar years from the NTS part of the Oasis Valley ground-water subbasin. Where more than one water-yielding unit was saturated, units were combined. No attempt was made to determine percent contribution by unit. If a unit was saturated and open to the well bore, it was reported.

Monthly and annual ground-water withdrawals, by water-supply well for calendar years 1983-91, are shown in figures 6-22. The graphs are grouped by geographic area and, within an area, by NTS hole-number designation. Data are compiled from flow-meter records except where noted. Consistently throughout the REECo water-production data, withdrawals were estimated for all active wells for 8 days in December 1984, 13 days for January 1985, and 7 days for December 1985. A brief description of each graph follows.

Ground-water withdrawals from well WW-8 on Buckboard Mesa (Area 18, pl. 1) are shown in figure 6. The well is in the Alkali Flat-Furnace Creek Ranch ground-water subbasin, open to Tertiary volcanic rock, and used primarily for drinking water and construction. Data are compiled from flow-meter records except as noted above, 5 days estimated for June 1989, and 23 days for July 1989.

Ground-water withdrawals from well RNM-2S in Frenchman Flat (Area 5, pl.1) are shown in figure 7. The well is in the Ash Meadows ground-water subbasin, open to Quaternary alluvium, and used for a radionuclide migration experiment. Data are compiled from flow-meter records. Pumping ceased when the experiment was completed August 29, 1991.

Ground-water withdrawals from well UE-5c WW in Frenchman Flat (Area 5, pl.1) are shown in figure 8. The well is in the Ash Meadows ground-water subbasin, open to Quaternary alluvium and Tertiary volcanic rock, and used primarily for drinking water and construction. Data are compiled from flow-meter records as noted above. The well was not pumped during April 1983, November 1986 through March 1987, May through July 1987, and April 1989 through February 1991.

Ground-water withdrawals from well WW-4 in Frenchman Flat (Area 6, pl. 1) are shown in figure 9. The well is in the Ash Meadows ground-water subbasin, open to Tertiary volcanic rock, and used primarily for drinking water and construction. Data are compiled from flow-meter records except as noted above, 19 days estimated for August 1990, and 9 days for September 1990 . The well was not pumped January through March 1983.

Ground-water withdrawals from well WW-5B in Frenchman Flat (Area 6, pl. 1) are shown in figure 10. The well is in the Ash Meadows ground-water subbasin, open to Quaternary alluvium, and used primarily for drinking water and construction. Data are compiled from flow-meter records except as noted above. Pumping ceased October 1988.

Ground-water withdrawals from well WW-5C in Frenchman Flat (Area 5, pl. 1) are shown in figure 11. The well is in the Ash Meadows ground-water subbasin, open to Quaternary alluvium, and used primarily for drinking water and construction. Data are compiled from flow-meter records as noted above. The well was not pumped October through December 1985.

Ground-water withdrawals from well J-12 WW in Jackass Flats (Area 25, pl. 1) are shown in figure 12. The well is in the Alkali Flat-Furnace Creek Ranch ground-water subbasin, open to Tertiary volcanic rock, and used primarily for drinking water and construction. Data.are compiled from flow-meter records except as noted above.

Ground-water withdrawals from well J-13 WW in Jackass Flats (Area 25, pl. 1) are shown in figure 13. The well is in the Alkali Flat-Furnace Creek Ranch ground-water subbasin, open to Tertiary volcanic rock, and used primarily for drinking water and construction. Data are compiled from flow-meter records except as noted above, 11 days estimated for November 1989, 3 days for December 1989, and 7 days for September 1990.

Ground-water withdrawals from well Army 1 WW in Mercury Valley (Area 22, pl. 1) are shown in figure 14. The well is in the Ash Meadows groundwater subbasin, open to Paleozoic carbonate rock, and used primarily for drinking water and construction. Data are compiled from flow-meter records except as noted above, 7 days estimated for June 1987, 14 days for November 1988, 8 days for December 1990, and 31 days for January 1991.

Ground-water withdrawals from well U-20 WW on Pahute Mesa (Area 20, pl. 1) are shown in figure 15. The well is in the Alkali Flat-Furnace Creek Ranch ground-water subbasin, open to Tertiary volcanic rock, and used primarily for construction. The well was completed in July 1985. Data are compiled from flow-meter 
records except 7 days estimated for December 1985 and 7 days for August 1986. The well was not pumped June through September 1991.

Ground-water withdrawals from well UE-19c WW on Pahute Mesa (Area 19, pl. 1) are shown in figure 16. The well is in the Alkali Flat-Furnace Creek Ranch ground-water subbasin, open to Tertiary volcanic rock, and used primarily for construction. Data are compiled from flow-meter records except as noted above. The well was not pumped May 1983, January through March 1984, and January 1988.

Ground-water withdrawals from well UE-1rWW in Yucca Flat (Area 1, pl. 1) are shown in figure 29. The well is in the Ash Meadows ground-water subbasin, open to Tertiary volcanic rock and Paleozoic carbonate rock, and used primarily for construction. Data are compiled from flow-meter records except as noted above. Pumping ceased October 1988.

Ground-water withdrawals from well UE-16d WW in Yucca Flat (Area 16, pl. 1) are shown in figure 18. The well is in the Ash Meadows ground-water subbasin, open to Paleozoic carbonate rock, and used primarily for drinking water. Data are compiled from flow-meter records except as noted above.

Ground-water withdrawals from well WW-2 in Yucca Flat (Area 2, pl. 1) are shown in figure 19. The well is in the Ash Meadows ground-water subbasin, open to Paleozoic carbonate rock, and used primarily for construction. Data are compiled from flow-meter records except as noted above, 5 days estimated for June 1989, and 23 days for July 1989. The well was not pumped during May 1989. Pumping ceased December 1990.

Ground-water withdrawals from well WW-A in Yucca Flat (Area 3, pl. 1) are shown in figure 20. The well is in the Ash Meadows ground-water subbasin, open to Quatemary alluvium, and used primarily for drinking water. Data are compiled from flow-meter records except as noted above. Pumping ceased October 1988 .

Ground-water withdrawals from well WW-C in Yucca Flat (Area 6, pl. 1) are shown in figure 21. The well is in the Ash Meadows ground-water subbasin, open to Paleozoic carbonate rock, and used primarily for drinking water. Data are compiled from flow-meter records except as noted above, 7 days estimated for September 1986, and 7 days for September 1990. The well was not pumped December 1987, March through May 1989, and December 1989 through January 1990.
Ground-water withdrawals from well WW-C-1 in Yucca Flat (Area 6, pl. 1) are shown in figure 22. The well is in the Ash Meadows ground-water subbasin, open to Paleozoic carbonate rock, and used primarily for drinking water. Data are compiled from flow-meter records except as noted above and 7 days estimated for September 1988.

Graphs of ground-water withdrawals are not included for the following wells because the wells are no longer used for water supply or current records are not available. Wells WW-5A and WW-3 have not been used for water supply since 1970 , wells UE-19e WW, UE-19gS WW, and U-20a 2 WW have not been used since 1967, and well J-11WW has not been used since 1960. Water from well UE-15d WW was used intermittently for cleaning purposes at the U.S. Environmental Protection Agency farm until the pump failed to start and pumping ceased October 7, 1991. Well WW-4A was drilled in 1990 and was not used.

\section{Tritium Concentrations}

Water samples for determination of tritium concentrations were collected from 15 wells and test holes at NTS. The technique used to collect the samples from slightly below the water surface is described by Claassen (1982, p. 36-38) and by Wood (1976, p. 5-6). The bailer used in 1990 and 1991 was a solid plug coupled to a $1-9 / 16$-in. (inside diameter) by 6 -ft stainless steel tube coupled to a 2 -ft upper section containing four $3 / 4^{-}$ in. by 6 -in. slots and coupled to a solid cap. Each bailed sample contained about $1 / 2$ gallon of water. Raw, unfiltered samples were collected in 500-milliliter acidrinsed glass bottles, labeled, and delivered to the Environmental Monitoring Systems Laboratory of the U.S. Environmental Protection Agency in Las Vegas, Nev. To prevent possible cross-contamination after sampling, the bailer was rinsed twice with a 10 percent hydrochloric acid solution. The bailer then was rinsed twice with tap water or deionized water and allowed to air dry prior to the next sample collection.

The results of the tritium analyses are presented in table 4. For the 15 wells and test holes, the following information is listed: NTS hole-number designation, USGS standard identification, latitude and longitude coordinates, date hole completed, land-surface altitude, hole depth, top of open interval, bottom of open interval, type of open interval, sample date, and tritium concentration. Data are listed in table 4 sequentially, first by NTS administrative area, by NTS hole-number des- 
ignation, then by USGS site identification number. Because some of the wells and test holes may not have been completely developed (pumped) prior to sample collection, some tritium concentrations may represent residual drilling fluids or other fluids introduced during drilling or pumping and water injection instead of representing water solely from the saturated zone.

Tritium concentrations ranged from below detection limits $(-1 \mathrm{pCi} / \mathrm{L})$ on September 10,1991 , at U-20ax on Pahute Mesa to $5,550,000 \mathrm{pCi} / \mathrm{L}$ on May 3, 1990, at UE-3e 4-1 in Yucca Flat. An average annual concentration of $20,000 \mathrm{pCi} / \mathrm{L}$ of tritium in drinking water is the maximum permissible limit established by the U.S. Environmental Protection Agency in Title 40, Code of Federal Regulations (1988). Tritium concentrations in samples from wells UE-3e 4-1, UE-3e 4-2, and UE-3e 4-3 exceeded this limit. These three wells are contained within a single test hole near an area of extensive underground nuclear testing. Each well is a separate piezometer open to a different depth interval.

\section{SUMMARY}

The U.S. Geological Survey, in support of the U.S. Department of Energy Hydrology/Radionuclide Migration Program, collects and compiles hydrologic and geohydrologic data at and in the vicinity of NTS to aid in characterizing the regional and local groundwater flow systems. This report presents depth-towater measurements, ground-water withdrawals, and tritium concentrations determined for selected wells and test holes as part of this program.

Depth to water was measured at 74 wells and test holes at and in the vicinity of NTS during October 1 , 1989, to September 30, 1991. The depth to water ranged from $301 \mathrm{ft}$ below land surface at UE-4t 1 to $2,215 \mathrm{ft}$ below land surface at U-20be. The measured altitude of the water surface ranged from $2,091 \mathrm{ft}$ above sea level at UE-3e 4 to $6,083 \mathrm{ft}$ above sea level at UE12t 6 . Available historic depth-to-water measurement data collected from water-supply wells are reported also. Wire-line, electric tape, iron-horse, and steel-tape devices were used to measure depth to water.

Available annual ground-water withdrawals were reported for 24 water-supply wells for calendar years 1951-91. Total annual ground-water withdrawals from the 24 reported water-supply wells at NTS ranged from 33 million gallons for 1951 to 1,118 million gallons for 1989. Annual ground-water withdrawals from individ- ual water-supply wells ranged from 0 (non-use), for several wells during one or more years, to 325 million gallons, from RNM-2S for 1979. The total groundwater withdrawal for 1990 was 868 million gallons and for 1991 was 713 million gallons. The maximum withdrawal from a water-supply well for 1990 was 233 million gallons and for 1991 was 155 million gallons, both from RNM-2S.

Tritium concentrations in water samples collected from selected wells and test holes penetrating the saturated zone ranged from below detection limits at $\mathrm{U}-20 \mathrm{ax}$ to $5,550,000 \mathrm{pCi} / \mathrm{L}$ at $\mathrm{UE}-3 \mathrm{e}$ 4-1. Tritium concentrations in samples from wells UE-3e 4-1, UE-3e 4-2, and UE-3e 4-3 exceeded established drinking-water standards. All three wells are separate piezometers contained within a single test hole near an area of extensive underground nuclear testing.

\section{REFERENCES CITED}

Bauer, D.J., Foster, B.J., Joyner, J.D., and Swanson, R.A., 1996, Water resources data, Nevada, water year 1995: U.S. Geological Survey Water Data Report NV-95-1, $734 \mathrm{p}$.

Blankennagel, R.K., and Weir, J.E., Jr., 1973, Geohydrology of the eastern part of Pahute Mesa, Nevada Test Site, Nye County, Nevada: U.S. Geological Survey Professional Paper 712-B, 35 p.

Boucher, M.S., 1994, Water levels in wells J-11 and J-12, 1989-91, Yucca Mountain area, Nevada: U.S. Geological Survey Open-File Report 94-303, 9 p.

Claassen, H.C., 1973, Water quality and physical characteristics of Nevada Test Site water-supply wells: U.S. Geological Survey Open-File Report USGS-474-158, $145 \mathrm{p}$.

1982, Guidelines and techniques for obtaining water samples that accurately represent the water chemistry of an aquifer: U.S. Geological Survey Open-File Report 82-1024, $49 \mathrm{p}$.

Clary, S.L., McClary, D.R., Whitney, Rita, and Reeves, D.D., 1994, Water resources data, Nevada, water year 1994: U.S. Geological Survey Water Data Report NV-94-1, $768 \mathrm{p}$.

Code of Federal Regulations, 1988, Title 40, Section - 141, Drinking Water Regulations: Federal Register, Volume 41.

Garber, M.S., and Koopman, F.C., 1968, Methods of measuring water levels in deep wells: U.S. Geological Survey Techniques of Water-Resources Investigations, Book 8, Chapter A1, 23 p. 
Gemmell, J.M., 1990, Water levels in periodically measured wells in the Yucca Mountain area, Nevada, 1988: U.S. Geological Survey Open-File Report 90-113, 47 p.

Harrill, J.R., Gates, J.S., and Thomas, J.M., 1988, Major ground-water flow systems in the Great Basin region of Nevada, Utah, and adjacent states: U.S. Geological Survey Hydrologic Investigations Atlas HA-694-C, 2 sheets.

La Camera, R.J., and Westenburg, C.L., 1994, Selected ground-water data for Yucca Mountain region, southern Nevada and eastern California, through December 1992: U.S. Geological Survey Open-File Report 94-54, $161 \mathrm{p}$.

Laczniak, R.J., Cole, J.C., Sawyer, D.A., and Trudeau, D.A., 1996, Summary of hydrogeologic controls on groundwater flow at the Nevada Test Site, Nye County, Nevada: U.S. Geological Survey Water-Resources Investigations Report 96-4109, 59 p.

Lobmeyer, D.H., Luckey, R.R., O'Brien, G.M., and Burkhardt, D.J., 1995, Water levels in continuously monitored wells in the Yucca Mountain area, Nevada, 1989: U.S. Geological Survey Open-File Report 93-98, $173 \mathrm{p}$.

Luckey, R.R., Lobmeyer, D.H., and Burkhardt, D.J., 1993, Water levels in continuously monitored wells in the Yucca Mountain area, Nevada, 1985-88: U.S. Geological Survey Open-File Report 91-493, 252 p.

McKinley, P.W., Long, M.P., and Benson, L.V., 1991, Chemical analyses of water from selected wells and springs in the Yucca Mountain area, Nevada and southeastern California: U.S. Geological Survey Open-File Report 90-355, 47 p.

O'Brien, G.M., 1991, Water levels in periodically measured wells in the Yucca Mountain area, Nevada, 1989: U.S. Geological Survey Open-File Report 91-178, 51 p.

Reiner, S.R., Locke, G.L., and Robie, L.S., 1995, Groundwater data for the Nevada Test Site and selected other areas in south-central Nevada, 1992-93: U.S. Geological Survey Open-File Report 95-160, 38 p.

Robie, L.S., Reiner, S.R., and Locke, G.L., 1995, Groundwater data for the Nevada Test Site, 1992, and for selected other areas in south-central Nevada, 1952-92: U.S. Geological Survey Open-File Report 95-284, 48 p.

Robison, J.H., 1984, Ground-water level data and preliminary potentiometric surface maps, Yucca Mountain and vicinity, Nye County, Nevada: U.S. Geological Survey Water-Resources Investigations Report 84-4197, 8 p.

Robison, J.H., Stephens, D.M., Luckey, R.R., and Baldwin, D.A., 1988, Water levels in periodically measured wells in the Yucca Mountain area, Nevada, 1981-87: U.S. Geological Survey Open-File Report 88-468, 132 p.
Stewart, J.H., 1980, Geology of Nevada-A discussion to accompany the geologic map of Nevada: Nevada Bureau of Mines and Geology Special Publication 4, 136

Thordarson, William, 1987, Hydrogeology of the Faultless Site, Nye County, Nevada: U.S. Geological Survey Water-Resources Investigations Report 86-4342, 40 p.

Tucci, Patrick, O'Brien, G.M., and Burkhardt, D.J., 1996, Water levels in the Yucca Mountain area, Nevada, 1990-91: U.S. Geological Survey Open-File Report 94$111,107 \mathrm{p}$.

U.S. Congress, 1989, The containment of underground nuclear explosions: Office of Technology Assessment Report OTA-ISC-414, $80 \mathrm{p}$.

U.S. Department of Energy, 1988, Announced United States nuclear tests, July 1945 through December 1987: Nevada Operations Office, Office of Public Affairs Report NVO-209, Revision 8, 66 p. 1995, United States nuclear tests, July 1945 through September 1992: Nevada Operations Office, Office of External Affairs Report NVO-209, Revision 14, 97 p.

U.S. Geological Survey, 1976, Field trip to Nevada Test Site: U.S. Geological Survey Open-File Report 76-313, 64 p. 1989, National Water Information System user's manual, volume 2, chapter 4. Ground-Water Site Inventory System: U.S. Geological Survey Open-File Report $89-587,283 \mathrm{p}$.

Waddell, R.K., Robison, J.H., and Blankennagel, R.K., 1984, Hydrology of Yucca Mountain and vicinity, Nevada-California-Investigative results through mid1983: U.S. Geological Survey Water-Resources Investigations Report 84-4267, 72 p.

Weir, J.E., Jr., and Nelson, J.W., 1976, Operation and maintenance of a deep-well water-level measurement device, the "iron horse": U.S. Geological Survey WaterResources Investigations 76-27, $28 \mathrm{p}$.

Winograd, I.J., 1970, Noninstrumental factors affecting measurement of static water levels in deeply buried aquifers and aquitards, Nevada Test Site: Ground Water, v. 8, no. 2, p. 19-28.

Winograd, I.J., and Thordarson, William, 1975, Hydrogeologic and hydrochemical framework, south-central Great Basin, Nevada-California, with special reference to the Nevada Test Site: U.S. Geological Survey Professional Paper 712-C, $126 \mathrm{p}$.

Wood, D.B., 1992, Ground-water data collected at the Nevada Test Site and vicinity, Nye County, Nevada, water years 1988-89: U.S. Geological Survey OpenFile Report 92-130, $50 \mathrm{p}$.

Wood, W.W., 1976, Guidelines for collection and field analysis of ground-water samples for selected unstable constituents: U.S. Geological Survey Techniques of WaterResources Investigations, Book 1, Chapter D2, 24 p. 


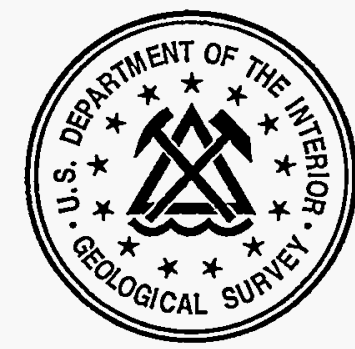




\section{BASIC DATA}




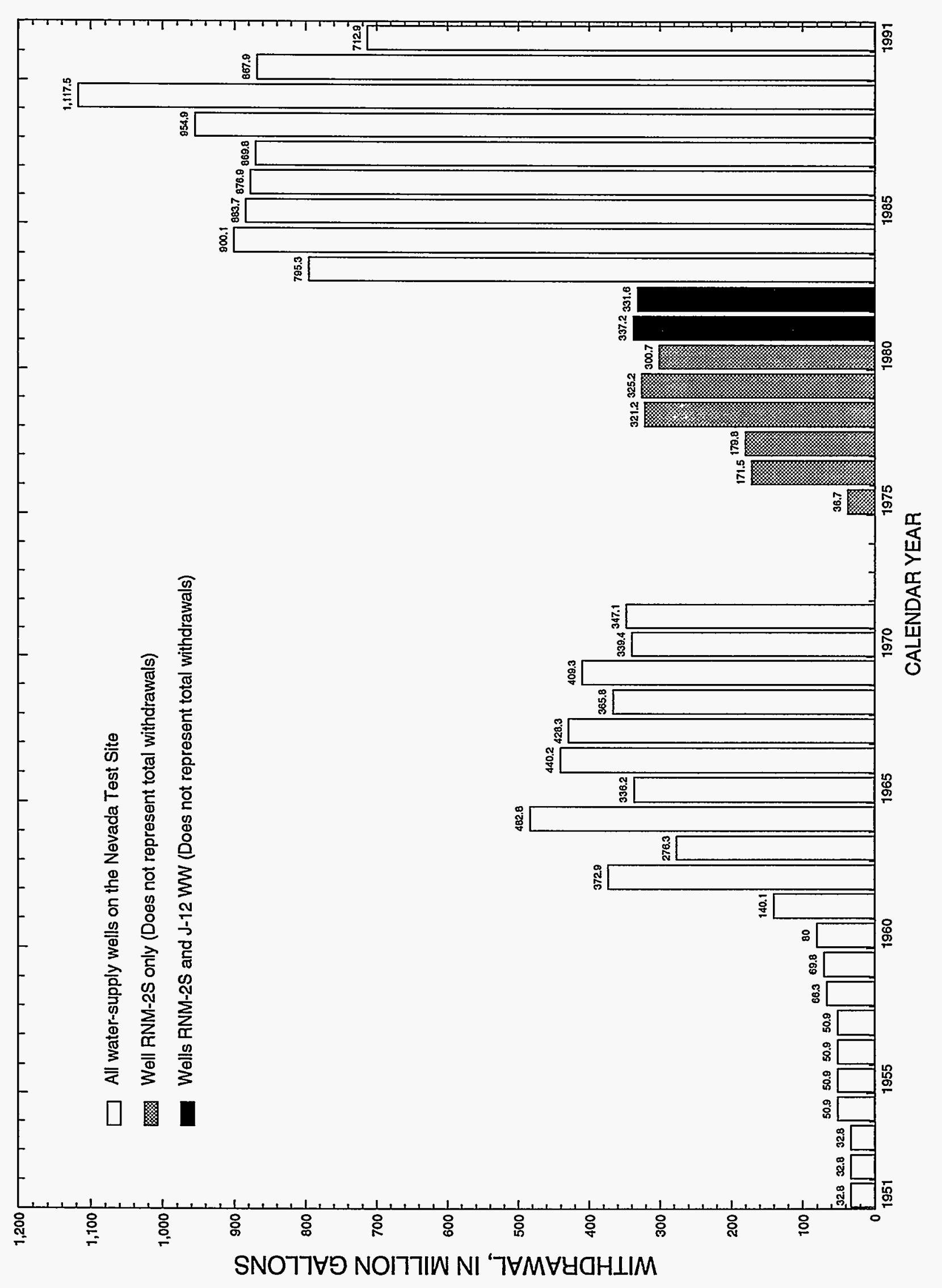




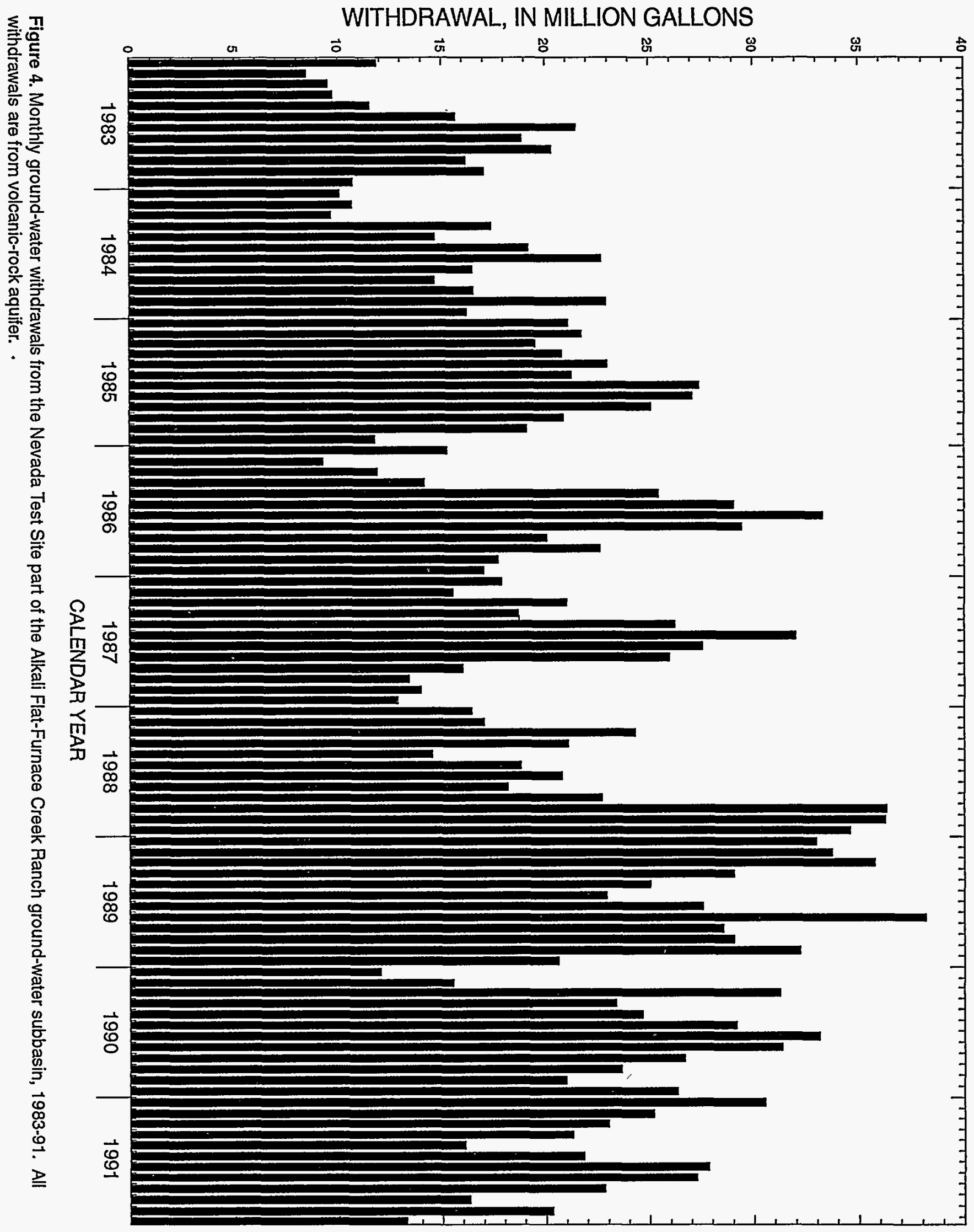




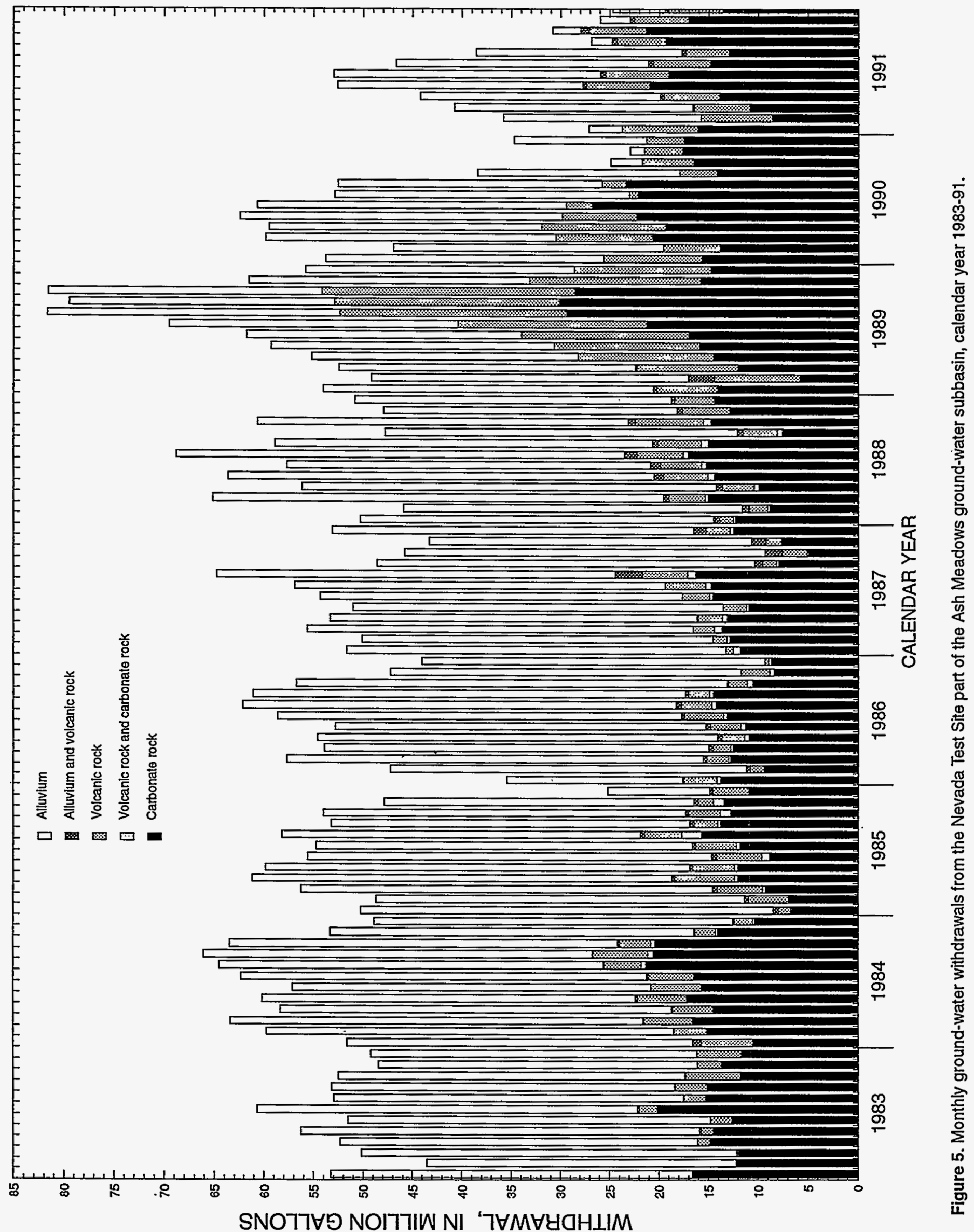

16 Ground-Water Data for 1990-91 and Ground-Water Withdrawals for 1951-91, Nevada Test SIte 


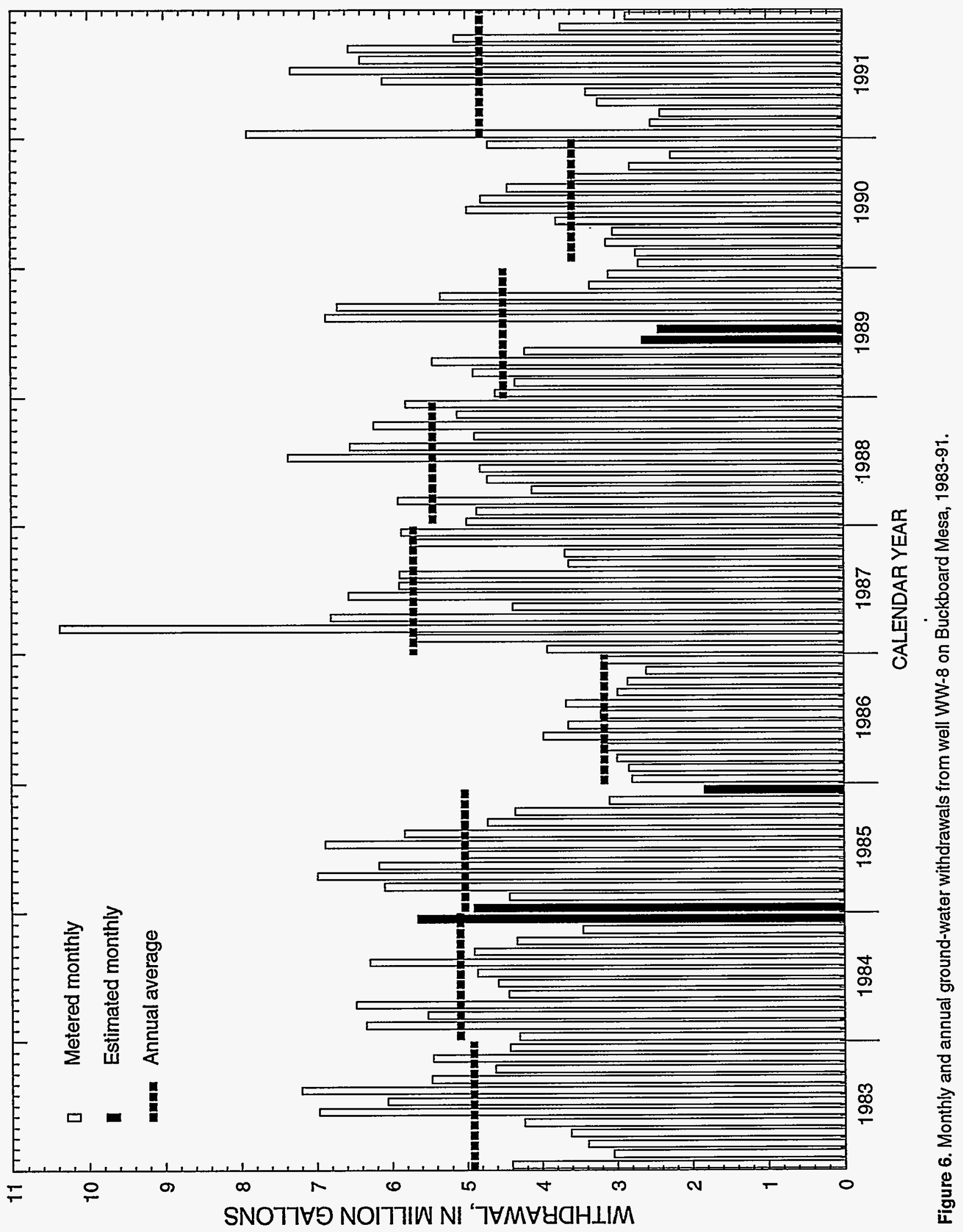




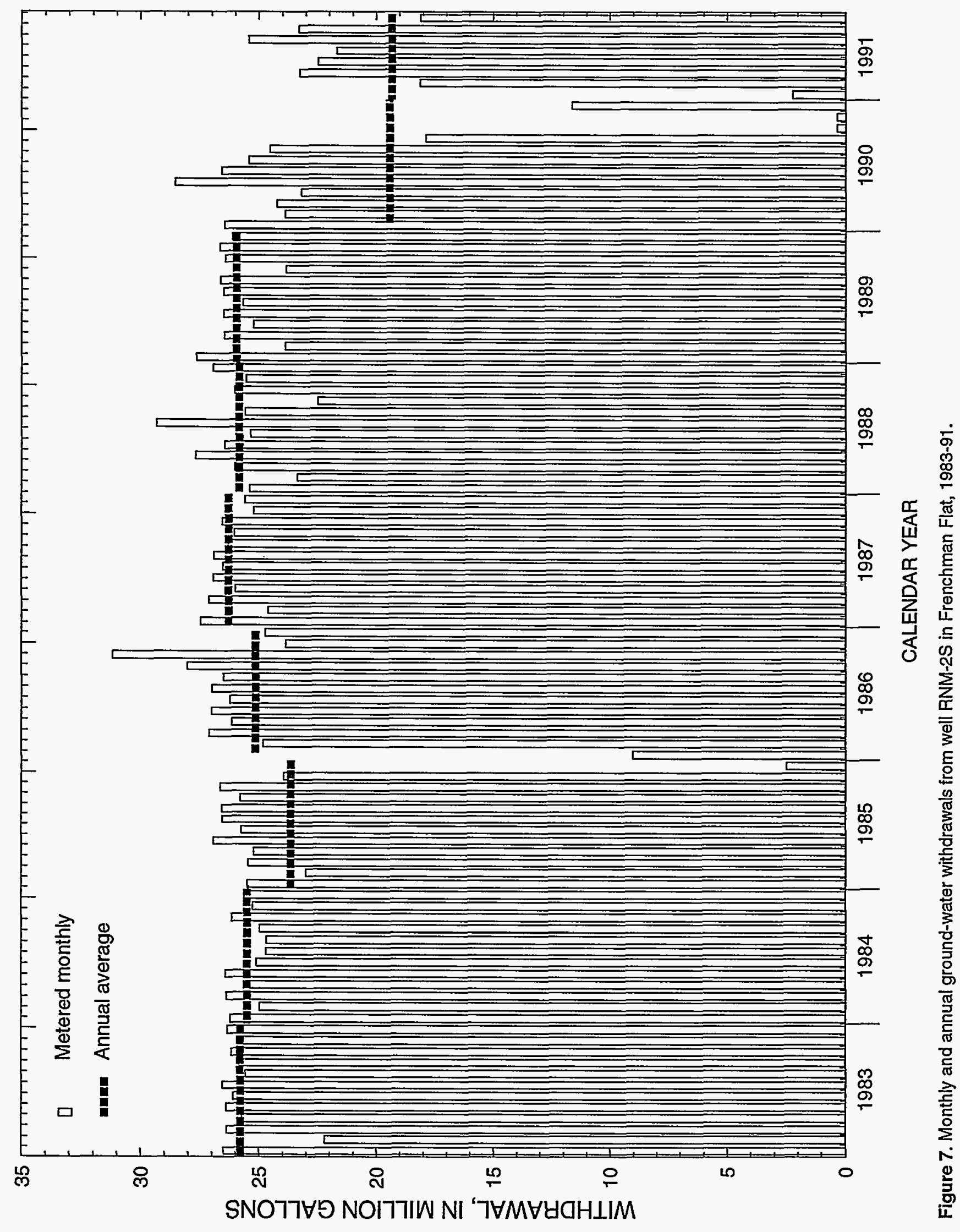

18 Ground-Water Data for 1990-91 and Ground-Water Withdrawals for 1951-91, Nevada Test Slte 


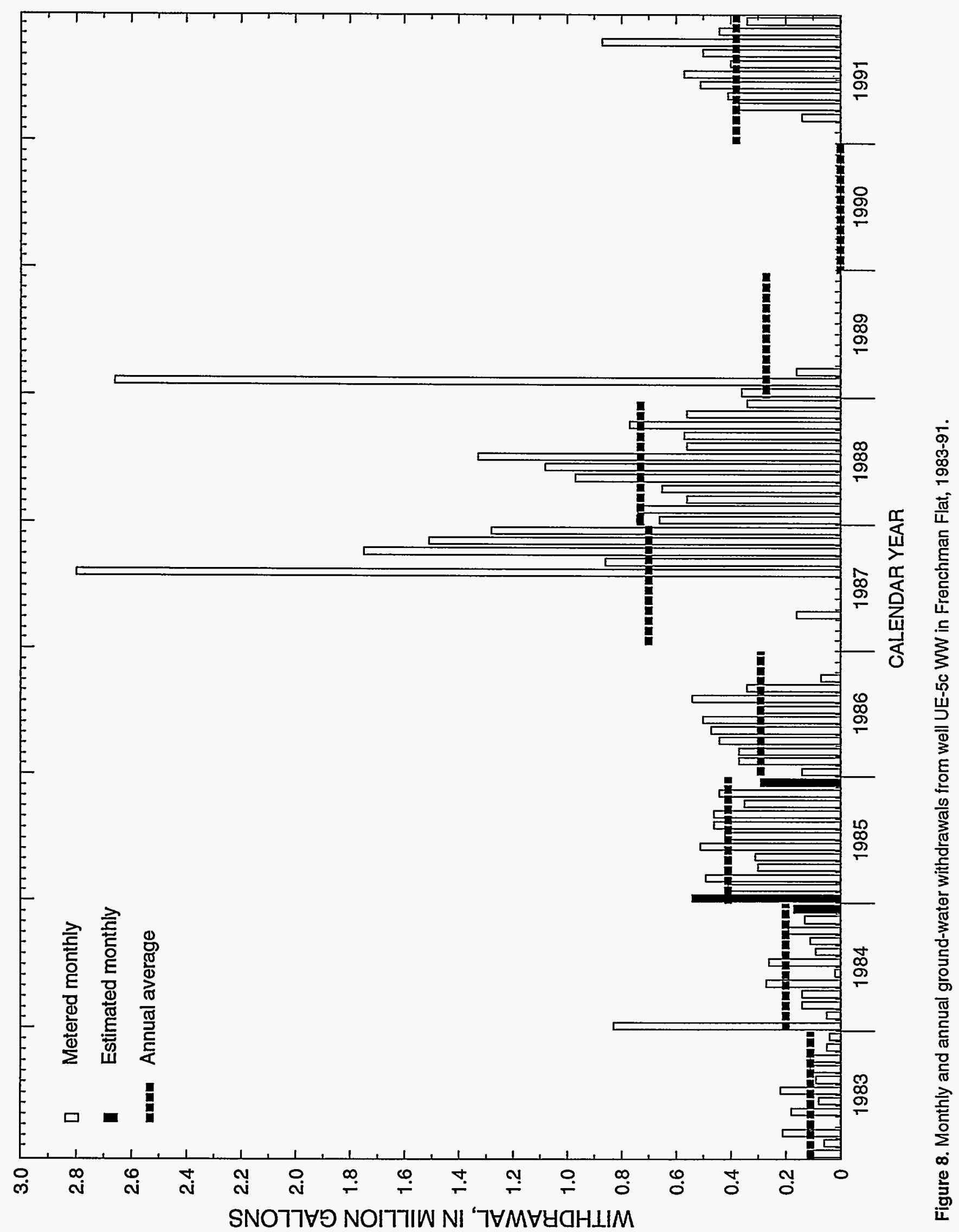

BASIC DATA 


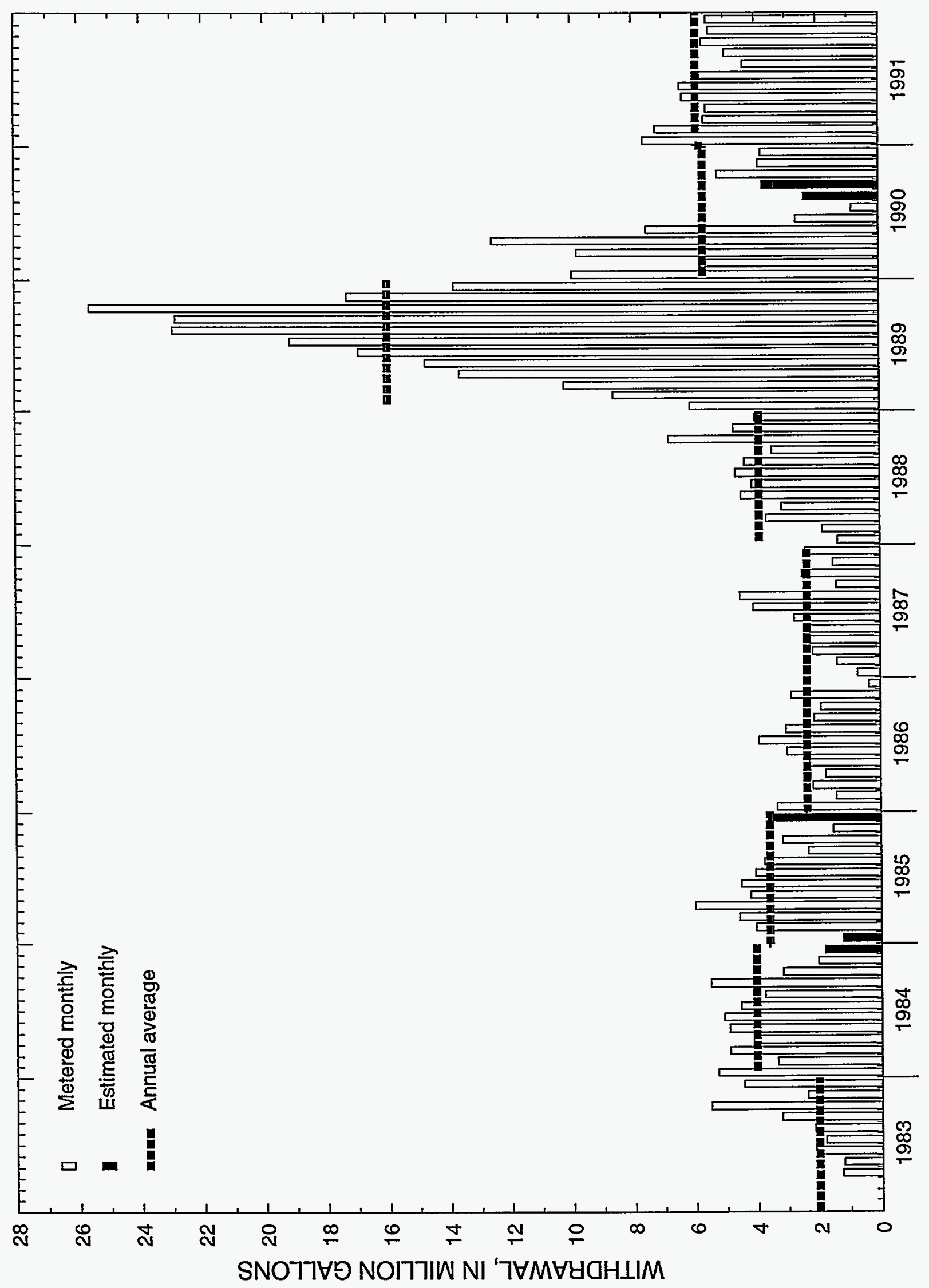

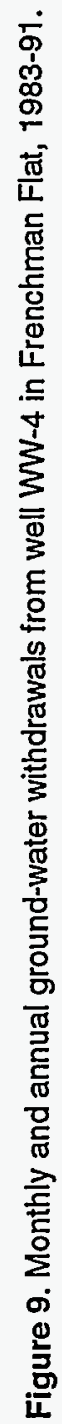




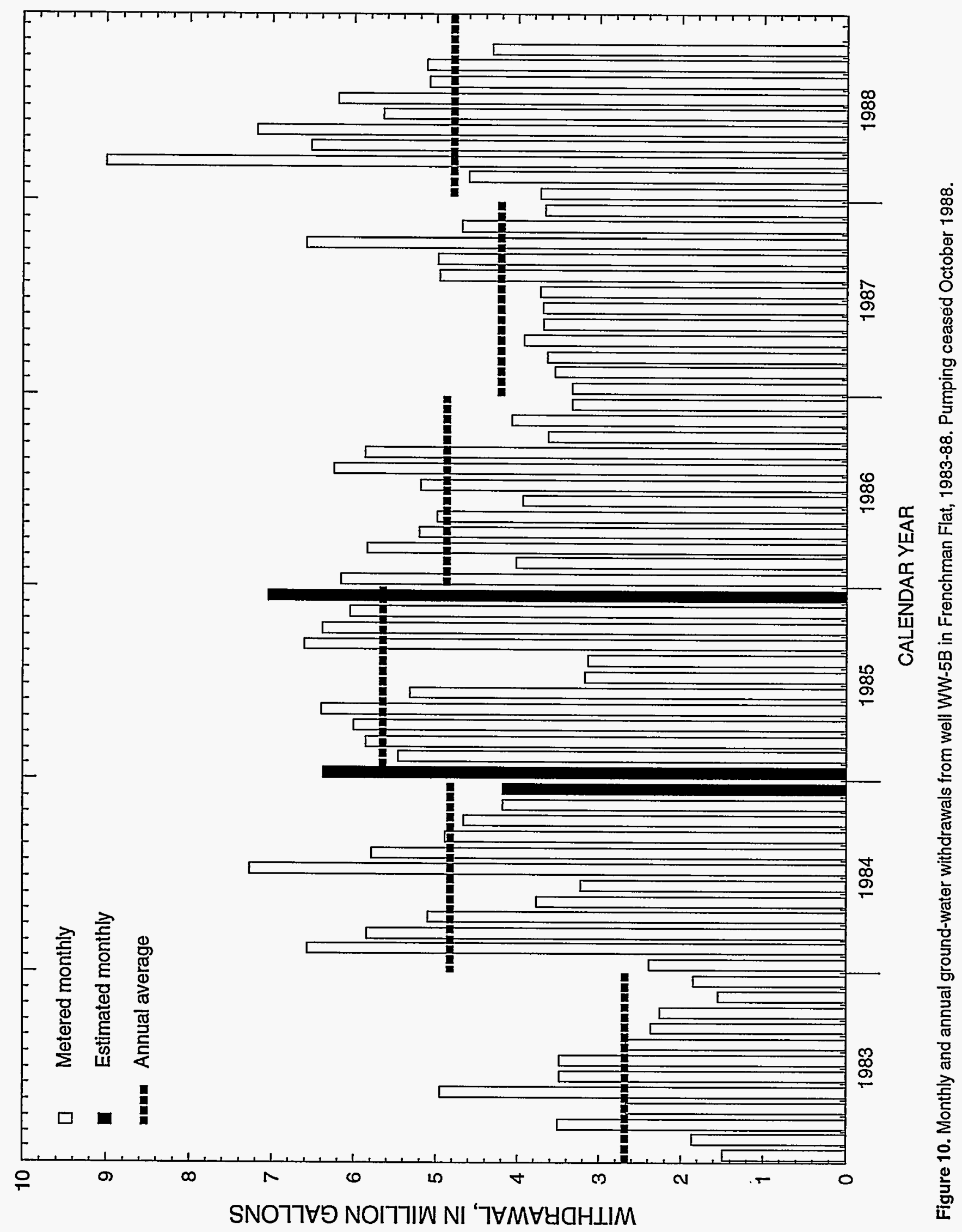




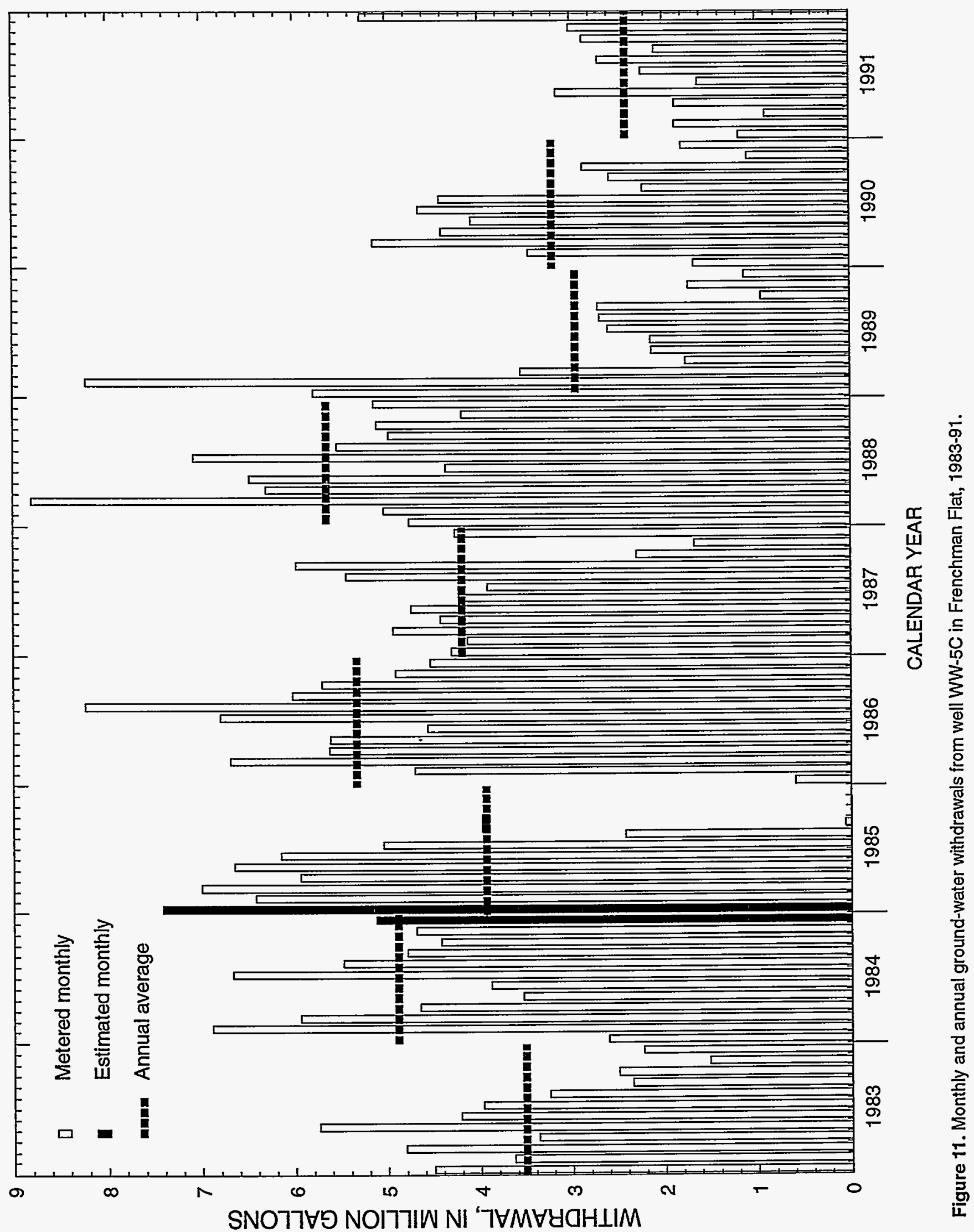

22 Ground-Water Data for 1990-91 and Ground-Water Withdrawals for 1951-91, Nevada Test SIte 


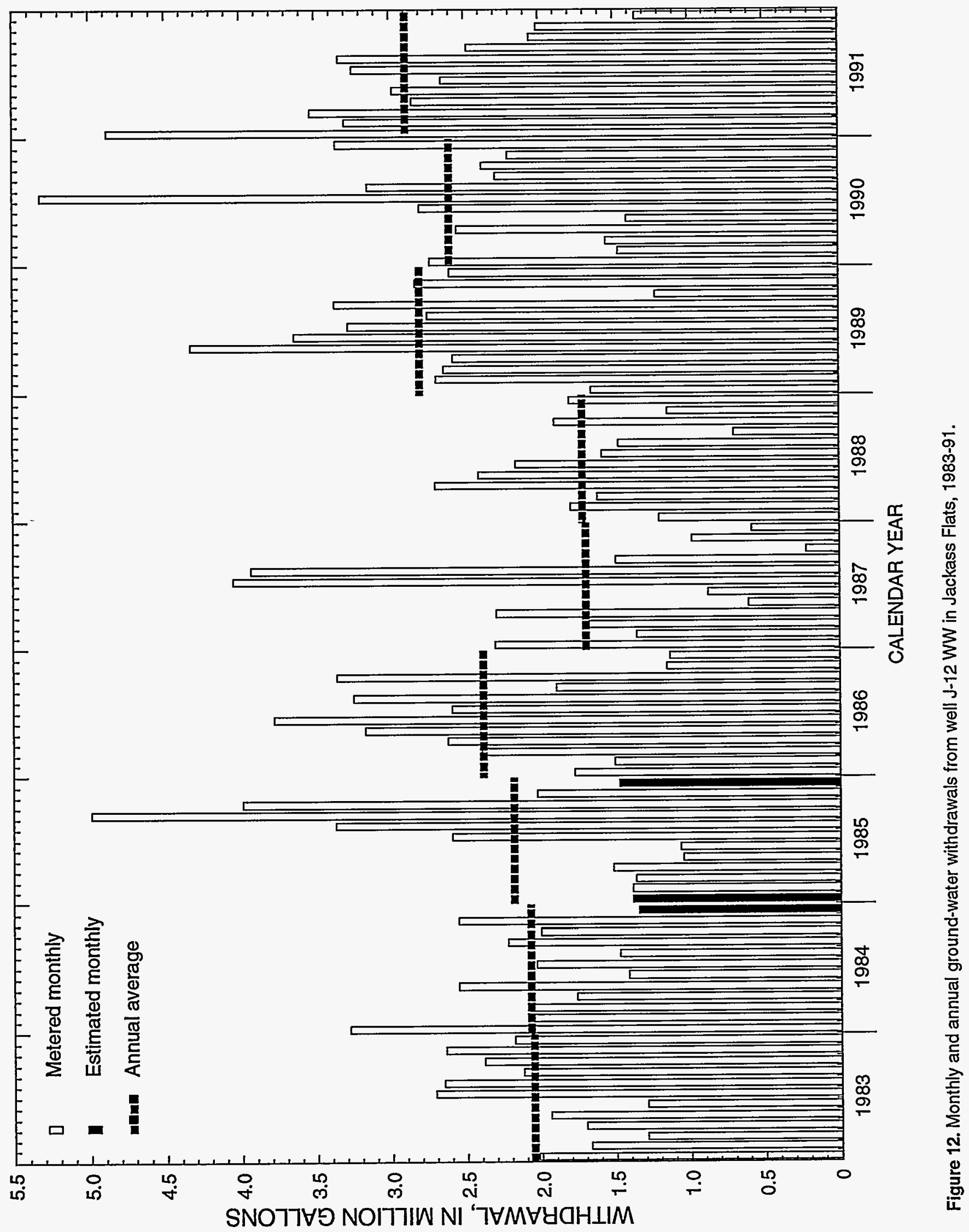




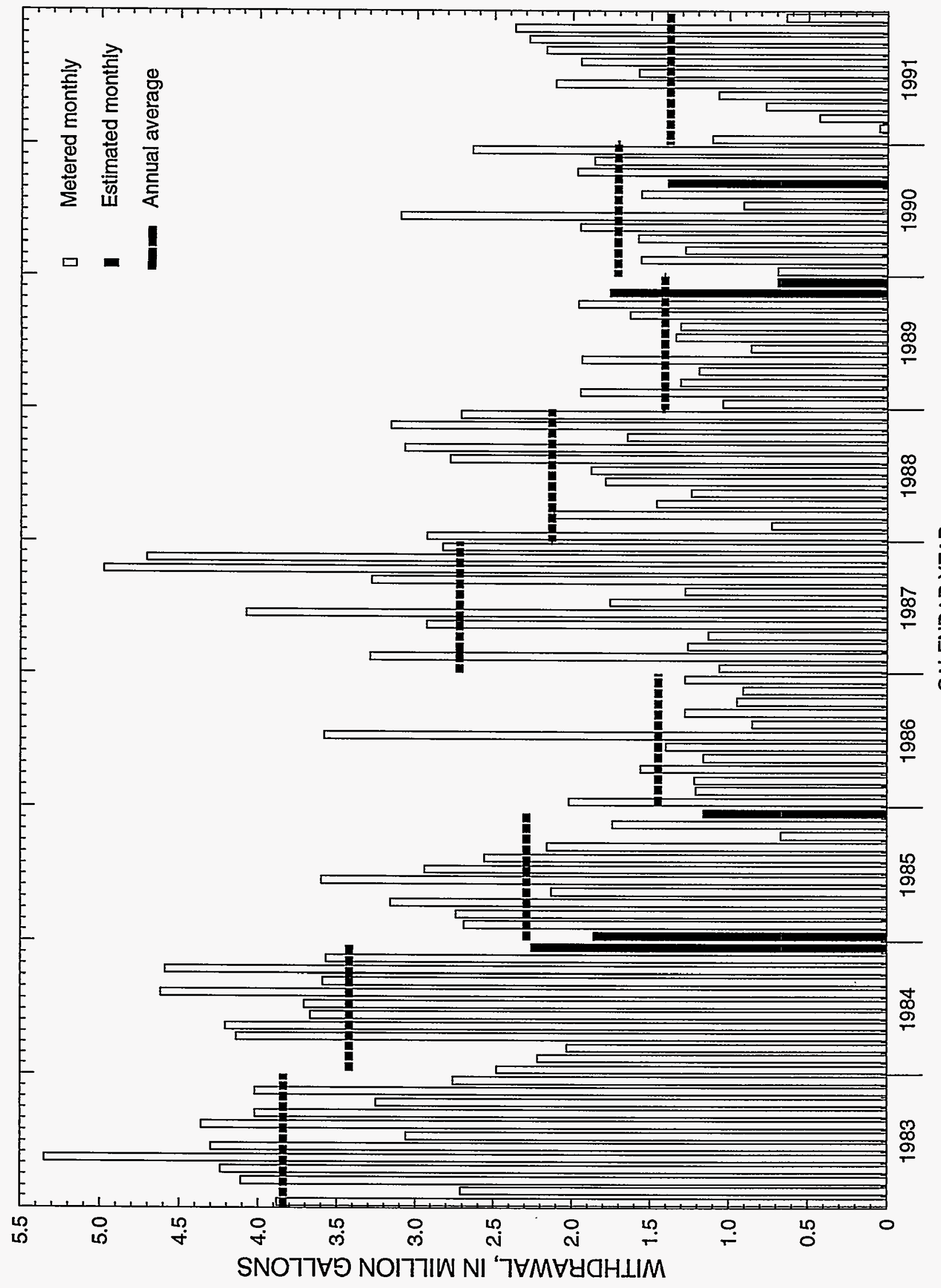

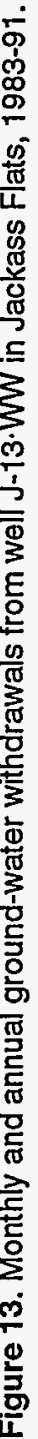




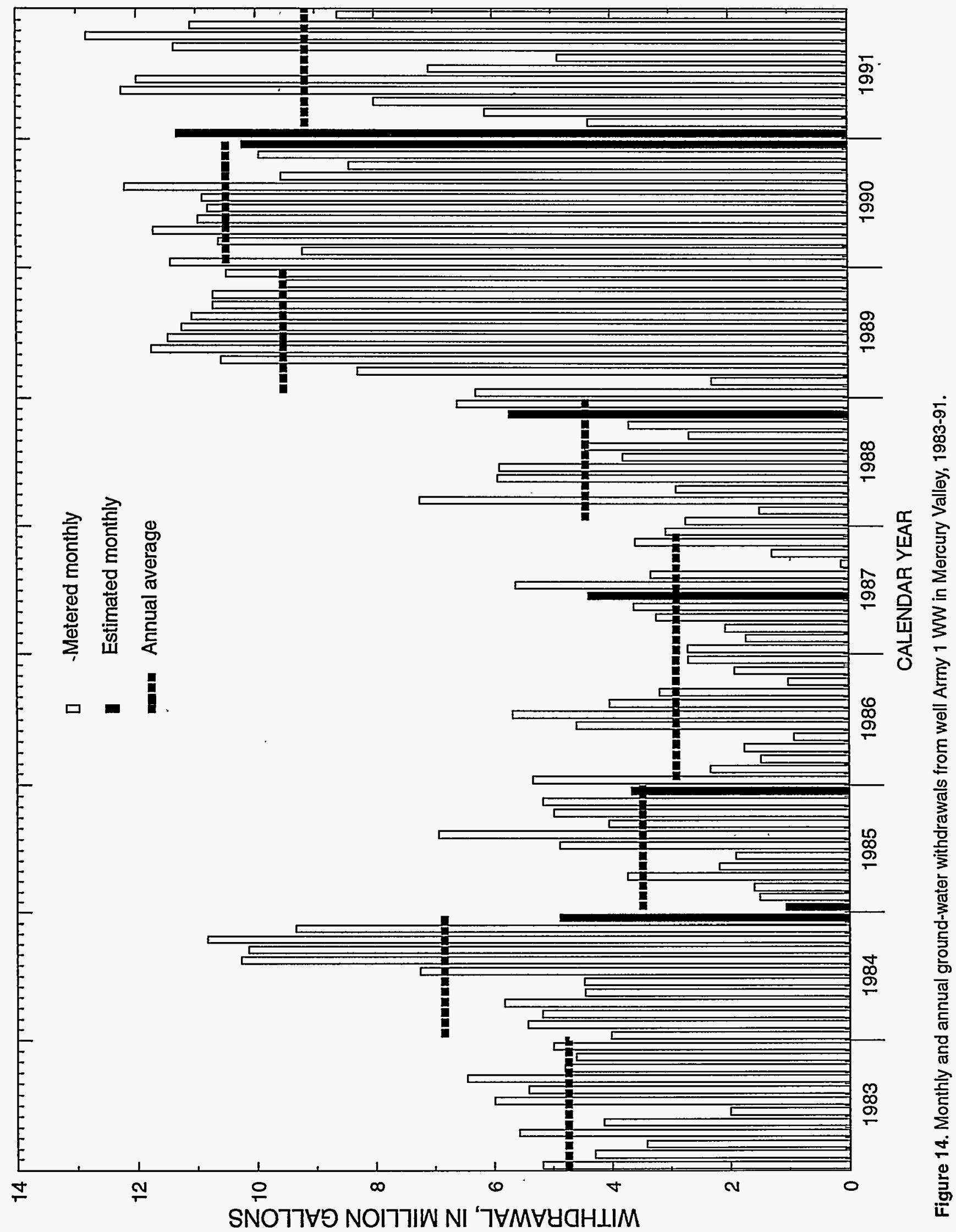




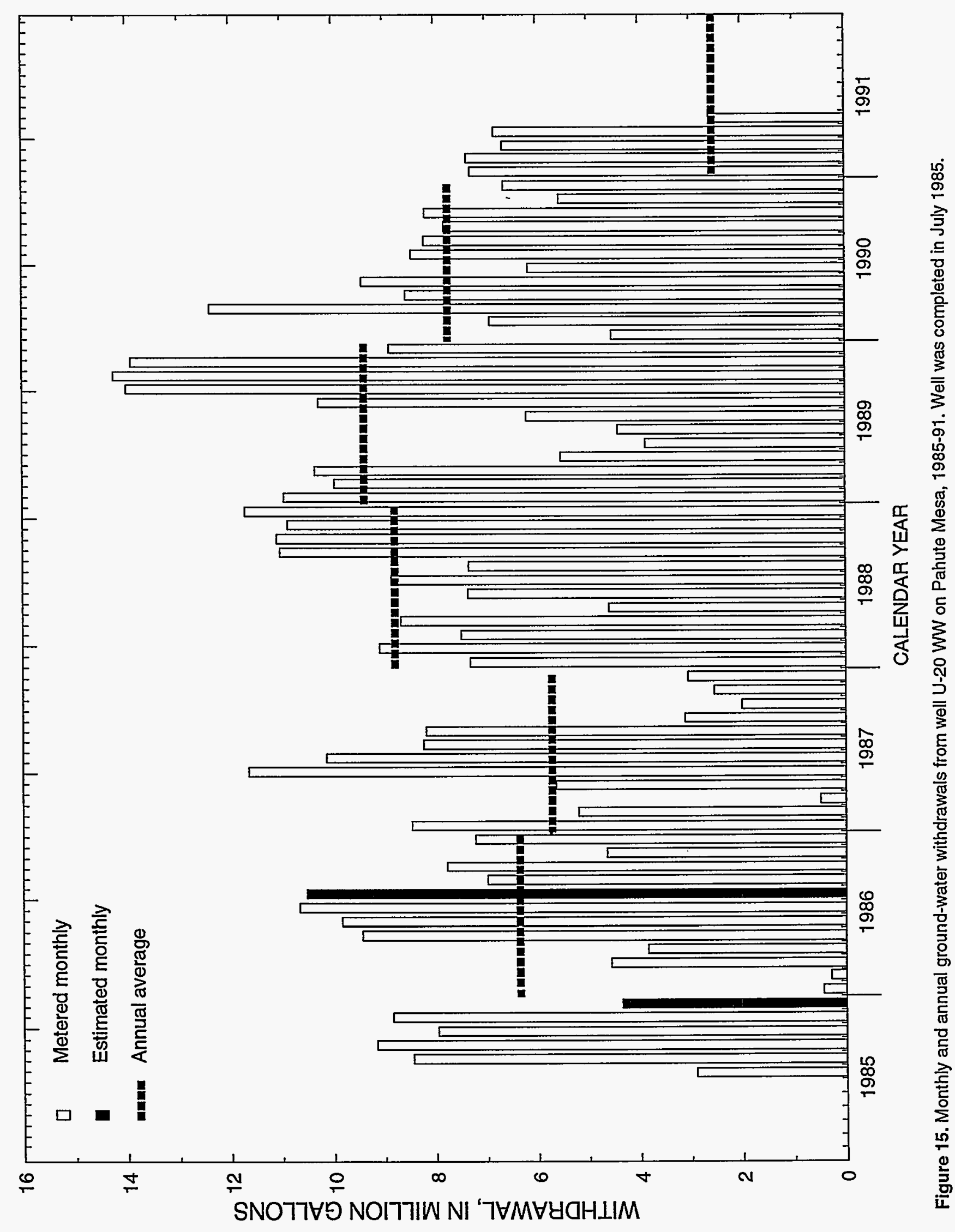




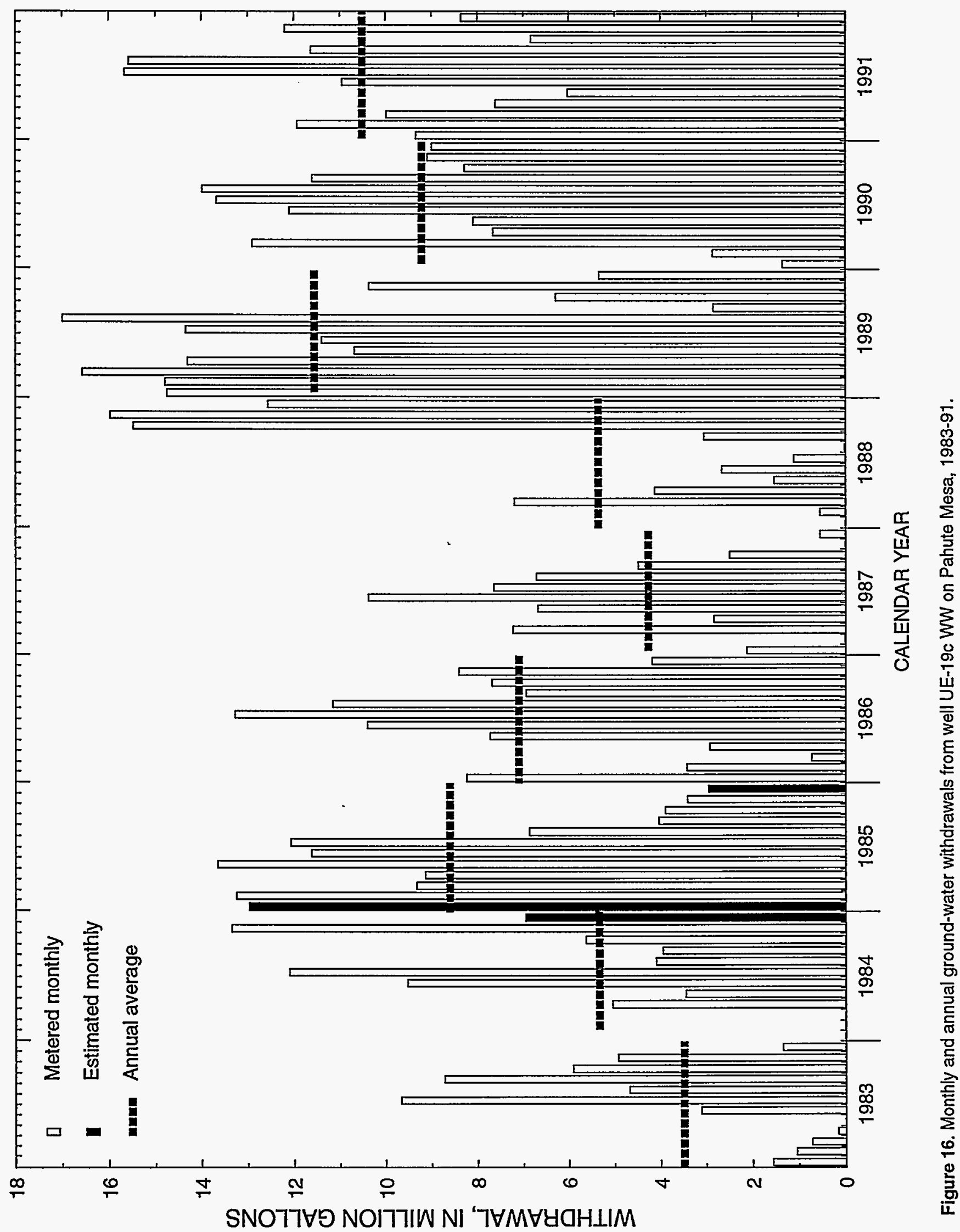




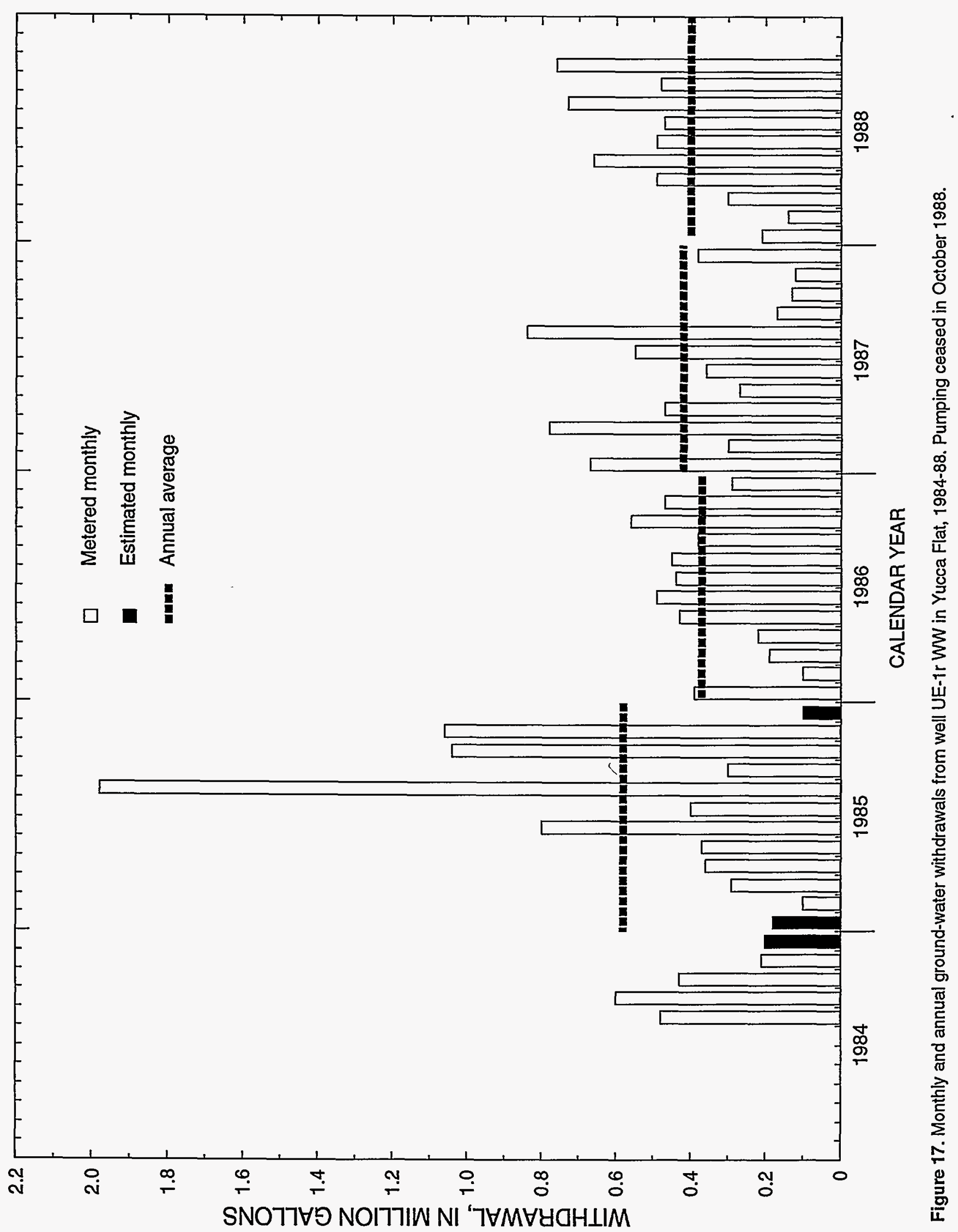




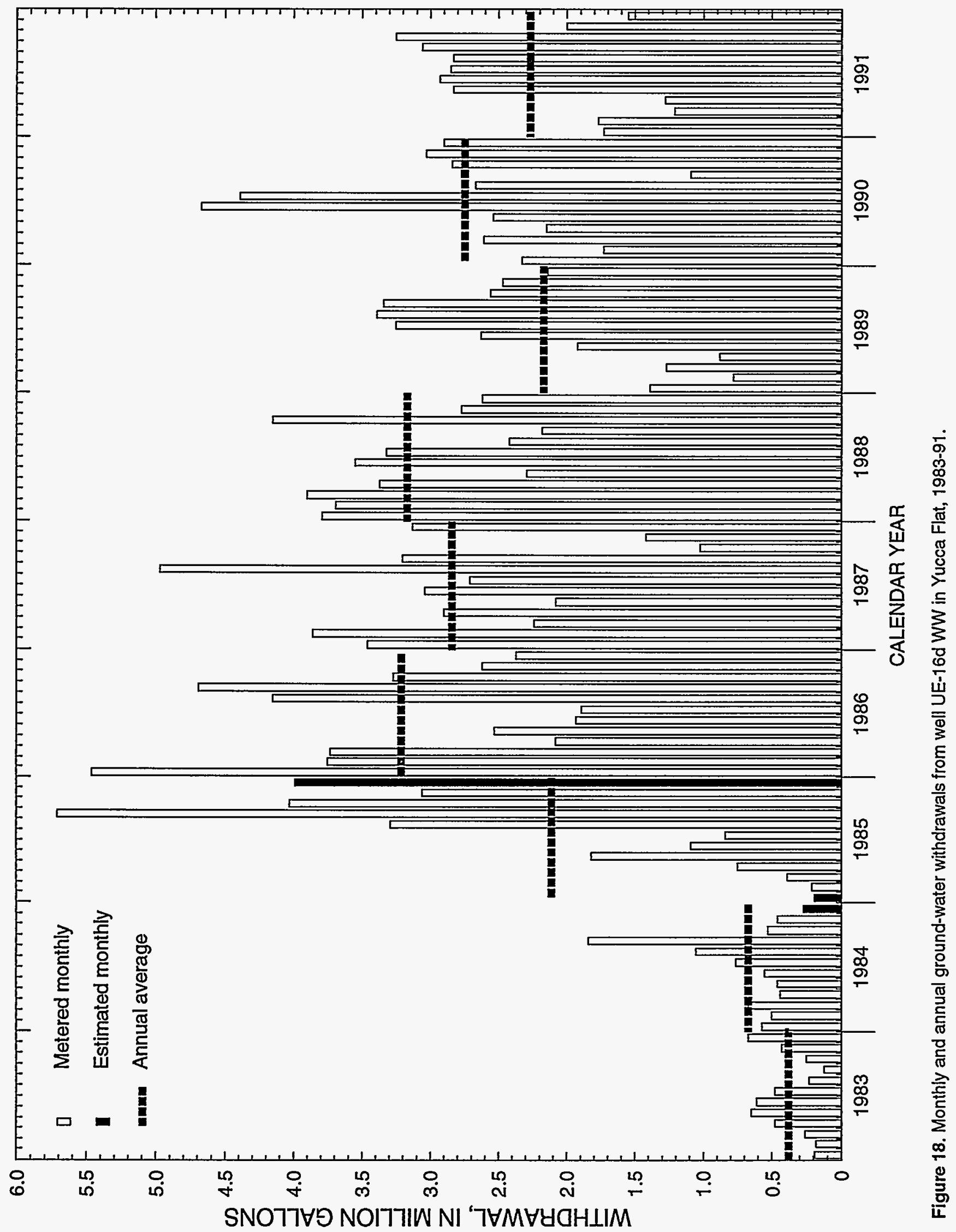




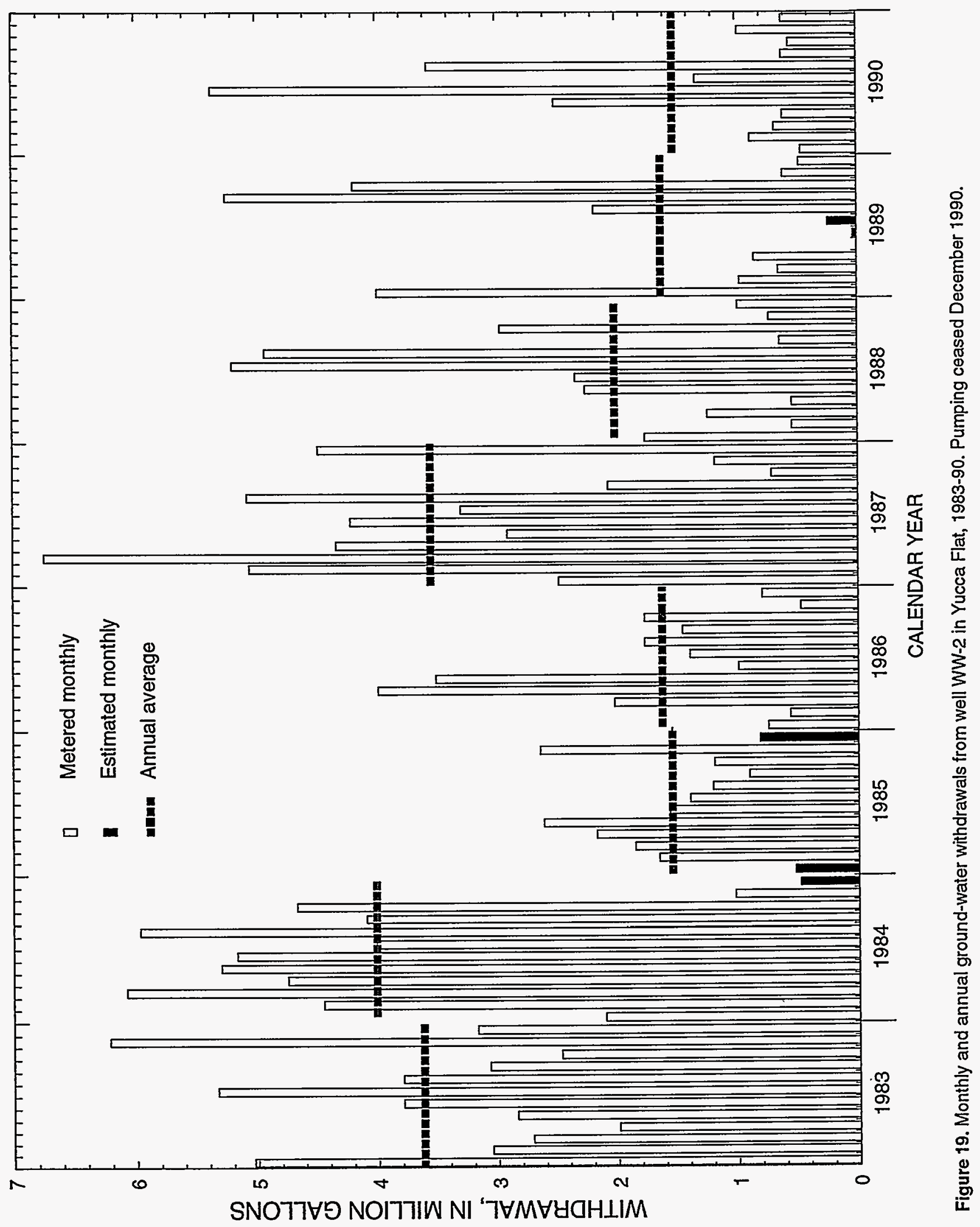




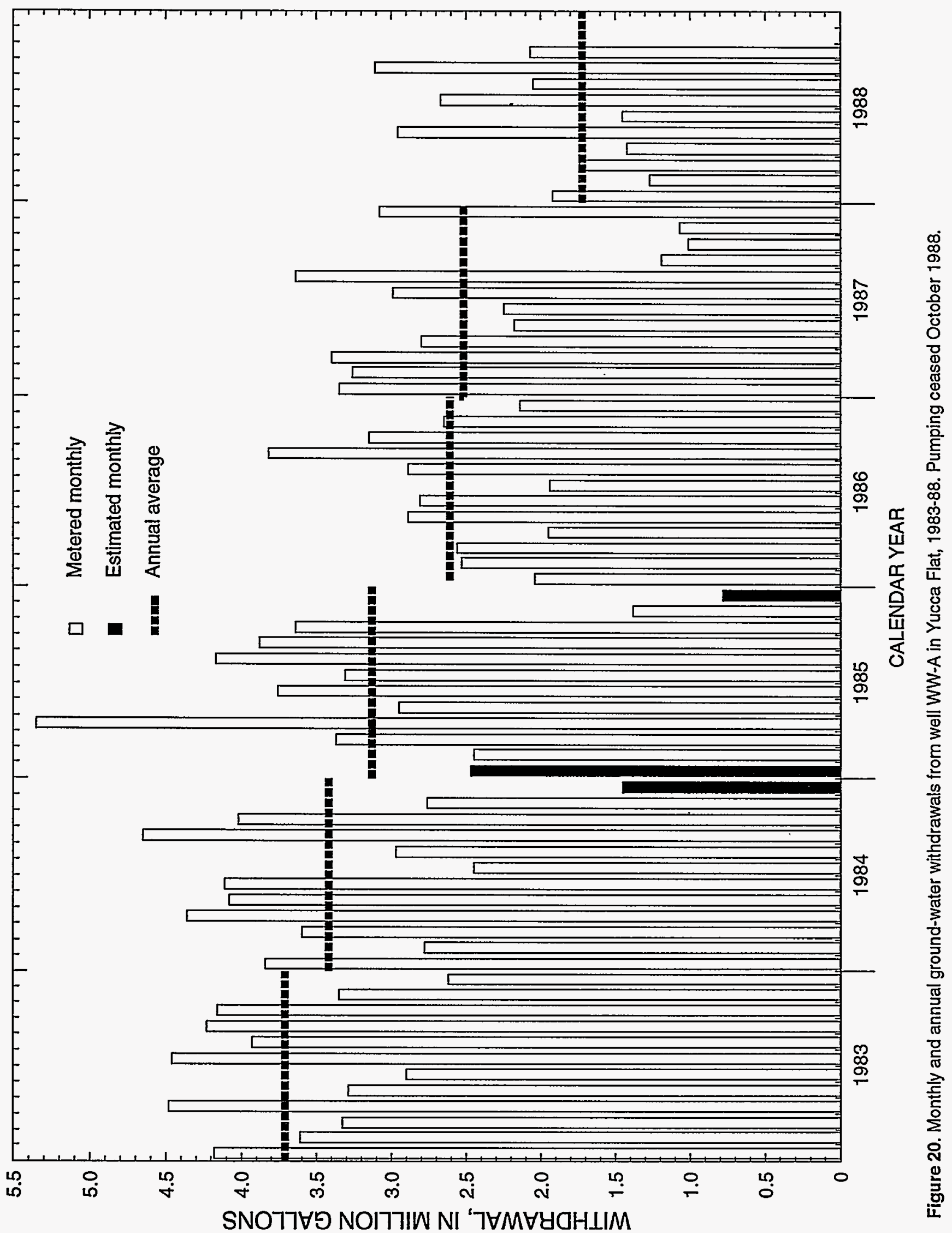




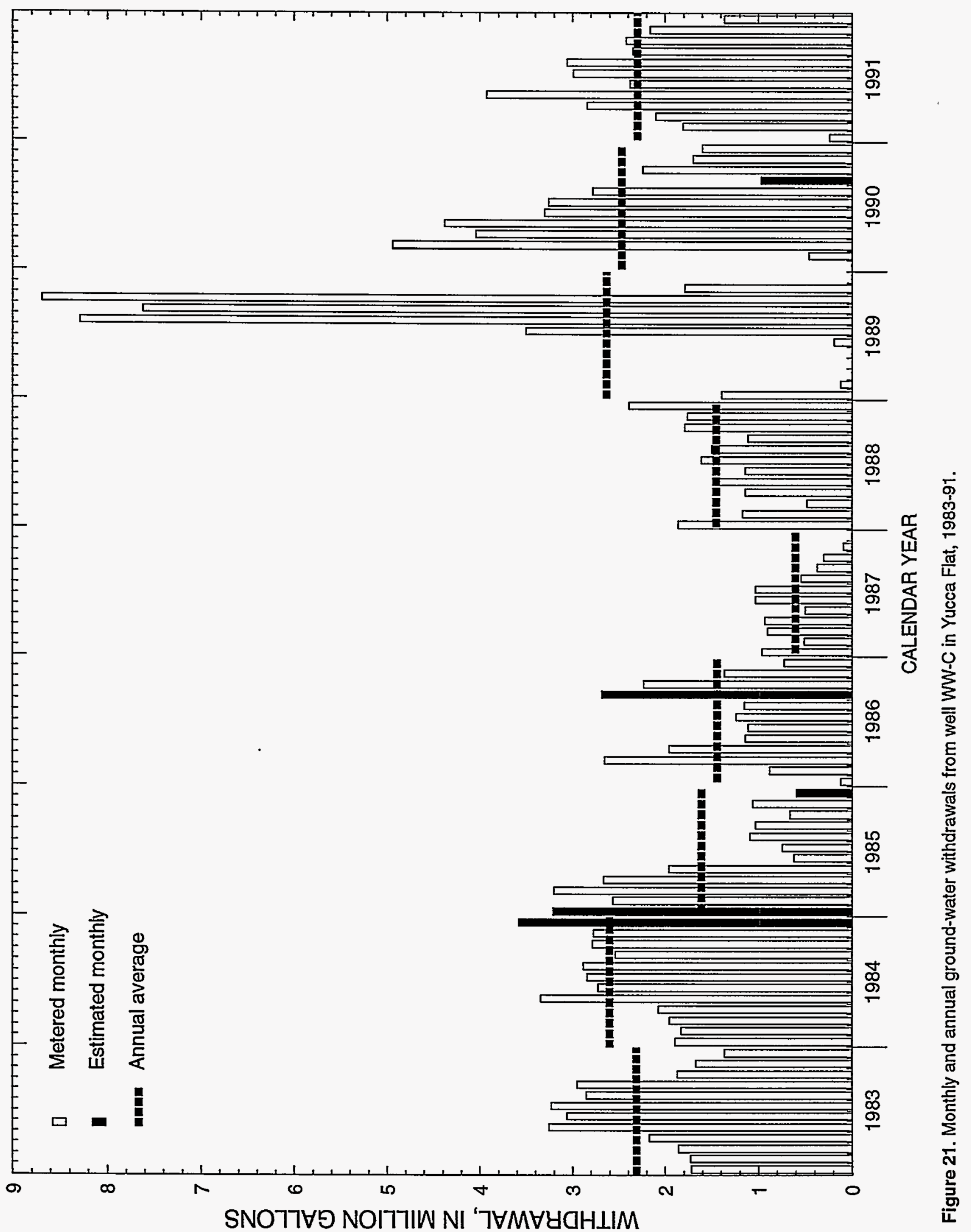




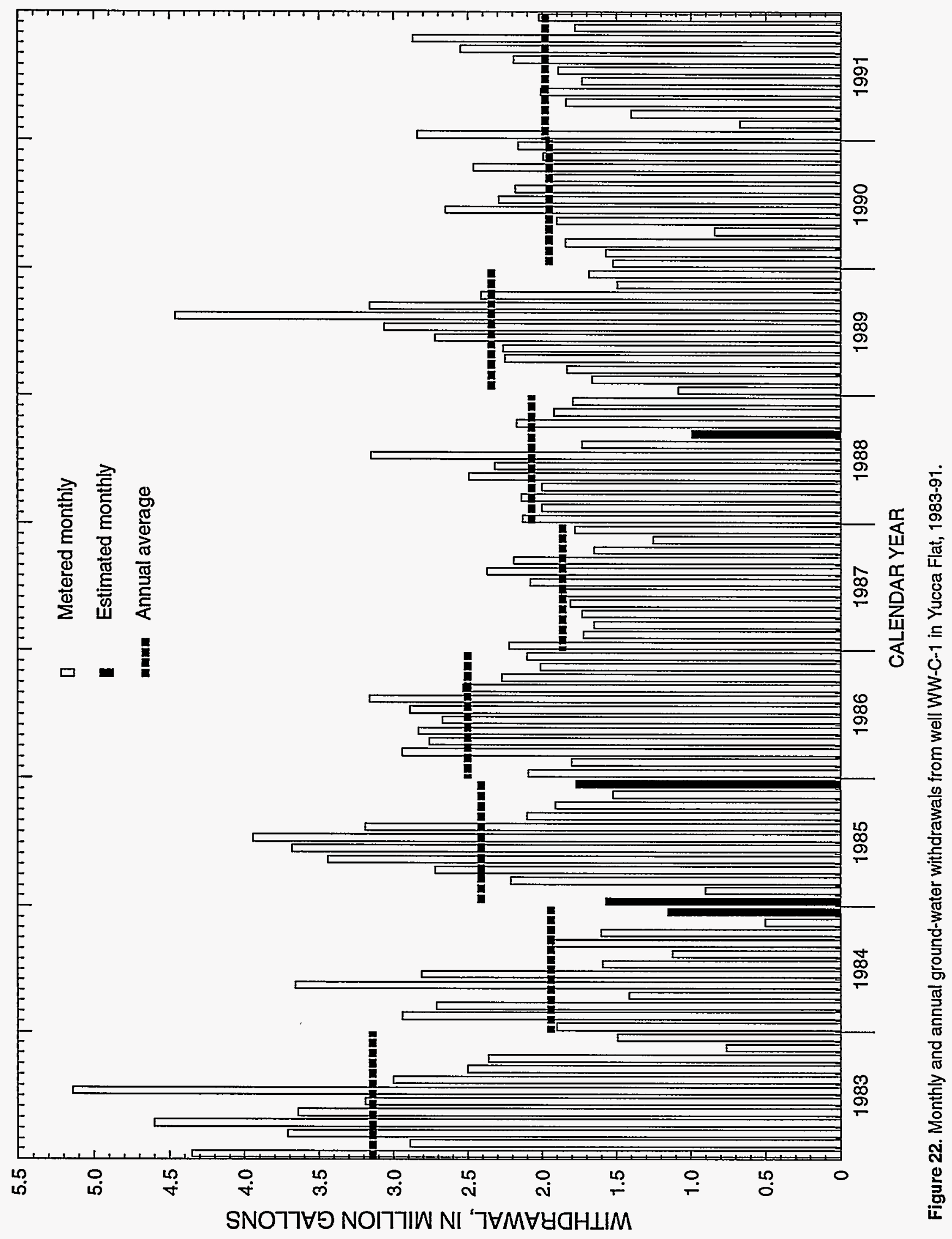


Table 1. Depth to water in wells and test holes at the Nevada Test Site (NTS) and the Faultless Site, Nye County, Nevada, water years 1990-91

NTS hole number--Drill-hole number assigned by Raytheon Services Nevada; see section "Site Designations" in text.

USGS standard Identification--U.S. Geological Survey site designation; see section "Site Designations" in text.

Date hole completed-Date that borehole construction work ceased; sce section "Measurements" in text.

Land-surface altitude--Datum is sea level. Value may not represent current altibude; see section "Depth to Water" in text.

Hole depth--Datum is land surface. Represents most recent available accessible depth; see section "Measurements" in text.

Depth of open intervals--Datum is land surface. Bottom of deepest open interval may be deeper than present accessible hole depth; see section "Measurements" in text. Type: $P$, perforated or slotted casing; $S$, screen; $X$, open (uncased) hole.

Measurement--Datum is land surface. Value may not represent static water level; see section "Depth to Water" in text. Method: $L$, interpreted from geophysical logs; $R$, depth to water reported (measurement method not known); S, steel tape; T, electric tape; V, wire-line or iron-horse device. Site status: D, site was dry-depth to water greater than accessible hole depth; $O$, obstruction was encountered in hole above water surface; $\mathrm{P}$, site was being pumped or $s$ wabbed; $R$, site was recently pumped or swabbed; $S$, nearby site was being pumped or swabbed; $T$, nearby site was recently pumped or swabbed; $Z$, other conditions that may affect the measured depth to water, see section "Measurements" in text.

\begin{tabular}{|c|c|c|c|c|c|c|c|c|c|c|c|c|c|}
\hline \multirow{2}{*}{$\begin{array}{l}\text { NTS hole } \\
\text { number }\end{array}$} & \multirow{2}{*}{$\begin{array}{l}\text { USGS standard } \\
\text { Identification }\end{array}$} & \multirow{2}{*}{$\begin{array}{c}\text { Latitude } \\
\text { (degrees, } \\
\text { minutes, } \\
\text { seconds) }\end{array}$} & \multirow{2}{*}{$\begin{array}{l}\text { Longitude } \\
\text { (degrees, } \\
\text { minutes, } \\
\text { seconds) }\end{array}$} & \multirow{2}{*}{$\begin{array}{l}\text { Date hole } \\
\text { completed } \\
\text { (month, } \\
\text { day, year) }\end{array}$} & \multirow{2}{*}{$\begin{array}{l}\text { Land- } \\
\text { surface } \\
\text { altitude } \\
\text { (feet). }\end{array}$} & \multirow{2}{*}{$\begin{array}{l}\text { Hole } \\
\text { depth } \\
\text { (feet) }\end{array}$} & \multicolumn{3}{|c|}{$\begin{array}{c}\text { Depth of } \\
\text { open Intervals }\end{array}$} & \multicolumn{4}{|c|}{ Measurement } \\
\hline & & & & & & & $\begin{array}{l}\text { Top } \\
\text { (feet) }\end{array}$ & $\begin{array}{c}\text { Bottom } \\
\text { (feet) }\end{array}$ & Type & $\begin{array}{c}\text { Date } \\
\text { (month, } \\
\text { day, year) }\end{array}$ & $\begin{array}{c}\text { Depth to } \\
\text { water } \\
\text { (feet) }\end{array}$ & Method & $\begin{array}{l}\text { Site } \\
\text { status }\end{array}$ \\
\hline \multicolumn{14}{|c|}{ AREA 1} \\
\hline UE-1a & 370254116070601 & 370254 & 1160706 & $02-02-64$ & 4,303 & 562 & $\begin{array}{r}78 \\
947\end{array}$ & $\begin{array}{l}947 \\
957\end{array}$ & $\begin{array}{l}\mathrm{X} \\
\mathrm{X}\end{array}$ & $\begin{array}{l}10-26-89 \\
04-16-90 \\
11-07-90 \\
04-18-91 \\
09-17-91\end{array}$ & $\begin{array}{l}545 \\
545 \\
546 \\
546 \\
546\end{array}$ & $\begin{array}{l}\text { V } \\
\text { V } \\
\text { V } \\
T \\
T\end{array}$ & $\begin{array}{l}- \\
- \\
-\end{array}$ \\
\hline UE-1b & 370254116064201 & 370254 & 1160642 & $02-10-64$ & 4,273 & 701 & $\begin{array}{l}76 \\
80\end{array}$ & $\begin{array}{r}80 \\
1,254\end{array}$ & $\begin{array}{l}X \\
X\end{array}$ & $\begin{array}{l}10-26-89 \\
04-16-90 \\
11-07-90 \\
04-18-91 \\
09-17-91\end{array}$ & $\begin{array}{l}645 \\
644 \\
645 \\
645 \\
645\end{array}$ & $\begin{array}{l}\mathrm{V} \\
\mathrm{V} \\
\mathrm{V} \\
\mathrm{T} \\
\mathrm{T}\end{array}$ & $\begin{array}{l}- \\
- \\
-\end{array}$ \\
\hline UE-1c & 370253116055201 & 370253 & 1160552 & $02-11-64$ & 4,206 & 1,772 & $\begin{array}{l}74 \\
80\end{array}$ & $\begin{array}{r}80 \\
1,880\end{array}$ & $\begin{array}{l}\mathrm{X} \\
\mathrm{X}\end{array}$ & $\begin{array}{l}10-26-89 \\
04-16-90 \\
11-07-90 \\
04-18-91 \\
09-17-91\end{array}$ & $\begin{array}{l}1,297 \\
1,296 \\
1,297 \\
1,296 \\
1,297\end{array}$ & $\begin{array}{l}\mathrm{V} \\
\mathrm{V} \\
\mathrm{V} \\
\mathrm{V} \\
\mathrm{v}\end{array}$ & $\begin{array}{l}- \\
- \\
-\end{array}$ \\
\hline UE-1h & 370005116040301 & 370005 & 1160403 & $07-03-68$ & 3,995 & 3,228 & $\begin{array}{l}2,134 \\
2,349\end{array}$ & $\begin{array}{l}2,349 \\
3,358\end{array}$ & $\begin{array}{l}\mathrm{X} \\
\mathrm{X}\end{array}$ & $\begin{array}{l}10-04-89 \\
11-05-90 \\
04-18-91 \\
09-17-91\end{array}$ & $\begin{array}{l}1,557 \\
1,556 \\
1,556 \\
1,557\end{array}$ & $\begin{array}{l}\mathrm{V} \\
\mathrm{V} \\
\mathrm{V} \\
\mathrm{V}\end{array}$ & $\begin{array}{l}- \\
- \\
-\end{array}$ \\
\hline UE-1L & 370254116082002 & 370254 & 1160820 & $11-11-77$ & 4,454 & 2,284 & $\begin{array}{l}716 \\
726\end{array}$ & $\begin{array}{r}726 \\
2,284\end{array}$ & $\begin{array}{l}\mathrm{X} \\
\mathrm{X}\end{array}$ & $\begin{array}{l}10-17-89 \\
04-13-90 \\
11-07-90 \\
04-18-91 \\
09-17-91\end{array}$ & $\begin{array}{l}516 \\
515 \\
519 \\
519 \\
520\end{array}$ & $\begin{array}{l}V \\
V \\
V \\
T \\
T\end{array}$ & $\begin{array}{l}- \\
- \\
- \\
-\end{array}$ \\
\hline UE-1q & 370337116033001 & 370337 & 1160330 & $11-10-80$ & 4,082 & 2,120 & $\begin{array}{l}78 \\
80\end{array}$ & $\begin{array}{r}80 \\
2,437\end{array}$ & $\begin{array}{l}\mathrm{X} \\
\mathrm{X}\end{array}$ & $\begin{array}{l}10-16-89 \\
04-16-90 \\
11-02-90 \\
04-18-91 \\
09-17-91\end{array}$ & $\begin{array}{l}1,554 \\
1,553 \\
1,553 \\
1,553 \\
1,553\end{array}$ & $\begin{array}{l}\mathrm{V} \\
\mathrm{V} \\
\mathrm{V} \\
\mathrm{V} \\
\mathrm{V}\end{array}$ & :- \\
\hline
\end{tabular}


Table 1. Depth to water in wells and test holes at the Nevada Test Site (NTS) and the Faultless Site, Nye County, Nevada, water years 1990-91--Continued

\begin{tabular}{|c|c|c|c|c|c|c|c|c|c|c|c|c|c|}
\hline \multirow{2}{*}{$\begin{array}{l}\text { NTS hole } \\
\text { number }\end{array}$} & \multirow{2}{*}{$\begin{array}{l}\text { USGS standard } \\
\text { Identification }\end{array}$} & \multirow{2}{*}{$\begin{array}{l}\text { Latitude } \\
\text { (degrees, } \\
\text { minutes, } \\
\text { seconds) }\end{array}$} & \multirow{2}{*}{$\begin{array}{l}\text { Longitude } \\
\text { (degrees, } \\
\text { minutes, } \\
\text { seconds) }\end{array}$} & \multirow{2}{*}{$\begin{array}{l}\text { Date hole } \\
\text { completed } \\
\text { (month, } \\
\text { day, year) }\end{array}$} & \multirow{2}{*}{$\begin{array}{l}\text { Land- } \\
\text { surface } \\
\text { altitude } \\
\text { (feet) }\end{array}$} & \multirow{2}{*}{$\begin{array}{l}\text { Hole } \\
\text { depth } \\
\text { (feet) }\end{array}$} & \multicolumn{3}{|c|}{$\begin{array}{l}\text { Depth of } \\
\text { open intervals }\end{array}$} & \multicolumn{4}{|c|}{ Measurement } \\
\hline & & & & & & & $\begin{array}{c}\text { Top } \\
\text { (feet) }\end{array}$ & $\begin{array}{c}\text { Bottom } \\
\text { (feet) }\end{array}$ & Type & $\begin{array}{c}\text { Date } \\
\text { (month, } \\
\text { day, year) }\end{array}$ & $\begin{array}{l}\text { Depth to } \\
\text { water } \\
\text { (feet) }\end{array}$ & Method & $\begin{array}{l}\text { Site } \\
\text { status }\end{array}$ \\
\hline \multicolumn{14}{|c|}{ AREA 2} \\
\hline \multirow[t]{8}{*}{ UE-2ce ${ }^{1}$} & 370831116080701 & 370831 & 1160807 & $01-23-77$ & 4,764 & 1,649 & $\begin{array}{l}1,384 \\
1,624 \\
1,444 \\
1,445\end{array}$ & $\begin{array}{l}1,624 \\
1,650 \\
1,504 \\
1,505\end{array}$ & $\begin{array}{l}P \\
X \\
P \\
P\end{array}$ & $\begin{array}{l}10-02-89 \\
10-19-89 \\
11-07-89 \\
11-14-89 \\
11-28-89\end{array}$ & $\begin{array}{l}1,449 \\
1,450 \\
1,449 \\
1,450 \\
1,450\end{array}$ & $\begin{array}{l}V \\
V \\
V \\
V \\
V\end{array}$ & $\begin{array}{l}- \\
- \\
- \\
-\end{array}$ \\
\hline & & & & & & & & & & $\begin{array}{l}12-05-89 \\
12-11-89 \\
01-09-90 \\
01-23-90 \\
01-31-90\end{array}$ & $\begin{array}{l}1,450 \\
1,450 \\
1,449 \\
1,449 \\
1,449\end{array}$ & $\begin{array}{l}\mathrm{V} \\
\mathrm{V} \\
\mathrm{V} \\
\mathrm{V} \\
\mathrm{V}\end{array}$ & $\begin{array}{l}- \\
- \\
- \\
-\end{array}$ \\
\hline & & & & & & & & & & $\begin{array}{l}02-05-90 \\
02-06-90 \\
03-02-90 \\
03-20-90 \\
05-01-90 \\
05-09-90\end{array}$ & $\begin{array}{l}1,449 \\
1,449 \\
1,449 \\
1,449 \\
1,448 \\
1,448\end{array}$ & $\begin{array}{l}V \\
V \\
V \\
V \\
V \\
V\end{array}$ & $\begin{array}{l}- \\
- \\
- \\
-\end{array}$ \\
\hline & & & & & & & & & & $\begin{array}{l}05-17-90 \\
05-23-90 \\
05-30-90 \\
06-06-90 \\
06-15-90\end{array}$ & $\begin{array}{l}1,448 \\
1,448 \\
1,448 \\
1,448 \\
1,448\end{array}$ & $\begin{array}{l}\mathrm{V} \\
\mathrm{V} \\
\mathrm{V} \\
\mathrm{V} \\
\mathrm{V}\end{array}$ & $\begin{array}{l}- \\
- \\
- \\
-\end{array}$ \\
\hline & & & & & & & & & & $\begin{array}{l}06-20-90 \\
06-27-90 \\
07-06-90 \\
07-11-90 \\
07-20-90\end{array}$ & $\begin{array}{l}1,448 \\
1,448 \\
1,448 \\
1,448 \\
1,448\end{array}$ & $\begin{array}{l}\mathrm{V} \\
\mathrm{V} \\
\mathrm{V} \\
\mathrm{V} \\
\mathrm{V}\end{array}$ & $\begin{array}{l}- \\
- \\
- \\
-\end{array}$ \\
\hline & & & & & & & & & & $\begin{array}{l}07-30-90 \\
08-14-90 \\
08-27-90 \\
09-11-90 \\
09-14-90\end{array}$ & $\begin{array}{l}1,448 \\
1,448 \\
1,448 \\
1,448 \\
1,448\end{array}$ & $\begin{array}{l}\mathrm{V} \\
\mathrm{V} \\
\mathrm{V} \\
\mathrm{V} \\
\mathrm{V}\end{array}$ & $\begin{array}{l}- \\
- \\
- \\
-\end{array}$ \\
\hline & & & & & & & & & & $\begin{array}{l}09-19-90 \\
09-19-90 \\
09-26-90 \\
10-11-90 \\
10-16-90\end{array}$ & $\begin{array}{l}1,448 \\
1,448 \\
1,448 \\
1,448 \\
1,448\end{array}$ & $\begin{array}{l}\text { V } \\
\text { V } \\
\text { V } \\
\text { V } \\
\text { V }\end{array}$ & $\begin{array}{l}- \\
- \\
- \\
-\end{array}$ \\
\hline & & & & & & & & & & $\begin{array}{l}10-25-90 \\
10-30-90 \\
10-31-90 \\
11-16-90 \\
11-30-90\end{array}$ & $\begin{array}{l}1,448 \\
1,448 \\
1,448 \\
1,448 \\
1,448\end{array}$ & $\begin{array}{l}S \\
V \\
V \\
V \\
V\end{array}$ & $\begin{array}{l}- \\
- \\
- \\
-\end{array}$ \\
\hline
\end{tabular}


Table 1. Depth to water in wells and test holes at the Nevada Test Site (NTS) and the Faultess Site, Nye County, Nevada, water years 1990-91--Continued

\begin{tabular}{|c|c|c|c|c|c|c|c|c|c|c|c|c|c|}
\hline \multirow{2}{*}{$\begin{array}{l}\text { NTS hole } \\
\text { number }\end{array}$} & \multirow{2}{*}{$\begin{array}{l}\text { USGS standard } \\
\text { Identification }\end{array}$} & \multirow{2}{*}{$\begin{array}{l}\text { Latitude } \\
\text { (degrees, } \\
\text { minutes, } \\
\text { seconds) }\end{array}$} & \multirow{2}{*}{$\begin{array}{l}\text { Longitude } \\
\text { (degrees, } \\
\text { minutes, } \\
\text { seconds) }\end{array}$} & \multirow{2}{*}{$\begin{array}{l}\text { Date hole } \\
\text { completed } \\
\text { (month, } \\
\text { day, year) }\end{array}$} & \multirow{2}{*}{$\begin{array}{l}\text { Land- } \\
\text { surface } \\
\text { altitude } \\
\text { (feet) }\end{array}$} & \multirow{2}{*}{$\begin{array}{l}\text { Hole } \\
\text { depth } \\
\text { (feet) }\end{array}$} & \multicolumn{3}{|c|}{$\begin{array}{c}\text { Depth of } \\
\text { open intervals }\end{array}$} & \multicolumn{4}{|c|}{ Measurement } \\
\hline & & & & & & & $\begin{array}{l}\text { Top } \\
\text { (feet) }\end{array}$ & $\begin{array}{c}\text { Bottom } \\
\text { (feet) }\end{array}$ & Type & $\begin{array}{c}\text { Date } \\
\text { (month, } \\
\text { day, year) }\end{array}$ & $\begin{array}{c}\text { Depth to } \\
\text { water } \\
\text { (feet) }\end{array}$ & Method & $\begin{array}{l}\text { Site } \\
\text { status }\end{array}$ \\
\hline \multirow[t]{5}{*}{ UE-2ce ${ }^{1}$} & \multirow[t]{5}{*}{370831116080701} & \multirow[t]{5}{*}{370831} & \multirow[t]{5}{*}{1160807} & \multirow[t]{5}{*}{$01-23-77$} & \multirow[t]{4}{*}{4,764} & \multirow[t]{4}{*}{1,649} & \multirow[t]{4}{*}{$\begin{array}{l}1,384 \\
1,624 \\
1,444 \\
1,445\end{array}$} & \multirow[t]{4}{*}{$\begin{array}{l}1,624 \\
1,650 \\
1,504 \\
1,505\end{array}$} & \multirow[t]{4}{*}{$\begin{array}{l}P \\
X \\
P \\
P\end{array}$} & $\begin{array}{l}12-13-90 \\
01-09-91 \\
01-22-91 \\
01-24-91 \\
02-11-91\end{array}$ & $\begin{array}{l}1,448 \\
1,448 \\
1,448 \\
1,448 \\
1,448\end{array}$ & $\begin{array}{l}\mathrm{V} \\
\mathrm{V} \\
\mathrm{V} \\
\mathrm{V} \\
\mathrm{V}\end{array}$ & $\begin{array}{l}- \\
- \\
- \\
-\end{array}$ \\
\hline & & & & & & & & & & $\begin{array}{l}02-27-91 \\
03-13-91 \\
03-26-91 \\
04-10-91 \\
05-09-91\end{array}$ & $\begin{array}{l}1,447 \\
1,447 \\
1,447 \\
1,447 \\
1,447\end{array}$ & $\begin{array}{l}V \\
S \\
V \\
V \\
V\end{array}$ & $\begin{array}{l}- \\
- \\
- \\
-\end{array}$ \\
\hline & & & & & & & & & & $\begin{array}{l}05-22-91 \\
06-05-91 \\
06-20-91 \\
07-03-91 \\
07-16-91\end{array}$ & $\begin{array}{l}1,447 \\
1,446 \\
1,447 \\
1,447 \\
1,447\end{array}$ & $\begin{array}{l}\mathrm{V} \\
\mathrm{V} \\
\mathrm{V} \\
\mathrm{V} \\
\mathrm{V}\end{array}$ & $\begin{array}{l}- \\
- \\
- \\
-\end{array}$ \\
\hline & & & & & & & & & & $\begin{array}{l}07-30-91 \\
08-21-91 \\
08-29-91 \\
09-16-91\end{array}$ & $\begin{array}{l}1,447 \\
1,447 \\
1,447 \\
1,447\end{array}$ & $\begin{array}{l}\mathrm{V} \\
\mathrm{V} \\
\mathrm{V} \\
\mathrm{V}\end{array}$ & $\begin{array}{l}- \\
- \\
-\end{array}$ \\
\hline & & & & & \multicolumn{2}{|c|}{ AREA 3} & & & & & & & \\
\hline \multirow[t]{4}{*}{$\mathrm{TW}-7^{2}$} & \multirow[t]{4}{*}{370353116020201} & \multirow[t]{4}{*}{370353} & \multirow[t]{4}{*}{1160202} & \multirow[t]{4}{*}{$06-27-54$} & \multirow[t]{4}{*}{4,063} & \multirow[t]{4}{*}{2,239} & \multirow[t]{4}{*}{$\begin{array}{l}1,710 \\
1,925 \\
1,970 \\
1,977\end{array}$} & \multirow[t]{4}{*}{$\begin{array}{l}1,720 \\
1,935 \\
2,014 \\
2,251\end{array}$} & \multirow[t]{4}{*}{$\begin{array}{l}\mathrm{P} \\
\mathrm{P} \\
\mathrm{P} \\
\mathrm{P}\end{array}$} & $\begin{array}{l}10-16-89 \\
07-17-90 \\
08-03-90 \\
08-14-90 \\
08-27-90\end{array}$ & $\begin{array}{l}1,622 \\
1,625 \\
1,625 \\
1,625 \\
1,625\end{array}$ & $\begin{array}{l}\mathrm{V} \\
\mathrm{V} \\
\mathrm{V} \\
\mathrm{V} \\
\mathrm{V}\end{array}$ & $\begin{array}{l}- \\
- \\
-\end{array}$ \\
\hline & & & & & & & & & & $\begin{array}{l}09-14-90 \\
11-15-90 \\
01-09-91 \\
01-24-91 \\
02-19-91\end{array}$ & $\begin{array}{l}1,626 \\
1,626 \\
1,627 \\
1,627 \\
1,628\end{array}$ & $\begin{array}{l}V \\
V \\
V \\
V \\
V\end{array}$ & $\begin{array}{l}- \\
- \\
- \\
-\end{array}$ \\
\hline & & & & & & & & & & $\begin{array}{l}03-18-91 \\
04-19-91 \\
05-16-91 \\
06-12-91 \\
07-10-91\end{array}$ & $\begin{array}{l}1,627 \\
1,627 \\
1,627 \\
1,628 \\
1,628\end{array}$ & $\begin{array}{l}V \\
V \\
V \\
V \\
V\end{array}$ & $\begin{array}{l}- \\
- \\
-\end{array}$ \\
\hline & & & & & & & & & & $\begin{array}{l}08-12-91 \\
09-12-91\end{array}$ & $\begin{array}{l}1,630 \\
1,629\end{array}$ & $\begin{array}{l}\mathrm{V} \\
\mathrm{V}\end{array}$ & - \\
\hline$U-3 \mathrm{cn} 5^{3}$ & 370320116012001 & 370334 & 1160121 & $02-07-66$ & 4,012 & 2,830 & $\begin{array}{l}2,832 \\
2,835 \\
3,028\end{array}$ & $\begin{array}{l}2,835 \\
3,028 \\
3,030\end{array}$ & $\begin{array}{l}X \\
X \\
X\end{array}$ & $\begin{array}{l}10-16-89 \\
04-19-90 \\
07-20-90 \\
11-07-90 \\
01-10-91\end{array}$ & $\begin{array}{l}1,620 \\
1,620 \\
1,621 \\
1,620 \\
1,620\end{array}$ & $\begin{array}{l}\mathrm{V} \\
\mathrm{V} \\
\mathrm{V} \\
\mathrm{V} \\
\mathrm{V}\end{array}$ & $\begin{array}{l}- \\
- \\
-\end{array}$ \\
\hline
\end{tabular}


Table 1. Depth to water in wells and test holes at the Nevada Test Site (NTS) and the Faultless Site, Nye County, Nevada, water years 1990-91-Continued

\begin{tabular}{|c|c|c|c|c|c|c|c|c|c|c|c|c|c|}
\hline \multirow{2}{*}{$\begin{array}{c}\text { NTS hole } \\
\text { number }\end{array}$} & \multirow{2}{*}{$\begin{array}{l}\text { USGS standard } \\
\text { identification }\end{array}$} & \multirow{2}{*}{$\begin{array}{l}\text { Latitude } \\
\text { (degrees, } \\
\text { minutes, } \\
\text { seconds) }\end{array}$} & \multirow{2}{*}{$\begin{array}{l}\text { Longitude } \\
\text { (degrees, } \\
\text { minutes, } \\
\text { seconds) }\end{array}$} & \multirow{2}{*}{$\begin{array}{l}\text { Date hole } \\
\text { completed } \\
\text { (month, } \\
\text { day, year) }\end{array}$} & \multirow{2}{*}{$\begin{array}{l}\text { Land- } \\
\text { surface } \\
\text { altitude } \\
\text { (feet) }\end{array}$} & \multirow{2}{*}{$\begin{array}{l}\text { Hole } \\
\text { depth } \\
\text { (feet) }\end{array}$} & \multicolumn{3}{|c|}{$\begin{array}{c}\text { Depth of } \\
\text { open intervals }\end{array}$} & \multicolumn{4}{|c|}{ Measurement } \\
\hline & & & & & & & $\begin{array}{l}\text { Top } \\
\text { (feet) }\end{array}$ & $\begin{array}{c}\text { Bottom } \\
\text { (feet) }\end{array}$ & Type & $\begin{array}{c}\text { Date } \\
\text { (month, } \\
\text { day, year) }\end{array}$ & $\begin{array}{c}\text { Depth to } \\
\text { water } \\
\text { (feet) }\end{array}$ & Method & $\begin{array}{l}\text { Site } \\
\text { status }\end{array}$ \\
\hline \multirow[t]{2}{*}{$\mathrm{U}-3 \mathrm{cn} 5^{3}$} & 370320116012001 & 370334 & 1160121 & $02-07-66$ & 4,012 & 2,830 & $\begin{array}{l}2,832 \\
2,835 \\
3,028\end{array}$ & $\begin{array}{l}2,835 \\
3,028 \\
3,030\end{array}$ & $\begin{array}{l}X \\
X \\
X\end{array}$ & $\begin{array}{l}01-29-91 \\
02-19-91 \\
03-18-91 \\
04-19-91 \\
05-16-91\end{array}$ & $\begin{array}{l}1,620 \\
1,620 \\
1,620 \\
1,620 \\
1,620\end{array}$ & $\begin{array}{l}\mathrm{V} \\
\mathrm{V} \\
\mathrm{V} \\
\mathrm{V} \\
\mathrm{V}\end{array}$ & $\begin{array}{l}- \\
- \\
- \\
-\end{array}$ \\
\hline & & & & & & & & & & $\begin{array}{l}06-12-91 \\
07-10-91 \\
08-14-91 \\
09-12-91\end{array}$ & $\begin{array}{l}1,622 \\
1,620 \\
1,620 \\
1,620\end{array}$ & $\begin{array}{l}V \\
V \\
V \\
V\end{array}$ & $\begin{array}{l}- \\
- \\
-\end{array}$ \\
\hline \multirow[t]{2}{*}{ U-3kv } & 370020116003701 & 370020 & 1160037 & $08-27-79$ & 3,956 & 1,548 & $\begin{array}{l}117 \\
119\end{array}$ & $\begin{array}{r}119 \\
1,600\end{array}$ & $\begin{array}{l}\mathrm{X} \\
\mathrm{X}\end{array}$ & $\begin{array}{l}04-17-90 \\
05-10-90 \\
07-13-90 \\
10-19-90 \\
01-28-91\end{array}$ & $\begin{array}{l}1,533 \\
1,533 \\
1,534 \\
1,534 \\
1,534\end{array}$ & $\begin{array}{l}\mathrm{V} \\
\mathrm{V} \\
\mathrm{V} \\
\mathrm{V} \\
\mathrm{V}\end{array}$ & $\begin{array}{l}- \\
- \\
-\end{array}$ \\
\hline & & & & & & & & & & $\begin{array}{l}04-17-91 \\
08-13-91\end{array}$ & -- & $\begin{array}{l}\mathrm{V} \\
\mathrm{V}\end{array}$ & $\begin{array}{l}D \\
D\end{array}$ \\
\hline \multirow[t]{2}{*}{$\mathrm{U}-3 \mathrm{mi}$} & 370020115593001 & 370021 & 1155930 & $01-20-86$ & 4,004 & 1,761 & $\begin{array}{l}372 \\
380\end{array}$ & $\begin{array}{r}380 \\
1,794\end{array}$ & $\begin{array}{l}X \\
X\end{array}$ & $\begin{array}{l}05-01-90 \\
07-13-90 \\
11-05-90 \\
01-28-91 \\
04-17-91\end{array}$ & $\begin{array}{l}1,579 \\
1,578 \\
1,577 \\
1,576 \\
1,574\end{array}$ & $\begin{array}{l}\mathrm{V} \\
\mathrm{V} \\
\mathrm{V} \\
\mathrm{V} \\
\mathrm{V}\end{array}$ & $\begin{array}{l}- \\
- \\
- \\
-\end{array}$ \\
\hline & & & & & & & & & & $\begin{array}{l}08-13-91 \\
09-18-91\end{array}$ & $\begin{array}{l}1,573 \\
1,573\end{array}$ & $\begin{array}{l}\mathrm{V} \\
\mathrm{V}\end{array}$ & - \\
\hline \multirow[t]{2}{*}{ U-3mt } & 370348116024301 & 370348 & 1160243 & $05-31-90$ & 4,067 & 1,545 & 110 & 1,550 & $\mathrm{x}$ & $\begin{array}{l}07-01-90 \\
05-21-91 \\
05-29-91 \\
06-03-91\end{array}$ & $\begin{array}{r}-\overline{1} \\
1,537 \\
1,537 \\
1,537\end{array}$ & $\begin{array}{l}\text { L } \\
\text { V } \\
\text { V } \\
\text { V }\end{array}$ & $\begin{array}{l}D \\
- \\
- \\
-\end{array}$ \\
\hline & & & & & & & & & & $\begin{array}{l}06-11-91 \\
06-17-91 \\
06-18-91\end{array}$ & $\begin{array}{l}1,538 \\
1,537 \\
1,537\end{array}$ & $\begin{array}{l}\mathrm{V} \\
\mathrm{V} \\
\mathrm{V}\end{array}$ & $\begin{array}{l}- \\
- \\
-\end{array}$ \\
\hline UE-3e 4 & 370411116025909 & 370411 & 1160259 & $01-23-90$ & 4,082 & 2,300 & $\begin{array}{l}1,436 \\
1,442\end{array}$ & $\begin{array}{l}1,442 \\
2,300\end{array}$ & $\begin{array}{l}\mathrm{X} \\
\mathrm{X}\end{array}$ & $\begin{array}{l}01-24-90 \\
01-25-90 \\
01-30-90 \\
01-31-90 \\
02-06-90 \\
02-26-90\end{array}$ & $\begin{array}{l}1,991 \\
1,785 \\
1,545 \\
1,545 \\
1,543 \\
1,537\end{array}$ & $\begin{array}{l}\mathrm{V} \\
\mathrm{V} \\
\mathrm{V} \\
\mathrm{V} \\
\mathrm{V} \\
\mathrm{V}\end{array}$ & $\begin{array}{l}\mathrm{R} \\
\mathrm{Z} \\
- \\
- \\
- \\
-\end{array}$ \\
\hline UE-3e 4-1 & 370411116025910 & 370411 & 1160259 & $03-19-90$ & 4,082 & 2,181 & 2,150 & 2,171 & $S$ & $\begin{array}{l}03-29-90 \\
03-30-90 \\
04-03-90 \\
05-09-90 \\
05-16-90\end{array}$ & $\begin{array}{l}1,083 \\
1,080 \\
1,136 \\
1,117 \\
1,093\end{array}$ & $\begin{array}{l}\mathrm{V} \\
\mathrm{V} \\
\mathrm{V} \\
\mathrm{V} \\
\mathrm{V}\end{array}$ & $\begin{array}{l}\mathrm{Z} \\
- \\
\mathrm{R} \\
- \\
-\end{array}$ \\
\hline
\end{tabular}


Table 1. Depth to water in wells and test holes at the Nevada Test Site (NTS) and the Faultless Site, Nye County, Nevada, water years 1990-91--Continued

\begin{tabular}{|c|c|c|c|c|c|c|c|c|c|c|c|c|c|}
\hline \multirow{2}{*}{$\begin{array}{l}\text { NTS hole } \\
\text { number }\end{array}$} & \multirow{2}{*}{$\begin{array}{l}\text { USGS standard } \\
\text { Identification }\end{array}$} & \multirow{2}{*}{$\begin{array}{c}\text { Latitude } \\
\text { (degrees, } \\
\text { minutes, } \\
\text { seconds) }\end{array}$} & \multirow{2}{*}{$\begin{array}{l}\text { Longitude } \\
\text { (degrees, } \\
\text { minutes, } \\
\text { seconds) }\end{array}$} & \multirow{2}{*}{$\begin{array}{c}\text { Date hole } \\
\text { completed } \\
\text { (month, } \\
\text { day, year) }\end{array}$} & \multirow{2}{*}{$\begin{array}{l}\text { Land- } \\
\text { surface } \\
\text { altitude } \\
\text { (feet) }\end{array}$} & \multirow{2}{*}{$\begin{array}{l}\text { Hole } \\
\text { depth } \\
\text { (feet) }\end{array}$} & \multicolumn{3}{|c|}{$\begin{array}{c}\text { Depth of } \\
\text { open Intervals }\end{array}$} & \multicolumn{4}{|c|}{ Measurement } \\
\hline & & & & & & & $\begin{array}{l}\text { Top } \\
\text { (feet) }\end{array}$ & $\begin{array}{c}\text { Bottom } \\
\text { (feet) }\end{array}$ & Type & $\begin{array}{c}\text { Date } \\
\text { (month, } \\
\text { day, year) }\end{array}$ & $\begin{array}{l}\text { Depth to } \\
\text { water } \\
\text { (feet) }\end{array}$ & Method & $\begin{array}{l}\text { Site } \\
\text { status }\end{array}$ \\
\hline \multirow[t]{8}{*}{ UE-3e 4-1 } & 370411116025910 & 370411 & 1160259 & $03-19-90$ & 4,082 & 2,181 & 2,150 & 2,171 & $S$ & $\begin{array}{l}05-23-90 \\
05-30-90 \\
06-12-90 \\
06-20-90 \\
06-27-90\end{array}$ & $\begin{array}{l}1,069 \\
1,071 \\
1,104 \\
1,085 \\
1,082\end{array}$ & $\begin{array}{l}\mathrm{V} \\
\mathrm{V} \\
\mathrm{V} \\
\mathrm{V} \\
\mathrm{V}\end{array}$ & $\begin{array}{l}- \\
- \\
\mathrm{R} \\
\mathrm{R}\end{array}$ \\
\hline & & & & & & & & & & $\begin{array}{l}07-05-90 \\
07-11-90 \\
07-17-90 \\
07-20-90 \\
08-03-90 \\
08-13-90\end{array}$ & $\begin{array}{l}1,079 \\
1,090 \\
1,094 \\
1,091 \\
1,304 \\
1,087\end{array}$ & $\begin{array}{l}\text { V } \\
\text { V } \\
\text { V } \\
\text { V } \\
\text { V } \\
\text { v }\end{array}$ & $\begin{array}{l}- \\
\vdots \\
\text { R } \\
-\end{array}$ \\
\hline & & & & & & & & & & $\begin{array}{l}08-17-90 \\
08-28-90 \\
09-12-90 \\
09-24-90 \\
10-05-90\end{array}$ & $\begin{array}{l}1,081 \\
1,065 \\
1,057 \\
1,065 \\
1,067\end{array}$ & $\begin{array}{l}\mathrm{V} \\
\mathrm{V} \\
\mathrm{V} \\
\mathrm{v} \\
\mathrm{V}\end{array}$ & $\begin{array}{l}- \\
- \\
-\end{array}$ \\
\hline & & & & & & & & & & $\begin{array}{l}10-23-90 \\
11-15-90 \\
11-30-90 \\
12-18-90 \\
01-07-91\end{array}$ & $\begin{array}{l}1,053 \\
1,049 \\
1,048 \\
1,058 \\
1,050\end{array}$ & $\begin{array}{l}V \\
V \\
V \\
V \\
V\end{array}$ & $\begin{array}{l}- \\
\bar{R} \\
-\end{array}$ \\
\hline & & & & & & & & & & $\begin{array}{l}01-22-91 \\
02-11-91 \\
02-25-91 \\
03-14-91 \\
03-25-91\end{array}$ & $\begin{array}{l}1,050 \\
1,045 \\
1,041 \\
1,032 \\
1,038\end{array}$ & $\begin{array}{l}\mathrm{V} \\
\mathrm{V} \\
\mathrm{V} \\
\mathrm{V} \\
\mathrm{V}\end{array}$ & $\begin{array}{l}- \\
- \\
-\end{array}$ \\
\hline & & & & & & & & & & $\begin{array}{l}04-09-91 \\
04-22-91 \\
05-08-91 \\
05-21-91 \\
06-03-91\end{array}$ & $\begin{array}{l}1,038 \\
1,037 \\
1,038 \\
1,024 \\
1,026\end{array}$ & $\begin{array}{l}\mathrm{V} \\
\mathrm{V} \\
\mathrm{V} \\
\mathrm{V} \\
\mathrm{V}\end{array}$ & $\begin{array}{l}- \\
\vdots \\
-\end{array}$ \\
\hline & & & & & & & & & & $\begin{array}{l}06-17-91 \\
06-19-91 \\
07-03-91 \\
07-15-91 \\
07-31-91\end{array}$ & $\begin{array}{l}1,045 \\
1,045 \\
1,044 \\
1,046 \\
1,044\end{array}$ & $\begin{array}{l}\mathrm{V} \\
\mathrm{V} \\
\mathrm{V} \\
\mathrm{V} \\
\mathrm{V}\end{array}$ & $\begin{array}{l}: \\
:\end{array}$ \\
\hline & & & & & & & & & & $\begin{array}{l}08-12-91 \\
08-21-91 \\
08-29-91\end{array}$ & $\begin{array}{l}1,048 \\
1,041 \\
1,042\end{array}$ & $\begin{array}{l}\mathrm{V} \\
\mathrm{V} \\
\mathrm{V}\end{array}$ & 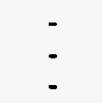 \\
\hline
\end{tabular}


Table 1. Depth to water in wells and test holes at the Nevada Test Site (NTS) and the Faultless Site, Nye County, Nevada, water years 1990-91-Continued

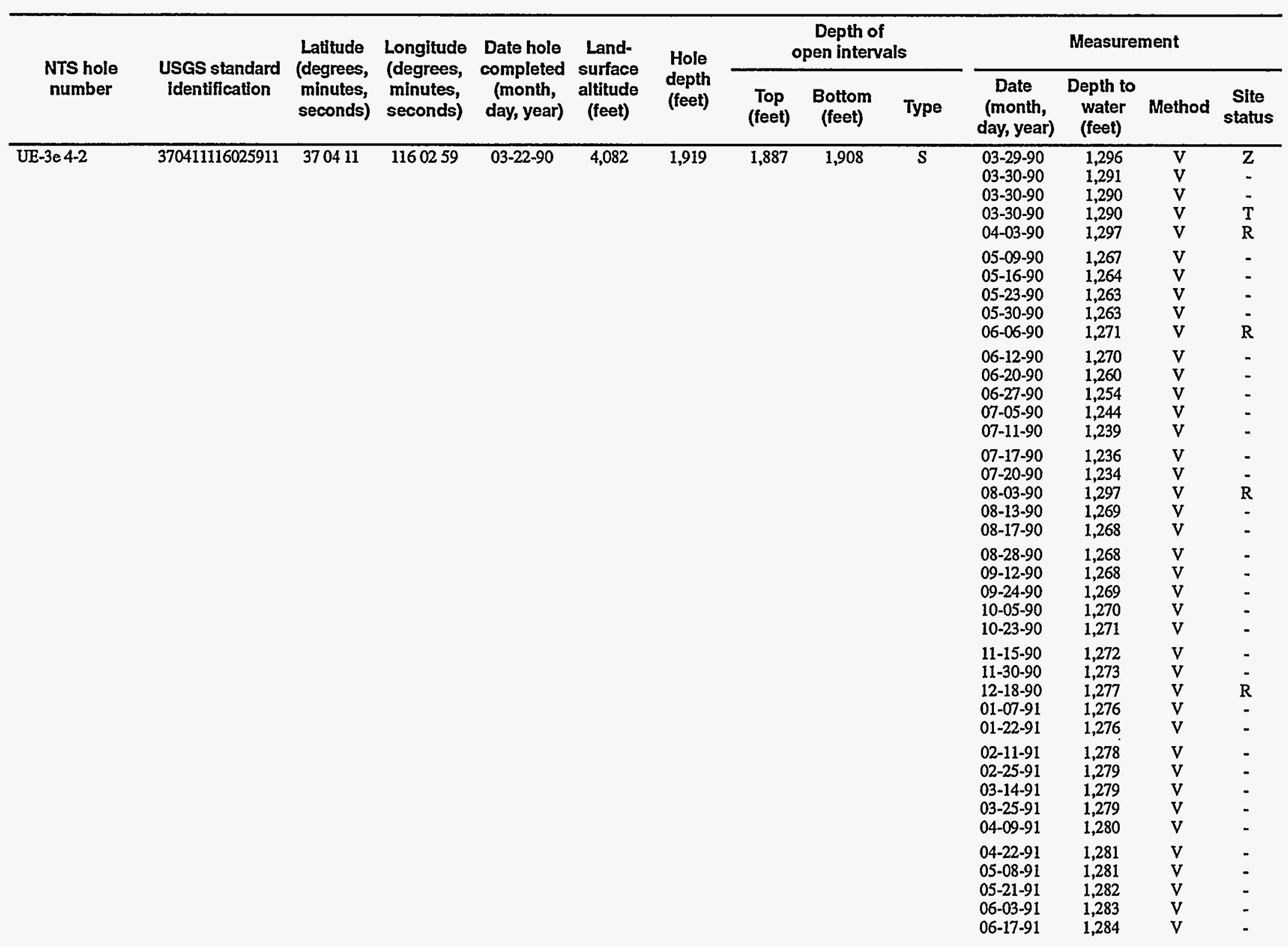


Table 1. Depth to water in wells and test holes at the Nevada Test Site (NTS) and the Faultless Site, Nye County, Nevada, water years 1990-91--Continued

\begin{tabular}{|c|c|c|c|c|c|c|c|c|c|c|c|c|c|}
\hline \multirow{2}{*}{$\begin{array}{l}\text { NTS hole } \\
\text { number }\end{array}$} & \multirow{2}{*}{$\begin{array}{l}\text { USGS standard } \\
\text { Identification }\end{array}$} & \multirow{2}{*}{$\begin{array}{l}\text { Latitude } \\
\text { (degrees, } \\
\text { minutes, } \\
\text { seconds) }\end{array}$} & \multirow{2}{*}{$\begin{array}{l}\text { Longitude } \\
\text { (degrees, } \\
\text { minutes, } \\
\text { seconds) }\end{array}$} & \multirow{2}{*}{$\begin{array}{l}\text { Date hole } \\
\text { completed } \\
\text { (month, } \\
\text { day, year) }\end{array}$} & \multirow{2}{*}{$\begin{array}{l}\text { Land- } \\
\text { surface } \\
\text { altitude } \\
\text { (feet) }\end{array}$} & \multirow{2}{*}{$\begin{array}{l}\text { Hole } \\
\text { depth } \\
\text { (feet) }\end{array}$} & \multicolumn{3}{|c|}{$\begin{array}{c}\text { Depth of } \\
\text { open intervals }\end{array}$} & \multicolumn{4}{|c|}{ Measurement } \\
\hline & & & & & & & $\begin{array}{c}\text { Top } \\
\text { (feet) }\end{array}$ & $\begin{array}{c}\text { Bottom } \\
\text { (feet) }\end{array}$ & Type & $\begin{array}{c}\text { Date } \\
\text { (month, } \\
\text { day, year) }\end{array}$ & $\begin{array}{l}\text { Depth to } \\
\text { water } \\
\text { (feet) }\end{array}$ & Method & $\begin{array}{l}\text { Site } \\
\text { status }\end{array}$ \\
\hline UE-3e 4-2 & 370411116025911 & 370411 & 1160259 & $03-22-90$ & 4,082 & 1,919 & 1,887 & 1,908 & $S$ & $\begin{array}{l}07-03-91 \\
07-15-91 \\
07-31-91 \\
08-12-91 \\
08-21-91 \\
09-12-91\end{array}$ & $\begin{array}{l}1,286 \\
1,286 \\
1,287 \\
1,288 \\
1,288 \\
1,290\end{array}$ & $\begin{array}{l}\mathrm{V} \\
\mathrm{V} \\
\mathrm{V} \\
\mathrm{V} \\
\mathrm{V} \\
\mathrm{V}\end{array}$ & $\begin{array}{l}- \\
- \\
- \\
- \\
-\end{array}$ \\
\hline \multirow[t]{7}{*}{ UE-3e 4-3 } & 370411116025912 & 370411 & 1160259 & $03-26-90$ & 4,082 & 1,661 & 1,619 & 1,640 & $S$ & $\begin{array}{l}03-29-90 \\
03-30-90 \\
04-03-90 \\
05-09-90 \\
05-16-90\end{array}$ & $\begin{array}{l}1,551 \\
1,550 \\
1,551 \\
1,559 \\
1,554\end{array}$ & $\begin{array}{l}V \\
V \\
V \\
V \\
V\end{array}$ & $\begin{array}{l}\text { Z } \\
- \\
- \\
- \\
-\end{array}$ \\
\hline & & & & & & & & & & $\begin{array}{l}05-23-90 \\
05-30-90 \\
06-12-90 \\
06-20-90 \\
06-27-90\end{array}$ & $\begin{array}{l}1,552 \\
1,552 \\
1,558 \\
1,552 \\
1,552\end{array}$ & $\begin{array}{l}\mathrm{V} \\
\mathrm{V} \\
\mathrm{V} \\
\mathrm{V} \\
\mathrm{V}\end{array}$ & $\begin{array}{l}- \\
- \\
-\end{array}$ \\
\hline & & & & & & & & & & $\begin{array}{l}07-05-90 \\
07-11-90 \\
07-17-90 \\
07-20-90 \\
08-03-90\end{array}$ & $\begin{array}{l}1,552 \\
1,552 \\
1,552 \\
1,552 \\
1,560\end{array}$ & $\begin{array}{l}V \\
V \\
V \\
V \\
V\end{array}$ & $\begin{array}{l}- \\
- \\
- \\
\mathrm{R}\end{array}$ \\
\hline & & & & & & & & & & $\begin{array}{l}08-13-90 \\
08-17-90 \\
08-28-90 \\
09-12-90 \\
09-24-90\end{array}$ & $\begin{array}{l}1,554 \\
1,553 \\
1,552 \\
1,552 \\
1,553\end{array}$ & $\begin{array}{l}\mathrm{V} \\
\mathrm{V} \\
\mathrm{V} \\
\mathrm{V} \\
\mathrm{V}\end{array}$ & $\begin{array}{l}- \\
- \\
- \\
-\end{array}$ \\
\hline & & & & & & & & & & $\begin{array}{l}10-05-90 \\
10-23-90 \\
11-15-90 \\
11-30-90 \\
12-18-90\end{array}$ & $\begin{array}{l}1,553 \\
1,553 \\
1,552 \\
1,553 \\
1,555\end{array}$ & $\begin{array}{l}\mathrm{V} \\
\mathrm{V} \\
\mathrm{V} \\
\mathrm{V} \\
\mathrm{V}\end{array}$ & $\begin{array}{l}- \\
- \\
- \\
\text { R }\end{array}$ \\
\hline & & & & & & & & & & $\begin{array}{l}01-07-91 \\
01-22-91 \\
02-11-91 \\
02-25-91 \\
03-14-91\end{array}$ & $\begin{array}{l}1,553 \\
1,553 \\
1,553 \\
1,553 \\
1,552\end{array}$ & $\begin{array}{l}V \\
V \\
V \\
V \\
V\end{array}$ & $\begin{array}{l}- \\
- \\
- \\
-\end{array}$ \\
\hline & & & & & & & & & & $\begin{array}{l}03-25-91 \\
04-09-91 \\
04-22-91 \\
05-08-91 \\
05-21-91\end{array}$ & $\begin{array}{l}1,552 \\
1,552 \\
1,553 \\
1,552 \\
1,552\end{array}$ & $\begin{array}{l}\text { V } \\
V \\
V \\
V \\
V\end{array}$ & $\begin{array}{l}- \\
- \\
- \\
-\end{array}$ \\
\hline
\end{tabular}




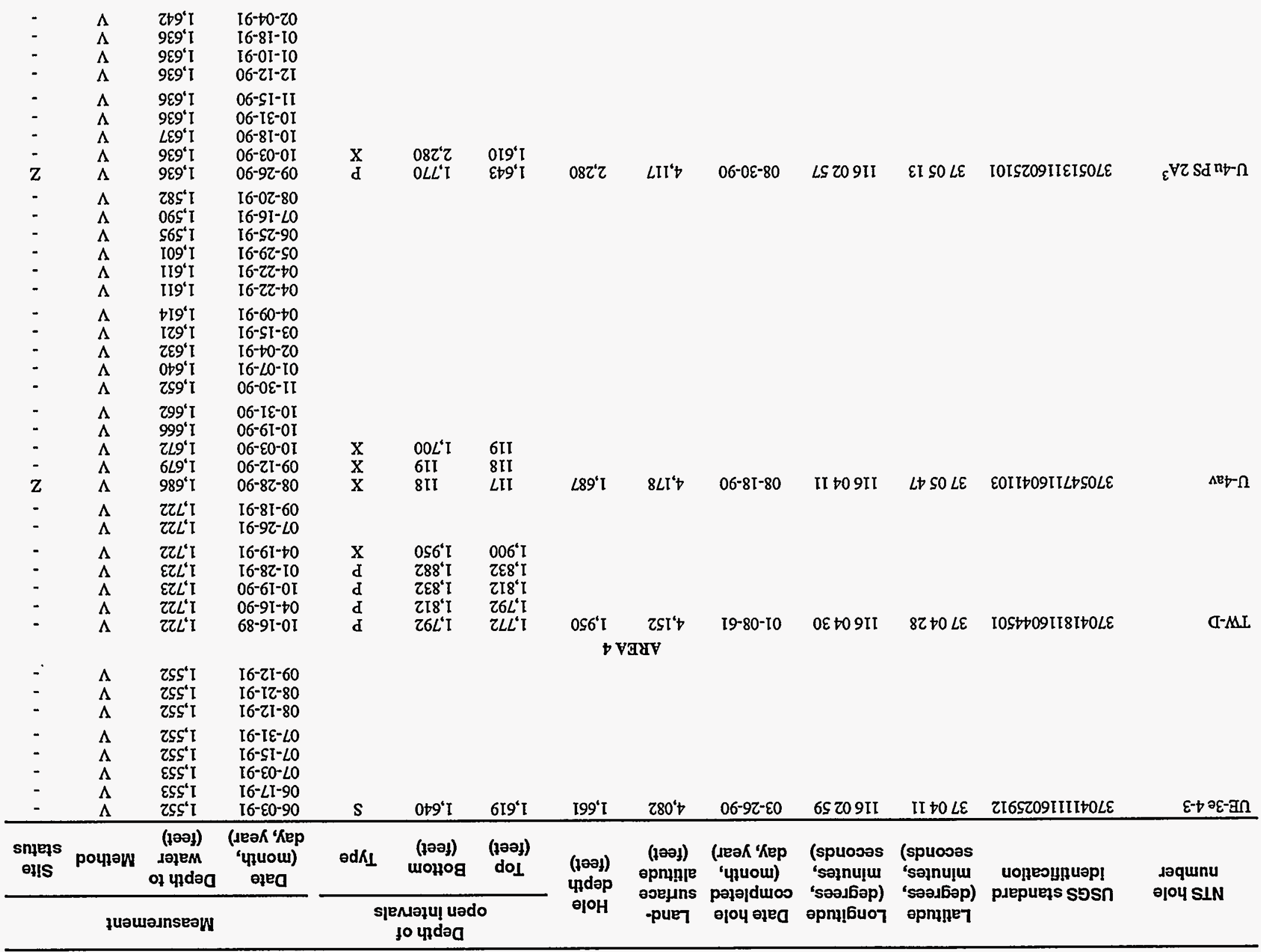


Table 1. Depth to water in wells and test holes at the Nevada Test Site (NTS) and the Faultless Site, Nye County, Nevada, water years 1990-91--Continued

\begin{tabular}{|c|c|c|c|c|c|c|c|c|c|c|c|c|c|}
\hline \multirow{2}{*}{$\begin{array}{l}\text { NTS hole } \\
\text { number }\end{array}$} & \multirow{2}{*}{$\begin{array}{l}\text { USGS standard } \\
\text { identification }\end{array}$} & \multirow{2}{*}{$\begin{array}{l}\text { Latitude } \\
\text { (degrees, } \\
\text { minutes, } \\
\text { seconds) }\end{array}$} & \multirow{2}{*}{$\begin{array}{l}\text { Longitude } \\
\text { (degrees, } \\
\text { minutes, } \\
\text { seconds) }\end{array}$} & \multirow{2}{*}{$\begin{array}{l}\text { Date hole } \\
\text { completed } \\
\text { (month, } \\
\text { day, year) }\end{array}$} & \multirow{2}{*}{$\begin{array}{l}\text { Land- } \\
\text { surface } \\
\text { altitude } \\
\text { (feet) }\end{array}$} & \multirow{2}{*}{$\begin{array}{l}\text { Hole } \\
\text { depth } \\
\text { (feet) }\end{array}$} & \multicolumn{3}{|c|}{$\begin{array}{c}\text { Depth of } \\
\text { open intervals }\end{array}$} & \multicolumn{4}{|c|}{ Measurement } \\
\hline & & & & & & & $\begin{array}{l}\text { Tóp } \\
\text { (feet) }\end{array}$ & $\begin{array}{l}\text { Bottom } \\
\text { (feet) }\end{array}$ & Type & $\begin{array}{c}\text { Date } \\
\text { (month, } \\
\text { day, year) }\end{array}$ & $\begin{array}{l}\text { Depth to } \\
\text { water } \\
\text { (feet) }\end{array}$ & Method & $\begin{array}{l}\text { Site } \\
\text { status }\end{array}$ \\
\hline \multirow[t]{2}{*}{$\mathrm{U}-4 \mathrm{u}$ PS $2 \mathrm{~A}^{3}$} & 370513116025101 & 370513 & 1160257 & $08-30-90$ & 4,117 & 2,280 & $\begin{array}{l}1,643 \\
1,610\end{array}$ & $\begin{array}{l}1,770 \\
2,280\end{array}$ & $\stackrel{P}{\mathrm{P}}$ & $\begin{array}{l}03-15-91 \\
04-09-91 \\
04-22-91 \\
05-21-91 \\
06-03-91\end{array}$ & $\begin{array}{l}1,642 \\
1,641 \\
1,641 \\
1,641 \\
1,641\end{array}$ & $\begin{array}{l}\mathrm{V} \\
\mathrm{V} \\
\mathrm{V} \\
\mathrm{V} \\
\mathrm{V}\end{array}$ & $\begin{array}{l}- \\
- \\
- \\
-\end{array}$ \\
\hline & & & & & & & & & & $\begin{array}{l}07-09-91 \\
08-12-91 \\
09-12-91\end{array}$ & $\begin{array}{l}1,641 \\
1,642 \\
1,641\end{array}$ & $\begin{array}{l}V \\
V \\
V\end{array}$ & $\dot{-}$ \\
\hline \multirow[t]{3}{*}{ UE-4av ${ }^{4}$} & 370547116041102 & 370547 & 1160411 & $07-13-89$ & 4,177 & 2,400 & $\begin{array}{l}1,720 \\
1,758\end{array}$ & $\begin{array}{l}1,758 \\
2,815\end{array}$ & $\begin{array}{l}\mathrm{X} \\
\mathrm{X}\end{array}$ & $\begin{array}{l}03-22-90 \\
05-01-90 \\
05-09-90 \\
05-17-90 \\
05-23-90\end{array}$ & $\begin{array}{l}1,627 \\
1,626 \\
1,626 \\
1,626 \\
1,626\end{array}$ & $\begin{array}{l}\mathrm{V} \\
\mathrm{V} \\
\mathrm{V} \\
\mathrm{V} \\
\mathrm{V}\end{array}$ & $\begin{array}{l}- \\
- \\
-\end{array}$ \\
\hline & & & & & & & & & & $\begin{array}{l}05-30-90 \\
06-06-90 \\
06-15-90\end{array}$ & $\begin{array}{l}1,626 \\
1,626 \\
1,626\end{array}$ & $\begin{array}{l}V \\
V \\
V\end{array}$ & : \\
\hline & & & & & & & & & & $\begin{array}{l}06-20-90 \\
06-27-90 \\
07-06-90 \\
07-11-90\end{array}$ & $\begin{array}{l}1,626 \\
1,626 \\
1,626 \\
1,626\end{array}$ & $\begin{array}{l}\text { V } \\
\text { V } \\
\text { V } \\
\text { V }\end{array}$ & $\begin{array}{l}- \\
- \\
-\end{array}$ \\
\hline $\mathrm{UE}-4 a v^{4}$ & 370547116041104 & 370547 & 1160411 & $07-27-90$ & 4,177 & 1,808 & $\begin{array}{r}116 \\
125 \\
1,720\end{array}$ & $\begin{array}{r}125 \\
1,582 \\
1,985\end{array}$ & $\begin{array}{l}X \\
X \\
X\end{array}$ & $\begin{array}{l}01-07-91 \\
02-04-91 \\
04-09-91 \\
06-12-91 \\
07-16-91 \\
08-20-91\end{array}$ & $\begin{array}{l}1,570 \\
1,571 \\
1,570 \\
1,560 \\
1,527 \\
1,540\end{array}$ & $\begin{array}{l}\text { V } \\
V \\
V \\
V \\
V \\
V\end{array}$ & $\begin{array}{l}Z \\
Z \\
Z \\
- \\
- \\
-\end{array}$ \\
\hline$U E-4 t^{5}$ & 370556116025404 & 370556 & 1160254 & $05-05-89$ & 4,144 & 1,721 & 1,619 & 1,721 & $x$ & $\begin{array}{l}01-24-90 \\
07-17-90 \\
08-01-90\end{array}$ & $\begin{array}{l}888 \\
876 \\
877\end{array}$ & $\begin{array}{l}\mathrm{V} \\
\mathrm{V} \\
\mathrm{V}\end{array}$ & $\begin{array}{l}\bar{Z} \\
\mathrm{R}\end{array}$ \\
\hline \multirow[t]{2}{*}{ UE-4t 1} & 370556116025405 & 370556 & 1160254 & $10-24-90$ & 4,144 & 2,010 & $\begin{array}{l}1,906 \\
1,963\end{array}$ & $\begin{array}{l}2,010 \\
1,992\end{array}$ & $\begin{array}{l}\mathrm{X} \\
\mathrm{P}\end{array}$ & $\begin{array}{l}11-02-90 \\
11-15-90 \\
11-30-90 \\
12-18-90 \\
01-07-91\end{array}$ & $\begin{array}{l}402 \\
384 \\
369 \\
353 \\
346\end{array}$ & $\begin{array}{l}V \\
V \\
V \\
V \\
V\end{array}$ & $\begin{array}{l}\text { Z } \\
- \\
- \\
- \\
-\end{array}$ \\
\hline & & & & & & & & & & $\begin{array}{l}01-22-91 \\
02-11-91 \\
02-25-91 \\
03-14-91 \\
03-25-91\end{array}$ & $\begin{array}{l}342 \\
333 \\
328 \\
323 \\
320\end{array}$ & $\begin{array}{l}V \\
V \\
V \\
V \\
V\end{array}$ & $\begin{array}{l}- \\
- \\
-\end{array}$ \\
\hline
\end{tabular}


Table 1. Depth to water in wells and test holes at the Nevada Test Site (NTS) and the Faultless Site, Nye County, Nevada, water years 1990-91-Continued

\begin{tabular}{|c|c|c|c|c|c|c|c|c|c|c|c|c|c|}
\hline \multirow{2}{*}{$\begin{array}{l}\text { NTS hole } \\
\text { number }\end{array}$} & \multirow{2}{*}{$\begin{array}{l}\text { USGS standard } \\
\text { Identification }\end{array}$} & \multirow{2}{*}{$\begin{array}{l}\text { Latitude } \\
\text { (degrees, } \\
\text { minutes, } \\
\text { seconds) }\end{array}$} & \multirow{2}{*}{$\begin{array}{l}\text { Longitude } \\
\text { (degrees, } \\
\text { minutes, } \\
\text { seconds) }\end{array}$} & \multirow{2}{*}{$\begin{array}{c}\text { Date hole } \\
\text { completed } \\
\text { (month, } \\
\text { day, year) }\end{array}$} & \multirow{2}{*}{$\begin{array}{l}\text { Land- } \\
\text { surface } \\
\text { altitude } \\
\text { (feet) }\end{array}$} & \multirow{2}{*}{$\begin{array}{l}\text { Hole } \\
\text { depth } \\
\text { (feet) }\end{array}$} & \multicolumn{3}{|c|}{$\begin{array}{c}\text { Depth of } \\
\text { open intervals }\end{array}$} & \multicolumn{4}{|c|}{ Measurement } \\
\hline & & & & & & & $\begin{array}{c}\text { Top } \\
\text { (feet) }\end{array}$ & $\begin{array}{c}\text { Bottom } \\
\text { (feet) }\end{array}$ & Type & $\begin{array}{c}\text { Date } \\
\text { (month, } \\
\text { day, year) }\end{array}$ & $\begin{array}{l}\text { Depth to } \\
\text { water } \\
\text { (feet) }\end{array}$ & Method & $\begin{array}{l}\text { Site } \\
\text { status }\end{array}$ \\
\hline \multirow[t]{3}{*}{ UE-4t 1} & 370556116025405 & 370556 & 1160254 & $10-24-90$ & 4,144 & 2,010 & $\begin{array}{l}1,906 \\
1,963\end{array}$ & $\begin{array}{l}2,010 \\
1,992\end{array}$ & $\begin{array}{l}\mathrm{X} \\
\mathrm{P}\end{array}$ & $\begin{array}{l}04-09-91 \\
04-22-91 \\
05-08-91 \\
05-20-91 \\
06-06-91\end{array}$ & $\begin{array}{l}318 \\
316 \\
313 \\
311 \\
309\end{array}$ & $\begin{array}{l}\mathrm{T} \\
\mathrm{T} \\
\mathrm{T} \\
\mathrm{T} \\
\mathrm{T}\end{array}$ & $\begin{array}{l}- \\
- \\
- \\
-\end{array}$ \\
\hline & & & & & & & & & & $\begin{array}{l}06-19-91 \\
07-05-91 \\
07-15-91 \\
07-29-91 \\
08-12-91\end{array}$ & $\begin{array}{l}309 \\
306 \\
305 \\
304 \\
303\end{array}$ & $\begin{array}{l}V \\
T \\
T \\
T \\
T\end{array}$ & $\begin{array}{l}- \\
- \\
- \\
-\end{array}$ \\
\hline & & & & & & & & & & $\begin{array}{l}08-28-91 \\
09-12-91\end{array}$ & $\begin{array}{l}302 \\
301\end{array}$ & $\begin{array}{l}\mathrm{T} \\
\mathrm{T}\end{array}$ & - \\
\hline \multirow[t]{6}{*}{ UE-4t 2} & 370556116025406 & 370556 & 1160254 & $10-24-90$ & 4,144 & 1,754 & $\begin{array}{l}1,564 \\
1,664\end{array}$ & $\begin{array}{l}1,754 \\
1,724\end{array}$ & $\begin{array}{l}\mathrm{X} \\
\mathrm{P}\end{array}$ & $\begin{array}{l}11-02-90 \\
11-15-90 \\
11-30-90 \\
12-18-90 \\
01-07-91\end{array}$ & $\begin{array}{l}361 \\
363 \\
365 \\
365 \\
371\end{array}$ & $\begin{array}{l}\text { V } \\
\text { V } \\
\text { V } \\
\text { V } \\
\text { V }\end{array}$ & $\begin{array}{l}Z \\
- \\
- \\
-\end{array}$ \\
\hline & & & & & & & & & & $\begin{array}{l}01-22-91 \\
02-11-91 \\
02-25-91 \\
03-14-91 \\
03-25-91\end{array}$ & $\begin{array}{l}372 \\
373 \\
374 \\
375 \\
375\end{array}$ & $\begin{array}{l}\text { V } \\
V \\
V \\
V \\
V\end{array}$ & $\begin{array}{l}- \\
- \\
-\end{array}$ \\
\hline & & & & & & & & & & $\begin{array}{l}04-09-91 \\
04-22-91 \\
05-08-91 \\
05-20-91 \\
06-06-91\end{array}$ & $\begin{array}{l}376 \\
376 \\
377 \\
378 \\
378\end{array}$ & $\begin{array}{l}T \\
T \\
T \\
T \\
T\end{array}$ & $\begin{array}{l}- \\
- \\
-\end{array}$ \\
\hline & & & & & & & & & & $\begin{array}{l}06-19-91 \\
06-19-91 \\
07-05-91 \\
07-15-91 \\
07-29-91\end{array}$ & $\begin{array}{l}379 \\
378 \\
382 \\
382 \\
382\end{array}$ & $\begin{array}{l}V \\
V \\
T \\
T \\
T\end{array}$ & $\begin{array}{l}- \\
- \\
-\end{array}$ \\
\hline & & & & & & & & & & $\begin{array}{l}08-12-91 \\
08-28-91 \\
09-12-91\end{array}$ & $\begin{array}{l}383 \\
383 \\
384\end{array}$ & $\begin{array}{l}\mathrm{T} \\
\mathrm{T} \\
\mathrm{T}\end{array}$ & : \\
\hline & \multicolumn{13}{|c|}{ AREA 5} \\
\hline RNM-2S & 364922115580101 & 364921 & 1155801 & $04-01-74$ & 3,133 & 1,120 & $\begin{array}{l}1,038 \\
1,120\end{array}$ & $\begin{array}{l}1,119 \\
1,156\end{array}$ & $\stackrel{p}{x}$ & $\begin{array}{l}09-27-90 \\
10-03-90 \\
10-12-90 \\
10-19-90 \\
10-26-90\end{array}$ & $\begin{array}{l}728 \\
728 \\
728 \\
728 \\
728\end{array}$ & $\begin{array}{l}V \\
V \\
V \\
V \\
V\end{array}$ & $\begin{array}{l}- \\
- \\
-\end{array}$ \\
\hline
\end{tabular}


Table 1. Depth to water in wells and test holes at the Nevada Test Site (NTS) and the Faultless Site, Nye County, Nevada, water years 1990-91--Continued

\begin{tabular}{|c|c|c|c|c|c|c|c|c|c|c|c|c|c|}
\hline \multirow{2}{*}{$\begin{array}{l}\text { NTS hole } \\
\text { number }\end{array}$} & \multirow{2}{*}{$\begin{array}{l}\text { USGS standard } \\
\text { identification }\end{array}$} & \multirow{2}{*}{$\begin{array}{r}\text { Latitude } \\
\text { (degrees, } \\
\text { minutes, } \\
\text { seconds) }\end{array}$} & \multirow{2}{*}{$\begin{array}{l}\text { Longitude } \\
\text { (degrees, } \\
\text { minutes, } \\
\text { seconds) }\end{array}$} & \multirow{2}{*}{$\begin{array}{l}\text { Date hole } \\
\text { completed } \\
\text { (month, } \\
\text { day, year) }\end{array}$} & \multirow{2}{*}{$\begin{array}{l}\text { Land- } \\
\text { surface } \\
\text { altitude } \\
\text { (feet) }\end{array}$} & \multirow{2}{*}{$\begin{array}{l}\text { Hole } \\
\text { depth } \\
\text { (feet) }\end{array}$} & \multicolumn{3}{|c|}{$\begin{array}{c}\text { Depth of } \\
\text { open intervals }\end{array}$} & \multicolumn{4}{|c|}{ Measurement } \\
\hline & & & & & & & $\begin{array}{l}\text { Top } \\
\text { (feet) }\end{array}$ & $\begin{array}{c}\text { Bottom } \\
\text { (feet) }\end{array}$ & Type & $\begin{array}{c}\text { Date } \\
\text { (month, } \\
\text { day, year) }\end{array}$ & $\begin{array}{l}\text { Depth to } \\
\text { water } \\
\text { (feet) }\end{array}$ & Method & $\begin{array}{l}\text { Site } \\
\text { status }\end{array}$ \\
\hline \multirow[t]{13}{*}{ RNM-2S } & 364922115580101 & 364921 & 1155801 & $04-01-74$ & 3,133 & 1,156 & 1,038 & 1,119 & $\frac{P}{x}$ & $11-02-90$ & 726 & $\begin{array}{l}T \\
\mathrm{~V}\end{array}$ & - \\
\hline & & & & & & & & & & $\begin{array}{l}11-15-90 \\
12-12-90\end{array}$ & $\begin{array}{r}726 \\
\ldots\end{array}$ & - & $\dot{\mathrm{p}}$ \\
\hline & & & & & & & & & & $01-10-91$ & 738 & $T$ & - \\
\hline & & & & & & & & & & 01-16-91 & 727 & $\frac{1}{4}$ & - \\
\hline & & & & & & & & & & $\begin{array}{l}11-24-91 \\
02-05-91\end{array}$ & 726 & T & $\overline{\mathrm{P}}$ \\
\hline & & & & & & & & & & $02-21-91$ & -- & - & $\mathrm{P}$ \\
\hline & & & & & & & & & & $\begin{array}{l}04-11-91 \\
04-26-91\end{array}$ & 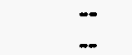 & - & $\begin{array}{l}\mathrm{p} \\
\mathrm{P}\end{array}$ \\
\hline & & & & & & & & & & $05-06-91$ & 728 & $\mathrm{~T}$ & $\mathbf{R}$ \\
\hline & & & & & & & & & & $05-17-91$ & -- & - & $P$ \\
\hline & & & & & & & & & & $\begin{array}{l}06-25-91 \\
06-91\end{array}$ & -- & : & $\begin{array}{l}\mathrm{P} \\
\mathrm{P}\end{array}$ \\
\hline & & & & & & & & & & 07-09-91 & -- & - & $\mathrm{P}$ \\
\hline & & & & & & & & & & 08-16-91 & -- & - & $P$ \\
\hline & & & & & & & & & & $\begin{array}{l}08-28-91 \\
09-27-91\end{array}$ & 727 & $\overline{\mathrm{T}}$ & $P$ \\
\hline UE-5f & 365213115564401 & 365213 & 1155644 & $06-14-65$ & 3,301 & 1,100 & 83 & 90 & $\mathrm{x}$ & $02-21-91$ & -- & $\mathrm{v}$ & 0 \\
\hline \multirow{14}{*}{ UE-5n } & 364915115574101 & 364915 & $11557.11 \mathrm{P}$ & 020176 & 3110 & 1687 & & 1,100 & $\begin{array}{c}x \\
p\end{array}$ & & & 8 & \\
\hline & 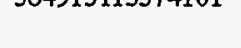 & 304710 & IAT & $200-05-10$ & $3,1 \delta 2$ & 1,001 & 1,523 & 1,687 & $\mathrm{x}$ & $\begin{array}{l}10-20-09 \\
12-15-89\end{array}$ & 705 & s & . \\
\hline & & & & & & & & & & $04-13-90$ & 705 & $\mathrm{~V}$ & - \\
\hline & & & & & & & & & & $\begin{array}{l}05-29-90 \\
10-26-90\end{array}$ & $\begin{array}{l}705 \\
705\end{array}$ & $\begin{array}{l}\mathrm{V} \\
\mathrm{S}\end{array}$ & $\overline{-}$ \\
\hline & & & & & & & & & & 01-10-91 & 705 & $\mathrm{~T}$ & - \\
\hline & & & & & & & & & & $02-21-91$ & 705 & $\mathrm{~V}$ & - \\
\hline & & & & & & & & & & $04-11-91$ & 705 & $\begin{array}{l}S \\
T\end{array}$ & :- \\
\hline & & & & & & & & & & $04-26-91$ & 705 & $\bar{T}$ & - \\
\hline & & & & & & & & & & 05-17-91 & 704 & $\mathrm{~T}$ & - \\
\hline & & & & & & & & & & $05-29-91$ & 704 & $T$ & - \\
\hline & & & & & & & & & & $\begin{array}{l}06-25-91 \\
07-09-91\end{array}$ & $\begin{array}{l}706 \\
704\end{array}$ & $T_{T}^{T}$ & $\cdot$ \\
\hline & & & & & & & & & & $07-19-91$ & 704 & s & . \\
\hline & & & & & & & & & & $\begin{array}{l}08-16-91 \\
08-28-91\end{array}$ & $\begin{array}{l}704 \\
704\end{array}$ & $\stackrel{T}{T}$ & - \\
\hline & & & & & & & & & & $09-27-91$ & 704 & $\mathrm{~T}$ & - \\
\hline WW-5b & 364805115580801 & 364805 & 1155808 & $05-07-51$ & 3,092 & 900 & 700 & 900 & $\mathrm{P}$ & $05-06-91$ & 689 & $\mathbf{T}$ & . \\
\hline WW-5c & 364708115574401 & 364720 & 1155749 & $03-24-54$ & 3,081 & 1,200 & 887 & 1,187 & $\mathbf{P}$ & $05-06-91$ & -- & - & $\mathbf{P}$ \\
\hline & & & & & & & 1,187 & 1,200 & $\mathrm{x}$ & & & & \\
\hline
\end{tabular}


Table 1. Depth to water in wells and test holes at the Nevada Test Site (NTS) and the Faultless Site, Nye County, Nevada, water years 1990-91--Continued

\begin{tabular}{|c|c|c|c|c|c|c|c|c|c|c|c|c|c|}
\hline \multirow{2}{*}{$\begin{array}{l}\text { NTS hole } \\
\text { number }\end{array}$} & \multirow{2}{*}{$\begin{array}{l}\text { USGS standard } \\
\text { identification }\end{array}$} & \multirow{2}{*}{$\begin{array}{c}\text { Latitude } \\
\text { (degrees, } \\
\text { minutes, } \\
\text { seconds) }\end{array}$} & \multirow{2}{*}{$\begin{array}{l}\text { Longitude } \\
\text { (degrees, } \\
\text { minutes, } \\
\text { seconds) }\end{array}$} & \multirow{2}{*}{$\begin{array}{l}\text { Date hole } \\
\text { completed } \\
\text { (month, } \\
\text { day, year) }\end{array}$} & \multirow{2}{*}{$\begin{array}{l}\text { Land- } \\
\text { surface } \\
\text { altitude } \\
\text { (feet) }\end{array}$} & \multirow{2}{*}{$\begin{array}{l}\text { Hole } \\
\text { depth } \\
\text { (feet) }\end{array}$} & \multicolumn{3}{|c|}{$\begin{array}{c}\text { Depth of } \\
\text { open intervals }\end{array}$} & \multicolumn{4}{|c|}{ Measurement } \\
\hline & & & & & & & $\begin{array}{l}\text { Top } \\
\text { (feet) }\end{array}$ & $\begin{array}{c}\text { Bottom } \\
\text { (feet) }\end{array}$ & Type & $\begin{array}{c}\text { Date } \\
\text { (month, } \\
\text { day, year) }\end{array}$ & $\begin{array}{c}\text { Depth to } \\
\text { water } \\
\text { (feet) }\end{array}$ & Method & $\begin{array}{l}\text { Site } \\
\text { status }\end{array}$ \\
\hline \multicolumn{14}{|c|}{ AREA 6} \\
\hline \multirow[t]{2}{*}{ TW-B } & 365849116002101 & 365845 & 1160049 & $05-14-61$ & 3,929 & 1,670 & $\begin{array}{l}1,432 \\
1,512\end{array}$ & $\begin{array}{l}1,452 \\
1,656\end{array}$ & $\begin{array}{l}\mathrm{P} \\
\mathrm{P}\end{array}$ & $\begin{array}{l}10-26-89 \\
12-15-89 \\
04-17-90 \\
11-05-90 \\
01-28-91\end{array}$ & $\begin{array}{l}1,504 \\
1,504 \\
1,504 \\
1,504 \\
1,504\end{array}$ & $\begin{array}{l}\text { V } \\
S \\
V \\
V \\
V\end{array}$ & $\begin{array}{l}- \\
- \\
-\end{array}$ \\
\hline & & & & & & & & & & $\begin{array}{l}03-06-91 \\
04-17-91 \\
07-18-91 \\
09-18-91\end{array}$ & $\begin{array}{l}1,504 \\
1,504 \\
1,504 \\
1,504\end{array}$ & $\begin{array}{l}S \\
V \\
S \\
V\end{array}$ & $\begin{array}{l}- \\
- \\
-\end{array}$ \\
\hline \multirow[t]{2}{*}{ UE-6d } & 365905116033201 & 365905 & 1160332 & $05-01-68$ & 3,947 & 3,864 & $\begin{array}{l}2,125 \\
2,617 \\
3,886\end{array}$ & $\begin{array}{l}2,617 \\
3,886 \\
3,896\end{array}$ & $\begin{array}{l}\mathrm{X} \\
\mathrm{X} \\
\mathrm{X}\end{array}$ & $\begin{array}{l}10-04-89 \\
01-18-90 \\
04-16-90 \\
11-05-90 \\
01-28-91\end{array}$ & $\begin{array}{l}1,516 \\
1,516 \\
1,515 \\
1,515 \\
1,515\end{array}$ & $\begin{array}{l}V \\
V \\
V \\
V \\
V\end{array}$ & $\begin{array}{l}- \\
- \\
-\end{array}$ \\
\hline & & & & & & & & & & $\begin{array}{l}04-17-91 \\
07-31-91 \\
09-18-91\end{array}$ & $\begin{array}{l}1,515 \\
1,515 \\
1,516\end{array}$ & $\begin{array}{l}\text { V } \\
V \\
V\end{array}$ & : \\
\hline UE-6e & 365905116012001 & 365905 & 1160120 & $11-01-73$ & 3,936 & 4,208 & $\begin{array}{l}2,090 \\
2,886\end{array}$ & $\begin{array}{l}2,886 \\
4,209\end{array}$ & $\begin{array}{l}\mathrm{x} \\
\mathrm{X}\end{array}$ & $\begin{array}{l}10-25-89 \\
04-16-90 \\
11-05-90 \\
04-17-91 \\
08-13-91 \\
09-18-91\end{array}$ & $\begin{array}{l}1,509 \\
1,508 \\
1,509 \\
1,507 \\
1,510 \\
1,510\end{array}$ & $\begin{array}{l}V \\
V \\
V \\
V \\
V \\
V\end{array}$ & $\begin{array}{l}- \\
- \\
- \\
-\end{array}$ \\
\hline \multirow[t]{4}{*}{ WW-4 } & 365418116012601 & 365418 & 1160126 & $11-18-81$ & 3,602 & 1,479 & $\begin{array}{r}942 \\
1,438\end{array}$ & $\begin{array}{l}1,436 \\
1,479\end{array}$ & $\stackrel{p}{x}$ & $\begin{array}{l}02-21-90 \\
02-22-90 \\
02-22-90 \\
02-23-90 \\
02-26-90\end{array}$ & $\begin{array}{l}836 \\
836 \\
835 \\
836 \\
835\end{array}$ & $\begin{array}{l}V \\
V \\
V \\
V \\
V\end{array}$ & $\begin{array}{l}- \\
- \\
\end{array}$ \\
\hline & & & & & & & & & & $\begin{array}{l}09-19-90 \\
11-05-90 \\
01-14-91 \\
02-19-91 \\
03-18-91\end{array}$ & $\begin{array}{r}835 \\
-- \\
835 \\
-- \\
--\end{array}$ & $\begin{array}{l}\mathrm{V} \\
\dot{\mathrm{V}} \\
-\end{array}$ & $\begin{array}{l}\mathrm{R} \\
\mathrm{P} \\
- \\
\mathrm{P} \\
\mathrm{P}\end{array}$ \\
\hline & & & & & & & & & & $\begin{array}{l}04-26-91 \\
05-06-91 \\
06-10-91 \\
07-05-91 \\
08-16-91\end{array}$ & $\begin{array}{r}- \\
- \\
- \\
835 \\
835\end{array}$ & $\begin{array}{l}- \\
- \\
\mathrm{T} \\
\mathrm{T}\end{array}$ & $\begin{array}{l}\mathrm{P} \\
\mathrm{P} \\
\mathrm{P} \\
- \\
-\end{array}$ \\
\hline & & & & & & & & & & $\begin{array}{l}08-28-91 \\
09-27-91\end{array}$ & $\begin{array}{l}-- \\
--\end{array}$ & - & $\begin{array}{l}\mathrm{P} \\
\mathrm{P}\end{array}$ \\
\hline
\end{tabular}


Table 1. Depth to water in wells and test holes at the Nevada Test Site (NTS) and the Faultiess Site, Nye County, Nevada, water years 1990-91--Continued

일

\begin{tabular}{|c|c|c|c|c|c|c|c|c|c|c|c|c|c|}
\hline \multirow{2}{*}{$\begin{array}{l}\text { NTS hole } \\
\text { number }\end{array}$} & \multirow{2}{*}{$\begin{array}{l}\text { USGS standard } \\
\text { Identification }\end{array}$} & \multirow{2}{*}{$\begin{array}{l}\text { Latitude } \\
\text { (degrees, } \\
\text { minutes, } \\
\text { seconds) }\end{array}$} & \multirow{2}{*}{$\begin{array}{l}\text { Longitude } \\
\text { (degrees, } \\
\text { minutes, } \\
\text { seconds) }\end{array}$} & \multirow{2}{*}{$\begin{array}{l}\text { Date hole } \\
\text { completed } \\
\text { (month, } \\
\text { day, year) }\end{array}$} & \multirow{2}{*}{$\begin{array}{l}\text { Land- } \\
\text { surface } \\
\text { altitude } \\
\text { (feet) }\end{array}$} & \multirow{2}{*}{$\begin{array}{l}\text { Hole } \\
\text { depth } \\
\text { (feet) }\end{array}$} & \multicolumn{3}{|c|}{$\begin{array}{c}\text { Depth of } \\
\text { open intervals }\end{array}$} & \multicolumn{4}{|c|}{ Measurement } \\
\hline & & & & & & & $\begin{array}{c}\text { Top } \\
\text { (feet) }\end{array}$ & $\begin{array}{c}\text { Bottom } \\
\text { (feet) }\end{array}$ & Type & $\begin{array}{c}\text { Date } \\
\text { (month, } \\
\text { day, year) }\end{array}$ & $\begin{array}{l}\text { Depth to } \\
\text { water } \\
\text { (feet) }\end{array}$ & Method & $\begin{array}{l}\text { Site } \\
\text { status }\end{array}$ \\
\hline \multirow[t]{4}{*}{$W W-4 a$} & 365412116013901 & 365412 & 1160139 & $02-21-90$ & 3,606 & 1,502 & $\begin{array}{l}1,066 \\
1,365 \\
1,501\end{array}$ & $\begin{array}{l}1,281 \\
1,457 \\
1,516\end{array}$ & $\begin{array}{l}P \\
P \\
X\end{array}$ & $\begin{array}{l}02-22-90 \\
02-23-90 \\
02-26-90 \\
05-29-90 \\
07-30-90\end{array}$ & $\begin{array}{r}836 \\
-- \\
835 \\
836 \\
836\end{array}$ & $\begin{array}{l}\mathrm{V} \\
\dot{-} \\
\mathrm{V} \\
\mathrm{V} \\
\mathrm{V}\end{array}$ & $\begin{array}{l}- \\
\mathrm{P} \\
- \\
-\end{array}$ \\
\hline & & & & & & & & & & $\begin{array}{l}10-26-90 \\
12-12-90 \\
01-14-91 \\
02-19-91 \\
03-18-91\end{array}$ & $\begin{array}{l}835 \\
835 \\
835 \\
836 \\
835\end{array}$ & $\begin{array}{l}\text { V } \\
V \\
V \\
V \\
V\end{array}$ & $\begin{array}{l}- \\
\dot{5} \\
-\end{array}$ \\
\hline & & & & & & & & & & $\begin{array}{l}04-26-91 \\
05-06-91 \\
06-10-91 \\
07-05-91 \\
08-16-91 \\
08-23-91\end{array}$ & $\begin{array}{l}836 \\
835 \\
835 \\
836 \\
835 \\
835\end{array}$ & $\begin{array}{l}\mathrm{T} \\
\mathrm{T} \\
\mathrm{T} \\
\mathrm{T} \\
\mathrm{T} \\
\mathrm{T}\end{array}$ & $\begin{array}{l}- \\
- \\
- \\
- \\
-\end{array}$ \\
\hline & \multicolumn{13}{|c|}{ AREA 7} \\
\hline $\mathrm{U}-7 \mathrm{cb}$ & 370514116000601 & 370514 & 1160006 & $02-14-86$ & 4,286 & 1,796 & $\begin{array}{l}299 \\
310\end{array}$ & $\begin{array}{r}310 \\
1,850\end{array}$ & $\begin{array}{l}X \\
X\end{array}$ & $\begin{array}{l}10-16-89 \\
03-07-90 \\
03-12-90 \\
03-27-90 \\
04-17-90\end{array}$ & $\begin{array}{l}1,784 \\
1,785 \\
1,786 \\
1,785 \\
1,785\end{array}$ & $\begin{array}{l}\mathrm{V} \\
\mathrm{S} \\
\mathrm{R} \\
\mathrm{S} \\
\mathrm{V}\end{array}$ & $\begin{array}{l}- \\
- \\
-\end{array}$ \\
\hline & & & & & & & & . & & $\begin{array}{l}05-10-90 \\
06-15-90 \\
06-28-90 \\
10-02-90\end{array}$ & $\begin{array}{l}1,785 \\
1,784 \\
1,785 \\
1,782\end{array}$ & $\begin{array}{l}\mathrm{V} \\
\mathrm{R} \\
\mathrm{S} \\
\mathrm{R}\end{array}$ & $\begin{array}{l}- \\
- \\
-\end{array}$ \\
\hline & & & & & & & & & & $\begin{array}{l}11-17-90 \\
12-05-90 \\
01-15-91 \\
01-18-91 \\
02-27-91\end{array}$ & $\begin{array}{l}1,784 \\
1,784 \\
1,784 \\
1,786 \\
1,786\end{array}$ & $\begin{array}{l}R \\
R \\
R \\
V \\
V\end{array}$ & : \\
\hline \multirow[t]{2}{*}{$\mathrm{UE}-7 \mathrm{nS}^{1}$} & 370556116000901 & 370556 & 1160009 & $07-14-76$ & 4,370 & 2,205 & $\begin{array}{l}1,995 \\
2,199 \\
1,960 \\
1,962\end{array}$ & $\begin{array}{l}2,199 \\
2,205 \\
2,020 \\
2,022\end{array}$ & $\begin{array}{l}P \\
X \\
P \\
P\end{array}$ & $\begin{array}{l}10-25-89 \\
11-08-89 \\
11-14-89 \\
11-16-89 \\
11-17-89\end{array}$ & $\begin{array}{l}1,969 \\
1,969 \\
1,969 \\
1,969 \\
1,969\end{array}$ & $\begin{array}{l}\text { V } \\
V \\
V \\
V \\
V\end{array}$ & $\begin{array}{l}- \\
- \\
-\end{array}$ \\
\hline & & & & & & & & & & $\begin{array}{l}11-28-89 \\
12-05-89 \\
12-06-89 \\
12-12-89 \\
01-09-90\end{array}$ & $\begin{array}{l}1,970 \\
1,969 \\
1,969 \\
1,970 \\
1,969\end{array}$ & $\begin{array}{l}\text { V } \\
\text { V } \\
\text { V } \\
\text { V } \\
\text { V }\end{array}$ & $\begin{array}{l}- \\
- \\
-\end{array}$ \\
\hline
\end{tabular}


Table 1. Depth to water in wells and test holes at the Nevada Test Site (NTS) and the Faultless Site, Nye County, Nevada, water years 1990-91--Continued

\begin{tabular}{|c|c|c|c|c|c|c|c|c|c|c|c|c|c|}
\hline \multirow{2}{*}{$\begin{array}{l}\text { NTS hole } \\
\text { number }\end{array}$} & \multirow{2}{*}{$\begin{array}{l}\text { USGS standard } \\
\text { identification }\end{array}$} & \multirow{2}{*}{$\begin{array}{c}\text { Latitude } \\
\text { (degrees, } \\
\text { minutes, } \\
\text { seconds) }\end{array}$} & \multirow{2}{*}{$\begin{array}{l}\text { Longitude } \\
\text { (degrees, } \\
\text { minutes, } \\
\text { seconds) }\end{array}$} & \multirow{2}{*}{$\begin{array}{l}\text { Date hole } \\
\text { completed } \\
\text { (month, } \\
\text { day, year) }\end{array}$} & \multirow{2}{*}{$\begin{array}{l}\text { Land- } \\
\text { surface } \\
\text { altitude } \\
\text { (feet) }\end{array}$} & \multirow{2}{*}{$\begin{array}{l}\text { Hole } \\
\text { depth } \\
\text { (feet) }\end{array}$} & \multicolumn{3}{|c|}{$\begin{array}{c}\text { Depth of } \\
\text { open intervals }\end{array}$} & \multicolumn{4}{|c|}{ Measurement } \\
\hline & & & & & & & $\begin{array}{c}\text { Top } \\
\text { (feet) }\end{array}$ & $\begin{array}{c}\text { Bottom } \\
\text { (feet) }\end{array}$ & Type & $\begin{array}{c}\text { Date } \\
\text { (month, } \\
\text { day, year) }\end{array}$ & $\begin{array}{l}\text { Depth to } \\
\text { water } \\
\text { (feet) }\end{array}$ & Method & $\begin{array}{l}\text { Site } \\
\text { status }\end{array}$ \\
\hline \multirow[t]{8}{*}{ UE-7nS } & 370556116000901 & 370556 & 1160009 & $07-14-76$ & 4,370 & 2,205 & $\begin{array}{l}1,995 \\
2,199 \\
1,960 \\
1,962\end{array}$ & $\begin{array}{l}2,199 \\
2,205 \\
2,020 \\
2,022\end{array}$ & $\begin{array}{l}\mathrm{P} \\
\mathrm{X} \\
\mathrm{P} \\
\mathrm{P}\end{array}$ & $\begin{array}{l}01-23-90 \\
01-24-90 \\
01-25-90 \\
01-31-90 \\
02-02-90\end{array}$ & $\begin{array}{l}1,969 \\
1,969 \\
1,969 \\
1,968 \\
1,969\end{array}$ & $\begin{array}{l}\mathrm{V} \\
\mathrm{V} \\
\mathrm{V} \\
\mathrm{V} \\
\mathrm{V}\end{array}$ & $\begin{array}{l}- \\
- \\
- \\
-\end{array}$ \\
\hline & & & & & & & & & & $\begin{array}{l}05-01-90 \\
05-09-90 \\
05-17-90 \\
05-23-90 \\
05-30-90\end{array}$ & $\begin{array}{l}1,969 \\
1,969 \\
1,968 \\
1,968 \\
1,968\end{array}$ & $\begin{array}{l}\mathrm{V} \\
\mathrm{V} \\
\mathrm{V} \\
\mathrm{V} \\
\mathrm{V}\end{array}$ & $\begin{array}{l}- \\
- \\
-\end{array}$ \\
\hline & & & & & & & & & & $\begin{array}{l}06-06-90 \\
06-15-90 \\
06-20-90 \\
06-27-90 \\
07-06-90\end{array}$ & $\begin{array}{l}1,969 \\
1,968 \\
1,969 \\
1,969 \\
1,969\end{array}$ & $\begin{array}{l}V \\
V \\
V \\
V \\
V\end{array}$ & $\begin{array}{l}- \\
- \\
- \\
-\end{array}$ \\
\hline & & & & & & & & & & $\begin{array}{l}07-11-90 \\
07-20-90 \\
07-30-90 \\
08-14-90 \\
08-27-90\end{array}$ & $\begin{array}{l}1,969 \\
1,969 \\
1,969 \\
1,969 \\
1,969\end{array}$ & $\begin{array}{l}V \\
V \\
V \\
V \\
V\end{array}$ & $\begin{array}{l}- \\
- \\
-\end{array}$ \\
\hline & & & & & & & & & & $\begin{array}{l}09-14-90 \\
09-20-90 \\
09-20-90 \\
09-21-90 \\
09-24-90\end{array}$ & $\begin{array}{l}1,969 \\
1,969 \\
1,970 \\
1,970 \\
1,970\end{array}$ & $\begin{array}{l}\text { V } \\
V \\
V \\
V \\
V\end{array}$ & $\begin{array}{l}- \\
- \\
-\end{array}$ \\
\hline & & & & & & & & & & $\begin{array}{l}10-05-90 \\
10-11-90 \\
10-16-90 \\
10-24-90 \\
10-30-90\end{array}$ & $\begin{array}{l}1,970 \\
1,970 \\
1,969 \\
1,969 \\
1,969\end{array}$ & $\begin{array}{l}V \\
V \\
V \\
S \\
V\end{array}$ & $\begin{array}{l}- \\
- \\
-\end{array}$ \\
\hline & & & & & & & & & & $\begin{array}{l}11-16-90 \\
11-30-90 \\
12-13-90 \\
12-18-90 \\
01-09-91\end{array}$ & $\begin{array}{l}1,970 \\
1,969 \\
1,969 \\
1,969 \\
1,969\end{array}$ & $\begin{array}{l}V \\
V \\
V \\
V \\
V\end{array}$ & $\begin{array}{l}- \\
- \\
- \\
-\end{array}$ \\
\hline & & & & & & & & & & $\begin{array}{l}01-17-91 \\
02-11-91 \\
02-26-91 \\
03-06-91 \\
03-13-91\end{array}$ & $\begin{array}{l}1,970 \\
1,969 \\
1,969 \\
1,969 \\
1,968\end{array}$ & $\begin{array}{l}\text { V } \\
\text { V } \\
S \\
S \\
\text { V }\end{array}$ & $\begin{array}{l}- \\
- \\
- \\
-\end{array}$ \\
\hline
\end{tabular}


Table 1. Depth to water in wells and test holes at the Nevada Test Site (NTS) and the Faultless Site, Nye County, Nevada, water years 1990-91--Continued

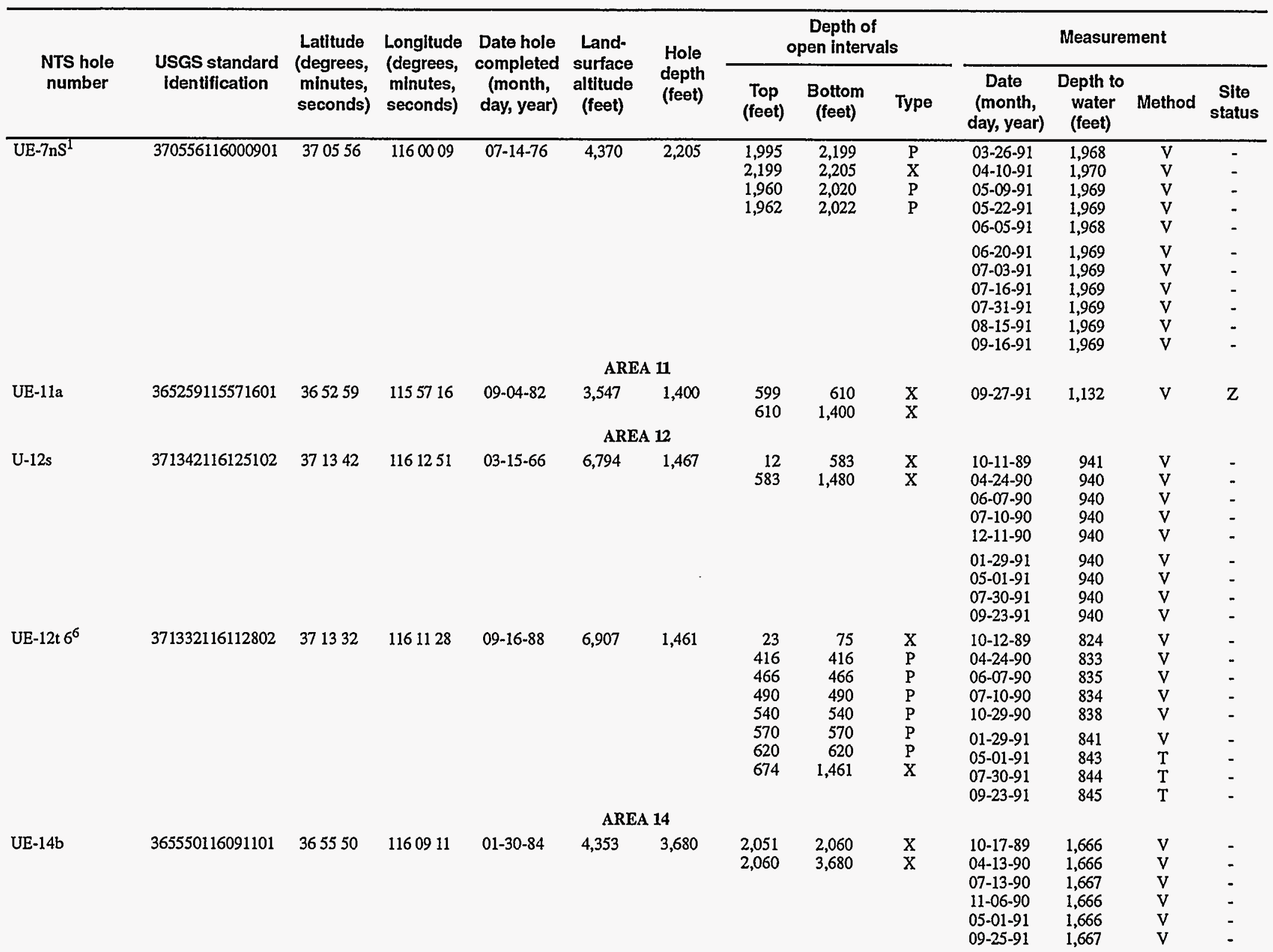


Table 1. Depth to water in wells and test holes at the Nevada Test Site (NTS) and the Faultless Site, Nye County, Nevada, water years 1990-91--Continued

\begin{tabular}{|c|c|c|c|c|c|c|c|c|c|c|c|c|c|}
\hline \multirow[b]{2}{*}{$\begin{array}{l}\text { NTS hole } \\
\text { number }\end{array}$} & \multirow[b]{2}{*}{$\begin{array}{l}\text { USGS standard } \\
\text { identification }\end{array}$} & \multirow{2}{*}{$\begin{array}{c}\text { Latitude } \\
\text { (degrees, } \\
\text { minutes, } \\
\text { seconds) }\end{array}$} & \multirow{2}{*}{$\begin{array}{l}\text { Longitude } \\
\text { (degrees, } \\
\text { minutes, } \\
\text { sedonds) }\end{array}$} & \multirow{2}{*}{$\begin{array}{l}\text { Date hole } \\
\text { completed } \\
\text { (month, } \\
\text { day, year) }\end{array}$} & \multirow{2}{*}{$\begin{array}{l}\text { Land- } \\
\text { surface } \\
\text { altitude } \\
\text { (feet) }\end{array}$} & \multirow{2}{*}{$\begin{array}{l}\text { Hole } \\
\text { depth } \\
\text { (feet) }\end{array}$} & \multicolumn{3}{|c|}{$\begin{array}{c}\text { Depth of } \\
\text { open intervals }\end{array}$} & \multicolumn{4}{|c|}{ Measurement } \\
\hline & & & & & & & $\begin{array}{c}\text { Top } \\
\text { (feet) }\end{array}$ & $\begin{array}{c}\text { Bottom } \\
\text { (feet) }\end{array}$ & Type & $\begin{array}{c}\text { Date } \\
\text { (month, } \\
\text { day, year) }\end{array}$ & $\begin{array}{l}\text { Depth to } \\
\text { water } \\
\text { (feet) }\end{array}$ & Method & $\begin{array}{c}\text { Site } \\
\text { status }\end{array}$ \\
\hline \multicolumn{14}{|c|}{ AREA 16} \\
\hline UE-16f & $\begin{array}{c}370208116092402 \\
.\end{array}$ & 370208 & 1160924 & $09-23-77$ & 4,652 & 1,409 & 1,293 & 1,479 & $\mathrm{x}$ & $\begin{array}{l}10-17-89 \\
04-13-90 \\
11-07-90 \\
04-18-91 \\
08-28-91 \\
09-25-91\end{array}$ & $\begin{array}{l}367 \\
367 \\
367 \\
367 \\
367 \\
367\end{array}$ & $\begin{array}{l}V \\
V \\
V \\
T \\
T \\
T\end{array}$ & $\begin{array}{l}- \\
- \\
- \\
-\end{array}$ \\
\hline \multicolumn{14}{|c|}{ AREA 17} \\
\hline TW-1 & 370929116132311 & 370929 & 1161323 & --80 & 6,156 & 3,694 & $\begin{array}{l}1,910 \\
2,030 \\
2,100 \\
2,230 \\
2,370\end{array}$ & $\begin{array}{l}1,950 \\
2,050 \\
2,160 \\
2,270 \\
2,430\end{array}$ & $\begin{array}{l}P \\
P \\
P \\
P \\
P\end{array}$ & $\begin{array}{l}10-04-89 \\
05-08-90 \\
07-10-90 \\
10-29-90 \\
02-12-91\end{array}$ & $\begin{array}{l}1,466 \\
1,465 \\
1,465 \\
1,465 \\
1,465\end{array}$ & $\begin{array}{l}\mathrm{V} \\
\mathrm{V} \\
\mathrm{V} \\
\mathrm{V} \\
\mathrm{V}\end{array}$ & $\begin{array}{l}- \\
- \\
-\end{array}$ \\
\hline & & & & & & & & & & $\begin{array}{l}05-01-91 \\
07-30-91 \\
09-23-91\end{array}$ & $\begin{array}{l}1,464 \\
1,465 \\
1,465\end{array}$ & $\begin{array}{l}V \\
V \\
V\end{array}$ & 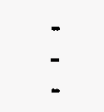 \\
\hline UE-17a & 370425116095801 & 370425 & 1160958 & $09-23-76$ & 4,696 & 1,207 & $\begin{array}{r}745 \\
1,005 \\
1,065 \\
1,210\end{array}$ & $\begin{array}{r}825 \\
1,015 \\
1,190 \\
1,214\end{array}$ & $\begin{array}{l}\mathrm{P} \\
\mathrm{P} \\
\mathrm{P} \\
\mathrm{X}\end{array}$ & $\begin{array}{l}10-04-89 \\
04-13-90 \\
09-13-90 \\
05-01-91 \\
09-23-91\end{array}$ & $\begin{array}{l}638 \\
637 \\
637 \\
637 \\
637\end{array}$ & $\begin{array}{l}V \\
V \\
V \\
T \\
T\end{array}$ & $\begin{array}{l}S \\
S \\
S \\
S \\
-\end{array}$ \\
\hline \multicolumn{14}{|c|}{ AREA 18} \\
\hline UE-18r & 370806116264001 & 370805 & 1162641 & $01-24-68$ & 5,538 & 4,930 & $\begin{array}{l}1,629 \\
1,632 \\
4,988\end{array}$ & $\begin{array}{l}1,632 \\
4,988 \\
5,004\end{array}$ & $\begin{array}{l}\mathrm{X} \\
\mathrm{X} \\
\mathrm{X}\end{array}$ & $\begin{array}{l}10-12-89 \\
04-12-90 \\
11-06-90 \\
04-24-91 \\
09-25-91\end{array}$ & $\begin{array}{l}1,365 \\
1,365 \\
1,365 \\
1,365 \\
1,365\end{array}$ & $\begin{array}{l}V \\
V \\
V \\
V \\
V\end{array}$ & $\begin{array}{l}- \\
- \\
-\end{array}$ \\
\hline UE-18t & 370741116194501 & 370741 & 1161945 & $10-05-78$ & 5,201 & 2,600 & 1,896 & 2,600 & $\mathrm{x}$ & $\begin{array}{l}10-12-89 \\
04-12-90 \\
11-06-90 \\
04-24-91 \\
09-25-91\end{array}$ & $\begin{array}{l}916 \\
915 \\
916 \\
915 \\
915\end{array}$ & $\begin{array}{l}\mathrm{V} \\
\mathrm{V} \\
\mathrm{V} \\
\mathrm{V} \\
\mathrm{V}\end{array}$ & $\begin{array}{l}- \\
- \\
-\end{array}$ \\
\hline \multicolumn{14}{|c|}{ AREA 19} \\
\hline U-19av & 371519116223301 & 371519 & 1162233 & $04-03-89$ & 6,514 & 1,000 & $\begin{array}{l}52 \\
56\end{array}$ & $\begin{array}{r}56 \\
2,144\end{array}$ & $\begin{array}{l}\mathrm{X} \\
\mathrm{X}\end{array}$ & $11-29-90$ & -- & $\mathrm{V}$ & D \\
\hline U-19az & 371339116221601 & 371339 & 1162216 & $12-05-88$ & 6,753 & 2,130 & $\begin{array}{l}77 \\
79\end{array}$ & $\begin{array}{r}79 \\
2,130\end{array}$ & $\begin{array}{l}\mathrm{X} \\
\mathrm{X}\end{array}$ & $\begin{array}{l}10-11-89 \\
04-11-90 \\
07-02-90\end{array}$ & $\begin{array}{l}2,079 \\
2,079 \\
2,079\end{array}$ & $\begin{array}{l}\mathrm{V} \\
\mathrm{V} \\
\mathrm{V}\end{array}$ & : \\
\hline
\end{tabular}


Table 1. Depth to water in wells and test holes at the Nevada Test Site (NTS) and the Faultless Site, Nye County, Nevada, water years 1990-91--Continued

\begin{tabular}{|c|c|c|c|c|c|c|c|c|c|c|c|c|c|}
\hline \multirow{2}{*}{$\begin{array}{l}\text { NTS hole } \\
\text { number }\end{array}$} & \multirow{2}{*}{$\begin{array}{l}\text { USGS standard } \\
\text { identification }\end{array}$} & \multirow{2}{*}{$\begin{array}{l}\text { Latltude } \\
\text { (degrees, } \\
\text { minutes, } \\
\text { seconds) }\end{array}$} & \multirow{2}{*}{$\begin{array}{l}\text { Longitude } \\
\text { (degrees, } \\
\text { minutes, } \\
\text { seconds) }\end{array}$} & \multirow{2}{*}{$\begin{array}{l}\text { Date hole } \\
\text { completed } \\
\text { (month, } \\
\text { day, year) }\end{array}$} & \multirow{2}{*}{$\begin{array}{l}\text { Land- } \\
\text { surface } \\
\text { altitude } \\
\text { (feet) }\end{array}$} & \multirow{2}{*}{$\begin{array}{l}\text { Hole } \\
\text { depth } \\
\text { (feet) }\end{array}$} & \multicolumn{3}{|c|}{$\begin{array}{c}\text { Depth of } \\
\text { open Intervals }\end{array}$} & \multicolumn{4}{|c|}{ Measurement } \\
\hline & & & & & & & $\begin{array}{c}\text { Top } \\
\text { (feet) }\end{array}$ & $\begin{array}{l}\text { Bottom } \\
\text { (feet) }\end{array}$ & Type & $\begin{array}{c}\text { Date } \\
\text { (month, } \\
\text { day, year) }\end{array}$ & $\begin{array}{l}\text { Depth to } \\
\text { water } \\
\text { (feet) }\end{array}$ & Method & $\begin{array}{l}\text { Site } \\
\text { status }\end{array}$ \\
\hline \multirow[t]{5}{*}{ U-19ba } & 371746116184601 & 371746 & 1161846 & $09-11-89$ & 7,037 & 2,177 & $\begin{array}{l}69 \\
70\end{array}$ & $\begin{array}{r}70 \\
2,177\end{array}$ & $\begin{array}{l}\mathrm{X} \\
\mathrm{X}\end{array}$ & $\begin{array}{l}10-02-89 \\
10-10-89 \\
10-17-89 \\
10-23-89 \\
10-30-89\end{array}$ & $\begin{array}{l}2,160 \\
2,159 \\
2,159 \\
2,158 \\
2,158\end{array}$ & $\begin{array}{l}\mathrm{V} \\
\mathrm{V} \\
\mathrm{V} \\
\mathrm{V} \\
\mathrm{V}\end{array}$ & $\begin{array}{l}Z \\
- \\
- \\
-\end{array}$ \\
\hline & & & & & & & & & & $\begin{array}{l}11-09-89 \\
11-13-89 \\
11-27-89 \\
11-29-89 \\
12-04-89\end{array}$ & $\begin{array}{l}2,157 \\
2,157 \\
2,157 \\
2,157 \\
2,156\end{array}$ & $\begin{array}{l}\text { V } \\
V \\
V \\
V \\
V\end{array}$ & $\begin{array}{l}- \\
- \\
- \\
-\end{array}$ \\
\hline & & & & & & & & & & $\begin{array}{l}01-22-90 \\
02-13-90 \\
02-26-90 \\
03-08-90 \\
04-09-90\end{array}$ & $\begin{array}{l}2,154 \\
2,155 \\
2,154 \\
2,153 \\
2,153\end{array}$ & $\begin{array}{l}\text { V } \\
V \\
V \\
V \\
V\end{array}$ & $\begin{array}{l}- \\
- \\
-\end{array}$ \\
\hline & & & & & & & & & & $\begin{array}{l}05-07-90 \\
05-14-90 \\
05-22-90 \\
05-31-90 \\
06-05-90\end{array}$ & $\begin{array}{l}2,153 \\
2,153 \\
2,153 \\
2,153 \\
2,153\end{array}$ & $\begin{array}{l}\mathrm{V} \\
\mathrm{V} \\
\mathrm{V} \\
\mathrm{V} \\
\mathrm{V}\end{array}$ & $\begin{array}{l}- \\
- \\
- \\
-\end{array}$ \\
\hline & & & & & & & & & & $\begin{array}{l}06-25-90 \\
07-03-90 \\
08-02-90 \\
12-11-90\end{array}$ & $\begin{array}{l}2,153 \\
2,153 \\
2,153 \\
2,152\end{array}$ & $\begin{array}{l}\mathrm{V} \\
\mathrm{V} \\
\mathrm{V} \\
\mathrm{V}\end{array}$ & $\begin{array}{l}- \\
- \\
-\end{array}$ \\
\hline U-19ba 1 & 371746116184701 & 371746 & 1161847 & $09-17-90$ & 7,038 & 2,183 & 65 & 2,340 & $\mathrm{X}$ & $\begin{array}{l}10-10-90 \\
12-11-90\end{array}$ & - & $\begin{array}{l}\mathrm{V} \\
\mathrm{V}\end{array}$ & $\begin{array}{l}\mathrm{D} \\
\mathrm{D}\end{array}$ \\
\hline U-19ba 2 & 371745116184701 & 371746 & 1161847 & $09-26-90$ & 7,039 & 2,213 & $\begin{array}{l}64 \\
67\end{array}$ & $\begin{array}{r}67 \\
2,340\end{array}$ & $\begin{array}{l}\mathrm{X} \\
\mathrm{X}\end{array}$ & $10-02-90$ & - & V & D \\
\hline U-19ba 3 & 371746116184702 & 371747 & 1161847 & $11-28-90$ & 7,037 & 2,223 & 60 & 2,340 & $\mathrm{X}$ & $12-11-90$ & -- & $\mathrm{V}$ & $\mathrm{D}$ \\
\hline U-19bf & 372046116182701 & 372046 & 1161827 & $01-11-90$ & 6,829 & 527 & 60 & 1,546 & $X$ & $11-29-90$ & - & V & 0 \\
\hline U-19bg & 371621116213501 & 371621 & 1162135 & $04-20-90$ & 6,691 & 2,109 & 72 & 2,157 & $\mathrm{X}$ & $\begin{array}{l}06-26-90 \\
08-16-90 \\
06-06-91\end{array}$ & $\begin{array}{l}-- \\
- \\
-\end{array}$ & $\begin{array}{l}\text { V } \\
\text { V } \\
\text { V }\end{array}$ & $\begin{array}{l}D \\
D \\
D\end{array}$ \\
\hline U-19bg 1 & 371620116213501 & 371620 & 1162135 & $07-15-91$ & 6,694 & 2,218 & $\begin{array}{l}74 \\
75\end{array}$ & $\begin{array}{r}75 \\
2,250\end{array}$ & $\begin{array}{l}\mathrm{X} \\
\mathrm{X}\end{array}$ & $\begin{array}{l}08-20-91 \\
09-09-91 \\
09-24-91\end{array}$ & $\begin{array}{l}2,119 \\
2,119 \\
2,119\end{array}$ & $\begin{array}{l}V \\
V \\
V\end{array}$ & $\begin{array}{l}Z \\
- \\
-\end{array}$ \\
\hline \multirow[t]{2}{*}{ U-19bh } & 371349116222001 & 371349 & 1162220 & $06-14-91$ & 6,768 & 2,148 & $\begin{array}{l}70 \\
72\end{array}$ & $\begin{array}{r}72 \\
2,148\end{array}$ & $\begin{array}{l}\mathrm{X} \\
\mathrm{X}\end{array}$ & $\begin{array}{l}06-24-91 \\
07-02-91 \\
07-08-91 \\
07-26-91 \\
08-14-91\end{array}$ & $\begin{array}{l}2,140 \\
2,135 \\
2,131 \\
2,122 \\
2,116\end{array}$ & $\begin{array}{l}V \\
V \\
V \\
V \\
V\end{array}$ & $\begin{array}{l}\text { Z } \\
- \\
- \\
- \\
-\end{array}$ \\
\hline & & & & & & & & & & $\begin{array}{l}09-09-91 \\
09-24-91\end{array}$ & $\begin{array}{l}2,108 \\
2,105\end{array}$ & $\begin{array}{l}\mathrm{V} \\
\mathrm{V}\end{array}$ & - \\
\hline
\end{tabular}




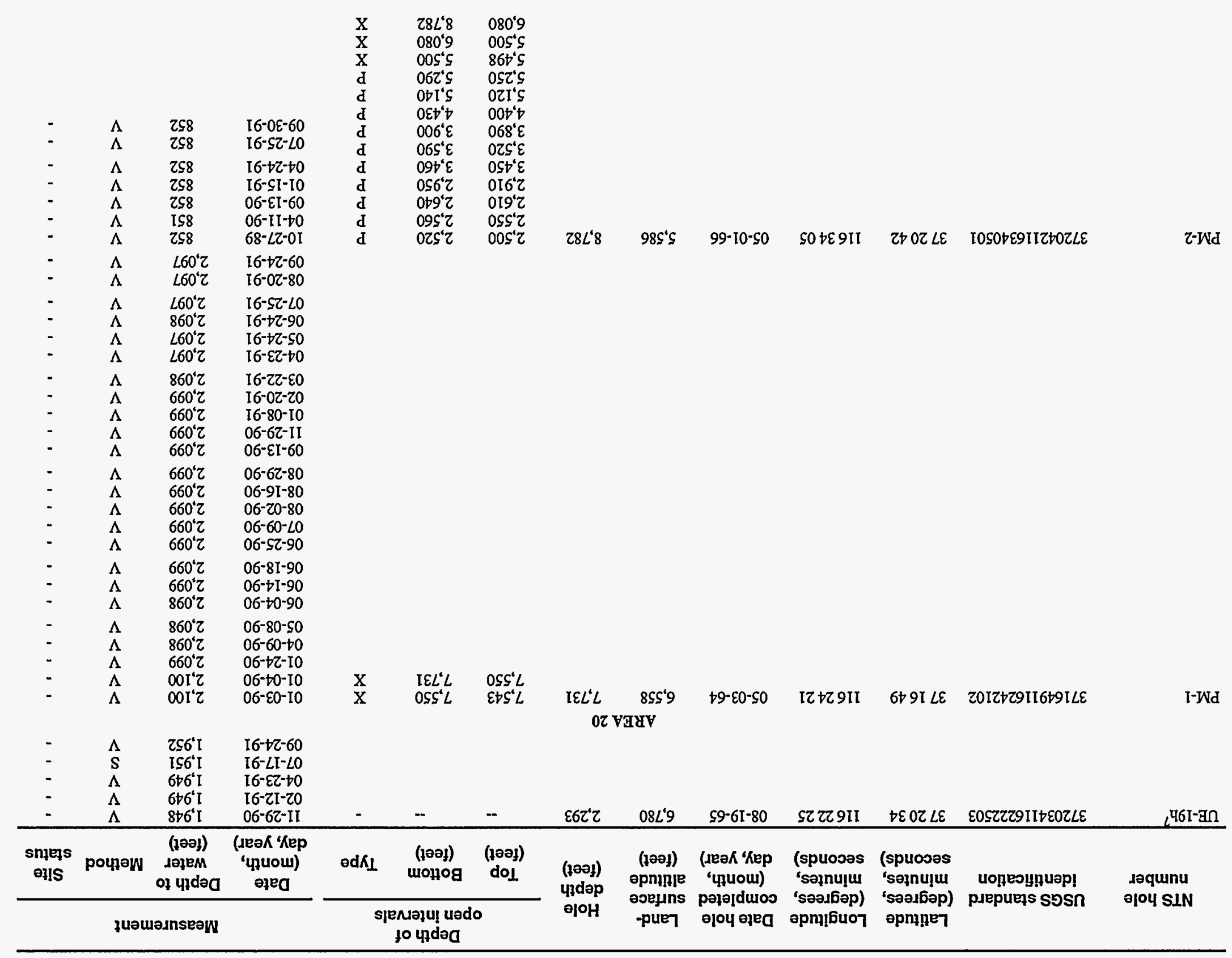


Table 1. Depth to water in wells and test holes at the Nevada Test Site (NTS) and the Faultless Site, Nye County, Nevada, water years 1990-91..-Continued

\begin{tabular}{|c|c|c|c|c|c|c|c|c|c|c|c|c|c|}
\hline \multirow{2}{*}{$\begin{array}{l}\text { NTS hole } \\
\text { number }\end{array}$} & \multirow{2}{*}{$\begin{array}{l}\text { USGS standard } \\
\text { identiffcation }\end{array}$} & \multirow{2}{*}{$\begin{array}{l}\text { Latitude } \\
\text { (degrees, } \\
\text { minutes, } \\
\text { seconds) }\end{array}$} & \multirow{2}{*}{$\begin{array}{l}\text { Longitude } \\
\text { (degrees, } \\
\text { minutes, } \\
\text { seconds) }\end{array}$} & \multirow{2}{*}{$\begin{array}{l}\text { Date hole } \\
\text { completed } \\
\text { (month, } \\
\text { day, year) }\end{array}$} & \multirow{2}{*}{$\begin{array}{l}\text { Land- } \\
\text { surface } \\
\text { altitude } \\
\text { (feet) }\end{array}$} & \multirow{2}{*}{$\begin{array}{l}\text { Hole } \\
\text { depth } \\
\text { (feet) }\end{array}$} & \multicolumn{3}{|c|}{$\begin{array}{c}\text { Depth of } \\
\text { open intervals }\end{array}$} & \multicolumn{4}{|c|}{ Measurement } \\
\hline & & & & & & & $\begin{array}{c}\text { Top } \\
\text { (feet) }\end{array}$ & $\begin{array}{l}\text { Bottom } \\
\text { (feet) }\end{array}$ & Type & $\begin{array}{c}\text { Date } \\
\text { (month, } \\
\text { day, year) }\end{array}$ & $\begin{array}{l}\text { Depth to } \\
\text { water } \\
\text { (feet) }\end{array}$ & Method & $\begin{array}{l}\text { Site } \\
\text { status }\end{array}$ \\
\hline U-20 WW & 371505116254501 & 371505 & 1162545 & $07-22-85$ & 6,468 & 3,268 & $\begin{array}{l}2,271 \\
3,199\end{array}$ & $\begin{array}{l}3,035 \\
3,268\end{array}$ & $\begin{array}{l}\mathrm{p} \\
\mathrm{X}\end{array}$ & $\begin{array}{l}07-22-91 \\
08-20-91 \\
09-09-91 \\
09-23-91\end{array}$ & $\begin{array}{l}2,058 \\
2,057 \\
2,057 \\
2,057\end{array}$ & $\begin{array}{l}\mathrm{V} \\
\mathrm{V} \\
\mathrm{V} \\
\mathrm{V}\end{array}$ & $\begin{array}{l}- \\
- \\
-\end{array}$ \\
\hline \multirow[t]{4}{*}{ U-20ax } & 371350116264701 & 371350 & 1162647 & $08-21-87$ & 6,536 & 2,200 & 62 & 2,200 & $x$ & $\begin{array}{l}10-11-89 \\
11-29-89 \\
04-09-90 \\
05-08-90 \\
06-01-90\end{array}$ & $\begin{array}{l}2,173 \\
2,174 \\
2,173 \\
2,173 \\
2,173\end{array}$ & $\begin{array}{l}\mathrm{V} \\
\mathrm{V} \\
\mathrm{V} \\
\mathrm{V} \\
\mathrm{V}\end{array}$ & $\begin{array}{l}- \\
- \\
-\end{array}$ \\
\hline & & & & & & & & & & $\begin{array}{l}07-03-90 \\
07-31-90 \\
08-15-90\end{array}$ & $\begin{array}{l}2,173 \\
2,174 \\
2,174\end{array}$ & $\begin{array}{l}\mathrm{V} \\
\mathrm{V} \\
\mathrm{V}\end{array}$ & - \\
\hline & & & & & & & & & & $\begin{array}{l}09-25-90 \\
11-23-90 \\
01-30-91 \\
02-20-91 \\
03-22-91\end{array}$ & $\begin{array}{l}2,174 \\
2,174 \\
2,174 \\
2,174 \\
2,174\end{array}$ & $\begin{array}{l}\text { V } \\
V \\
V \\
V \\
V\end{array}$ & $\begin{array}{l}- \\
- \\
- \\
-\end{array}$ \\
\hline & & & & & & & & & & $\begin{array}{l}04-23-91 \\
05-24-91 \\
06-26-91 \\
07-24-91 \\
08-20-91 \\
09-10-91\end{array}$ & $\begin{array}{l}2,171 \\
2,172 \\
2,172 \\
2,171 \\
2,172 \\
2,173\end{array}$ & $\begin{array}{l}V \\
V \\
V \\
V \\
V \\
V\end{array}$ & $\begin{array}{l}- \\
- \\
- \\
- \\
-\end{array}$ \\
\hline \multirow[t]{2}{*}{$\mathrm{U}-20 \mathrm{bb}{ }^{4}$} & 371452116293901 & 371452 & 1162939 & $03-23-88$ & 6,226 & 1,900 & $\begin{array}{l}36 \\
37\end{array}$ & $\begin{array}{r}37 \\
1,900\end{array}$ & $\begin{array}{l}\mathrm{X} \\
\mathrm{X}\end{array}$ & $\begin{array}{l}10-02-89 \\
10-10-89 \\
10-17-89 \\
10-23-89 \\
10-30-89\end{array}$ & $\begin{array}{l}1,748 \\
1,747 \\
1,746 \\
1,746 \\
1,745\end{array}$ & $\begin{array}{l}V \\
V \\
V \\
V \\
V\end{array}$ & $\begin{array}{l}- \\
- \\
- \\
-\end{array}$ \\
\hline & & & & & & & & & & $\begin{array}{l}11-01-89 \\
11-07-89 \\
11-08-89 \\
12-01-89 \\
12-18-89\end{array}$ & $\begin{array}{l}1,743 \\
1,743 \\
1,743 \\
1,741 \\
1,739\end{array}$ & $\begin{array}{l}V \\
V \\
V \\
V \\
V\end{array}$ & $\begin{array}{l}- \\
- \\
- \\
-\end{array}$ \\
\hline \multirow[t]{2}{*}{$U-20 b b^{4}$} & 371452116293902 & 371452 & 1162939 & $01-30-90$ & 6,226 & 2,220 & $\begin{array}{l}36 \\
37\end{array}$ & $\begin{array}{r}37 \\
2,220 \\
\end{array}$ & $\underset{\mathrm{X}}{\mathrm{X}}$ & $\begin{array}{l}02-13-90 \\
02-14-90 \\
02-16-90 \\
02-21-90 \\
03-01-90\end{array}$ & $\begin{array}{l}1,967 \\
1,973 \\
1,982 \\
1,998 \\
2,009\end{array}$ & $\begin{array}{l}V \\
V \\
V \\
V \\
V\end{array}$ & $\begin{array}{l}Z \\
- \\
- \\
- \\
-\end{array}$ \\
\hline & . & & & & & & & & & $\begin{array}{l}03-09-90 \\
03-22-90 \\
04-19-90\end{array}$ & $\begin{array}{l}2,014 \\
2,034 \\
2,050\end{array}$ & $\begin{array}{l}\mathrm{V} \\
\mathrm{V} \\
\mathrm{R}\end{array}$ & - \\
\hline
\end{tabular}


Table 1. Depth to water in wells and test holes at the Nevada Test Site (NTS) and the Faultess Site, Nye County, Nevada, water years 1990-91-Continued

\begin{tabular}{|c|c|c|c|c|c|c|c|c|c|c|c|c|c|}
\hline \multirow[b]{2}{*}{$\begin{array}{l}\text { NTS hole } \\
\text { number }\end{array}$} & \multirow[b]{2}{*}{$\begin{array}{c}\text { USGS standard } \\
\text { Identification } \\
.\end{array}$} & \multirow{2}{*}{$\begin{array}{c}\text { Latitude } \\
\text { (degrees, } \\
\text { minutes, } \\
\text { seconds) }\end{array}$} & \multirow{2}{*}{$\begin{array}{l}\text { Longitude } \\
\text { (degrees, } \\
\text { minutes, } \\
\text { seconds) }\end{array}$} & \multirow{2}{*}{$\begin{array}{c}\text { Date hole } \\
\text { completed } \\
\text { (month, } \\
\text { day, year) }\end{array}$} & \multirow{2}{*}{$\begin{array}{l}\text { Land- } \\
\text { surface } \\
\text { altitude } \\
\text { (feet) }\end{array}$} & \multirow{2}{*}{$\begin{array}{l}\text { Hole } \\
\text { depth } \\
\text { (feet) }\end{array}$} & \multicolumn{3}{|c|}{$\begin{array}{c}\text { Depth of } \\
\text { open intervals }\end{array}$} & \multicolumn{4}{|c|}{ Measurement } \\
\hline & & & & & & & $\begin{array}{l}\text { Top } \\
\text { (feet) }\end{array}$ & $\begin{array}{c}\text { Bottom } \\
\text { (feet) }\end{array}$ & Type & $\begin{array}{c}\text { Date } \\
\text { (month, } \\
\text { day, year) }\end{array}$ & $\begin{array}{l}\text { Depth to } \\
\text { water } \\
\text { (feet) }\end{array}$ & Method & $\begin{array}{l}\text { Site } \\
\text { status }\end{array}$ \\
\hline \multirow[t]{4}{*}{ U-20bb 1} & 371452116293903 & 371451 & 1162939 & $05-07-90$ & 6,227 & 2,322 & 62 & 2,345 & $\mathrm{x}$ & $\begin{array}{l}05-15-90 \\
05-16-90 \\
05-18-90 \\
05-25-90 \\
05-29-90\end{array}$ & $\begin{array}{l}2,028 \\
2,028 \\
2,027 \\
2,027 \\
2,027\end{array}$ & $\begin{array}{l}V \\
v \\
v \\
v \\
v\end{array}$ & $\begin{array}{l}- \\
\vdots \\
-\end{array}$ \\
\hline & & & & & & & & & & $\begin{array}{l}05-31-90 \\
06-04-90 \\
06-05-90 \\
06-07-90 \\
06-11-90\end{array}$ & $\begin{array}{l}2,027 \\
2,027 \\
2,027 \\
2,027 \\
2,027\end{array}$ & $\begin{array}{l}V \\
V \\
V \\
V \\
V\end{array}$ & $:$ \\
\hline & & & & & & & & & & $\begin{array}{l}06-14-90 \\
06-18-90 \\
06-25-90 \\
06-29-90 \\
07-02-90\end{array}$ & $\begin{array}{l}2,028 \\
2,028 \\
2,028 \\
2,028 \\
2,028\end{array}$ & $\begin{array}{l}V \\
V \\
V \\
V \\
V\end{array}$ & $\begin{array}{l}- \\
- \\
-\end{array}$ \\
\hline & & & & & & & & & & $\begin{array}{l}07-06-90 \\
07-09-90\end{array}$ & $\begin{array}{l}2,028 \\
2,028\end{array}$ & $\begin{array}{l}\mathrm{v} \\
\mathrm{v}\end{array}$ & $:$ \\
\hline U-20bd 1 & 371542116251301 & 371542 & 1162513 & $11-16-89$ & 6,486 & 2,402 & $\begin{array}{l}114 \\
120\end{array}$ & $\begin{array}{r}120 \\
2,402\end{array}$ & $\begin{array}{l}\mathrm{x} \\
\mathrm{x}\end{array}$ & $\begin{array}{l}01-09-90 \\
01-29-90 \\
01-31-90 \\
03-09-90 \\
03-14-90\end{array}$ & $\begin{array}{l}2,039 \\
2,039 \\
2,038 \\
2,038 \\
2,038\end{array}$ & $\begin{array}{l}\text { V } \\
\text { V } \\
\text { V } \\
\text { v } \\
\text { V }\end{array}$ & $\begin{array}{l}- \\
\vdots \\
-\end{array}$ \\
\hline U-20bd 2 & 371542116251202 & 371542 & 1162512 & $12-21-89$ & 6,551 & 2,450 & $\begin{array}{l}60 \\
63\end{array}$ & $\begin{array}{r}63 \\
2,450\end{array}$ & $\begin{array}{l}\mathrm{X} \\
\mathrm{x}\end{array}$ & $\begin{array}{l}01-09-90 \\
01-22-90 \\
01-31-90 \\
03-09-90 \\
03-14-90\end{array}$ & $\begin{array}{l}2,040 \\
2,039 \\
2,039 \\
2,037 \\
2,038\end{array}$ & $\begin{array}{l}V \\
V \\
V \\
V \\
V\end{array}$ & $\begin{array}{l}\mathrm{Z} \\
- \\
- \\
-\end{array}$ \\
\hline \multirow[t]{3}{*}{$\mathrm{U}-20 \mathrm{be}$} & 371332116254101 & 371332 & 1162541 & $06-12-89$ & 6,492 & 2,220 & $\begin{array}{l}51 \\
53\end{array}$ & $\begin{array}{r}53 \\
2,220\end{array}$ & $\begin{array}{l}\mathrm{x} \\
\mathrm{x}\end{array}$ & $\begin{array}{l}10-02-89 \\
10-10-89 \\
10-17-89 \\
10-23-89 \\
10-30-89\end{array}$ & $\begin{array}{l}2,206 \\
2,207 \\
2,207 \\
2,208 \\
2,209\end{array}$ & $\begin{array}{l}V \\
V \\
V \\
V \\
V\end{array}$ & $\begin{array}{l}- \\
- \\
-\end{array}$ \\
\hline & & & & & & & & & & $\begin{array}{l}11-09-89 \\
11-13-89 \\
11-27-89 \\
11-29-89 \\
12-04-89\end{array}$ & $\begin{array}{l}2,210 \\
2,210 \\
2,212 \\
2,211 \\
2,211\end{array}$ & $\begin{array}{l}\mathrm{V} \\
\mathrm{V} \\
\mathrm{V} \\
\mathrm{V} \\
\mathrm{V}\end{array}$ & $\begin{array}{l}- \\
- \\
-\end{array}$ \\
\hline & & & & & & & & & & $\begin{array}{l}12-11-89 \\
12-18-89 \\
12-29-89 \\
01-09-90 \\
01-22-90\end{array}$ & $\begin{array}{l}2,211 \\
2,211 \\
2,212 \\
2,212 \\
2,214\end{array}$ & $\begin{array}{l}V \\
V \\
V \\
V \\
V\end{array}$ & $\begin{array}{l}- \\
- \\
-\end{array}$ \\
\hline
\end{tabular}


Table 1. Depth to water in wells and test holes at the Nevada Test Site (NTS) and the Faultless Site, Nye County, Nevada, water years 1990-91--Continued

\begin{tabular}{|c|c|c|c|c|c|c|c|c|c|c|c|c|c|}
\hline \multirow{2}{*}{$\begin{array}{l}\text { NTS hole } \\
\text { number }\end{array}$} & \multirow{2}{*}{$\begin{array}{l}\text { USGS standard } \\
\text { Ident/fication }\end{array}$} & \multirow{2}{*}{$\begin{array}{l}\text { Latitude } \\
\text { (degrees, } \\
\text { minutes, } \\
\text { seconds) }\end{array}$} & \multirow{2}{*}{$\begin{array}{l}\text { Longitude } \\
\text { (degrees, } \\
\text { minutes, } \\
\text { seconds) }\end{array}$} & \multirow{2}{*}{$\begin{array}{l}\text { Date hole } \\
\text { completed } \\
\text { (month, } \\
\text { day, year) }\end{array}$} & \multirow{2}{*}{$\begin{array}{l}\text { Land- } \\
\text { surface } \\
\text { altitude } \\
\text { (feet) }\end{array}$} & \multirow{2}{*}{$\begin{array}{l}\text { Hole } \\
\text { depth } \\
\text { (feet) }\end{array}$} & \multicolumn{3}{|c|}{$\begin{array}{c}\text { Depth of } \\
\text { open intervals }\end{array}$} & \multicolumn{4}{|c|}{ Measurement } \\
\hline & & & & & & & $\begin{array}{l}\text { Top } \\
\text { (feet) }\end{array}$ & $\begin{array}{c}\text { Bottom } \\
\text { (feet) }\end{array}$ & Type & $\begin{array}{c}\text { Date } \\
\text { (month, } \\
\text { day, year) }\end{array}$ & $\begin{array}{l}\text { Depth to } \\
\text { water } \\
\text { (feet) }\end{array}$ & Method & $\begin{array}{c}\text { Site } \\
\text { status }\end{array}$ \\
\hline \multirow[t]{3}{*}{ U-20be } & 371332116254101 & 371332 & 1162541 & $06-12-89$ & 6,492 & 2,220 & $\begin{array}{l}51 \\
53\end{array}$ & $\begin{array}{r}53 \\
2,220\end{array}$ & $\begin{array}{l}\bar{X} \\
\mathrm{X}\end{array}$ & $\begin{array}{l}01-29-90 \\
02-12-90 \\
04-09-90 \\
05-07-90 \\
06-01-90\end{array}$ & $\begin{array}{l}2,213 \\
2,213 \\
2,213 \\
2,213 \\
2,214\end{array}$ & $\begin{array}{l}\mathrm{V} \\
\mathrm{V} \\
\mathrm{V} \\
\mathrm{V} \\
\mathrm{V}\end{array}$ & $\begin{array}{l}- \\
- \\
-\end{array}$ \\
\hline & & & & & & & & & & $\begin{array}{l}07-03-90 \\
07-31-90 \\
08-15-90 \\
08-29-90 \\
09-25-90\end{array}$ & $\begin{array}{l}2,214 \\
2,214 \\
2,214 \\
2,214 \\
2,215\end{array}$ & $\begin{array}{l}V \\
V \\
V \\
V \\
V\end{array}$ & $\begin{array}{l}- \\
\dot{z} \\
\dot{z}\end{array}$ \\
\hline & & & & & & & & & & $\begin{array}{l}11-23-90 \\
01-08-91 \\
04-30-91 \\
06-05-91\end{array}$ & $\begin{array}{l}2,215 \\
2,215 \\
2,215 \\
2,215\end{array}$ & $\begin{array}{l}\mathrm{V} \\
\mathrm{V} \\
\mathrm{V} \\
\mathrm{V}\end{array}$ & $\begin{array}{l}Z \\
- \\
-\end{array}$ \\
\hline \multirow[t]{5}{*}{$\mathrm{U}-20 \mathrm{bf}{ }^{8}$} & 371444116263001 & 371444 & 1162630 & $08-23-89$ & 6,522 & 2,140 & $\begin{array}{l}48 \\
49\end{array}$ & $\begin{array}{r}49 \\
2,250\end{array}$ & $\begin{array}{l}\mathrm{X} \\
\mathrm{X}\end{array}$ & $\begin{array}{l}10-10-89 \\
10-17-89 \\
10-24-89 \\
10-30-89 \\
11-09-89\end{array}$ & $\begin{array}{l}2,131 \\
2,131 \\
2,131 \\
2,131 \\
2,131\end{array}$ & $\begin{array}{l}\mathrm{V} \\
\mathrm{V} \\
\mathrm{V} \\
\mathrm{V} \\
\mathrm{V}\end{array}$ & $:$ \\
\hline & & & & & & & & & & $\begin{array}{l}11-13-89 \\
11-27-89 \\
11-29-89 \\
12-04-89 \\
12-11-89\end{array}$ & $\begin{array}{l}2,131 \\
2,132 \\
2,132 \\
2,132 \\
2,132\end{array}$ & $\begin{array}{l}\mathrm{V} \\
\mathrm{V} \\
\mathrm{V} \\
\mathrm{V} \\
\mathrm{V}\end{array}$ & $:$ \\
\hline & & & & & & & & & & $\begin{array}{l}12-18-89 \\
12-29-89 \\
01-08-90 \\
01-24-90 \\
01-29-90\end{array}$ & $\begin{array}{l}2,131 \\
2,132 \\
2,132 \\
2,132 \\
2,131\end{array}$ & $\begin{array}{l}V \\
V \\
V \\
V \\
V\end{array}$ & $\begin{array}{l}- \\
- \\
-\end{array}$ \\
\hline & & & & & & & & & & $\begin{array}{l}02-13-90 \\
04-04-90 \\
05-07-90 \\
06-01-90 \\
06-14-90\end{array}$ & $\begin{array}{l}2,130 \\
2,132 \\
2,132 \\
2,132 \\
2,133\end{array}$ & $\begin{array}{l}V \\
V \\
V \\
V \\
V\end{array}$ & $\begin{array}{l}- \\
\vdots \\
-\end{array}$ \\
\hline & & & & & & & & & & $\begin{array}{l}06-18-90 \\
06-25-90 \\
07-02-90 \\
07-12-90 \\
07-31-90\end{array}$ & $\begin{array}{l}2,133 \\
2,134 \\
2,134 \\
2,134 \\
2,134\end{array}$ & $\begin{array}{l}\mathrm{V} \\
\mathrm{V} \\
\mathrm{V} \\
\mathrm{V} \\
\mathrm{V}\end{array}$ & $\begin{array}{l}- \\
- \\
-\end{array}$ \\
\hline
\end{tabular}




\begin{tabular}{|c|c|c|c|c|c|c|c|c|c|c|c|c|c|}
\hline & & & & & & & & & & & & & \\
\hline$\overline{-}$ & $\Lambda$ & $\nabla \varepsilon L^{L} T$ & $I 6-\angle Z-60$ & & & & & & & & & & \\
\hline$\because$ & $\Lambda$ & $\begin{array}{l}S \varepsilon L^{\prime} I \\
t \varepsilon L^{\prime} I\end{array}$ & $\begin{array}{l}16-9 z-50 \\
06-z 0-50\end{array}$ & $\mathrm{x}$ & $00 t^{\natural} \varepsilon$ & $6 L \varepsilon^{\natural} \varepsilon$ & & & & & & & \\
\hline & & $S E L^{\circ} i$ & $68-92-0 \mathrm{I}$ & $\mathrm{x}$ & $6 L \varepsilon^{\prime} \varepsilon$ & 0 OSI $\varepsilon$ & $00 t^{\circ} \varepsilon$ & $\varepsilon t I^{\top} t$ & Z९-ZI-90 & 6S $909 I \mathrm{II}$ & $\downarrow \varepsilon S \triangleright 9 \varepsilon$ & 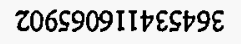 & $d-M I$ \\
\hline & & & & & & & & gyv & & & & & \\
\hline : & $\Lambda$ & $\begin{array}{l}0+0^{\circ} z \\
0+0^{\circ} z\end{array}$ & $\begin{array}{l}\tau 6-\varepsilon z-60 \\
\tau \sigma-6 z-\angle 0\end{array}$ & & & & & & & & & & \\
\hline - & $\Lambda$ & $8 \varepsilon 0^{\circ} z$ & I $6-0 \varepsilon-t 0$ & & & & & & & & & & \\
\hline$:$ & $\hat{\Lambda}$ & $\begin{array}{l}6 \varepsilon 0^{\circ} Z \\
L E 0^{\circ} Z\end{array}$ & $\begin{array}{l}I 6-80-Z 0 \\
06-\varepsilon Z-\text { II }\end{array}$ & & & & & & & & & & \\
\hline - & $\Lambda$ & $\$ \varepsilon 0^{\circ} z$ & $06-60-\angle 0$ & & & & & & & & & & \\
\hline - & $\ddot{\Lambda}$ & $9 \varepsilon 0^{\circ} z$ & $06-50-90$ & & & & & & & & & & \\
\hline : & $\Lambda$ & 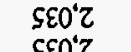 & $06-80-50$ & $\underset{\mathrm{X}}{\mathrm{X}}$ & $\downarrow \varepsilon 8^{\circ} z$ & $\begin{array}{l}\angle 0 b^{*} Z \\
c 7 c^{\prime} Z\end{array}$ & & & & & & & \\
\hline- & $\hat{\Lambda}$ & $\begin{array}{l}\varsigma \varepsilon 0^{\circ} Z \\
\varsigma \varepsilon 0^{\circ} z\end{array}$ & $\begin{array}{l}06-Z I-t 0 \\
68-\mathrm{II}-0 \mathrm{I}\end{array}$ & $\begin{array}{l}x \\
x\end{array}$ & $\begin{array}{l}\text { Lot'Z } \\
\varepsilon z \varepsilon^{\prime} Z\end{array}$ & $\begin{array}{l}\varepsilon z \varepsilon z \\
z 8 z^{2} z\end{array}$ & $\downarrow \varepsilon 8^{\prime} Z$ & $19 t^{\prime} 9$ & $\angle 8=0 I-90$ & 6I SZ9II & $\varsigma Z \triangleright I L \varepsilon$ & Z06ISZ9IISZDI LE & I น นоz-घก \\
\hline : & $\hat{\Lambda}$ & $\begin{array}{l}850^{\circ} z \\
850^{\circ} z\end{array}$ & $\begin{array}{l}{[6-\varepsilon z-60} \\
{[6-6 z-\angle 0}\end{array}$ & & & & & & & & & & \\
\hline - & $\Lambda$ & $8+0^{\circ} \mathrm{Z}$ & I6-0ع-†0 & & & & & & & & & & \\
\hline - & $\Lambda$ & $800^{\circ} \mathrm{z}$ & โ6-ZI-Z0 & & & & & & & & & & \\
\hline - & $\Lambda$ & $\begin{array}{l}9+0^{\circ} z \\
\varepsilon \neq 0^{\circ} z\end{array}$ & $06-\varepsilon z-\tau I$ & & & & & & & & & & \\
\hline- & $\Lambda$ & $\begin{array}{l}\varepsilon 072 \\
\$ \$ 0^{\circ} z\end{array}$ & $\begin{array}{l}06-60-\angle 0 \\
06-50-90\end{array}$ & & & & & & & & & & \\
\hline 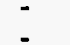 & $\Lambda$ & t & $06-80-50$ & & & & & & & & & & \\
\hline - & $\hat{\Lambda}$ & $\begin{array}{l}\qquad \neq 0 ? \\
t+0^{\circ} z\end{array}$ & $\begin{array}{l}06-Z I-t 0 \\
68-1 T-0 I\end{array}$ & d & $\$ 66^{6} z$ & $\$ 99^{\circ} \mathrm{z}$ & $\$ 20^{\circ} \varepsilon$ & $89 t^{*} 9$ & 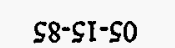 & †Z SZ9II & $s \tau \pitchfork I L \varepsilon$ & IOわZSZ9IISZヤILE & 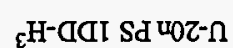 \\
\hline$=$ & $\Lambda$ & $z \varepsilon I^{2} r$ & {$[6-7 z-60$} & & & & & & & & & & \\
\hline - & $\hat{\Lambda}$ & $\begin{array}{l}\text { IEI? } \\
\text { IEI' } 2\end{array}$ & $\begin{array}{l}6-01-60 \\
16-t-80\end{array}$ & & & & & & & & & & \\
\hline - & $\Lambda$ & Oहा' & โ6-9z-90 & & & & & & & & & & \\
\hline & $\Lambda$ & $0 \varepsilon I^{2} z$ & $16-t z-50$ & & & & & & & & & & \\
\hline - & $\Lambda$ & $0 \varepsilon \varepsilon^{2} z$ & I6-๕Z-๖0 & & & & & & & & & & \\
\hline - & $\Lambda$ & $\begin{array}{l}8 \mathrm{ZI} Z \\
6 Z \mathrm{I}^{\circ} \mathrm{Z}\end{array}$ & $\begin{array}{l}I 6-z z-\varepsilon 0 \\
\tau 6-0 z-z 0\end{array}$ & & & & & & & & & & \\
\hline - & $\Lambda$ & $62 I^{\prime} Z$ & I $6-0 \varepsilon-10$ & & & & & & & & & & \\
\hline$\overline{-}$ & $\Lambda$ & OEI'Z & $\mathrm{I} 6-\mathrm{bI}-\mathrm{I0}$ & $\mathrm{x}$ & $00 z^{\prime} z$ & ots & & & & & & & \\
\hline $\mathbf{Z}$ & $\Lambda$ & OEI'z & โ6-80-โ0 & $\mathrm{x}$ & $0+5$ & $8 s$ & $00 z^{\prime} z$ & L9S'9 & $06-6 I-2 I$ & $6 Z+Z 911$ & $\not \mathfrak{l} \neq \mathfrak{l} L \varepsilon$ & I06ZฤZ9IIฤIヤILE & sq0z-n \\
\hline$\sigma$ & $\Lambda$ & $980^{\circ} Z$ & $16-0 \varepsilon-10$ & & & & & & & & & & \\
\hline $\mathrm{d}$ & $\Lambda$ & $980^{\circ} \mathrm{Z}$ & $16-t z-10$ & & & & & & & & & & \\
\hline $\mathrm{Z}$ & $\Lambda$ & $980^{\circ} \mathrm{Z}$ & I6-80-I0 & & & & & & & & & & \\
\hline$\underline{-}$ & $\hat{\Lambda}$ & 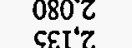 & 06-EZ-II & & & & & & & & & & \\
\hline - & $\Lambda$ & SEI'Z & $\begin{array}{l}06-6 Z-80 \\
06-\varsigma \uparrow-80\end{array}$ & $\stackrel{x}{x}$ & $6 t$ & $8 t$ & $0 x^{2} z$ & ZZS'9 & $68-\varepsilon z-80$ & 0E 9Z9II & $t \mapsto I L \varepsilon$ & 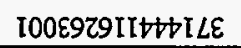 & $890 z-n$ \\
\hline & & (120, & (sеәК 'кер & & & & & & & & & & \\
\hline อ॥!s & рочтаW & $\begin{array}{l}\text { dojem } \\
\text { of पldad }\end{array}$ & $\begin{array}{l}\text { 'दjuoul) } \\
\text { өqea }\end{array}$ & edK $\perp$ & woHog & dol & $\begin{array}{l}\text { (1003) } \\
\text { uldap }\end{array}$ & epn?n] & (ч) оош) & 'sep̣nụ̂u & 'sąnuḷu & uoppeo!nguap! & sequnu \\
\hline & & dajnsea & & & $\begin{array}{l}\text { napul uac } \\
\text { go yidad }\end{array}$ & & $\mathrm{OOH}$ & -puet & 빈 빔ㅁ & өpח!!6u०7 & өpn!ne7 & 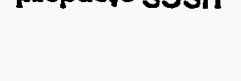 & \\
\hline
\end{tabular}


Table 1. Depth to water in wells and test holes at the Nevada Test Site (NTS) and the Faultless Site, Nye County, Nevada, water years 1990-91--Continued

\begin{tabular}{|c|c|c|c|c|c|c|c|c|c|c|c|c|c|}
\hline \multirow{2}{*}{$\begin{array}{l}\text { NTS hole } \\
\text { number }\end{array}$} & \multirow{2}{*}{$\begin{array}{l}\text { USGS standard } \\
\text { Identification }\end{array}$} & \multirow{2}{*}{$\begin{array}{r}\text { Latitude } \\
\text { (degrees, } \\
\text { minutes, } \\
\text { seconds) }\end{array}$} & \multirow{2}{*}{$\begin{array}{l}\text { Longitude } \\
\text { (degrees, } \\
\text { minutes, } \\
\text { seconds) }\end{array}$} & \multirow{2}{*}{$\begin{array}{l}\text { Date hole } \\
\text { completed } \\
\text { (month, } \\
\text { day, year) }\end{array}$} & \multirow{2}{*}{$\begin{array}{l}\text { Land- } \\
\text { surface } \\
\text { altitude } \\
\text { (feet) }\end{array}$} & \multirow{2}{*}{$\begin{array}{l}\text { Hole } \\
\text { depth } \\
\text { (feet) }\end{array}$} & \multicolumn{3}{|c|}{$\begin{array}{c}\text { Depth of } \\
\text { open intervals }\end{array}$} & \multicolumn{4}{|c|}{ Measurement } \\
\hline & & & & & & & $\begin{array}{c}\text { Top } \\
\text { (feet) }\end{array}$ & $\begin{array}{c}\text { Bottom } \\
\text { (feet) }\end{array}$ & Type & $\begin{array}{c}\text { Date } \\
\text { (month, } \\
\text { day, year) }\end{array}$ & $\begin{array}{l}\text { Depth to } \\
\text { water } \\
\text { (feet) }\end{array}$ & Method & $\begin{array}{l}\text { Slte } \\
\text { status }\end{array}$ \\
\hline \multicolumn{14}{|c|}{ FRENCHMAN FLAT ADJACENT TO NEVADA TEST SITE } \\
\hline TW-3 & 364830115512601 & 364830 & 1155126 & 05-09-62 & 3,489 & 1,356 & 1,192 & 1,516 & $P$ & $\begin{array}{l}06-09-90 \\
11-16-90 \\
04-26-91 \\
09-27-91\end{array}$ & $\begin{array}{l}1,102 \\
1,103 \\
1,103 \\
1,103\end{array}$ & $\begin{array}{l}\mathrm{V} \\
\mathrm{V} \\
\mathrm{V} \\
\mathrm{v}\end{array}$ & 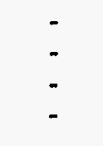 \\
\hline \multicolumn{14}{|c|}{ PAHUTE MESA ADJACENT TO NEVADA TEST SITE } \\
\hline PM-3 & 371421116333702 & 371421 & 1163337 & 09-19-88 & 5,823 & 3,019 & $\begin{array}{l}1,473 \\
1,647\end{array}$ & $\begin{array}{l}1,647 \\
3,019\end{array}$ & $\begin{array}{l}\mathrm{x} \\
\mathrm{x}\end{array}$ & $\begin{array}{l}10-24-89 \\
01-05-90 \\
04-11-90 \\
07-02-90 \\
11-23-90\end{array}$ & $\begin{array}{l}1,457 \\
1,459 \\
1,458 \\
1,458 \\
1,459\end{array}$ & $\begin{array}{l}\text { V } \\
\text { V } \\
\text { V } \\
\text { V } \\
\text { V }\end{array}$ & $\begin{array}{l}- \\
\vdots \\
-\end{array}$ \\
\hline & & & & & OT CREEI & VALLE & & & & $\begin{array}{l}01-15-91 \\
04-24-91 \\
07-25-91 \\
09-30-91\end{array}$ & $\begin{array}{l}1,458 \\
1,457 \\
1,458 \\
1,458 \\
\end{array}$ & $\begin{array}{l}\text { V } \\
\text { V } \\
\text { V } \\
\text { V }\end{array}$ & $\begin{array}{l}- \\
-\end{array}$ \\
\hline HTH-1 & 383734116124501 & 383735 & 1161245 & $07-23-67$ & 6,011 & 3,695 & $\begin{array}{r}150 \\
250 \\
355 \\
435 \\
700 \\
775 \\
950 \\
1,050 \\
1,400 \\
1,660 \\
1,850 \\
2,200 \\
2,400 \\
2,640 \\
2,950 \\
3,590\end{array}$ & $\begin{array}{r}250 \\
345 \\
435 \\
510 \\
775 \\
850 \\
1,050 \\
1,150 \\
1,500 \\
1,720 \\
1,980 \\
2,300 \\
2,460 \\
2,710 \\
3,010 \\
3,665\end{array}$ & $\begin{array}{l}\mathrm{P} \\
\mathrm{P} \\
\mathrm{P} \\
\mathrm{P} \\
\mathrm{P} \\
\mathrm{P} \\
\mathrm{P} \\
\mathrm{P} \\
\mathrm{P} \\
\mathrm{P} \\
\mathrm{P} \\
\mathrm{P} \\
\mathrm{P} \\
\mathrm{P} \\
\mathrm{P} \\
\mathrm{P}\end{array}$ & $\begin{array}{l}10-31-89 \\
04-05-90 \\
12-06-90 \\
04-25-91 \\
09-19-91\end{array}$ & $\begin{array}{l}532 \\
531 \\
533 \\
532 \\
532\end{array}$ & $\begin{array}{l}\mathrm{V} \\
\mathrm{V} \\
\mathrm{T} \\
\mathrm{T} \\
\mathrm{T}\end{array}$ & $\begin{array}{l}- \\
- \\
-\end{array}$ \\
\hline \multirow[t]{2}{*}{ HTH-2 } & 383734116124502 & 383740 & 1161247 & $08-12-67$ & 6,025 & 1,000 & 504 & 1,000 & $P$ & $\begin{array}{l}10-31-89 \\
04-05-90 \\
12-06-90 \\
03-06-91 \\
04-25-91\end{array}$ & $\begin{array}{l}552 \\
552 \\
554 \\
553 \\
552\end{array}$ & $\begin{array}{l}\mathrm{V} \\
\mathrm{V} \\
\mathrm{T} \\
\mathrm{T} \\
\mathrm{T}\end{array}$ & $\begin{array}{l}- \\
- \\
- \\
-\end{array}$ \\
\hline & & & & & & & & & & $\begin{array}{l}06-26-91 \\
09-19-91\end{array}$ & $\begin{array}{l}553 \\
553\end{array}$ & $\begin{array}{l}\mathrm{T} \\
\mathrm{T}\end{array}$ & - \\
\hline
\end{tabular}


Table 1. Depth to water in wells and test holes at the Nevada Test Site (NTS) and the Faultless Site, Nye County, Nevada, water years 1990-91--Continued

\begin{tabular}{|c|c|c|c|c|c|c|c|c|c|c|c|c|c|}
\hline \multirow{2}{*}{$\begin{array}{l}\text { NTS hole } \\
\text { number }\end{array}$} & \multirow{2}{*}{$\begin{array}{l}\text { USGS standard } \\
\text { identification }\end{array}$} & \multirow{2}{*}{$\begin{array}{l}\text { Latitude } \\
\text { (degrees, } \\
\text { minutes, } \\
\text { seconds) }\end{array}$} & \multirow{2}{*}{$\begin{array}{l}\text { Longitude } \\
\text { (degrees, } \\
\text { minutes, } \\
\text { seconds) }\end{array}$} & \multirow{2}{*}{$\begin{array}{l}\text { Date hole } \\
\text { completed } \\
\text { (month, } \\
\text { day, year) }\end{array}$} & \multirow{2}{*}{$\begin{array}{l}\text { Land- } \\
\text { surface } \\
\text { altitude } \\
\text { (feet) }\end{array}$} & \multirow{2}{*}{$\begin{array}{l}\text { Hole } \\
\text { depth } \\
\text { (feet) }\end{array}$} & \multicolumn{3}{|c|}{$\begin{array}{c}\text { Depth of } \\
\text { open intervals }\end{array}$} & \multicolumn{4}{|c|}{ Measurement } \\
\hline & & & & & & & $\begin{array}{c}\text { Top } \\
\text { (feet) }\end{array}$ & $\begin{array}{c}\text { Bottom } \\
\text { (feet) }\end{array}$ & Type & $\begin{array}{c}\text { Date } \\
\text { (month, } \\
\text { day, year) }\end{array}$ & $\begin{array}{c}\text { Depth to } \\
\text { water } \\
\text { (feet) }\end{array}$ & Method & $\begin{array}{l}\text { Site } \\
\text { status }\end{array}$ \\
\hline \multirow[t]{2}{*}{ UC-1-P-2SR } & 383806116125951 & 383806 & 1161254 & $04-06-68$ & 6,084 & 2,734 & $\begin{array}{l}1,148 \\
1,148\end{array}$ & $\begin{array}{l}1,945 \\
2,790\end{array}$ & $\begin{array}{l}\mathrm{P} \\
\mathrm{P}\end{array}$ & $\begin{array}{l}10-31-89 \\
04-05-90 \\
11-13-90 \\
12-06-90 \\
03-06-91\end{array}$ & $\begin{array}{l}831 \\
817 \\
799 \\
798 \\
780\end{array}$ & $\begin{array}{l}V \\
V \\
T \\
T \\
T\end{array}$ & $\begin{array}{l}- \\
- \\
- \\
-\end{array}$ \\
\hline & & & & & & & & & & $\begin{array}{l}04-25-91 \\
06-26-91 \\
09-19-91\end{array}$ & $\begin{array}{l}788 \\
786 \\
775\end{array}$ & $\begin{array}{l}T \\
T \\
T\end{array}$ & - \\
\hline
\end{tabular}

${ }^{1}$ Depth of open intervals includes dual-access tubings.

${ }^{2}$ Depth of open intervals indicates multiple casings within borehole.

${ }^{3}$ Depth-to-water measurements corrected for borehole deviation from vertical; construction information not corrected for borehole deviation from vertical.

${ }^{4}$ Repeated NTS hole numbers reflect more that one depth-to-water measurement interval.

5 Packers set from 1,610 to 1,619 feet and from 1,721 to 1,728 feet. Measurements reflect interval between packers from 1,619 to 1,721 feet.

${ }^{6}$ Depth of open intervals reflect casing being cut numerous times in attempt to remove casing segment from borehole.

${ }^{7}$ Measurements may reflect occluded water. Cement plug at 2,293 feet; 28 feet inside the casing. The casing is not perforated.

8 Hole backfilled to 2,140 feet with Buckboard Mesa aggregate on November 5, 1990. 
Table 2. Depth to water in ground-water supply wells at the Nevada Test Site (NTS), Nye County, Nevada, calendar years 1951-91

NTS hole number--Drill-hole number assigned by Raytheon Services Nevada; see section "Site Designations" in text.

USGS standard identification--U.S. Geological Survey site designation; see section "Site Designations" in text.

Date well completed--Date that borehole constnuction work ceased; see section "Measurements" in text.

Land-surface altitude--Datum is sea level. Value may not represent current altitude; see section "Depth to Water" in text.

Well depth--Datum is land surface. Represents most recent available accessible depth, see section "Measurements" in text.

Depth of open intervals--Datum is land surface. Bottom of deepest open interval may be deeper than present accessible hole depth. Type: $\mathrm{P}$, perforaled or slotted casing; $\mathrm{X}$, open (uncased) hole.

Measurement--Datum is land surface. Value may not represent static water level; see section "Depth to Water" in text. Method: A, airline; $L$, interpreted from geophysical logs; $R$, depth to water reported (measurement method not known); S, steel tape; T, electric tape; V, iron-horse or wire-line device. Site status: $P$, site was being pumped; $R$, site was recently pumped or swabbed; $S$, nearby site was being pumped or swabbed; $Z$, other conditions not included in standard site status codes, see section "Measurements" in text.

\begin{tabular}{|c|c|c|c|c|c|c|c|c|c|c|c|c|c|}
\hline \multirow{2}{*}{$\begin{array}{c}\text { NTS } \\
\text { hole } \\
\text { number }\end{array}$} & \multirow{2}{*}{$\begin{array}{l}\text { USGS standard } \\
\text { identification }\end{array}$} & \multirow{2}{*}{$\begin{array}{l}\text { Latitude } \\
\text { (degrees, } \\
\text { minutes, } \\
\text { seconds) }\end{array}$} & \multirow{2}{*}{$\begin{array}{l}\text { Longitude } \\
\text { (degrees, } \\
\text { minutes, } \\
\text { seconds) }\end{array}$} & \multirow{2}{*}{$\begin{array}{l}\text { Date well } \\
\text { completed } \\
\text { (month, } \\
\text { day, year) }\end{array}$} & \multirow{2}{*}{$\begin{array}{l}\text { Land- } \\
\text { surface } \\
\text { altitude } \\
\text { (feet) }\end{array}$} & \multirow{2}{*}{$\begin{array}{l}\text { Well } \\
\text { depth } \\
\text { (feet) }\end{array}$} & \multicolumn{3}{|c|}{$\begin{array}{c}\text { Depth of } \\
\text { open interval }\end{array}$} & \multicolumn{4}{|c|}{ Measurement } \\
\hline & & & & & & & $\begin{array}{l}\text { Top } \\
\text { (feet) }\end{array}$ & $\begin{array}{c}\text { Bottom } \\
\text { (feet) }\end{array}$ & Type & $\begin{array}{c}\text { Date } \\
\text { (month, } \\
\text { day, year) }\end{array}$ & $\begin{array}{l}\text { Depth to } \\
\text { water } \\
\text { (feet) }\end{array}$ & Method & $\begin{array}{l}\text { Site } \\
\text { status }\end{array}$ \\
\hline \multicolumn{14}{|c|}{ AREA 1} \\
\hline UE-1r WW & 370142116033301 & 370142 & 1160333 & $01-16-84$ & 4,042 & 3,145 & $\begin{array}{l}2,319 \\
2,350\end{array}$ & $\begin{array}{l}2,350 \\
4,182\end{array}$ & $\begin{array}{l}\mathrm{X} \\
\mathrm{X}\end{array}$ & $\begin{array}{l}01-17-84 \\
01-18-84 \\
01-28-84\end{array}$ & $\begin{array}{l}1,769 \\
1,769 \\
1,626\end{array}$ & $\begin{array}{l}\text { L } \\
\text { L } \\
\text { L }\end{array}$ & - \\
\hline \multicolumn{14}{|c|}{ AREA 2} \\
\hline$W W-2^{1,2}$ & 370958116051501 & 370958 & 1160515 & $03-24-61$ & 4,470 & 2,045 & 1,465 & 2,045 & $X$ & $03-28-61$ & 1,915 & $\mathbf{R}$ & - \\
\hline$W W-2^{1,2}$ & 370958116051502 & 370958 & 1160515 & $05-30-61$ & 4,470 & 2,373 & 2,121 & 2,373 & $\mathrm{X}$ & -- & -. & - & - \\
\hline$W W-2^{1,2}$ & 370958116051503 & 370958 & 1160515 & $06-08-61$ & 4,470 & 2,535 & 2,121 & 2,535 & $\mathrm{x}$ & $\begin{array}{l}06-20-61 \\
08-18-61 \\
10-04-61 \\
10-23-61\end{array}$ & $\begin{array}{l}1,958 \\
1,974 \\
1,979 \\
1,979\end{array}$ & $\begin{array}{l}\mathrm{V} \\
\mathrm{V} \\
\mathrm{V} \\
\mathrm{R}\end{array}$ & $\begin{array}{l}- \\
- \\
-\end{array}$ \\
\hline$W W-2^{1,2}$ & 370958116051504 & 370958 & 1160515 & $12-18-61$ & 4,470 & 2,571 & 2,550 & 2,571 & $\mathrm{X}$ & -- & -. & - & - \\
\hline$W W-2^{1,2}$ & 370958116051505 & 370958 & 1160515 & $12-20-61$ & 4,470 & 2,593 & 2,550 & 2,593 & $\mathrm{x}$ & -- & -- & - & - \\
\hline$W W-2^{1,2}$ & 370958116051506 & 370958 & 1160515 & $12-26-61$ & 4,470 & 2,634 & 2,550 & 2,634 & $\mathrm{x}$ & - & -- & - & - \\
\hline$W W-2^{1,2}$ & 370958116051507 & 370958 & 1160515 & $12-30-61$ & 4,470 & 2,703 & 2,550 & 2,703 & $\mathrm{x}$ & -- & - & - & - \\
\hline$W W-2^{1,2}$ & 370958116051508 & 370958 & 1160515 & $01-12-62$ & 4,470 & 2,896 & 2,550 & 2,896 & $\mathrm{X}$ & $01-15-62$ & 2,056 & $\mathrm{R}$ & - \\
\hline$W W-2^{1,2}$ & 370958116051509 & 370958 & 1160515 & $01-19-62$ & 4,470 & 3,095 & 2,550 & 3,095 & $\mathrm{X}$ & -. & -- & - & - \\
\hline$W W-2^{1,2}$ & 370958116051510 & 370958 & 1160515 & $01-28-62$ & 4,470 & 3,295 & 2,550 & 3,295 & $\mathrm{x}$ & -- & - & - & - \\
\hline$W W-2^{1,2}$ & 370958116051511 & 370958 & 1160515 & $02-09-62$ & 4,470 & 3,422 & $\dot{2}, 550$ & 3,422 & $\mathrm{X}$ & $02-15-62$ & 2,053 & $\mathrm{~V}$ & $\mathrm{R}$ \\
\hline$W W-2^{1,2}$ & 370958116051512 & 370958 & 1160515 & $03-11-62$ & 4,470 & 3,422 & $\begin{array}{l}2,700 \\
3,164\end{array}$ & $\begin{array}{l}2,950 \\
3,412\end{array}$ & $\begin{array}{l}\mathrm{P} \\
\mathrm{P}\end{array}$ & $\begin{array}{l}03-16-62 \\
03-20-62 \\
03-23-62 \\
11-21-63 \\
12-04-64 \\
04-14-69\end{array}$ & $\begin{array}{r}2,066 \\
2,064 \\
2,053 \\
-- \\
- \\
2,056\end{array}$ & $\begin{array}{l}\mathrm{V} \\
\mathrm{V} \\
\mathrm{V} \\
- \\
\mathrm{R}\end{array}$ & $\begin{array}{l}\mathrm{R} \\
\mathrm{R} \\
- \\
\mathrm{P} \\
\mathrm{P} \\
-\end{array}$ \\
\hline
\end{tabular}


Table 2. Depth to water in ground-water supply wells at the Nevada Test Site (NTS), Nye County, Nevada, calendar years 1951-91-Continued

\begin{tabular}{|c|c|c|c|c|c|c|c|c|c|c|c|c|c|}
\hline \multirow{2}{*}{$\begin{array}{c}\text { NTS } \\
\text { hole } \\
\text { number }\end{array}$} & \multirow{2}{*}{$\begin{array}{l}\text { USGS standard } \\
\text { Identification }\end{array}$} & \multirow{2}{*}{$\begin{array}{c}\text { Latitude } \\
\text { (degrees, } \\
\text { minutes, } \\
\text { seconds) }\end{array}$} & \multirow{2}{*}{$\begin{array}{l}\text { Longitude } \\
\text { (degrees, } \\
\text { minutes, } \\
\text { seconds) }\end{array}$} & \multirow{2}{*}{$\begin{array}{l}\text { Date well } \\
\text { completed } \\
\text { (month, } \\
\text { day, year) }\end{array}$} & \multirow{2}{*}{$\begin{array}{l}\text { Land- } \\
\text { surface } \\
\text { altitude } \\
\text { (feet) }\end{array}$} & \multirow{2}{*}{$\begin{array}{l}\text { Well } \\
\text { depth } \\
\text { (feet) }\end{array}$} & \multicolumn{3}{|c|}{$\begin{array}{c}\text { Depth of } \\
\text { open interval }\end{array}$} & \multicolumn{4}{|c|}{ Measurement } \\
\hline & & & & & & & $\begin{array}{c}\text { Top } \\
\text { (feet) }\end{array}$ & $\begin{array}{c}\text { Bottom } \\
\text { (feet) }\end{array}$ & Type & $\begin{array}{c}\text { Date } \\
\text { (month, } \\
\text { day, year) }\end{array}$ & $\begin{array}{l}\text { Depth to } \\
\text { water } \\
\text { (feet) }\end{array}$ & Method & $\begin{array}{l}\text { Site } \\
\text { status }\end{array}$ \\
\hline \multicolumn{14}{|c|}{ AREA 3} \\
\hline WW-A $A^{1,2}$ & 370142116021100 & 370213 & 1160210 & $08-28-60$ & 4,006 & 1,730 & $\begin{array}{l}1,555 \\
1,600\end{array}$ & $\begin{array}{l}1,600 \\
1,730\end{array}$ & $\begin{array}{l}\mathrm{X} \\
\mathrm{X}\end{array}$ & $08-28-60$ & 1,604 & $\mathbf{R}$ & - \\
\hline \multirow[t]{4}{*}{ WW-A ${ }^{1,2}$} & 370142116021101 & 370213 & 1160210 & $09-05-60$ & 4,006 & 1,870 & $\begin{array}{l}1,608 \\
1,555 \\
1,600\end{array}$ & $\begin{array}{l}1,870 \\
1,600 \\
1,870\end{array}$ & $\begin{array}{l}\mathrm{P} \\
\mathrm{X} \\
\mathrm{X}\end{array}$ & $\begin{array}{l}09-20-60 \\
11-30-60 \\
01-06-61 \\
01-13-61 \\
06-20-61\end{array}$ & $\begin{array}{l}1,604 \\
1,602 \\
1,602 \\
1,602 \\
1,604\end{array}$ & $\begin{array}{l}R \\
V \\
V \\
V \\
V\end{array}$ & $\begin{array}{l}- \\
- \\
- \\
-\end{array}$ \\
\hline & & & & & & & & & & $\begin{array}{l}10-04-61 \\
11-01-61 \\
11-02-61 \\
02-07-63 \\
06-07-63\end{array}$ & $\begin{array}{r}-- \\
\overline{-} \\
1,607\end{array}$ & $\begin{array}{l}- \\
\dot{v} \\
-\end{array}$ & $\begin{array}{l}\mathrm{P} \\
\mathrm{P} \\
\mathrm{P} \\
- \\
\mathrm{P}\end{array}$ \\
\hline & & & & & & & & & & $\begin{array}{l}08-19-63 \\
09-07-63 \\
09-08-63 \\
04-10-69 \\
03-23-71 \\
11-05-71\end{array}$ & $\begin{array}{l}1,614 \\
1,614 \\
1,611 \\
1,611 \\
1,616 \\
1,617\end{array}$ & $\begin{array}{l}\text { V } \\
\text { V } \\
\text { V } \\
R \\
\text { V } \\
\text { V }\end{array}$ & $\begin{array}{l}- \\
- \\
- \\
\dot{R} \\
\mathrm{R}\end{array}$ \\
\hline & \multicolumn{13}{|c|}{ AREA 5} \\
\hline RNM-2S & 364922115580101 & 364921 & 1155801 & $04-01-74$ & 3,133 & 1,120 & $\begin{array}{l}1,038 \\
1,120\end{array}$ & $\begin{array}{l}1,119 \\
1,156\end{array}$ & $\stackrel{P}{X}$ & $\begin{array}{l}08-02-74 \\
03-21-80 \\
06-13-80 \\
09-27-90 \\
10-03-90\end{array}$ & $\begin{array}{l}720 \\
725 \\
726 \\
728 \\
728\end{array}$ & $\begin{array}{l}V \\
V \\
V \\
V \\
V\end{array}$ & $\begin{array}{l}- \\
- \\
- \\
.\end{array}$ \\
\hline & & & & & & & & & & $\begin{array}{l}10-12-90 \\
10-19-90 \\
10-26-90 \\
11-02-90 \\
11-15-90\end{array}$ & $\begin{array}{l}728 \\
728 \\
728 \\
726 \\
726\end{array}$ & $\begin{array}{l}V \\
V \\
V \\
T \\
V\end{array}$ & $\begin{array}{l}- \\
- \\
- \\
-\end{array}$ \\
\hline & & & & & & & & & & $\begin{array}{l}12-12-90 \\
01-10-91 \\
01-16-91 \\
01-24-91 \\
02-05-91\end{array}$ & $\begin{array}{r}738 \\
727 \\
726 \\
--\end{array}$ & $\begin{array}{l}- \\
\mathrm{T} \\
\mathrm{T} \\
\mathrm{T} \\
-\end{array}$ & $\begin{array}{l}\mathrm{P} \\
- \\
\dot{P}\end{array}$ \\
\hline & & & & & & & & & & $\begin{array}{l}02-21-91 \\
04-11-91 \\
04-26-91 \\
05-06-91 \\
05-17-91\end{array}$ & $\begin{array}{r}- \\
- \\
\overline{-} \\
\overline{-} \\
-\end{array}$ & $\begin{array}{l}- \\
- \\
\bar{T} \\
-\end{array}$ & $\begin{array}{l}\mathrm{P} \\
\mathrm{P} \\
\mathrm{P} \\
\mathrm{R} \\
\mathrm{P}\end{array}$ \\
\hline & & & & & & & & & & $\begin{array}{l}05-29-91 \\
06-25-91 \\
07-09-91 \\
08-16-91 \\
08-28-91 \\
09-27-91\end{array}$ & $\begin{array}{l}-- \\
-- \\
-- \\
- \\
- \\
727\end{array}$ & $\begin{array}{l}- \\
- \\
- \\
\end{array}$ & $\begin{array}{l}P \\
P \\
P \\
P \\
P \\
-\end{array}$ \\
\hline
\end{tabular}


Table 2. Depth to water in ground-water supply wells at the Nevada Test Site (NTS), Nye County, Nevada, calendar years 1951-91--Continued

\begin{tabular}{|c|c|c|c|c|c|c|c|c|c|c|c|c|c|}
\hline \multirow{2}{*}{$\begin{array}{c}\text { NTS } \\
\text { hole } \\
\text { number }\end{array}$} & \multirow{2}{*}{$\begin{array}{l}\text { USGS standard } \\
\text { identification }\end{array}$} & \multirow{2}{*}{$\begin{array}{c}\text { Latitude } \\
\text { (degrees, } \\
\text { minutes, } \\
\text { seconds) }\end{array}$} & \multirow{2}{*}{$\begin{array}{l}\text { Longitude } \\
\text { (degrees, } \\
\text { minutes, } \\
\text { seconds) }\end{array}$} & \multirow{2}{*}{$\begin{array}{l}\text { Date well } \\
\text { completed } \\
\text { (month, } \\
\text { day, year) }\end{array}$} & \multirow{2}{*}{$\begin{array}{l}\text { Land- } \\
\text { surface } \\
\text { altitude } \\
\text { (feet) }\end{array}$} & \multirow{2}{*}{$\begin{array}{l}\text { Well } \\
\text { depth } \\
\text { (feet) }\end{array}$} & \multicolumn{3}{|c|}{$\begin{array}{c}\text { Depth of } \\
\text { open interval }\end{array}$} & \multicolumn{4}{|c|}{ Measurement } \\
\hline & & & & & & & $\begin{array}{l}\text { Top } \\
\text { (feet) }\end{array}$ & $\begin{array}{c}\text { Bottom } \\
\text { (feet) }\end{array}$ & Type & $\begin{array}{c}\text { Date } \\
\text { (month, } \\
\text { day, year) }\end{array}$ & $\begin{array}{l}\text { Depth to } \\
\text { water } \\
\text { (feet) }\end{array}$ & Method & $\begin{array}{l}\text { Site } \\
\text { status }\end{array}$ \\
\hline UE-5c WW ${ }^{1}$ & 365011115584701 & 365011 & 1155847 & $11-03-64$ & 3,216 & 2,682 & $\begin{array}{l}1,682 \\
2,385\end{array}$ & $\begin{array}{l}2,385 \\
2,682\end{array}$ & $\begin{array}{l}\mathrm{X} \\
\mathrm{X}\end{array}$ & $02-17-66$ & 825 & $\mathrm{R}$ & - \\
\hline UE-5c WW ${ }^{1}$ & 365011115584702 & 365011 & 1155847 & $04-21-66$ & 3,216 & 2,682 & $\begin{array}{l}1,100 \\
1,682 \\
2,385\end{array}$ & $\begin{array}{l}1,300 \\
2,385 \\
2,682\end{array}$ & $\begin{array}{l}\mathrm{P} \\
\mathrm{X} \\
\mathrm{X}\end{array}$ & $\begin{array}{l}03-20-71 \\
03-23-71 \\
10-27-71 \\
07-30-72 \\
01-18-73 \\
08-11-87\end{array}$ & $\begin{array}{l}807 \\
805 \\
807 \\
806 \\
807 \\
810\end{array}$ & $\begin{array}{l}\text { V } \\
\text { V } \\
\text { V } \\
\text { V } \\
\text { V } \\
\text { V }\end{array}$ & $\begin{array}{l}- \\
: \\
-\end{array}$ \\
\hline WW-5a & 364635115572901 & 364635 & 1155729 & $03-23-51$ & 3,093 & 887 & $\begin{array}{l}642 \\
877\end{array}$ & $\begin{array}{l}877 \\
910\end{array}$ & $\stackrel{p}{\mathrm{X}}$ & $\begin{array}{c}03--51 \\
01-26-59 \\
09-14-59 \\
09-23-59 \\
09-24-59\end{array}$ & $\begin{array}{l}699 \\
698 \\
696 \\
696 \\
697\end{array}$ & $\begin{array}{l}R \\
A \\
S \\
S \\
A\end{array}$ & $\begin{array}{l}- \\
- \\
-\end{array}$ \\
\hline & & & & & & & & & & $\begin{array}{l}10-07-59 \\
11-04-71 \\
07-27-72 \\
01-16-80\end{array}$ & $\begin{array}{l}696 \\
707 \\
705 \\
709\end{array}$ & $\begin{array}{l}S \\
V \\
S \\
V\end{array}$ & $\begin{array}{l}- \\
- \\
-\end{array}$ \\
\hline Ww-5b & 364805115580801 & 364805 & 1155808 & $05-07-51$ & 3,092 & 900 & 700 & 900 & $\mathbf{P}$ & $\begin{array}{l}08-25-59 \\
08-25-59 \\
08-26-59 \\
08-27-59 \\
08-27-59\end{array}$ & $\begin{array}{l}683 \\
683 \\
683 \\
683 \\
687\end{array}$ & $\begin{array}{l}\text { V } \\
\text { V } \\
\text { S } \\
\text { S } \\
\text { A }\end{array}$ & $\begin{array}{l}- \\
- \\
\dot{R}\end{array}$ \\
\hline & & & & & & & & & & $\begin{array}{l}08-29-59 \\
08-31-59 \\
12-23-60 \\
04-12-62 \\
06-07-62\end{array}$ & $\begin{array}{r}684 \\
683 \\
683 \\
-- \\
681\end{array}$ & $\begin{array}{l}S \\
S \\
S \\
- \\
V\end{array}$ & $\begin{array}{l}- \\
\dot{P} \\
-\end{array}$ \\
\hline & & & & & & & & & & $\begin{array}{l}10-19-64 \\
03-21-69 \\
03-25-71 \\
10-29-71 \\
07-30-72\end{array}$ & $\begin{array}{l}684 \\
682 \\
683 \\
683 \\
683\end{array}$ & $\begin{array}{l}R \\
R \\
V \\
V \\
S\end{array}$ & $\begin{array}{l}- \\
- \\
- \\
-\end{array}$ \\
\hline & & & & & & & & & & $\begin{array}{l}01-16-80 \\
05-06-91\end{array}$ & $\begin{array}{l}687 \\
689\end{array}$ & $\begin{array}{l}V \\
T\end{array}$ & - \\
\hline WW-5c & 364708115574401 & 364720 & 1155749 & $03-24-54$ & 3,081 & 1,200 & $\begin{array}{r}887 \\
1,187\end{array}$ & $\begin{array}{l}1,187 \\
1,200\end{array}$ & $\begin{array}{l}\mathrm{P} \\
\mathrm{X}\end{array}$ & $\begin{array}{l}04-26-54 \\
04-03-59 \\
09-09-59 \\
09-14-59 \\
12-13-60\end{array}$ & $\begin{array}{l}693 \\
694 \\
692 \\
692 \\
700\end{array}$ & $\begin{array}{l}A \\
S \\
S \\
S \\
S\end{array}$ & $\begin{array}{l}- \\
- \\
-\end{array}$ \\
\hline
\end{tabular}


Table 2. Depth to water in ground-water supply wells at the Nevada Test Site (NTS), Nye County, Nevada, calendar years 1951-91-Continued

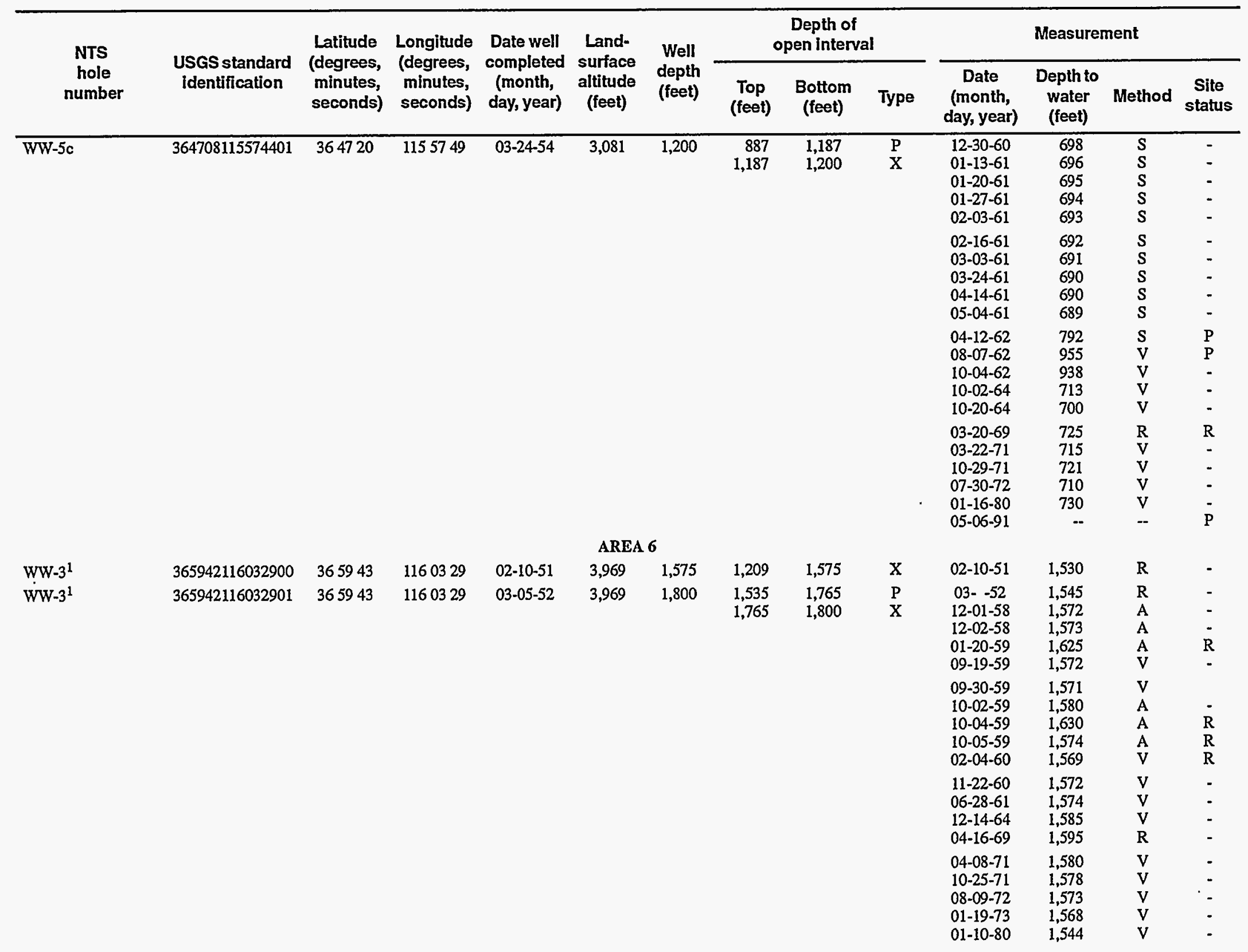


Table 2. Depth to water in ground-water supply wells at the Nevada Test Site (NTS), Nye County, Nevada, calendar years 1951-91--Continued

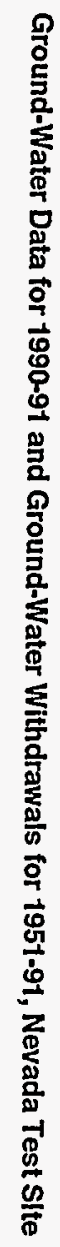

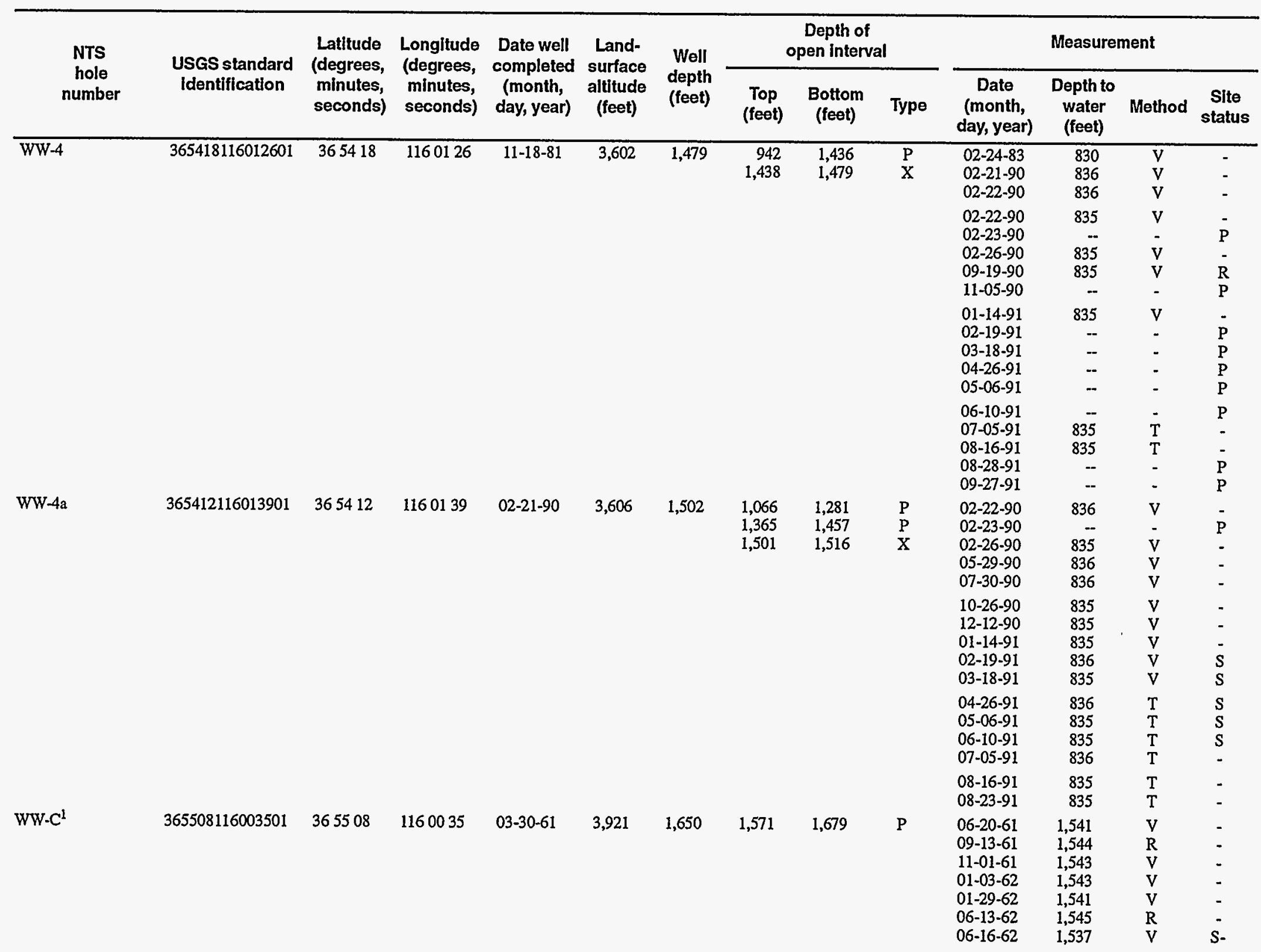


Table 2. Depth to water in ground-water supply wells at the Nevada Test Site (NTS), Nye County, Nevada, calendar years 1951-91-Continued

\begin{tabular}{|c|c|c|c|c|c|c|c|c|c|c|c|c|c|}
\hline \multirow{2}{*}{$\begin{array}{c}\text { NTS } \\
\text { hole } \\
\text { number }\end{array}$} & \multirow{2}{*}{$\begin{array}{l}\text { USGS standard } \\
\text { Identification }\end{array}$} & \multirow{2}{*}{$\begin{array}{c}\text { Latitude } \\
\text { (degrees, } \\
\text { minutes, } \\
\text { seconds) }\end{array}$} & \multirow{2}{*}{$\begin{array}{c}\text { Longitude } \\
\text { (degrees, } \\
\text { minutes, } \\
\text { seconds) }\end{array}$} & \multirow{2}{*}{$\begin{array}{l}\text { Date well } \\
\text { completed } \\
\text { (month, } \\
\text { day, year) }\end{array}$} & \multirow{2}{*}{$\begin{array}{l}\text { Land- } \\
\text { surface } \\
\text { altitude } \\
\text { (feet) }\end{array}$} & \multirow{2}{*}{$\begin{array}{l}\text { Well } \\
\text { depth } \\
\text { (feet) }\end{array}$} & \multicolumn{3}{|c|}{$\begin{array}{c}\text { Depth of } \\
\text { open interval }\end{array}$} & \multicolumn{4}{|c|}{ Measurement } \\
\hline & & & & & & & $\begin{array}{c}\text { Top } \\
\text { (feet) }\end{array}$ & $\begin{array}{c}\text { Bottom } \\
\text { (feet) }\end{array}$ & Type & $\begin{array}{c}\text { Date } \\
\text { (month, } \\
\text { day, year) }\end{array}$ & $\begin{array}{c}\text { Depth to } \\
\text { water } \\
\text { (feet) }\end{array}$ & Method & $\begin{array}{l}\text { Site } \\
\text { status }\end{array}$ \\
\hline WW-C ${ }^{1}$ & 365508116003502 & 365508 & 1160035 & $04-30-67$ & 3,921 & 1,624 & $\begin{array}{l}1,560 \\
1,624\end{array}$ & $\begin{array}{l}1,624 \\
1,701\end{array}$ & $\begin{array}{l}\mathrm{P} \\
\mathrm{X}\end{array}$ & $\begin{array}{c}04-11-69 \\
03-29-71 \\
10-25-71 \\
08-06-72 \\
03-18-73 \\
10-18-75\end{array}$ & $\begin{array}{l}1,542 \\
1,544 \\
1,545 \\
1,545 \\
1,544 \\
1,544\end{array}$ & $\begin{array}{l}R \\
V \\
V \\
V \\
V \\
L\end{array}$ & $\begin{array}{l}- \\
- \\
- \\
-\end{array}$ \\
\hline \multirow[t]{4}{*}{$W W-C-1^{3}$} & 365500116003901 & 365500 & 1160039 & $06-09-62$ & 3,921 & 1,707 & $\begin{array}{l}1,536 \\
1,650\end{array}$ & $\begin{array}{l}1,650 \\
1,707\end{array}$ & $\begin{array}{l}\mathrm{P} \\
\mathrm{X}\end{array}$ & $\begin{array}{l}10-05-62 \\
11-05-62 \\
01-25-63 \\
06-06-63 \\
06-07-63\end{array}$ & $\begin{array}{l}1,548 \\
1,547 \\
1,544 \\
1,544 \\
1,544\end{array}$ & $\begin{array}{l}R \\
R \\
V \\
V \\
V\end{array}$ & $\begin{array}{l}- \\
- \\
-\end{array}$ \\
\hline & & & & & & & & & & $\begin{array}{l}08-20-63 \\
09-07-63 \\
09-08-63 \\
10-08-63 \\
10-08-63\end{array}$ & $\begin{array}{l}1,543 \\
1,543 \\
1,544 \\
1,542 \\
1,544\end{array}$ & $\begin{array}{l}\mathrm{V} \\
\mathrm{V} \\
\mathrm{V} \\
\mathrm{V} \\
\mathrm{V}\end{array}$ & $\begin{array}{l}- \\
- \\
-\end{array}$ \\
\hline & & & & & & & & & & $\begin{array}{l}10-10-63 \\
04-11-69 \\
03-29-71 \\
08-06-72\end{array}$ & $\begin{array}{l}1,543 \\
1,542 \\
1,545 \\
1,546\end{array}$ & $\begin{array}{l}\mathrm{V} \\
\mathrm{R} \\
\mathrm{V} \\
\mathrm{V}\end{array}$ & $\begin{array}{l}- \\
-\end{array}$ \\
\hline & & & & & \multicolumn{2}{|c|}{ AREA 15} & & & & & & & \\
\hline UE-15d WW $W^{1,3}$ & 371230116021500 & 371233 & 1160229 & $03-06-62$ & 4,586 & 6,001 & 1,735 & 6,001 & $\mathrm{X}$ & $\begin{array}{l}03-26-62 \\
03-27-62 \\
03-28-62\end{array}$ & $\begin{array}{l}673 \\
669 \\
668\end{array}$ & $\begin{array}{l}\mathrm{V} \\
\mathrm{R} \\
\mathrm{V}\end{array}$ & $\dot{\mathrm{R}}$ \\
\hline UE-15d WW ${ }^{1,3}$ & 371230116021501 & 371233 & 1160229 & $04-17-62$ & 4,586 & 6,001 & 5,400 & 6,001 & $\mathrm{x}$ & $\begin{array}{l}10-02-63 \\
12-03-63 \\
05-20-80 \\
01-13-83\end{array}$ & $\begin{array}{l}667 \\
675 \\
694 \\
686\end{array}$ & $\begin{array}{l}\mathrm{V} \\
\mathrm{V} \\
\mathrm{V} \\
\mathrm{v}\end{array}$ & :- \\
\hline \multicolumn{14}{|c|}{ AREA 16} \\
\hline UE-16d WW' & 370406116095600 & 370412 & 1160951 & $05-23-77$ & 4,684 & 830 & 80 & 830 & $\mathrm{x}^{\prime}$ & $05-23-77$ & 752 & $\mathrm{~V}$ & $\mathrm{Z}$ \\
\hline UE-16d WW & 370412116095101 & 370412 & 1160951 & $03-03-81$ & 4,684 & 2,020 & 1,145 & 1,310 & $P$ & $03-04-81$ & 753 & L & $\mathbf{R}$ \\
\hline \multicolumn{14}{|c|}{ AREA 18} \\
\hline \multirow[t]{2}{*}{ WW-8 } & 370956116172101 & 370956 & 1161721 & $01-07-63$ & 5,695 & 1,862 & $\begin{array}{l}1,250 \\
1,450 \\
1,630\end{array}$ & $\begin{array}{l}1,300 \\
1,500 \\
1,780\end{array}$ & $\begin{array}{l}\mathrm{P} \\
\mathrm{P} \\
\mathrm{P}\end{array}$ & $\begin{array}{l}01-09-63 \\
01-10-63 \\
03-21-71 \\
03-24-71 \\
10-25-71\end{array}$ & $\begin{array}{l}1,068 \\
1,068 \\
1,076 \\
1,076 \\
1,077\end{array}$ & $\begin{array}{l}V \\
V \\
V \\
V \\
V\end{array}$ & $\begin{array}{l}Z \\
Z \\
- \\
- \\
-\end{array}$ \\
\hline & & & & & & & & & & $\begin{array}{l}08-07-72 \\
06-29-78 \\
02-17-82\end{array}$ & $\begin{array}{l}1,078 \\
1,078 \\
1,076\end{array}$ & $\begin{array}{l}\text { V } \\
L \\
\text { V }\end{array}$ & - \\
\hline
\end{tabular}


Table 2. Depth to water in ground-water supply wells at the Nevada Test Site (NTS), Nye County, Nevada, calendar years 1951-91--Continued

\begin{tabular}{|c|c|c|c|c|c|c|c|c|c|c|c|c|c|}
\hline \multirow{2}{*}{$\begin{array}{c}\text { NTS } \\
\text { hole } \\
\text { number }\end{array}$} & \multirow{2}{*}{$\begin{array}{l}\text { USGS standard } \\
\text { identification }\end{array}$} & \multirow{2}{*}{$\begin{array}{l}\text { Latitude } \\
\text { (degrees, } \\
\text { minutes, } \\
\text { seconds) }\end{array}$} & \multirow{2}{*}{$\begin{array}{l}\text { Longitude } \\
\text { (degrees, } \\
\text { minutes, } \\
\text { seconds) }\end{array}$} & \multirow{2}{*}{$\begin{array}{l}\text { Date well } \\
\text { completed } \\
\text { (month, } \\
\text { day, year) }\end{array}$} & \multirow{2}{*}{$\begin{array}{l}\text { Land- } \\
\text { surface } \\
\text { altitude } \\
\text { (feet) }\end{array}$} & \multirow{2}{*}{$\begin{array}{l}\text { Well } \\
\text { depth } \\
\text { (feet) }\end{array}$} & \multicolumn{3}{|c|}{$\begin{array}{c}\text { Depth of } \\
\text { open interval }\end{array}$} & \multicolumn{4}{|c|}{ Measurement } \\
\hline & & & & & & & $\begin{array}{l}\text { Top } \\
\text { (feet) }\end{array}$ & $\begin{array}{c}\text { Bottom } \\
\text { (feet) }\end{array}$ & Type & $\begin{array}{c}\text { Date } \\
\text { (month, } \\
\text { day, year) }\end{array}$ & $\begin{array}{l}\text { Depth to } \\
\text { water } \\
\text { (feet) }\end{array}$ & Method & $\begin{array}{l}\text { Site } \\
\text { status }\end{array}$ \\
\hline \multicolumn{14}{|c|}{ AREA 19} \\
\hline UE-19c WW 1 & 371608116191001 & 371608 & 1161910 & $04-29-64$ & 7,033 & 4,520 & $\begin{array}{l}2,421 \\
2,604\end{array}$ & $\begin{array}{l}2,604 \\
4,520\end{array}$ & $\begin{array}{l}\mathrm{X} \\
\mathrm{X}\end{array}$ & $04-30-64$ & 2,345 & V & - \\
\hline \multirow[t]{2}{*}{ UE-19c WW' } & 371608116191002 & 371608 & 1161910 & $06-30-75$ & 7,033 & 8,489 & $\begin{array}{l}2,421 \\
3,209\end{array}$ & $\begin{array}{l}3,209 \\
8,489\end{array}$ & $\begin{array}{l}\mathrm{x} \\
\mathrm{x}\end{array}$ & $\begin{array}{l}12-23-74 \\
06-26-75 \\
07-28-75 \\
01-05-76 \\
01-22-76\end{array}$ & $\begin{array}{l}2,337 \\
2,315 \\
2,347 \\
2,314 \\
2,314\end{array}$ & $\begin{array}{l}\text { L } \\
\text { L } \\
\text { L } \\
\text { L } \\
\text { L }\end{array}$ & $\begin{array}{l}- \\
\\
\\
-\end{array}$ \\
\hline & & & & & & & & & & $\begin{array}{l}03-15-76 \\
12-12-84 \\
10-15-85\end{array}$ & $\begin{array}{l}2,333 \\
2,344 \\
2,336\end{array}$ & $\begin{array}{l}\text { L } \\
\text { V } \\
\text { V }\end{array}$ & $\begin{array}{l}- \\
-\end{array}$ \\
\hline UE-19e WW & 371750116195901 & 371750 & 1161959 & $08-22-64$ & 6,919 & 6,005 & 2,475 & 6,005 & $\mathrm{x}$ & $\begin{array}{l}09-03-64 \\
01-13-65 \\
04-21-65 \\
06-26-75\end{array}$ & $\begin{array}{l}2,218 \\
2,217 \\
2,218 \\
2,228\end{array}$ & $\begin{array}{l}\text { V } \\
\text { V } \\
R \\
L\end{array}$ & : \\
\hline UE-19gS WW & 371830116215300 & 371830 & 1162153 & $03-22-65$ & 6,719 & 4,508 & 2,650 & 4,508 & $\mathrm{X}$ & $03-24-65$ & 2,043 & $\mathrm{R}$ & - \\
\hline UE-19gS WW ${ }^{1}$ & 371830116215301 & 371830 & 1162153 & $05-04-65$ & 6,719 & 7,506 & $\begin{array}{l}4,349 \\
4,508\end{array}$ & $\begin{array}{l}4,508 \\
7,500\end{array}$ & $\underset{\mathrm{X}}{\mathrm{X}}$ & $05-06-65$ & 2,045 & $\mathrm{R}$ & - \\
\hline UE-19gS WW ${ }^{1,4}$ & 371830116215303 & 371830 & 1162153 & $05-09-65$ & 6,719 & 7,506 & $\begin{array}{l}2,650 \\
4,350 \\
4,508\end{array}$ & $\begin{array}{l}4,113 \\
4,508 \\
7,500\end{array}$ & $\begin{array}{l}\mathrm{X} \\
\mathrm{X} \\
\mathrm{X}\end{array}$ & $\begin{array}{l}03-20-71 \\
01-13-76\end{array}$ & $\begin{array}{l}2,044 \\
2,032\end{array}$ & $\begin{array}{l}\text { V } \\
\text { L }\end{array}$ & - \\
\hline \multicolumn{14}{|c|}{ AREA 20} \\
\hline$U-20 \mathrm{WW}$ & 371505116254501 & 371505 & 1162545 & $07-22-85$ & 6,468 & 3,268 & $\begin{array}{l}2,271 \\
3,199\end{array}$ & $\begin{array}{l}3,035 \\
3,268\end{array}$ & $\begin{array}{l}\mathrm{p} \\
\mathrm{X}\end{array}$ & $\begin{array}{l}07-25-85 \\
07-22-91 \\
08-20-91 \\
09-09-91 \\
09-23-91\end{array}$ & $\begin{array}{l}2,034 \\
2,058 \\
2,057 \\
2,057 \\
2,057\end{array}$ & $\begin{array}{l}\mathrm{V} \\
\mathrm{V} \\
\mathrm{V} \\
\mathrm{V} \\
\mathrm{V}\end{array}$ & $\begin{array}{l}: \\
:\end{array}$ \\
\hline $\mathrm{U}-20 \mathrm{a} 2 \mathrm{WW}^{\mathrm{l}}$ & 371434116251601 & 371434 & 1162516 & $02-17-64$ & 6,472 & 4,500 & $\begin{array}{l}860 \\
896\end{array}$ & $\begin{array}{r}896 \\
4,500\end{array}$ & $\begin{array}{l}\mathrm{X} \\
\mathrm{X}\end{array}$ & $03-30-64$ & 2,065 & $\mathrm{R}$ & - \\
\hline $\mathrm{U}-20 \mathrm{a} 2 \mathrm{WW}^{1}$ & 371434116251602 & 371434 & 1162516 & $06-30-64$ & 6,472 & 4,500 & 2,353 & 4,500 & $\mathrm{X}$ & $02-11-65$ & 2,066 & $\mathrm{R}$ & - \\
\hline $\mathrm{U}-20 \mathrm{a} 2 \mathrm{WW}^{1}$ & 371434116251603 & 371434 & 1162516 & $04-14-66$ & 6,472 & 4,500 & 2,356 & 4,500 & $\mathrm{x}$ & $10-23-75$ & 2,058 & $\mathrm{~L}$ & - \\
\hline \multicolumn{14}{|c|}{ AREA 22} \\
\hline Army $1 \mathrm{WW}$ & 363530116021401 & 363530 & 1160214 & $07-15-62$ & 3,154 & 1,953 & $\begin{array}{r}800 \\
1,368 \\
1,370 \\
1,684\end{array}$ & $\begin{array}{l}1,050 \\
1,370 \\
1,684 \\
1,953\end{array}$ & $\begin{array}{l}P \\
X \\
X \\
X\end{array}$ & $\begin{array}{l}07-17-62 \\
07-17-62 \\
09-11-62 \\
01-25-63 \\
11-07-63\end{array}$ & $\begin{array}{l}786 \\
785 \\
784 \\
784 \\
785\end{array}$ & $\begin{array}{l}\mathrm{V} \\
\mathrm{V} \\
\mathrm{V} \\
\mathrm{V} \\
\mathrm{V}\end{array}$ & $\begin{array}{l}Z \\
R \\
- \\
- \\
-\end{array}$ \\
\hline & & & & & & & & & & $\begin{array}{l}04-22-69 \\
03-18-71 \\
10-26-71 \\
08-12-72 \\
10-15-87\end{array}$ & $\begin{array}{l}787 \\
788 \\
787 \\
791 \\
784\end{array}$ & $\begin{array}{l}R \\
V \\
V \\
V \\
V\end{array}$ & $\begin{array}{l}- \\
R \\
R \\
R \\
-\end{array}$ \\
\hline
\end{tabular}


Table 2. Depth to water in ground-water supply wells at the Nevada Test Site (NTS), Nye County, Nevada, calendar years 1951-91-Continued

\begin{tabular}{|c|c|c|c|c|c|c|c|c|c|c|c|c|c|}
\hline \multirow{2}{*}{$\begin{array}{c}\text { NTS } \\
\text { hole } \\
\text { number }\end{array}$} & \multirow{2}{*}{$\begin{array}{l}\text { USGS standard } \\
\text { identification }\end{array}$} & \multirow{2}{*}{$\begin{array}{c}\text { Latitude } \\
\text { (degrees, } \\
\text { minutes, } \\
\text { seconds) }\end{array}$} & \multirow{2}{*}{$\begin{array}{l}\text { Longitude } \\
\text { (degrees, } \\
\text { minutes, } \\
\text { seconds) }\end{array}$} & \multirow{2}{*}{$\begin{array}{l}\text { Date well } \\
\text { completed } \\
\text { (month, } \\
\text { day, year) }\end{array}$} & \multirow{2}{*}{$\begin{array}{l}\text { Land- } \\
\text { surface } \\
\text { altitude } \\
\text { (feet) }\end{array}$} & \multirow{2}{*}{$\begin{array}{c}\text { Well } \\
\text { depth } \\
\text { (feet) }\end{array}$} & \multicolumn{3}{|c|}{$\begin{array}{c}\text { Depth of } \\
\text { open interval }\end{array}$} & \multicolumn{4}{|c|}{ Measurement } \\
\hline & & & & & & & $\begin{array}{c}\text { Top } \\
\text { (feet) }\end{array}$ & $\begin{array}{c}\text { Bottom } \\
\text { (feet) }\end{array}$ & Type & $\begin{array}{c}\text { Date } \\
\text { (month, } \\
\text { day, year) }\end{array}$ & $\begin{array}{l}\text { Depth to } \\
\text { water } \\
\text { (feet) }\end{array}$ & Method & $\begin{array}{l}\text { Site } \\
\text { status }\end{array}$ \\
\hline \multicolumn{14}{|c|}{ AREA 25} \\
\hline \multirow[t]{7}{*}{ J-11 WW } & 364706116170601 & 364706 & 1161706 & $07-19-57$ & 3,443 & 1,327 & $\begin{array}{l}1,075 \\
1,242\end{array}$ & $\begin{array}{l}1,095 \\
1,298\end{array}$ & $\begin{array}{l}\mathrm{P} \\
\mathrm{P}\end{array}$ & $\begin{array}{l}03-15-61 \\
03-15-61 \\
03-22-61 \\
04-10-61 \\
08-16-62\end{array}$ & $\begin{array}{l}1,042 \\
1,042 \\
1,042 \\
1,041 \\
1,040\end{array}$ & $\begin{array}{l}V \\
V \\
V \\
V \\
V\end{array}$ & $\begin{array}{l}- \\
- \\
- \\
-\end{array}$ \\
\hline & & & & & & & & & & $\begin{array}{l}11-27-63 \\
02-19-64 \\
02-19-64 \\
07-16-64 \\
10-06-64\end{array}$ & $\begin{array}{l}1,041 \\
1,040 \\
1,040 \\
1,042 \\
1,041\end{array}$ & $\begin{array}{l}\text { V } \\
R \\
R \\
V \\
\text { V }\end{array}$ & $\begin{array}{l}- \\
- \\
- \\
-\end{array}$ \\
\hline & & & & & & & & & & $\begin{array}{l}11-04-71 \\
08-06-72 \\
01-23-73 \\
03-22-73 \\
02-12-90\end{array}$ & $\begin{array}{l}1,041 \\
1,040 \\
1,042 \\
1,041 \\
1,040\end{array}$ & $\begin{array}{l}V \\
V \\
V \\
V \\
S\end{array}$ & $\begin{array}{l}- \\
- \\
-\end{array}$ \\
\hline & & & & & & & & & & $\begin{array}{l}07-10-90 \\
07-20-90 \\
07-27-90 \\
07-31-90 \\
08-09-90\end{array}$ & $\begin{array}{l}1,041 \\
1,041 \\
1,041 \\
1,041 \\
1,041\end{array}$ & $\begin{array}{l}S \\
S \\
S \\
S \\
S\end{array}$ & $\begin{array}{l}- \\
- \\
-\end{array}$ \\
\hline & & & & & & & & & & $\begin{array}{l}08-15-90 \\
08-21-90 \\
08-27-90 \\
09-14-90 \\
10-19-90\end{array}$ & $\begin{array}{l}1,040 \\
1,041 \\
1,041 \\
1,041 \\
1,040\end{array}$ & $\begin{array}{l}S \\
S \\
S \\
S \\
S\end{array}$ & $\begin{array}{l}- \\
- \\
- \\
-\end{array}$ \\
\hline & & & & & & & & & & $\begin{array}{l}11-19-90 \\
12-13-90 \\
01-24-91 \\
02-28-91 \\
03-27-91\end{array}$ & $\begin{array}{l}1,040 \\
1,040 \\
1,040 \\
1,040 \\
1,040\end{array}$ & $\begin{array}{l}S \\
S \\
S \\
S \\
S\end{array}$ & $\begin{array}{l}- \\
- \\
-\end{array}$ \\
\hline & & & & & & & & & & $\begin{array}{l}04-19-91 \\
05-07-91 \\
06-10-91 \\
07-23-91 \\
08-22-91 \\
09-16-91\end{array}$ & $\begin{array}{l}1,041 \\
1,041 \\
1,041 \\
1,041 \\
1,041 \\
1,041\end{array}$ & $\begin{array}{l}S \\
S \\
S \\
S \\
S \\
S\end{array}$ & $\begin{array}{l}- \\
- \\
- \\
- \\
-\end{array}$ \\
\hline$J-12 W^{1}$ & 364554116232400 & 364554 & 1162324 & $10-09-57$ & 3,128 & 887 & 793 & 868 & $\mathrm{P}$ & $\begin{array}{l}10-31-57 \\
01-29-58 \\
01-27-60 \\
08-22-62 \\
07-25-68\end{array}$ & $\begin{array}{l}734 \\
739 \\
741 \\
744 \\
743\end{array}$ & $\begin{array}{l}\mathrm{R} \\
\mathrm{R} \\
\mathrm{R} \\
\mathrm{R} \\
\mathrm{R}\end{array}$ & $\begin{array}{l}- \\
- \\
- \\
-\end{array}$ \\
\hline
\end{tabular}


Table 2. Depth to water in ground-water supply wells at the Nevada Test Site (NTS), Nye County, Nevada, calendar years 1951-91--Continued

\begin{tabular}{|c|c|c|c|c|c|c|c|c|c|c|c|c|c|}
\hline \multirow{2}{*}{$\begin{array}{c}\text { NTS } \\
\text { hole } \\
\text { number }\end{array}$} & \multirow{2}{*}{$\begin{array}{l}\text { USGS standard } \\
\text { identification }\end{array}$} & \multirow{2}{*}{$\begin{array}{l}\text { Latitude } \\
\text { (degrees, } \\
\text { minutes, } \\
\text { seconds) }\end{array}$} & \multirow{2}{*}{$\begin{array}{l}\text { Longitude } \\
\text { (degrees, } \\
\text { minutes, } \\
\text { seconds) }\end{array}$} & \multirow{2}{*}{$\begin{array}{l}\text { Date well } \\
\text { completed } \\
\text { (month, } \\
\text { day, year) }\end{array}$} & \multirow{2}{*}{$\begin{array}{l}\text { Land- } \\
\text { surface } \\
\text { altitude } \\
\text { (feet) }\end{array}$} & \multirow{2}{*}{$\begin{array}{l}\text { Well } \\
\text { depth } \\
\text { (feet) }\end{array}$} & \multicolumn{3}{|c|}{$\begin{array}{c}\text { Depth of } \\
\text { open interval }\end{array}$} & \multicolumn{4}{|c|}{ Measurement } \\
\hline & & & & & & & $\begin{array}{l}\text { Top } \\
\text { (feet) }\end{array}$ & $\begin{array}{c}\text { Bottom } \\
\text { (feet) }\end{array}$ & Type & $\begin{array}{c}\text { Date } \\
\text { (month, } \\
\text { day, year) }\end{array}$ & $\begin{array}{l}\text { Depth to } \\
\text { water } \\
\text { (feet) }\end{array}$ & Method & $\begin{array}{l}\text { Site } \\
\text { status }\end{array}$ \\
\hline \multirow[t]{6}{*}{$J-12 W W^{i}$} & 364554116232401 & 364554 & 1162324 & $08--68$ & 3,128 & 1,139 & $\begin{array}{l}793 \\
887\end{array}$ & $\begin{array}{r}868 \\
1,139\end{array}$ & $\begin{array}{l}\mathrm{P} \\
\mathrm{X}\end{array}$ & $\begin{array}{l}08-24-68 \\
04-21-69 \\
03-26-71 \\
04-26-74 \\
04-27-74 \\
04-28-74\end{array}$ & $\begin{array}{l}742 \\
742 \\
742 \\
741 \\
742 \\
742\end{array}$ & $\begin{array}{l}\mathrm{R} \\
\mathrm{R} \\
\mathrm{T} \\
\mathrm{V} \\
\mathrm{V} \\
\mathrm{V}\end{array}$ & $\begin{array}{l}- \\
\vdots \\
\vdots\end{array}$ \\
\hline & & & & & & & & & & $\begin{array}{l}04-28-74 \\
04-29-74 \\
04-30-74 \\
04-30-74 \\
05-01-74 \\
05-01-74\end{array}$ & $\begin{array}{l}742 \\
742 \\
742 \\
742 \\
742 \\
742\end{array}$ & $\begin{array}{l}\mathrm{V} \\
\mathrm{V} \\
\mathrm{V} \\
\mathrm{V} \\
\mathrm{V} \\
\mathrm{V}\end{array}$ & $\begin{array}{l}\dot{\bar{R}} \\
\dot{\mathrm{R}} \\
\dot{\mathrm{R}}\end{array}$ \\
\hline & & & & & & & & & & $\begin{array}{l}05-28-80 \\
02-03-81 \\
12-05-83 \\
01-25-90 \\
02-08-90 \\
03-09-90\end{array}$ & $\begin{array}{l}740 \\
739 \\
742 \\
740 \\
740 \\
740\end{array}$ & $\begin{array}{l}\mathrm{V} \\
\mathrm{V} \\
\mathrm{V} \\
\mathrm{S} \\
\mathrm{S} \\
\mathrm{S}\end{array}$ & $\begin{array}{l}: \\
: \\
:\end{array}$ \\
\hline & & & & & & & & & & $\begin{array}{l}04-17-90 \\
05-30-90 \\
06-18-90 \\
07-10-90 \\
08-20-90\end{array}$ & $\begin{array}{l}740 \\
740 \\
740 \\
740 \\
740\end{array}$ & $\begin{array}{l}S \\
S \\
S \\
S \\
S\end{array}$ & $:$ \\
\hline & & & & & & & & & & $\begin{array}{l}09-14-90 \\
10-29-90 \\
11-27-90 \\
12-13-90 \\
02-25-91\end{array}$ & $\begin{array}{l}740 \\
740 \\
740 \\
740 \\
740\end{array}$ & $\begin{array}{l}S \\
S \\
S \\
S \\
S\end{array}$ & $\begin{array}{l}- \\
- \\
-\end{array}$ \\
\hline & & & & & & & & & & $\begin{array}{l}04-24-91 \\
05-14-91 \\
06-10-91 \\
07-23-91 \\
08-21-91 \\
09-12-91\end{array}$ & $\begin{array}{l}740 \\
740 \\
740 \\
740 \\
740 \\
740\end{array}$ & $\begin{array}{l}S \\
S \\
S \\
S \\
S \\
S\end{array}$ & $\begin{array}{l}- \\
- \\
-\end{array}$ \\
\hline $\mathrm{J}-13 \mathrm{ww}^{1}$ & 364828116234000 & 364828 & 1162340 & $12-27-62$ & 3,318 & 1,481 & $\begin{array}{r}996 \\
1,301\end{array}$ & $\begin{array}{l}1,301 \\
1,386\end{array}$ & $\begin{array}{l}\mathrm{P} \\
\mathrm{P}\end{array}$ & $\begin{array}{l}12-30-62 \\
01-01-63\end{array}$ & $\begin{array}{l}927 \\
927\end{array}$ & $\begin{array}{l}\mathrm{v} \\
\mathrm{v}\end{array}$ & $\dot{\mathrm{R}}$ \\
\hline \multirow[t]{2}{*}{$\mathrm{J}-13 \mathrm{w} \mathrm{w}^{1}$} & 364828116234001 & 364828 & 1162340 & $01-06-63$ & 3,318 & 3,488 & $\begin{array}{r}996 \\
1,301 \\
2,690 \\
3,385\end{array}$ & $\begin{array}{l}1,301 \\
1,386 \\
3,312 \\
3,488\end{array}$ & $\begin{array}{l}\mathrm{P} \\
\mathrm{P} \\
\mathrm{P} \\
\mathrm{X}\end{array}$ & $\begin{array}{l}02-04-63 \\
02-04-63 \\
11-27-63 \\
12-17-63 \\
12-19-63\end{array}$ & $\begin{array}{l}928 \\
928 \\
928 \\
928 \\
929\end{array}$ & $\begin{array}{l}\mathrm{V} \\
\mathrm{V} \\
\mathrm{V} \\
\mathrm{V} \\
\mathrm{V}\end{array}$ & $:$ \\
\hline & & & & & & & & & & $\begin{array}{l}02-04-64 \\
02-07-64 \\
02-18-64 \\
03-31-64 \\
03-11-67\end{array}$ & $\begin{array}{l}928 \\
928 \\
928 \\
925 \\
929\end{array}$ & $\begin{array}{l}\text { V } \\
\text { V } \\
R \\
V \\
V\end{array}$ & $\begin{array}{l}- \\
- \\
-\end{array}$ \\
\hline
\end{tabular}


Table 2. Depth to water in ground-water supply wells at the Nevada Test Site (NTS), Nye County, Nevada, calendar years 1951-91-Continued

\begin{tabular}{|c|c|c|c|c|c|c|c|c|c|c|c|c|c|}
\hline \multirow{2}{*}{$\begin{array}{c}\text { NTS } \\
\text { hole } \\
\text { number }\end{array}$} & \multirow{2}{*}{$\begin{array}{l}\text { USGS standard } \\
\text { identification }\end{array}$} & \multirow{2}{*}{$\begin{array}{c}\text { Latitude } \\
\text { (degrees, } \\
\text { minutes, } \\
\text { seconds) }\end{array}$} & \multirow{2}{*}{$\begin{array}{l}\text { Longitude } \\
\text { (degrees, } \\
\text { minutes, } \\
\text { seconds) }\end{array}$} & \multirow{2}{*}{$\begin{array}{l}\text { Date well } \\
\text { completed } \\
\text { (month, } \\
\text { day, year) }\end{array}$} & \multirow{2}{*}{$\begin{array}{l}\text { Land- } \\
\text { surface } \\
\text { altitude } \\
\text { (feet) }\end{array}$} & \multirow{2}{*}{$\begin{array}{l}\text { Well } \\
\text { depth } \\
\text { (feet) }\end{array}$} & \multicolumn{3}{|c|}{$\begin{array}{c}\text { Depth of } \\
\text { open interval }\end{array}$} & \multicolumn{4}{|c|}{ Measurement } \\
\hline & & & & & & & $\begin{array}{c}\text { Top } \\
\text { (feet) }\end{array}$ & $\begin{array}{l}\text { Bottom } \\
\text { (feet) }\end{array}$ & Type & $\begin{array}{c}\text { Date } \\
\text { (month, } \\
\text { day, year) }\end{array}$ & $\begin{array}{c}\text { Depth to } \\
\text { water } \\
\text { (feet) }\end{array}$ & Method & $\begin{array}{l}\text { Site } \\
\text { status }\end{array}$ \\
\hline \multirow[t]{8}{*}{$J-13 W^{1}$} & 364828116234001 & 364828 & 1162340 & $01-06-63$ & 3,318 & 3,488 & $\begin{array}{r}996 \\
1,301 \\
2,690 \\
3,385\end{array}$ & $\begin{array}{l}1,301 \\
1,386 \\
3,312 \\
3,488\end{array}$ & $\begin{array}{l}P \\
P \\
P \\
X\end{array}$ & $\begin{array}{l}04-21-69 \\
03-26-71 \\
08-20-80 \\
10-31-83 \\
09-11-86\end{array}$ & $\begin{array}{l}930 \\
928 \\
926 \\
929 \\
928\end{array}$ & $\begin{array}{l}\mathrm{R} \\
\mathrm{T} \\
\mathrm{V} \\
\mathrm{V} \\
\mathrm{S}\end{array}$ & $\begin{array}{l}- \\
- \\
- \\
-\end{array}$ \\
\hline & & & & & & & & & & $\begin{array}{l}03-24-87 \\
05-08-87 \\
12-09-87 \\
03-04-88 \\
07-07-88\end{array}$ & $\begin{array}{l}928 \\
928 \\
928 \\
928 \\
928\end{array}$ & $\begin{array}{l}S \\
S \\
S \\
S \\
S\end{array}$ & $\begin{array}{l}- \\
- \\
-\end{array}$ \\
\hline & & & & & & & & & & $\begin{array}{l}10-26-88 \\
01-18-89 \\
02-22-89 \\
03-22-89 \\
05-30-89\end{array}$ & $\begin{array}{l}928 \\
928 \\
928 \\
928 \\
928\end{array}$ & $\begin{array}{l}S \\
S \\
S \\
S \\
S\end{array}$ & $\begin{array}{l}- \\
- \\
- \\
-\end{array}$ \\
\hline & & & & & & & & & & $\begin{array}{l}07-21-89 \\
08-14-89 \\
11-21-89 \\
12-20-89 \\
01-25-90\end{array}$ & $\begin{array}{l}928 \\
928 \\
928 \\
928 \\
928\end{array}$ & $\begin{array}{l}S \\
S \\
S \\
S \\
S\end{array}$ & $\begin{array}{l}- \\
- \\
-\end{array}$ \\
\hline & & & & & & & & & & $\begin{array}{l}02-09-90 \\
03-09-90 \\
04-20-90 \\
05-23-90 \\
06-20-90\end{array}$ & $\begin{array}{l}928 \\
928 \\
928 \\
928 \\
928\end{array}$ & $\begin{array}{l}S \\
S \\
S \\
S \\
S\end{array}$ & $\begin{array}{l}- \\
- \\
-\end{array}$ \\
\hline & & & & & & & & & & $\begin{array}{l}07-10-90 \\
08-15-90 \\
09-14-90 \\
10-29-90 \\
11-27-90\end{array}$ & $\begin{array}{l}928 \\
928 \\
928 \\
928 \\
928\end{array}$ & $\begin{array}{l}S \\
S \\
S \\
S \\
S\end{array}$ & $\begin{array}{l}- \\
- \\
- \\
-\end{array}$ \\
\hline & & & & & & & & & & $\begin{array}{l}12-13-90 \\
01-31-91 \\
02-25-91 \\
03-27-91 \\
04-24-91\end{array}$ & $\begin{array}{l}928 \\
928 \\
928 \\
928 \\
927\end{array}$ & $\begin{array}{l}S \\
S \\
S \\
S \\
S\end{array}$ & $\begin{array}{l}- \\
- \\
-\end{array}$ \\
\hline & & & & & & & & & & $\begin{array}{l}05-16-91 \\
06-10-91 \\
07-25-91 \\
08-22-91 \\
09-16-91\end{array}$ & $\begin{array}{l}928 \\
928 \\
928 \\
928 \\
928\end{array}$ & $\begin{array}{l}S \\
S \\
S \\
S \\
S\end{array}$ & $\begin{array}{l}- \\
- \\
- \\
-\end{array}$ \\
\hline
\end{tabular}

${ }^{1}$ Repeated NTS hole numbers reflect more than one depth-to-water measurement interval.

2 Depth-to-water measurements corrected for borehole deviation from vertical; construction information not corrected for borehole deviation from vertical.

${ }^{3}$ Measurement not corrected for borehole deviation from vertical.

${ }^{4}$ Section of casing left in hole from 4,114 feet to 4,350 feet. 
§ Table 3. Annual withdrawals from ground-water supply wells at the Nevada Test Site (NTS), Nye County, Nevada, calendar years 1951-91

NTS hole number--Drill-hole number assigned by Raytheon Services Nevada; see section "Site Designations" in text.

USGS standard Identification--U.S. Geological Survey site designation; see section "Site Designations" in text.

Date well completed--Date that borehole construction work ceased; see section "Measurements" in text.

Land-surface altitude--Datum is sea level. Value may not represent current altitude; see section "Depth to Water" in text.

Well depth--Datum is land surface. Represents most recent available accessible depth, see section "Measurements" in text.

Depth of open intervals--Datum is land surface. Bottom of open interval may be deeper than present accessible well depth. Type: P, perforated or slotted casing; $X$, open (uncased) hole.

Primary water-yielding units--A, alluvium; $C$, carbonate rock; V, volcanic rock.

Ground-water subbasin--AFFCR, Alkali Flat-Fumace Creek Ranch; AM, Ash Meadows.

Ground-water withdrawals-Source: E, Reynolds Electrical and Engineering Company, Inc. (REECo) water production reports and includes estimated values; L, Los Alamos National Laboratory;

¿. M, REECo water production reports; R, Claassen (1973); Z, estimated average from Claassen (1973).

\begin{tabular}{|c|c|c|c|c|c|c|c|c|c|c|c|c|c|c|c|c|}
\hline \multirow{2}{*}{$\begin{array}{l}\text { NTS hole } \\
\text { number }\end{array}$} & \multirow{2}{*}{$\begin{array}{c}\text { USGS } \\
\text { standard } \\
\text { Identification }\end{array}$} & \multirow{2}{*}{$\begin{array}{l}\text { Latitude } \\
\text { (degrees, } \\
\text { minutes, } \\
\text { seconds) }\end{array}$} & \multirow{2}{*}{$\begin{array}{l}\text { Longitude } \\
\text { (degrees, } \\
\text { minutes, } \\
\text { seconds) }\end{array}$} & \multirow{2}{*}{$\begin{array}{c}\text { Date well } \\
\text { completed } \\
\text { (month, } \\
\text { day, year) }\end{array}$} & \multirow{2}{*}{$\begin{array}{l}\text { Land- } \\
\text { surface } \\
\text { altitude } \\
\text { (feet) }\end{array}$} & \multirow{2}{*}{$\begin{array}{l}\text { Well } \\
\text { depth } \\
\text { (feet) }\end{array}$} & \multicolumn{3}{|c|}{$\begin{array}{c}\text { Depth of } \\
\text { open interval }\end{array}$} & \multirow{2}{*}{$\begin{array}{l}\text { Primary } \\
\text { water- } \\
\text { yielding } \\
\text { units }\end{array}$} & \multirow{2}{*}{$\begin{array}{l}\text { Ground- } \\
\text { water } \\
\text { subbasin }\end{array}$} & \multicolumn{5}{|c|}{ Ground-water withdrawal } \\
\hline & & & & & & & $\begin{array}{c}\text { Top } \\
\text { (feet) }\end{array}$ & $\begin{array}{l}\text { Bottom } \\
\text { (feet) }\end{array}$ & Type & & & $\begin{array}{l}\text { Calendar } \\
\text { year }\end{array}$ & $\begin{array}{l}\text { Million } \\
\text { gallons }\end{array}$ & $\begin{array}{c}\text { Acre- } \\
\text { feet }\end{array}$ & Source & $\begin{array}{c}\text { Days } \\
\text { estimated }\end{array}$ \\
\hline \multicolumn{17}{|c|}{ AREA 1} \\
\hline \multirow[t]{3}{*}{ UE-1r WW } & 370142116033301 & 370142 & 1160333 & $01-16-84$ & 4,042 & 3,145 & $\begin{array}{l}2,319 \\
2,350\end{array}$ & $\begin{array}{l}2,350 \\
4,182\end{array}$ & $\begin{array}{l}X \\
X\end{array}$ & $\mathrm{~V}, \mathrm{C}$ & $\mathrm{AM}$ & $\begin{array}{l}1984 \\
1985 \\
1986 \\
1987 \\
1988\end{array}$ & $\begin{array}{l}1.9 \\
7.0 \\
4.4 \\
5.0 \\
4.7\end{array}$ & $\begin{array}{r}6 \\
21 \\
14 \\
15 \\
15\end{array}$ & $\begin{array}{l}\mathrm{E} \\
\mathrm{E} \\
\mathrm{M} \\
\mathrm{M} \\
\mathrm{M}\end{array}$ & $\begin{array}{r}8 \\
20\end{array}$ \\
\hline & & & & & & & & & & & & $\begin{array}{l}1989 \\
1990 \\
1991\end{array}$ & $\begin{array}{l}0.0 \\
0.0 \\
0.0\end{array}$ & $\begin{array}{l}0 \\
0 \\
0\end{array}$ & $\begin{array}{l}\mathrm{M} \\
\mathrm{M} \\
\mathrm{M}\end{array}$ & \\
\hline & \multicolumn{16}{|c|}{ AREA 2} \\
\hline$W W-2^{1}$ & 370958116051512 & 370958 & 1160515 & $03-11-62$ & 4,470 & 3,422 & $\begin{array}{l}2,700 \\
3,164\end{array}$ & $\begin{array}{l}2,950 \\
3,412\end{array}$ & $\begin{array}{l}\mathrm{P} \\
\mathrm{P}\end{array}$ & C & $\mathrm{AM}$ & $\begin{array}{l}1962 \\
1963 \\
1964 \\
1965 \\
1966\end{array}$ & $\begin{array}{r}9.7 \\
23.1 \\
24.2 \\
35.0 \\
46.7\end{array}$ & $\begin{array}{r}30 \\
71 \\
74 \\
107 \\
143\end{array}$ & $\begin{array}{l}R \\
R \\
R \\
R \\
R\end{array}$ & \\
\hline & & & & & & & & & & & & $\begin{array}{l}1967 \\
1968 \\
1969 \\
1970 \\
1971\end{array}$ & $\begin{array}{l}51.4 \\
51.7 \\
70.7 \\
57.3 \\
36.5\end{array}$ & $\begin{array}{l}158 \\
159 \\
217 \\
176 \\
112\end{array}$ & $\begin{array}{l}R \\
R \\
R \\
R \\
R\end{array}$ & \\
\hline & & & & & & & & & & & & $\begin{array}{l}1983 \\
1984 \\
1985 \\
1986 \\
1987\end{array}$ & $\begin{array}{l}43.4 \\
48.1 \\
18.5 \\
19.4 \\
42.6\end{array}$ & $\begin{array}{r}133 \\
148 \\
57 \\
60 \\
131\end{array}$ & $\begin{array}{l}\mathrm{M} \\
\mathrm{E} \\
\mathrm{E} \\
\mathrm{M} \\
\mathrm{M}\end{array}$ & $\begin{array}{r}8 \\
20\end{array}$ \\
\hline
\end{tabular}


Table 3. Annual withdrawals from ground-water supply wells at the Nevada Test Site (NTS), Nye County, Nevada, calendar years 1951-91--Continued

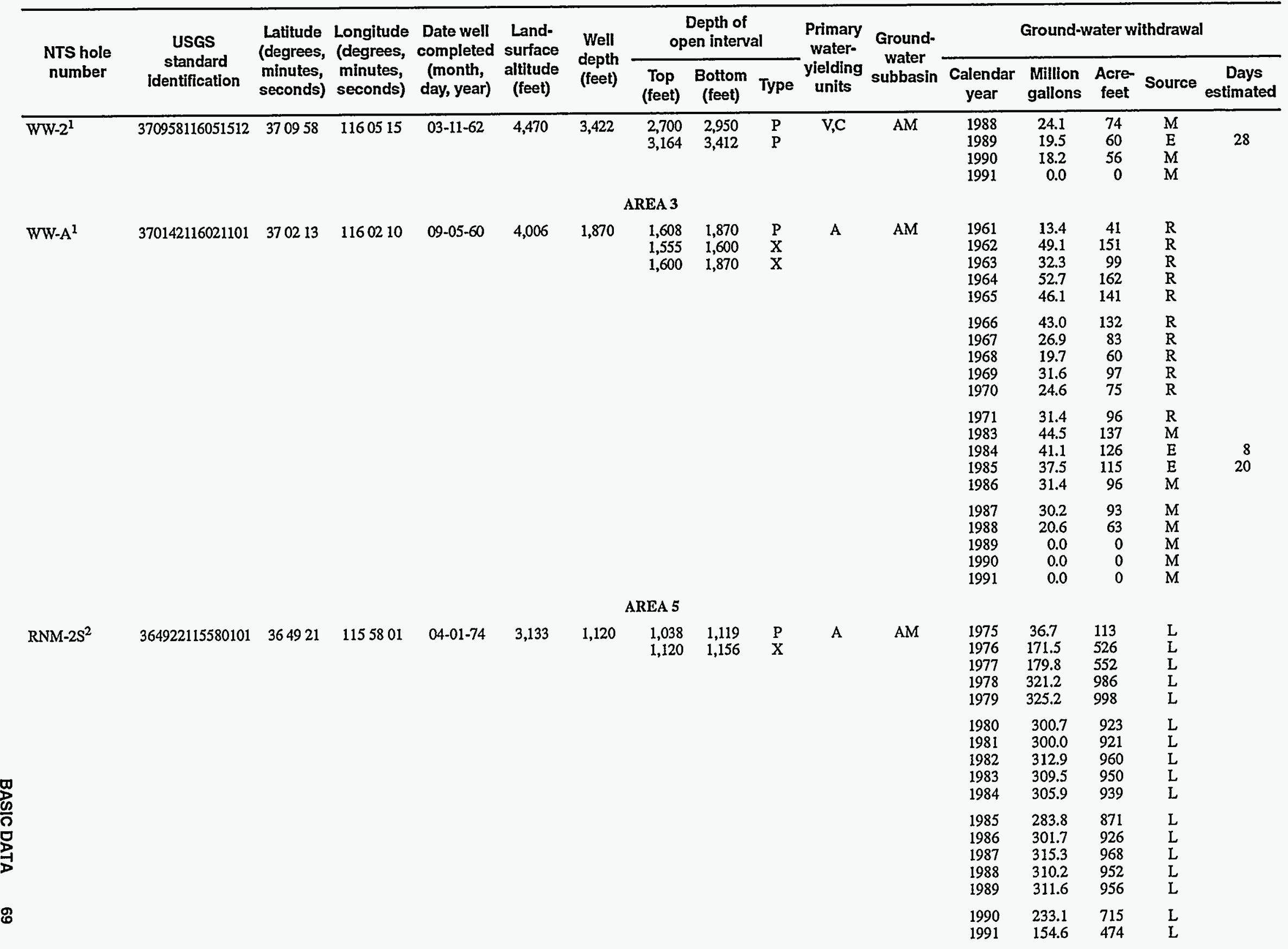


ò Table 3. Annual withdrawals from ground-water supply wells at the Nevada Test Site (NTS), Nye County, Nevada, calendar years 1951-91--Continued

\begin{tabular}{|c|c|c|c|c|c|c|c|c|c|c|c|c|c|c|c|c|}
\hline \multirow{2}{*}{$\begin{array}{l}\text { NTS hole } \\
\text { number }\end{array}$} & \multirow{2}{*}{$\begin{array}{c}\text { USGS } \\
\text { standard } \\
\text { Identification }\end{array}$} & \multirow{2}{*}{$\begin{array}{l}\text { Latitude } \\
\text { (degrees, } \\
\text { minutes, } \\
\text { seconds) }\end{array}$} & \multirow{2}{*}{$\begin{array}{l}\text { Longitude } \\
\text { (degrees, } \\
\text { minutes, } \\
\text { seconds) }\end{array}$} & \multirow{2}{*}{$\begin{array}{l}\text { Date well } \\
\text { completed } \\
\text { (month, } \\
\text { day, year) }\end{array}$} & \multirow{2}{*}{$\begin{array}{l}\text { Land- } \\
\text { surface } \\
\text { altitude } \\
\text { (feet) }\end{array}$} & \multirow{2}{*}{$\begin{array}{l}\text { Well } \\
\text { depth } \\
\text { (feet) }\end{array}$} & \multicolumn{3}{|c|}{$\begin{array}{l}\text { Depth of } \\
\text { open Interval }\end{array}$} & \multirow{2}{*}{$\begin{array}{c}\text { Primary } \\
\text { water- } \\
\text { yielding } \\
\text { units }\end{array}$} & \multirow{2}{*}{$\begin{array}{c}\text { Ground. } \\
\text { water } \\
\text { subbasin }\end{array}$} & \multicolumn{5}{|c|}{ Ground-water withdrawal } \\
\hline & & & & & & & $\begin{array}{c}\text { Top } \\
\text { (feet) }\end{array}$ & $\begin{array}{c}\text { Bottom } \\
\text { (feet) }\end{array}$ & Type & & & $\begin{array}{c}\text { Calendar } \\
\text { year }\end{array}$ & $\begin{array}{l}\text { Million } \\
\text { gallons }\end{array}$ & $\begin{array}{c}\text { Acre- } \\
\text { feet }\end{array}$ & Source & $\begin{array}{c}\text { Days } \\
\text { estimated }\end{array}$ \\
\hline \multirow[t]{2}{*}{ UE-5c WW } & 365011115584702 & 365011 & 1155847 & $04-21-66$ & 3,216 & 2,682 & $\begin{array}{l}1,100 \\
1,682 \\
2,385\end{array}$ & $\begin{array}{l}1,300 \\
2,385 \\
2,682\end{array}$ & $\begin{array}{l}\mathrm{P} \\
\mathrm{X} \\
\mathrm{X}\end{array}$ & $\mathrm{A}, \mathrm{V}$ & $\mathrm{AM}$ & $\begin{array}{l}1983 \\
1984 \\
1985 \\
1986 \\
1987\end{array}$ & $\begin{array}{l}1.3 \\
2.4 \\
5.0 \\
3.5 \\
8.4\end{array}$ & $\begin{array}{r}4 \\
7 \\
15 \\
11 \\
26\end{array}$ & $\begin{array}{l}\mathrm{M} \\
\mathrm{E} \\
\mathrm{E} \\
\mathrm{M} \\
\mathrm{M}\end{array}$ & $\begin{array}{r}8 \\
20\end{array}$ \\
\hline & & & & & & & & & & & & $\begin{array}{l}1988 \\
1989 \\
1990 \\
1991\end{array}$ & $\begin{array}{l}8.8 \\
3.2 \\
0.0 \\
4.5\end{array}$ & $\begin{array}{r}27 \\
10 \\
0 \\
14\end{array}$ & $\begin{array}{l}M \\
M \\
M \\
M\end{array}$ & \\
\hline \multirow[t]{4}{*}{ WW-5a } & 364635115572901 & 364635 & 1155729 & $03-23-51$ & 3,093 & 887 & i. 642 & $\begin{array}{l}877 \\
910\end{array}$ & $\stackrel{P}{X}$ & A & $\mathrm{AM}$ & $\begin{array}{l}1951 \\
1952 \\
1953 \\
1954 \\
1955\end{array}$ & $\begin{array}{l}9.7 \\
9.7 \\
9.7 \\
9.7 \\
9.7\end{array}$ & $\begin{array}{l}30 \\
30 \\
30 \\
30 \\
30\end{array}$ & $\begin{array}{l}\mathrm{Z} \\
\mathrm{Z} \\
\mathrm{Z} \\
\mathrm{Z} \\
\mathrm{Z}\end{array}$ & \\
\hline & & & & & & & & & & & & $\begin{array}{l}1956 \\
1957 \\
1958 \\
1959 \\
1960\end{array}$ & $\begin{array}{r}9.7 \\
9.7 \\
7.2 \\
9.8 \\
12.7\end{array}$ & $\begin{array}{l}30 \\
30 \\
22 \\
30 \\
39\end{array}$ & $\begin{array}{l}\mathrm{Z} \\
\mathrm{Z} \\
\mathrm{R} \\
\mathrm{R} \\
\mathrm{R}\end{array}$ & \\
\hline & & & & & & & & & & & & $\begin{array}{l}1961 \\
1962 \\
1963 \\
1964 \\
1965\end{array}$ & $\begin{array}{r}19.5 \\
34.5 \\
23.8 \\
19.5 \\
9.8\end{array}$ & $\begin{array}{r}60 \\
106 \\
73 \\
60 \\
30\end{array}$ & $\begin{array}{l}\mathrm{R} \\
\mathrm{R} \\
\mathrm{R} \\
\mathrm{R} \\
\mathrm{R}\end{array}$ & \\
\hline & & & & & & & & & & & & $\begin{array}{l}1966 \\
1967 \\
1968 \\
1969 \\
1970 \\
1971\end{array}$ & $\begin{array}{r}16.6 \\
14.7 \\
16.2 \\
5.7 \\
3.5 \\
0.0\end{array}$ & $\begin{array}{r}51 \\
45 \\
50 \\
17 \\
11 \\
0\end{array}$ & $\begin{array}{l}\mathrm{R} \\
\mathrm{R} \\
\mathrm{R} \\
\mathrm{R} \\
\mathrm{R} \\
\mathrm{R}\end{array}$ & \\
\hline \multirow[t]{2}{*}{ WW-5b } & 364805115580801 & 364805 & 1155808 & $05-07-51$ & 3,092 & 900 & 700 & 900 & $\mathbf{P}$ & A & $\mathrm{AM}$ & $\begin{array}{l}1951 \\
1952 \\
1953 \\
1954 \\
1955\end{array}$ & $\begin{array}{l}20.3 \\
20.3 \\
20.3 \\
20.3 \\
20.3\end{array}$ & $\begin{array}{l}62 \\
62 \\
62 \\
62 \\
62\end{array}$ & $\begin{array}{l}\mathrm{Z} \\
\mathrm{Z} \\
\mathrm{Z} \\
\mathrm{Z} \\
\mathrm{Z}\end{array}$ & \\
\hline & & & & & & & & & & & & $\begin{array}{l}1956 \\
1957 \\
1958 \\
1959 \\
1960\end{array}$ & $\begin{array}{l}20.3 \\
20.3 \\
26.3 \\
17.9 \\
21.7\end{array}$ & $\begin{array}{l}62 \\
62 \\
81 \\
55 \\
67\end{array}$ & $\begin{array}{l}\mathrm{Z} \\
\mathrm{Z} \\
\mathrm{R} \\
\mathrm{R} \\
\mathrm{R}\end{array}$ & \\
\hline
\end{tabular}


Table 3. Annual withdrawals from ground-water supply wells at the Nevada Test Site (NTS), Nye County, Nevada, calendar years 1951-91--Continued

\begin{tabular}{|c|c|c|c|c|c|c|c|c|c|c|c|c|c|c|c|c|}
\hline \multirow{2}{*}{$\begin{array}{l}\text { NTS hole } \\
\text { number }\end{array}$} & \multirow{2}{*}{$\begin{array}{c}\text { USGS } \\
\text { standard } \\
\text { identification }\end{array}$} & \multirow{2}{*}{$\begin{array}{l}\text { Latitude } \\
\text { (degrees, } \\
\text { minutes, } \\
\text { seconds) }\end{array}$} & \multirow{2}{*}{$\begin{array}{l}\text { Longitude } \\
\text { (degrees, } \\
\text { minutes, } \\
\text { seconds) }\end{array}$} & \multirow{2}{*}{$\begin{array}{l}\text { Date well } \\
\text { completed } \\
\text { (month, } \\
\text { day, year) }\end{array}$} & \multirow{2}{*}{$\begin{array}{l}\text { Land- } \\
\text { surface } \\
\text { altitude } \\
\text { (feet) }\end{array}$} & \multirow{2}{*}{$\begin{array}{l}\text { Well } \\
\text { depth } \\
\text { (feet) }\end{array}$} & \multicolumn{3}{|c|}{$\begin{array}{c}\text { Depth of } \\
\text { open interval }\end{array}$} & \multirow{2}{*}{$\begin{array}{c}\text { Primary } \\
\text { water- } \\
\text { yielding } \\
\text { units }\end{array}$} & \multirow{2}{*}{$\begin{array}{l}\text { Ground- } \\
\text { water } \\
\text { subbasin }\end{array}$} & \multicolumn{5}{|c|}{ Ground-water withdrawal } \\
\hline & & & & & & & $\begin{array}{c}\text { Top } \\
\text { (feet) }\end{array}$ & $\begin{array}{c}\text { Bottom } \\
\text { (feet) }\end{array}$ & Type & & & $\begin{array}{l}\text { Calendar } \\
\text { year }\end{array}$ & $\begin{array}{l}\text { Million } \\
\text { gallons }\end{array}$ & $\begin{array}{c}\text { Acre- } \\
\text { feet }\end{array}$ & Source & $\begin{array}{c}\text { Days } \\
\text { estimated }\end{array}$ \\
\hline \multirow[t]{4}{*}{ WW-5b } & 364805115580801 & 364805 & 1155808 & $05-07-51$ & 3,092 & 900 & 700 & 900 & $P$ & A & $\mathrm{AM}$ & $\begin{array}{l}1961 \\
1962 \\
1963 \\
1964 \\
1965\end{array}$ & $\begin{array}{l}37.2 \\
66.1 \\
23.3 \\
30.8 \\
23.2\end{array}$ & $\begin{array}{r}114 \\
203 \\
72 \\
95 \\
71\end{array}$ & $\begin{array}{l}\mathrm{R} \\
\mathrm{R} \\
\mathrm{R} \\
\mathrm{R} \\
\mathrm{R}\end{array}$ & \\
\hline & & & & & & & & & & & & $\begin{array}{l}1966 \\
1967 \\
1968 \\
1969 \\
1970\end{array}$ & $\begin{array}{r}35.9 \\
9.0 \\
10.4 \\
6.8 \\
23.6\end{array}$ & $\begin{array}{r}110 \\
28 \\
32 \\
21 \\
72\end{array}$ & $\begin{array}{l}R \\
R \\
R \\
R \\
R\end{array}$ & \\
\hline & & & & & & & & & & & & $\begin{array}{l}1971 \\
1983 \\
1984 \\
1985 \\
1986\end{array}$ & $\begin{array}{r}8.1 \\
32.2 \\
57.9 \\
67.8 \\
58.5\end{array}$ & $\begin{array}{r}25 \\
99 \\
178 \\
208 \\
180\end{array}$ & $\begin{array}{l}\mathrm{R} \\
\mathrm{M} \\
\mathrm{E} \\
\mathrm{E} \\
\mathrm{M}\end{array}$ & $\begin{array}{r}8 \\
20\end{array}$ \\
\hline & & & & & & & & & & & & $\begin{array}{l}1987 \\
1988 \\
1989 \\
1990 \\
1991\end{array}$ & $\begin{array}{r}50.5 \\
57.5 \\
0.0 \\
0.0 \\
0.0\end{array}$ & $\begin{array}{r}155 \\
176 \\
0 \\
0 \\
0\end{array}$ & $\begin{array}{l}\mathrm{M} \\
\mathrm{M} \\
\mathrm{M} \\
\mathrm{M} \\
\mathrm{M}\end{array}$ & \\
\hline \multirow[t]{4}{*}{ WW-5c } & 364708115574401 & 364720 & 1155749 & $03-24-54$ & 3,081 & 1,200 & $\begin{array}{r}887 \\
1,187\end{array}$ & $\begin{array}{l}1,187 \\
1,200\end{array}$ & $\begin{array}{l}\mathrm{P} \\
\mathrm{X}\end{array}$ & A & $\mathrm{AM}$ & $\begin{array}{l}1954 \\
1955 \\
1956 \\
1957 \\
1958\end{array}$ & $\begin{array}{l}18.1 \\
18.1 \\
18.1 \\
18.1 \\
20.2\end{array}$ & $\begin{array}{l}56 \\
56 \\
56 \\
56 \\
62\end{array}$ & $\begin{array}{l}\mathbf{Z} \\
\mathbf{Z} \\
\mathbf{Z} \\
\mathbf{Z} \\
\mathrm{R}\end{array}$ & \\
\hline & & & & & & & & & & & & $\begin{array}{l}1959 \\
1960 \\
1961 \\
1962 \\
1963\end{array}$ & $\begin{array}{l}12.8 \\
19.6 \\
22.1 \\
72.9 \\
69.5\end{array}$ & $\begin{array}{r}39 \\
60 \\
68 \\
224 \\
213\end{array}$ & $\begin{array}{l}\mathrm{R} \\
\mathrm{R} \\
\mathrm{R} \\
\mathrm{R} \\
\mathrm{R}\end{array}$ & \\
\hline & & & & & & & & & & & & $\begin{array}{l}1964 \\
1965 \\
1966 \\
1967 \\
1968\end{array}$ & $\begin{array}{l}48.3 \\
58.0 \\
56.6 \\
43.6 \\
59.5\end{array}$ & $\begin{array}{l}148 \\
178 \\
174 \\
134 \\
183\end{array}$ & $\begin{array}{l}\mathrm{R} \\
\mathrm{R} \\
\mathrm{R} \\
\mathrm{R} \\
\mathrm{R}\end{array}$ & \\
\hline & & & & & & & & & & & & $\begin{array}{l}1969 \\
1970 \\
1971 \\
1983 \\
1984\end{array}$ & $\begin{array}{l}39.9 \\
41.1 \\
44.2 \\
42.1 \\
58.7\end{array}$ & $\begin{array}{l}122 \\
126 \\
136 \\
129 \\
180\end{array}$ & $\begin{array}{l}\mathrm{R} \\
\mathrm{R} \\
\mathrm{R} \\
\mathrm{M} \\
\mathrm{E}\end{array}$ & 8 \\
\hline
\end{tabular}


N Table 3. Annual withdrawals from ground-water supply wells at the Nevada Test Site (NTS), Nye County, Nevada, calendar years 1951-91--Continued

\begin{tabular}{|c|c|c|c|c|c|c|c|c|c|c|c|c|c|c|c|c|}
\hline \multirow{2}{*}{$\begin{array}{l}\text { NTS hole } \\
\text { number }\end{array}$} & \multirow{2}{*}{$\begin{array}{c}\text { USGS } \\
\text { standard } \\
\text { identification }\end{array}$} & \multirow{2}{*}{$\begin{array}{l}\text { Latitude } \\
\text { (degrees, } \\
\text { minutes, } \\
\text { seconds) }\end{array}$} & \multirow{2}{*}{$\begin{array}{l}\text { Longitude } \\
\text { (degrees, } \\
\text { minutes, } \\
\text { seconds) }\end{array}$} & \multirow{2}{*}{$\begin{array}{l}\text { Date well } \\
\text { completed } \\
\text { (month, } \\
\text { day, year) }\end{array}$} & \multirow{2}{*}{$\begin{array}{l}\text { Land- } \\
\text { surface } \\
\text { altitude } \\
\text { (feet) }\end{array}$} & \multirow{2}{*}{$\begin{array}{l}\text { Well } \\
\text { depth } \\
\text { (feet) }\end{array}$} & \multicolumn{3}{|c|}{$\begin{array}{c}\text { Depth of } \\
\text { open interval }\end{array}$} & \multirow{2}{*}{$\begin{array}{c}\text { Primary } \\
\text { water- } \\
\text { yielding } \\
\text { units }\end{array}$} & \multirow{2}{*}{$\begin{array}{l}\text { Ground- } \\
\text { water } \\
\text { subbasin }\end{array}$} & \multicolumn{5}{|c|}{ Ground-water withdrawal } \\
\hline & & & & & & & $\begin{array}{c}\text { Top } \\
\text { (feet) }\end{array}$ & $\begin{array}{c}\text { Bottom } \\
\text { (feet) }\end{array}$ & Type & & & $\begin{array}{l}\text { Calendar } \\
\text { year }\end{array}$ & $\begin{array}{l}\text { Million } \\
\text { gallons }\end{array}$ & $\begin{array}{c}\text { Acre- } \\
\text { feet }\end{array}$ & Source & $\begin{array}{c}\text { Days } \\
\text { estimated }\end{array}$ \\
\hline \multirow[t]{2}{*}{ WW-5c } & 364708115574401 & 364720 & 1155749 & $03-24-54$ & 3,081 & 1,200 & $\begin{array}{r}887 \\
1,187\end{array}$ & $\begin{array}{l}1,187 \\
1,200\end{array}$ & $\begin{array}{l}\mathrm{P} \\
\mathrm{X}\end{array}$ & A & AM & $\begin{array}{l}1985 \\
1986 \\
1987 \\
1988 \\
1989\end{array}$ & $\begin{array}{l}47.1 \\
64.0 \\
50.3 \\
67.8 \\
35.4\end{array}$ & $\begin{array}{l}145 \\
196 \\
154 \\
208 \\
109\end{array}$ & $\begin{array}{l}\mathrm{E} \\
\mathrm{M} \\
\mathrm{M} \\
\mathrm{M} \\
\mathrm{M}\end{array}$ & 20 \\
\hline & & & & & & & & & & & & $\begin{array}{l}1990 \\
1991\end{array}$ & $\begin{array}{l}38.4 \\
28.8\end{array}$ & $\begin{array}{r}118 \\
88\end{array}$ & $\begin{array}{l}\mathrm{M} \\
\mathrm{M}\end{array}$ & \\
\hline \multicolumn{17}{|c|}{ AREA 6} \\
\hline $\begin{array}{l}W W-3^{3} \\
W W-3^{3}\end{array}$ & 365942116032900 & 365943 & 1160329 & $02-10-51$ & 3,969 & 1,575 & 1,209 & 1,575 & $\mathrm{x}$ & $A, V$ & $\mathrm{AM}$ & 1951 & 2.8 & 9 & $\mathrm{z}$ & \\
\hline \multirow{4}{*}{ WW- $3^{3}$} & 365942116032901 & 365943 & 1160329 & $03-05-52$ & 3,969 & 1,800 & $\begin{array}{l}1,535 \\
1,765\end{array}$ & $\begin{array}{l}1,765 \\
1,800\end{array}$ & $\begin{array}{l}\mathrm{P} \\
\mathrm{X}\end{array}$ & $A, V$ & $\mathrm{AM}$ & $\begin{array}{l}1952 \\
1953 \\
1954 \\
1955 \\
1956\end{array}$ & $\begin{array}{l}2.8 \\
2.8 \\
2.8 \\
2.8 \\
2.8\end{array}$ & $\begin{array}{l}9 \\
9 \\
9 \\
9 \\
9\end{array}$ & $\begin{array}{l}\mathrm{Z} \\
\mathbf{Z} \\
\mathbf{Z} \\
\mathrm{Z} \\
\mathrm{Z}\end{array}$ & \\
\hline & & & & & & & & & & & & $\begin{array}{l}1957 \\
1958 \\
1959 \\
1960 \\
1961\end{array}$ & $\begin{array}{r}2.8 \\
12.6 \\
17.2 \\
11.2 \\
13.0\end{array}$ & $\begin{array}{r}9 \\
39 \\
53 \\
34 \\
40\end{array}$ & $\begin{array}{l}Z \\
R \\
R \\
R \\
R\end{array}$ & \\
\hline & & & & & & & & & & & & $\begin{array}{l}1962 \\
1963 \\
1964 \\
1965 \\
1966\end{array}$ & $\begin{array}{l}22.0 \\
21.8 \\
20.2 \\
16.1 \\
18.2\end{array}$ & $\begin{array}{l}68 \\
67 \\
62 \\
49 \\
56\end{array}$ & $\begin{array}{l}\mathrm{R} \\
\mathrm{R} \\
\mathrm{R} \\
\mathrm{R} \\
\mathrm{R}\end{array}$ & \\
\hline & & & & & & & & & & & & $\begin{array}{l}1967 \\
1968 \\
1969 \\
1970 \\
1971\end{array}$ & $\begin{array}{r}14.9 \\
7.4 \\
6.8 \\
1.8 \\
0.0\end{array}$ & $\begin{array}{r}46 \\
23 \\
21 \\
6 \\
0\end{array}$ & $\begin{array}{l}\mathrm{R} \\
\mathrm{R} \\
\mathrm{R} \\
\mathrm{R} \\
\mathrm{R}\end{array}$ & \\
\hline \multirow[t]{2}{*}{ Ww-4 } & 365418116012601 & 365418 & 1160126 & $11-18-81$ & 3,602 & 1,479 & $\begin{array}{r}942 \\
1,438\end{array}$ & $\begin{array}{l}1,436 \\
1,479\end{array}$ & $\stackrel{P}{\mathrm{X}}$ & $\mathrm{v}$ & $\mathrm{AM}$ & $\begin{array}{l}1983 \\
1984 \\
1985 \\
1986 \\
1987\end{array}$ & $\begin{array}{l}24.1 \\
48.4 \\
42.9 \\
28.2 \\
28.2\end{array}$ & $\begin{array}{r}74 \\
149 \\
132 \\
86 \\
87\end{array}$ & $\begin{array}{l}\mathrm{M} \\
\mathrm{E} \\
\mathrm{E} \\
\mathrm{M} \\
\mathrm{M}\end{array}$ & $\begin{array}{r}8 \\
20\end{array}$ \\
\hline & & & & & & & & & & & & $\begin{array}{l}1988 \\
1989 \\
1990 \\
1991\end{array}$ & $\begin{array}{r}46.7 \\
192.0 \\
68.2 \\
70.7\end{array}$ & $\begin{array}{l}143 \\
589 \\
209 \\
217\end{array}$ & $\begin{array}{l}\mathrm{M} \\
\mathrm{M} \\
\mathrm{E} \\
\mathrm{M}\end{array}$ & 28 \\
\hline$W W-4 a^{4}$ & 365412116013901 & 365412 & 1160139 & $02-21-90$ & 3,606 & 1,502 & $\begin{array}{l}1,066 \\
1,365 \\
1,501\end{array}$ & $\begin{array}{l}1,281 \\
1,457 \\
1,516\end{array}$ & $\begin{array}{l}\mathrm{P} \\
\mathrm{P} \\
\mathrm{X}\end{array}$ & $\mathrm{V}$ & $\mathrm{AM}$ & -- & - & -- & - & \\
\hline
\end{tabular}


Table 3. Annual withdrawals from ground-water supply wells at the Nevada Test Site (NTS), Nye County, Nevada, calendar years 1951-91--Continued

\begin{tabular}{|c|c|c|c|c|c|c|c|c|c|c|c|c|c|c|c|c|}
\hline \multirow{2}{*}{$\begin{array}{l}\text { NTS hole } \\
\text { number }\end{array}$} & \multirow{2}{*}{$\begin{array}{c}\text { USGS } \\
\text { standard } \\
\text { identification }\end{array}$} & \multirow{2}{*}{$\begin{array}{c}\text { Latitude } \\
\text { (degrees, } \\
\text { minutes, } \\
\text { seconds) }\end{array}$} & \multirow{2}{*}{$\begin{array}{l}\text { Longitude } \\
\text { (degrees, } \\
\text { minutes, } \\
\text { seconds) }\end{array}$} & \multirow{2}{*}{$\begin{array}{l}\text { Date well } \\
\text { completed } \\
\text { (month, } \\
\text { day, year) }\end{array}$} & \multirow{2}{*}{$\begin{array}{l}\text { Land- } \\
\text { surface } \\
\text { altitude } \\
\text { (feet) }\end{array}$} & \multirow{2}{*}{$\begin{array}{l}\text { Well } \\
\text { depth } \\
\text { (feet) }\end{array}$} & \multicolumn{3}{|c|}{$\begin{array}{c}\text { Depth of } \\
\text { open interval }\end{array}$} & \multirow{2}{*}{$\begin{array}{l}\text { Primary } \\
\text { water- } \\
\text { yielding } \\
\text { units }\end{array}$} & \multirow{2}{*}{$\begin{array}{l}\text { Ground- } \\
\text { water } \\
\text { subbasin }\end{array}$} & \multicolumn{5}{|c|}{ Ground-water withdrawal } \\
\hline & & & & & & & $\begin{array}{c}\text { Top } \\
\text { (feet) }\end{array}$ & $\begin{array}{c}\text { Bottom } \\
\text { (feet) }\end{array}$ & Type & & & $\begin{array}{c}\text { Calendar } \\
\text { year }\end{array}$ & $\begin{array}{l}\text { Million } \\
\text { gallons }\end{array}$ & $\begin{array}{c}\text { Acre- } \\
\text { feet }\end{array}$ & Source & $\begin{array}{c}\text { Days } \\
\text { estimated }\end{array}$ \\
\hline$\overline{W W-C^{3}}$ & 365508116003501 & 365508 & 1160035 & $03-30-61$ & 3,921 & 1,650 & 1,571 & 1,679 & $P$ & $\mathrm{C}$ & $\mathrm{AM}$ & $\begin{array}{l}1961 \\
1962 \\
1963 \\
1964 \\
1965 \\
1966\end{array}$ & $\begin{array}{r}4.9 \\
37.4 \\
43.0 \\
56.9 \\
30.9 \\
34.0\end{array}$ & $\begin{array}{r}15 \\
115 \\
132 \\
175 \\
95 \\
104\end{array}$ & $\begin{array}{l}\mathbf{R} \\
\mathbf{R} \\
\mathbf{R} \\
\mathbf{R} \\
\mathbf{R} \\
\mathbf{R}\end{array}$ & \\
\hline \multirow[t]{3}{*}{$W W-C^{3}$} & 365508116003502 & 365508 & 1160035 & $04-30-67$ & 3,921 & 1,624 & $\begin{array}{l}1,560 \\
1,624\end{array}$ & $\begin{array}{l}1,624 \\
1,701\end{array}$ & $\stackrel{P}{X}$ & C & $\mathrm{AM}$ & $\begin{array}{l}1967 \\
1968 \\
1969 \\
1970 \\
1971\end{array}$ & $\begin{array}{l}28.9 \\
81.0 \\
95.6 \\
62.4 \\
83.4\end{array}$ & $\begin{array}{r}89 \\
249 \\
293 \\
191 \\
256\end{array}$ & $\begin{array}{l}R \\
R \\
R \\
R \\
R \\
R\end{array}$ & \\
\hline & & & & & & & & & & & & $\begin{array}{l}1983 \\
1984 \\
1985 \\
1986 \\
1987\end{array}$ & $\begin{array}{r}27.7 \\
31.2 \\
19.4 \\
17.3 \\
7.2\end{array}$ & $\begin{array}{l}85 \\
96 \\
59 \\
53 \\
22\end{array}$ & $\begin{array}{l}M \\
E \\
E \\
E \\
M\end{array}$ & $\begin{array}{r}8 \\
20 \\
7\end{array}$ \\
\hline & & & & & & & & & & & & $\begin{array}{l}1988 \\
1989 \\
1990 \\
1991\end{array}$ & $\begin{array}{l}17.4 \\
31.6 \\
29.7 \\
27.7\end{array}$ & $\begin{array}{l}53 \\
97 \\
91 \\
85\end{array}$ & $\begin{array}{c}M \\
M \\
E \\
M\end{array}$ & 7 \\
\hline \multirow[t]{4}{*}{ Ww-C-1 } & 365500116003901 & 365500 & 1160039 & $06-09-62$ & 3,921 & 1,707 & $\begin{array}{l}1,536 \\
1,650\end{array}$ & $\begin{array}{l}1,650 \\
1,707\end{array}$ & $\begin{array}{l}\mathrm{P} \\
\mathrm{X}\end{array}$ & C & $\mathrm{AM}$ & $\begin{array}{l}1962 \\
1963 \\
1964 \\
1965 \\
1966\end{array}$ & $\begin{array}{l}16.2 \\
31.5 \\
70.6 \\
39.1 \\
76.0\end{array}$ & $\begin{array}{r}50 \\
97 \\
217 \\
120 \\
233\end{array}$ & $\begin{array}{l}\mathrm{R} \\
\mathrm{R} \\
\mathrm{R} \\
\mathrm{R} \\
\mathrm{R}\end{array}$ & \\
\hline & & & & & & & & & & & & $\begin{array}{l}1967 \\
1968 \\
1969 \\
1970 \\
1971\end{array}$ & $\begin{array}{l}34.0 \\
26.9 \\
36.4 \\
18.3 \\
20.4\end{array}$ & $\begin{array}{r}104 \\
83 \\
112 \\
56 \\
63\end{array}$ & $\begin{array}{l}\mathrm{R} \\
\mathrm{R} \\
\mathrm{R} \\
\mathrm{R} \\
\mathrm{R}\end{array}$ & \\
\hline & & & & & & & & & & & & $\begin{array}{l}1983 \\
1984 \\
1985 \\
1986 \\
1987\end{array}$ & $\begin{array}{l}37.6 \\
23.3 \\
29.0 \\
30.1 \\
22.3\end{array}$ & $\begin{array}{r}115 \\
72 \\
89 \\
92 \\
69\end{array}$ & $\begin{array}{l}\mathrm{M} \\
\mathrm{E} \\
\mathrm{E} \\
\mathrm{M} \\
\mathrm{M}\end{array}$ & $\begin{array}{r}8 \\
20\end{array}$ \\
\hline & & & & & & & & & & & & $\begin{array}{l}1988 \\
1989 \\
1990 \\
1991\end{array}$ & $\begin{array}{l}24.8 \\
28.1 \\
23.3 \\
23.8\end{array}$ & $\begin{array}{l}76 \\
86 \\
72 \\
73\end{array}$ & $\begin{array}{l}\mathrm{E} \\
\mathrm{M} \\
\mathrm{M} \\
\mathrm{M}\end{array}$ & 7 \\
\hline
\end{tabular}


I Table 3. Annual withdrawals from ground-water supply wells at the Nevada Test Site (NTS), Nye County, Nevada, calendar years 1951-91--Continued

\begin{tabular}{|c|c|c|c|c|c|c|c|c|c|c|c|c|c|c|c|c|}
\hline \multirow{2}{*}{$\begin{array}{l}\text { NTS hole } \\
\text { number }\end{array}$} & \multirow{2}{*}{$\begin{array}{c}\text { USGS } \\
\text { standard } \\
\text { Identiflcation }\end{array}$} & \multirow{2}{*}{$\begin{array}{c}\text { Latitude } \\
\text { (degrees, } \\
\text { minutes, } \\
\text { seconds) }\end{array}$} & \multirow{2}{*}{$\begin{array}{l}\text { Longitude } \\
\text { (degrees, } \\
\text { minutes, } \\
\text { seconds) }\end{array}$} & \multirow{2}{*}{$\begin{array}{l}\text { Date well } \\
\text { completed } \\
\text { (month, } \\
\text { day, year) }\end{array}$} & \multirow{2}{*}{$\begin{array}{l}\text { Land- } \\
\text { surface } \\
\text { altitude } \\
\text { (feet) }\end{array}$} & \multirow{2}{*}{$\begin{array}{l}\text { Well } \\
\text { depth } \\
\text { (feet) }\end{array}$} & \multicolumn{3}{|c|}{$\begin{array}{l}\text { Depth of } \\
\text { open interval }\end{array}$} & \multirow{2}{*}{$\begin{array}{c}\text { Primany } \\
\text { water- } \\
\text { yielding } \\
\text { units }\end{array}$} & \multirow{2}{*}{$\begin{array}{l}\text { Ground- } \\
\text { water } \\
\text { subbasin }\end{array}$} & \multicolumn{5}{|c|}{ Ground-water withdrawal } \\
\hline & & & & & & & $\begin{array}{c}\text { Top } \\
\text { (feet) }\end{array}$ & $\begin{array}{c}\text { Bottom } \\
\text { (feet) }\end{array}$ & Type & & & $\begin{array}{c}\text { Calendar } \\
\text { year }\end{array}$ & $\begin{array}{l}\text { Millon } \\
\text { gallons }\end{array}$ & $\begin{array}{c}\text { Acre- } \\
\text { feet }\end{array}$ & Source & $\begin{array}{c}\text { Days } \\
\text { estimated }\end{array}$ \\
\hline \multicolumn{17}{|c|}{ AREA 15} \\
\hline UE-15d Ww ${ }^{1.5}$ & 371230116021501 & 371233 & 1160229 & $04-17-62$ & 4,586 & 6,001 & 5,400 & 6,001 & $\mathrm{x}$ & C & $\mathrm{AM}$ & $\begin{array}{l}1966 \\
1967 \\
1968 \\
1969 \\
1970 \\
1971\end{array}$ & $\begin{array}{l}17.1 \\
37.3 \\
40.3 \\
37.7 \\
37.4 \\
27.0\end{array}$ & $\begin{array}{r}52 \\
114 \\
124 \\
116 \\
115 \\
83\end{array}$ & $\begin{array}{l}\mathrm{R} \\
\mathrm{R} \\
\mathrm{R} \\
\mathrm{R} \\
\mathrm{R} \\
\mathrm{R}\end{array}$ & \\
\hline \multicolumn{17}{|c|}{ AREA 16} \\
\hline UE-16d WW & 370412116095101 & 370412 & 1160951 & $03-03-81$ & 4,684 & 3,000 & 1,145 & 1,310 & $P$ & $\mathrm{C}$ & $\mathrm{AM}$ & $\begin{array}{l}1983 \\
1984 \\
1985 \\
1986 \\
1987\end{array}$ & $\begin{array}{r}4.6 \\
8.1 \\
25.4 \\
38.5 \\
34.0\end{array}$ & $\begin{array}{r}14 \\
25 \\
78 \\
118 \\
104\end{array}$ & $\begin{array}{l}\mathrm{M} \\
\mathrm{E} \\
\mathrm{E} \\
\mathrm{M} \\
\mathrm{M}\end{array}$ & $\begin{array}{r}8 \\
20\end{array}$ \\
\hline & & & & & & & & & & & & $\begin{array}{l}1988 \\
1989 \\
1990 \\
1991\end{array}$ & $\begin{array}{l}38.1 \\
26.0 \\
32.9 \\
27.3\end{array}$ & $\begin{array}{r}117 \\
80 \\
101 \\
84\end{array}$ & $\begin{array}{l}M \\
M \\
M \\
M\end{array}$ & \\
\hline \multicolumn{17}{|c|}{ AREA 18} \\
\hline WW-8 & 370956116172101 & 370956 & 1161721 & $01-07-63$ & 5,695 & 1,862 & $\begin{array}{l}1,250 \\
1,450 \\
1,630\end{array}$ & $\begin{array}{l}1,300 \\
1,500 \\
1,780\end{array}$ & $\begin{array}{l}\mathrm{P} \\
\mathrm{P} \\
\mathrm{P}\end{array}$ & $\mathrm{v}$ & AFFCR & $\begin{array}{l}1963 \\
1964 \\
1965 \\
1966 \\
1967\end{array}$ & $\begin{array}{r}5.3 \\
111.9 \\
32.3 \\
36.2 \\
57.5\end{array}$ & $\begin{array}{r}16 \\
343 \\
99 \\
111 \\
176\end{array}$ & $\begin{array}{l}\mathrm{R} \\
\mathrm{R} \\
\mathrm{R} \\
\mathrm{R} \\
\mathrm{R}\end{array}$ & \\
\hline & & & & & & & & & & & & $\begin{array}{l}1983 \\
1984 \\
1985 \\
1986 \\
1987\end{array}$ & $\begin{array}{l}58.9 \\
61.1 \\
60.2 \\
37.9 \\
68.4\end{array}$ & $\begin{array}{l}181 \\
188 \\
185 \\
116 \\
210\end{array}$ & $\begin{array}{l}M \\
E \\
E \\
M \\
M\end{array}$ & $\begin{array}{r}8 \\
20\end{array}$ \\
\hline & & & & & & & & & & & & $\begin{array}{l}1988 \\
1989 \\
1990 \\
1991\end{array}$ & $\begin{array}{l}65.3 \\
53.9 \\
42.9 \\
57.6\end{array}$ & $\begin{array}{l}200 \\
165 \\
132 \\
177\end{array}$ & $\begin{array}{l}M \\
E \\
M \\
M\end{array}$ & 28 \\
\hline \multicolumn{17}{|c|}{ AREA 19} \\
\hline UE-19c WW & 371608116191002 & 371608 & 1161910 & $06-30-75$ & 7,033 & 8,489 & $\begin{array}{l}2,421 \\
3,209\end{array}$ & $\begin{array}{l}3,209 \\
8,489\end{array}$ & $\begin{array}{l}\mathrm{X} \\
\mathrm{X}\end{array}$ & $\mathrm{v}$ & AFFCR & $\begin{array}{l}1983 \\
1984 \\
1985 \\
1986 \\
1987\end{array}$ & $\begin{array}{r}41.8 \\
64.1 \\
103.2 \\
85.1 \\
51.1\end{array}$ & $\begin{array}{l}128 \\
197 \\
317 \\
261 \\
157\end{array}$ & $\begin{array}{l}\mathrm{M} \\
\mathrm{E} \\
\mathrm{E} \\
\mathrm{M} \\
\mathrm{M}\end{array}$ & $\begin{array}{r}8 \\
20\end{array}$ \\
\hline
\end{tabular}


Table 3. Annual withdrawals from ground-water supply wells at the Nevada Test Site (NTS), Nye County, Nevada, calendar years 1951-91--Continued

\begin{tabular}{|c|c|c|c|c|c|c|c|c|c|c|c|c|c|c|c|c|}
\hline \multirow{2}{*}{$\begin{array}{l}\text { NTS hole } \\
\text { number }\end{array}$} & \multirow{2}{*}{$\begin{array}{c}\text { USGS } \\
\text { standard } \\
\text { identification }\end{array}$} & \multirow{2}{*}{$\begin{array}{l}\text { Latitude } \\
\text { (degrees, } \\
\text { minutes, } \\
\text { seconds) }\end{array}$} & \multirow{2}{*}{$\begin{array}{l}\text { Longitude } \\
\text { (degrees, } \\
\text { minutes, } \\
\text { seconds) }\end{array}$} & \multirow{2}{*}{$\begin{array}{l}\text { Date well } \\
\text { completed } \\
\text { (month, } \\
\text { day, year) }\end{array}$} & \multirow{2}{*}{$\begin{array}{l}\text { Land- } \\
\text { surface } \\
\text { altitude } \\
\text { (feet) }\end{array}$} & \multirow{2}{*}{$\begin{array}{l}\text { Well } \\
\text { depth } \\
\text { (feet) }\end{array}$} & \multicolumn{3}{|c|}{$\begin{array}{l}\text { Depth of } \\
\text { open interval }\end{array}$} & \multirow{2}{*}{$\begin{array}{l}\text { Primary } \\
\text { water- } \\
\text { yielding } \\
\text { units }\end{array}$} & \multirow{2}{*}{$\begin{array}{c}\text { Ground- } \\
\text { water } \\
\text { subbasin }\end{array}$} & \multicolumn{5}{|c|}{ Ground-water withdrawal } \\
\hline & & & & & & & $\begin{array}{c}\text { Top } \\
\text { (feet) }\end{array}$ & $\begin{array}{c}\text { Bottom } \\
\text { (feet) }\end{array}$ & Type & & & $\begin{array}{l}\text { Calendar } \\
\text { year }\end{array}$ & $\begin{array}{l}\text { Million } \\
\text { gallons }\end{array}$ & $\begin{array}{c}\text { Acre- } \\
\text { feet }\end{array}$ & Source & $\begin{array}{c}\text { Days } \\
\text { estimated }\end{array}$ \\
\hline UE-19c WW & 371608116191002 & 371608 & 1161910 & $06-30-75$ & 7,033 & 8,489 & $\begin{array}{l}2,421 \\
3,209\end{array}$ & $\begin{array}{l}3,209 \\
8,489\end{array}$ & $\begin{array}{l}\mathrm{X} \\
\mathrm{X}\end{array}$ & $\mathrm{v}$ & AFFCR & $\begin{array}{l}1988 \\
1989 \\
1990 \\
1991\end{array}$ & $\begin{array}{r}64.3 \\
138.6 \\
110.6 \\
126.1\end{array}$ & $\begin{array}{l}197 \\
425 \\
339 \\
387\end{array}$ & $\begin{array}{l}\mathrm{M} \\
\mathrm{M} \\
\mathrm{M} \\
\mathrm{M}\end{array}$ & \\
\hline UE-19e WW & 371750116195901 & 371750 & 1161959 & $08-22-64$ & 6,919 & 6,005 & 2,475 & 6,005 & $\mathrm{x}$ & $\mathrm{v}$ & AFFCR & $\begin{array}{l}1965 \\
1966 \\
1967\end{array}$ & $\begin{array}{r}11.2 \\
6.3 \\
10.2\end{array}$ & $\begin{array}{l}34 \\
19 \\
31\end{array}$ & $\begin{array}{l}\mathrm{R} \\
\mathrm{R} \\
\mathrm{R}\end{array}$ & \\
\hline UE-19gS WW6 & 371830116215303 & 371830 & 1162153 & $05-09-65$ & 6,719 & 7,506 & $\begin{array}{l}2,650 \\
4,350 \\
4,508\end{array}$ & $\begin{array}{l}4,113 \\
4,508 \\
7,500\end{array}$ & $\underset{\mathrm{X}}{\mathrm{X}}$ & $\mathrm{v}$ & AFFCR & 1967 & 21.0 & 64 & $\mathrm{R}$ & \\
\hline \multicolumn{17}{|c|}{ AREA 20} \\
\hline \multirow[t]{2}{*}{ U-20 Ww } & 371505116254501 & 371505 & 1162545 & $07-22-85$ & 6,468 & 3,268 & $\begin{array}{l}2,271 \\
3,199\end{array}$ & $\begin{array}{l}3,035 \\
3,268\end{array}$ & $\begin{array}{l}\mathrm{P} \\
\mathrm{X}\end{array}$ & $\mathrm{v}$ & AFFCR & $\begin{array}{l}1985 \\
1986 \\
1987 \\
1988 \\
1989\end{array}$ & $\begin{array}{r}41.6 \\
76.2 \\
68.7 \\
105.4 \\
112.6\end{array}$ & $\begin{array}{l}128 \\
234 \\
211 \\
324 \\
345\end{array}$ & $\begin{array}{l}E \\
E \\
M \\
M \\
M\end{array}$ & $\begin{array}{l}7 \\
7\end{array}$ \\
\hline & & & & & & & & & & & & $\begin{array}{l}1990 \\
1991\end{array}$ & $\begin{array}{l}92.9 \\
30.8\end{array}$ & $\begin{array}{r}285 \\
94\end{array}$ & $\begin{array}{l}M \\
M\end{array}$ & \\
\hline $\mathrm{U}-20 \mathrm{a} 2 \mathrm{WW}^{3}$ & 371434116251602 & 371434 & 1162516 & $06-30-64$ & 6,472 & 4,500 & 2,353 & 4,500 & $\mathrm{x}$ & $\mathrm{v}$ & $\mathrm{AFFCR}$ & $\begin{array}{l}1964 \\
1965\end{array}$ & $\begin{array}{r}17.0 \\
9.1\end{array}$ & $\begin{array}{l}52 \\
28\end{array}$ & $\begin{array}{l}\mathrm{R} \\
\mathrm{R}\end{array}$ & \\
\hline U-20a $2 w_{W^{3}}$ & 371434116251603 & 371434 & 1162516 & $04-14-66$ & 6,472 & 4,500 & 2,356 & 4,500 & $\mathrm{x}$ & $\mathrm{v}$ & AFFCR & $\begin{array}{l}1966 \\
1967\end{array}$ & $\begin{array}{r}6.3 \\
22.8\end{array}$ & $\begin{array}{l}19 \\
70\end{array}$ & $\begin{array}{l}\mathrm{R} \\
\mathrm{R}\end{array}$ & \\
\hline \multicolumn{17}{|c|}{ AREA 22} \\
\hline Army $1 \mathrm{WW}$ & 363530116021401 & 363530 & 1160214 & $07-15-62$ & 3,154 & 1,953 & $\begin{array}{r}800 \\
1,368 \\
1,370 \\
1,684\end{array}$ & $\begin{array}{l}1,050 \\
1,370 \\
1,684 \\
1,953\end{array}$ & $\begin{array}{l}P \\
X \\
X \\
X\end{array}$ & C & $\mathrm{AM}$ & $\begin{array}{l}1962 \\
1963 \\
1964 \\
1965 \\
1966\end{array}$ & $\begin{array}{r}4.2 \\
2.7 \\
30.7 \\
25.4 \\
47.3\end{array}$ & $\begin{array}{r}13 \\
8 \\
94 \\
78 \\
145\end{array}$ & $\begin{array}{l}\mathrm{R} \\
\mathrm{R} \\
\mathrm{R} \\
\mathrm{R} \\
\mathrm{R}\end{array}$ & \\
\hline & & & & & & & & & & & & $\begin{array}{l}1967 \\
1968 \\
1969 \\
1970 \\
1971\end{array}$ & $\begin{array}{l}56.1 \\
52.7 \\
78.1 \\
69.4 \\
96.1\end{array}$ & $\begin{array}{l}172 \\
162 \\
240 \\
213 \\
295\end{array}$ & $\begin{array}{l}R \\
R \\
R \\
R \\
R\end{array}$ & \\
\hline & & & & & & & & & & & & $\begin{array}{l}1983 \\
1984 \\
1985 \\
1986 \\
1987\end{array}$ & $\begin{array}{l}56.8 \\
82.1 \\
41.6 \\
34.9 \\
34.7\end{array}$ & $\begin{array}{l}174 \\
252 \\
128 \\
107 \\
107\end{array}$ & $\begin{array}{l}M \\
E \\
E \\
M \\
E\end{array}$ & $\begin{array}{r}8 \\
20 \\
7\end{array}$ \\
\hline
\end{tabular}


Table 3. Annual withdrawals from ground-water supply wells at the Nevada Test Site (NTS), Nye County, Nevada, calendar years 1951-91--Continued

\begin{tabular}{|c|c|c|c|c|c|c|c|c|c|c|c|c|c|c|c|c|}
\hline \multirow{2}{*}{$\begin{array}{l}\text { NTS hole } \\
\text { number }\end{array}$} & \multirow{2}{*}{$\begin{array}{c}\text { USGS } \\
\text { standard } \\
\text { Identification }\end{array}$} & \multirow{2}{*}{$\begin{array}{l}\text { Latitude } \\
\text { (degrees, } \\
\text { minutes, } \\
\text { seconds) }\end{array}$} & \multirow{2}{*}{$\begin{array}{l}\text { Longitude } \\
\text { (degrees, } \\
\text { minutes, } \\
\text { seconds) }\end{array}$} & \multirow{2}{*}{$\begin{array}{l}\text { Date well } \\
\text { completed } \\
\text { (month, } \\
\text { day, year) }\end{array}$} & \multirow{2}{*}{$\begin{array}{l}\text { Land- } \\
\text { surface } \\
\text { altitude } \\
\text { (feet) }\end{array}$} & \multirow{2}{*}{$\begin{array}{l}\text { Well } \\
\text { depth } \\
\text { (feet) }\end{array}$} & \multicolumn{3}{|c|}{$\begin{array}{c}\text { Depth of } \\
\text { open interval }\end{array}$} & \multirow{2}{*}{$\begin{array}{l}\text { Primary } \\
\text { water- } \\
\text { yielding } \\
\text { units }\end{array}$} & \multirow{2}{*}{$\begin{array}{l}\text { Ground- } \\
\text { water } \\
\text { subbasin }\end{array}$} & \multicolumn{5}{|c|}{ Ground-water withdrawal } \\
\hline & & & & & & & $\begin{array}{c}\text { Top } \\
\text { (feet) }\end{array}$ & $\begin{array}{c}\text { Bottom } \\
\text { (feet) }\end{array}$ & Type & & & $\begin{array}{c}\text { Calendar } \\
\text { year }\end{array}$ & $\begin{array}{l}\text { Million } \\
\text { gallons }\end{array}$ & $\begin{array}{l}\text { Acre- } \\
\text { feet }\end{array}$ & Source & $\begin{array}{c}\text { Days } \\
\text { estimated }\end{array}$ \\
\hline Army $1 \mathrm{WW}$ & 363530116021401 & 363530 & 1160214 & $07-15-62$ & 3,154 & 1,953 & $\begin{array}{r}800 \\
1,368 \\
1,370 \\
1,684\end{array}$ & $\begin{array}{l}1,050 \\
1,370 \\
1,684 \\
1,953\end{array}$ & $\begin{array}{l}P \\
X \\
X \\
X\end{array}$ & $\mathrm{C}$ & $\mathrm{AM}$ & $\begin{array}{l}1988 \\
1989 \\
1990 \\
1991\end{array}$ & $\begin{array}{r}53.1 \\
114.4 \\
126.0 \\
109.9\end{array}$ & $\begin{array}{l}163 \\
351 \\
387 \\
337\end{array}$ & $\begin{array}{l}E \\
M \\
E \\
E\end{array}$ & $\begin{array}{r}14 \\
7 \\
31\end{array}$ \\
\hline \multicolumn{17}{|c|}{ AREA 25} \\
\hline J-11 WW & 364706116170601 & 364706 & 1161706 & $07-19-57$ & 3,443 & 1,327 & $\begin{array}{l}1,075 \\
1,242\end{array}$ & $\begin{array}{l}1,095 \\
1,298\end{array}$ & $\begin{array}{l}\mathrm{P} \\
\mathrm{P}\end{array}$ & $\mathrm{V}$ & AFFCR & $\begin{array}{l}1959 \\
1960\end{array}$ & $\begin{array}{l}12.1 \\
14.8\end{array}$ & $\begin{array}{l}37 \\
45\end{array}$ & $\begin{array}{l}M \\
M\end{array}$ & \\
\hline $\mathrm{J}-12 \mathrm{WW}^{3}$ & 364554116232400 & 364554 & 1162324 & $10-09-57$ & 3,128 & 887 & 793 & 868 & $\mathrm{P}$ & $\mathrm{V}$ & AFFCR & $\begin{array}{l}1961 \\
1962\end{array}$ & $\begin{array}{l}30.0 \\
60.8\end{array}$ & $\begin{array}{r}92 \\
187\end{array}$ & $\begin{array}{l}\mathrm{R} \\
\mathrm{R}\end{array}$ & \\
\hline$J-12 W W^{3}$ & 364554116232401 & 364554 & 1162324 & $08--68$ & 3,128 & 1,139 & $\begin{array}{l}793 \\
887\end{array}$ & $\begin{array}{r}868 \\
1,019\end{array}$ & $\begin{array}{l}\mathrm{P} \\
\mathrm{X}\end{array}$ & $\mathrm{V}$ & AFFCR & $\begin{array}{l}1981 \\
1982 \\
1983 \\
1984 \\
1985\end{array}$ & $\begin{array}{l}37.2 \\
18.7 \\
24.6 \\
24.8 \\
26.2\end{array}$ & $\begin{array}{r}114 \\
57 \\
75 \\
76 \\
80\end{array}$ & $\begin{array}{l}\mathrm{M} \\
\mathrm{M} \\
\mathrm{M} \\
\mathrm{E} \\
\mathrm{E}\end{array}$ & $\begin{array}{r}8 \\
20\end{array}$ \\
\hline & & & & & & & & & & & & $\begin{array}{l}1986 \\
1987 \\
1988 \\
1989 \\
1990 \\
1991\end{array}$ & $\begin{array}{l}28.6 \\
20.3 \\
20.5 \\
33.6 \\
31.2 \\
34.7\end{array}$ & $\begin{array}{r}88 \\
62 \\
63 \\
103 \\
96 \\
106\end{array}$ & $\begin{array}{l}\mathrm{M} \\
\mathrm{M} \\
\mathrm{M} \\
\mathrm{M} \\
\mathrm{M} \\
\mathrm{M}\end{array}$ & \\
\hline \multirow[t]{2}{*}{$J-13$ WW } & 364828116234001 & 364828 & 1162340 & $01-06-63$ & 3,318 & 3,488 & $\begin{array}{r}996 \\
1,301 \\
2,690 \\
3,385\end{array}$ & $\begin{array}{l}1,301 \\
1,386 \\
3,312 \\
3,488\end{array}$ & $\begin{array}{l}P \\
P \\
P \\
X\end{array}$ & $\mathrm{~V}$ & AFFCR & $\begin{array}{l}1983 \\
1984 \\
1985 \\
1986 \\
1987\end{array}$ & $\begin{array}{l}46.0 \\
41.1 \\
27.4 \\
17.4 \\
32.6\end{array}$ & $\begin{array}{r}141 \\
126 \\
84 \\
54 \\
100\end{array}$ & $\begin{array}{l}\mathrm{M} \\
\mathrm{E} \\
\mathrm{E} \\
\mathrm{M} \\
\mathrm{M}\end{array}$ & $\begin{array}{r}8 \\
20\end{array}$ \\
\hline & & & & & & & & & & & & $\begin{array}{l}1988 \\
1989 \\
1990 \\
1991\end{array}$ & $\begin{array}{l}25.5 \\
17.0 \\
20.5 \\
16.5\end{array}$ & $\begin{array}{l}78 \\
52 \\
63 \\
51\end{array}$ & $\begin{array}{l}M \\
E \\
E \\
M\end{array}$ & $\begin{array}{r}14 \\
7\end{array}$ \\
\hline
\end{tabular}

${ }^{1}$ Measurements not corrected for borehole deviation from vertical.

${ }^{2}$ Pumping ceased August 29, 1991.

${ }^{3}$ Repeated NTS hole numbers reflect more than one hole completion.

${ }_{5}^{4}$ New well, not currently in use.

${ }^{5}$ Intermittent use for cleaning purposes at the U.S. Environmental Protection Agency farm until the pump failed to start on October 7, 1991.

${ }^{6}$ Section of casing left in hole form 4,114 feet to 4,350 feet. 
Table 4. Tritium concentrations in water samples collected from test holes at the Nevada Test Site (NTS), Nye County, Nevada, water years 1990-91

NTS hole number--Drill-hole number assigned by Raytheon Services Nevada; see section "Site Designations" in text.

USGS standard identification--U.S. Geological Survey site designation; see section "Site Designations" in text.

Date hole completed--Date that borehole construction work ceased; see section "Measurements" in text.

Land-surface altitude--Datum is sea level. Value may not represent current altitude; see section "Depth to Water" in text.

Hole depth--Datum is land surface. Represents most recent accessible depth; see section "Measurements" in text.

Depth of open interval--Datum is land surface. Bottom of deepest open interval may be deeper than present accessible hole depth. Type: $P$, perforated or slotted casing; $S$, screen; $X$, open (uncased) hole.

Water sample--Water samples are analyzed by Environmental Monitoring Systems Laboratory of U.S. Environmental Protection Agency.

Tritium concentration--Analytical results and associated uncertainties are in $\mathrm{pCi} / \mathrm{L}$ (picocuries per liter). Laboratory analyses are reported as most probable value \pm 2 standard deviations counting error. Negative value of tritium concentration indicates that activity of sample was less than blank used in calibration procedures.

\begin{tabular}{|c|c|c|c|c|c|c|c|c|c|c|c|}
\hline \multirow{2}{*}{$\begin{array}{l}\text { NTS hole } \\
\text { number }\end{array}$} & \multirow{2}{*}{$\begin{array}{l}\text { USGS standard } \\
\text { Identification }\end{array}$} & \multirow{2}{*}{$\begin{array}{l}\text { Latitude } \\
\text { (degrees, } \\
\text { minutes, } \\
\text { seconds) }\end{array}$} & \multirow{2}{*}{$\begin{array}{l}\text { Longitude } \\
\text { (degrees, } \\
\text { minutes, } \\
\text { seconds) }\end{array}$} & \multirow{2}{*}{$\begin{array}{l}\text { Date hole } \\
\text { completed } \\
\text { (month, } \\
\text { day, year) }\end{array}$} & \multirow{2}{*}{$\begin{array}{l}\text { Land- } \\
\text { surface } \\
\text { altitude } \\
\text { (feet) }\end{array}$} & \multirow{2}{*}{$\begin{array}{l}\text { Hole } \\
\text { depth } \\
\text { (feet) }\end{array}$} & \multicolumn{3}{|c|}{$\begin{array}{c}\text { Depth of } \\
\text { open Interval }\end{array}$} & \multicolumn{2}{|c|}{ Water sample } \\
\hline & & & & & & & $\begin{array}{c}\text { Top } \\
\text { (feet) }\end{array}$ & $\begin{array}{l}\text { Bottom } \\
\text { (feet) }\end{array}$ & Type & $\begin{array}{c}\text { Date } \\
\text { (month, } \\
\text { day, year) }\end{array}$ & $\begin{array}{l}\text { Tritlum } \\
\text { (plcocuries } \\
\text { per liter) }\end{array}$ \\
\hline \multicolumn{12}{|c|}{ AREA 3} \\
\hline $\mathrm{U}-3 \mathrm{mt}$ & 370348116024301 & 370348 & 1160243 & $05-31-90$ & 4,067 & 1,545 & 110 & 1,550 & $\mathrm{X}$ & $06-18-91$ & $17 \pm 4$ \\
\hline UE-3e 4-2 & 370411116025911 & 370411 & 1160259 & $03-22-90$ & 4,082 & 1,919 & 1,887 & 1,908 & $S$ & $06-19-91$ & $104,000 \pm 754$ \\
\hline \multicolumn{12}{|c|}{ AREA 4} \\
\hline U-4av & 370547116041103 & 370547 & 1160411 & $08-18-90$ & 4,178 & 1,687 & $\begin{array}{l}117 \\
118 \\
119\end{array}$ & $\begin{array}{r}118 \\
119 \\
1,700\end{array}$ & $\begin{array}{l}\mathrm{X} \\
\mathrm{X} \\
\mathrm{X}\end{array}$ & $09-12-90$ & $6 \pm 6$ \\
\hline UE- $4 t^{2}$ & 370556116025404 & 370556 & 1160254 & $05-05-89$ & 4,144 & 1,721 & 1,619 & 1,721 & $\mathrm{X}$ & $08-01-90$ & $2 \pm 5$ \\
\hline UE-4t 1 & 370556116025405 & 370556 & 1160254 & $10-24-90$ & 4,144 & 2,010 & $\begin{array}{l}1,906 \\
1,963\end{array}$ & $\begin{array}{l}2,010 \\
1,992\end{array}$ & $\begin{array}{l}\mathrm{X} \\
\mathrm{P}\end{array}$ & $06-19-91$ & $224 \pm 6$ \\
\hline $\mathrm{UE}-4 \mathrm{t} 2$ & 370556116025406 & 370556 & 1160254 & $10-24-90$ & 4,144 & 1,754 & $\begin{array}{l}1,564 \\
1,664\end{array}$ & $\begin{array}{l}1,754 \\
1,724\end{array}$ & $\begin{array}{l}X \\
P\end{array}$ & $06-19-91$ & $26 \pm 4$ \\
\hline
\end{tabular}


Table 4. Tritium concentrations in water samples collected from test holes at the Nevada Test Site (NTS), Nye County, Nevada, water years 1990-91--Continued

\begin{tabular}{|c|c|c|c|c|c|c|c|c|c|c|c|}
\hline \multirow{2}{*}{$\begin{array}{c}\text { NTS hole } \\
\text { number }\end{array}$} & \multirow{2}{*}{$\begin{array}{l}\text { USGS standard } \\
\text { identification }\end{array}$} & \multirow{2}{*}{$\begin{array}{l}\text { Latitude } \\
\text { (degrees, } \\
\text { minutes, } \\
\text { seconds) }\end{array}$} & \multirow{2}{*}{$\begin{array}{l}\text { Longitude } \\
\text { (degrees, } \\
\text { minutes, } \\
\text { seconds) }\end{array}$} & \multirow{2}{*}{$\begin{array}{l}\text { Date hole } \\
\text { completed } \\
\text { (month, } \\
\text { day, year) }\end{array}$} & \multirow{2}{*}{$\begin{array}{l}\text { Land- } \\
\text { surface } \\
\text { altitude } \\
\text { (feet) }\end{array}$} & \multirow{2}{*}{$\begin{array}{l}\text { Hole } \\
\text { depth } \\
\text { (feet) }\end{array}$} & \multicolumn{3}{|c|}{$\begin{array}{c}\text { Depth of } \\
\text { open Interval }\end{array}$} & \multicolumn{2}{|c|}{ Water sample } \\
\hline & & & & & & & $\begin{array}{c}\text { Top } \\
\text { (feet) }\end{array}$ & $\begin{array}{c}\text { Bottom } \\
\text { (feet) }\end{array}$ & Type & $\begin{array}{c}\text { Date } \\
\text { (month, } \\
\text { day, year) }\end{array}$ & $\begin{array}{c}\text { Tritium } \\
\text { (picocuries } \\
\text { per liter) }\end{array}$ \\
\hline \multicolumn{12}{|c|}{ AREA 19} \\
\hline U-19bh & 371349116222001 & 371349 & 1162220 & $06-14-91$ & 6,768 & 2,148 & $\begin{array}{l}70 \\
72\end{array}$ & $\begin{array}{r}72 \\
2,148\end{array}$ & $\begin{array}{l}x \\
x\end{array}$ & $\begin{array}{l}07-02-91 \\
07-08-91 \\
07-26-91 \\
09-09-91\end{array}$ & $\begin{array}{r}4 \pm 4 \\
2 \pm 3 \\
256 \pm 7 \\
78 \pm 5\end{array}$ \\
\hline \multicolumn{12}{|c|}{ AREA 20} \\
\hline $\mathrm{U}-20 \mathrm{ax}$ & 371350116264701 & 371350 & 1162647 & $08-21-87$ & 6,536 & 2,200 & 62 & 2,200 & $\mathrm{X}$ & $09-10-91$ & $-1 \pm 3$ \\
\hline U-20bb 1 & 371452116293903 & 371452 & 1162939 & $05-07-90$ & 6,227 & 2,322 & $\begin{array}{l}60 \\
62\end{array}$ & $\begin{array}{r}62 \\
2,345\end{array}$ & $\begin{array}{l}X \\
X\end{array}$ & $05-15-90$ & $2 \pm 7$ \\
\hline $\mathrm{U}-20 \mathrm{bg}$ & 371414116242901 & 371414 & 1162429 & $12-19-90$ & 6,567 & 2,200 & $\begin{array}{r}58 \\
540\end{array}$ & $\begin{array}{r}540 \\
2,200\end{array}$ & $\begin{array}{l}X \\
X\end{array}$ & $09-10-91$ & $167 \pm 6$ \\
\hline
\end{tabular}

${ }^{1}$ Construction information not corrected for borehole deviation from vertical.

${ }^{2}$ Packers set at 1,619 and 1,721 feet. Sample reflects interval between packers. 